\title{
Cognitive Neurophysiology and in vivo Neurochemistry of Bipolar Disorder
}

Citation for published version (APA):

Atagün, M. I. (2020). Cognitive Neurophysiology and in vivo Neurochemistry of Bipolar Disorder. [Doctoral Thesis, Maastricht University]. Maastricht University. https://doi.org/10.26481/dis.20200527ma

Document status and date:

Published: 01/01/2020

DOI:

10.26481/dis.20200527ma

Document Version:

Publisher's PDF, also known as Version of record

\section{Please check the document version of this publication:}

- A submitted manuscript is the version of the article upon submission and before peer-review. There can be important differences between the submitted version and the official published version of record.

People interested in the research are advised to contact the author for the final version of the publication, or visit the DOI to the publisher's website.

- The final author version and the galley proof are versions of the publication after peer review.

- The final published version features the final layout of the paper including the volume, issue and page numbers.

Link to publication

\footnotetext{
General rights rights.

- You may freely distribute the URL identifying the publication in the public portal. please follow below link for the End User Agreement:

www.umlib.nl/taverne-license

Take down policy

If you believe that this document breaches copyright please contact us at:

repository@maastrichtuniversity.nl

providing details and we will investigate your claim.
}

Copyright and moral rights for the publications made accessible in the public portal are retained by the authors and/or other copyright owners and it is a condition of accessing publications that users recognise and abide by the legal requirements associated with these

- Users may download and print one copy of any publication from the public portal for the purpose of private study or research.

- You may not further distribute the material or use it for any profit-making activity or commercial gain

If the publication is distributed under the terms of Article $25 \mathrm{fa}$ of the Dutch Copyright Act, indicated by the "Taverne" license above, 


\section{Cognitive Neurophysiology and in vivo Neurochemistry of Bipolar Disorder}

Murat Ilhan Atagun MD 



\section{Cognitive Neurophysiology and in vivo Neurochemistry of Bipolar Disorder}

Murat Ilhan Atagun MD 


\title{
Cognitive Neurophysiology and in vivo Neurochemistry of Bipolar Disorder
}

\author{
DISSERTATION \\ to obtain the degree of Doctor at the Maastricht University, \\ on the authority of the Rector Magnificus, \\ Prof. Dr. Rianne M. Letschert \\ in accordance with the decision of the Board of Deans, \\ to be defended in public \\ on Wednesday $27^{\text {th }}$ of May 2020 , at $14: 00$ hours
}

by

Murat İlhan Atagün

Born in 22th April 1978, Elazığ, Turkey 
Supervisor:

Prof. Dr. T.A.M.J. van Amelsvoort

Co-supervisors:

Dr. S. Gülöksüz

Dr. M. Drukker

\section{Assessment Committee:}

Prof. Dr. D.E.J. Linden (chair)

Prof. Dr. R.W. Kupka

Dr. D.H. Nieman

Dr. C. Vingerhoets 
Dedicated to my Professors

Timuçin Oral, Ayşegül Özerdem, Erol Başar, Cüneyt Evren, Özcan Erel, Dost Öngür and many others 


\section{Paraynmphs}

Özlem Devrim Balaban

Zeliha Atagün 


\section{Contents}

CHAPTER 1 Introduction

CHAPTER 2 Decrease Of Theta Response In Euthymic Bipolar Patients During An Oddball Paradigm

CHAPTER 3 Decrease of Event-Related Delta Oscillations in Euthymic Patients with Bipolar Disorder

CHAPTER 4 Lithium Excessively Enhances Event Related Beta Oscillations in Patients with Bipolar Disorder

CHAPTER 5 Meta-analysis of Auditory P50 Sensory Gating in Schizophrenia and Bipolar Disorder

CHAPTER 6 Investigation of Heschl's gyrus and planum temporale in patients with schizophrenia and bipolar disorder: A proton magnetic resonance spectroscopy study

CHAPTER 7 Neurochemical Differences Between Bipolar Disorder Type I and II in Superior Temporal Cortices: A Proton Magnetic Resonance Spectroscopy Study

CHAPTER 8 Perisylvian GABA levels in schizophrenia and bipolar disorder

CHAPTER 9 Discussion

CHAPTER 10 Summary

CHAPTER 11 Valorization

Acknowledgement

Curriculum Vitae

List of Publications 


\section{CHAPTER 1}

Introduction 


\section{.}


Bipolar disorder (BD) is a mental disorder with an unpredictable relapsing-remitting course with manic or depressive episodes. Overall prevalence of BD subtypes is approximately $4.4 \%$ of the general population (Merikangas et al., 2007). BD is one of the most debilitating illnesses worldwide (Wittchen et al., 2011) and its disturbance is associated with recurrent mood disorder episodes, as well as neurocognitive dysfunction and psychiatric comorbidities (Grande et al., 2016; Malhi et al., 2007). Symptoms include changes in mood, energy, motor activity, sleep, appetite, thought, and cognition (AmericanPsychiatricAssociation, 2013). Cognitive symptoms are listed among the clinical symptoms in BD. Furthermore, neurocognitive impairment is a core dimension of $\mathrm{BD}$, which persists throughout euthymic episodes (Bora, 2018). Accordingly, attention, memory, and executive function domains are the most commonly disturbed cognitive functions in BD.

\section{In Vivo Assessment of Cognition}

Large numbers of neurons are distributed in the specialized areas of the brain for sophisticated networks for perception and cognition in humans. Coordination of these networks by oscillations is suggested to be provided by low (theta, alpha) and high (beta, gamma) frequency oscillations via the representation of neural correlates of sensory or cognitive information (Varela et al., 2001). The brain networks are at different levels and each cognitive function is associated with distinct neural networks (Petersen and Sporns, 2015). Neuroimaging techniques allow for the examination of individual networks associated with cognitive functions. The application of non-invasive imaging technologies has advanced the investigation of the neural basis of cognition through the mechanistic studies of neurocognitive circuits. Among the currently available dynamic functional assessment technologies, functional magnetic resonance imaging (fMRI), functional near infrared spectroscopy (fNIRS), and electroencephalography (EEG) are the most commonly used methods. While fMRI is able to characterize the spatial distribution of the networks, EEG offers unique information about the synchronization capabilities of the neural networks with high temporal resolution. In other words, despite its poor spatial resolution, temporal resolution is the major advantage of the EEG. This advantage makes the EEG a unique method for task-based cognition studies.

The scalp-EEG records the electrical activity of the brain, whereas the magnetic resonance imaging (MRI) is based on variations of magnetic vectors of water molecules. Radiofrequency pulses align and release molecular directions and the changes are captured and translated into images. The iron atoms add magnetic properties to the hemoglobin molecule and binding or 
releasing oxygen may alter its magnetization. Furthermore, functionally active brain regions induce vasomotor changes and increase blood supply in response to activation, which is called hemodynamic response. The hemodynamic changes are captured by the fMRI and functionally active brain regions are detected. The functional near infrared spectroscopy (fNIRS) is also able to capture changes in the hemodynamic response (Herold et al., 2018). Alterations in the infrared light illumination of the superficial cortical tissues depend on changes in their oxyhemoglobin content. This change is sensed and measured by the fNIRS (Herold et al., 2018). In addition, the MRI can detect biomolecules, because their molecular configuration possesses biophysical properties. Glutamate and glutamine, $\mathrm{N}$-acetylaspartate, choline, inositol, creatine or GABA can be detected by proton magnetic resonance spectroscopy (H-MRS), whereas phosphorous MRS (P-MRS) is able to measure bioenergetic molecules consisting of phosphorus atoms. Oxygen, carbon, lithium or fluorine MRS coils are also available for measuring specific molecules in vivo. However, the EEG signal is the direct response of the actively working neurons that contribute to the cognitive process.

Coherent brain functions might be achieved by synchronizing dedicated neuron groups that account for integrative brain functions. Neurons are organized to perform a particular brain function and resonate at a frequency, called oscillation. The term oscillation designates a repeated variation of a parameter (current or voltage) between two extreme values with an optional requirement of the regularity of the variation. Brain oscillations are grouped according to their frequencies: delta $(0.5-3.5 \mathrm{~Hz})$, theta $(4-8 \mathrm{~Hz})$, alpha $(8-13 \mathrm{~Hz})$, beta $(14-28 \mathrm{~Hz})$ and gamma (30-70 Hz) rhythms (Başar, 1999). Each frequency is associated with specific brain functions. Theories of functional significance for these brain rhythms are conclusive and supported by evidence. Oscillations may occur either spontaneously, or induced or evoked by a sensory stimulus, which might be event-related (Başar, 1998). A stimulus evokes a coordinated response in the neuron population and the brain transits from irregular neural activity to synchronous network-level work. Although complex connections establish sophisticated networks in the brain, dedicated networks are assigned for specific functions. The type of the function determines the brain region and the neuronal assembly produces a specific oscillation at a particular frequency and phase. Synchronous firing of the neurons is provided by integrative brain functions to set up networks. Psychiatric disorders might be associated with pathologies that disrupt these integrative brain functions and might be expressed in the dysfunction of certain networks. 
Cognitive function can be examined through studying the EEG evaluation and response processes instigated by specific tasks that artificially produce an environmental stimulus. The task may be designed to provoke either a cognitive (top-down operation) or an automatic (bottom-up) processing. Bottom-up (stimulus-driven) tasks (i.e. mismatch negativity [MMN], auditory steady-state response (ASSR) or dual-click paradigm [P50]) are independent of performance and available for all clinical populations. In contrast, top-down tasks rely on mental operations on sensory or cognitive information. Top-down tasks are associated with attention, decision making, and working memory and these functions are performed by complex networks consisting of prefrontal, parietal and temporal cortices (Wagner et al., 1998). Each stimulus of the cognitive tasks induces changes in specific brain waves, either in their magnitudes or phases. Moreover, maintenance of specific frequency bands across trials with respect to an event indicates consistent and quantifiable brain activity or response. In short, with its high temporal resolution, the EEG may facilitate assessment of cognitive networks by means of cognitive tasks (Kenemans and Kahkonen, 2011).

\section{Brain Oscillations in Bipolar Disorder}

The classical event-related potentials analysis revealed that the classical P300 magnitude might either be decreased (Fridberg et al., 2009; O'Donnell et al., 2004b; Salisbury et al., 1998) or remain unchanged in bipolar disorder compared to healthy controls (Hall et al., 2007a; Kaya et al., 2007; Schulze et al., 2008). On the other hand, P200 and N200 are diminished in BD compared to schizophrenia and healthy control groups (Ethridge et al., 2015; Hamm et al., 2013; Mokhtari et al., 2016). Resting-state EEG may exhibit neurophysiological activity of the default mode network. Patients with the diagnosis of BD had increased magnitudes in delta, theta and alpha activity at rest (Narayanan et al., 2014). A high-resolution EEG source localization (LORETA) study reported that patients with BD either in manic or depressive states showed decreased theta and enhanced beta power in prefrontal and parietal cortices and temporal pole in resting state condition (Painold et al., 2014). On the other hand, the most consistent finding was the gamma band power reduction in euthymia (Oda et al., 2012; Parker et al., 2019; Rass et al., 2010) and mania (O'Donnell et al., 2004a) upon Auditory Steady State Response (ASSR) task. Gamma (Lee et al., 2010) and beta band (Parker et al., 2019) are also disturbed in BD. The ASSR and mismatch negativity (MMN) tasks enable the examination of the auditory cortices and their connected networks (Garrido et al., 2009; Sivarao, 2015). Thus, alterations in schizophrenia and BD may point to the pathology of the auditory cortices. 
Psychosis was the most important clinical determinant of the EEG findings in patients with BD (Hamm et al., 2013; Lundin et al., 2018). However, almost all psychotropic medications alter the neurophysiology; therefore, EEG studies with medicated patients might be biased to a degree. Clinical EEG studies in patients with BD showed inconsistent results because of confounders, of which the most pronounced were cognitive and clinical heterogeneity in patients with BD (Bora et al., 2019) and psychotropic treatments (Atagun, 2016).

\section{In Vivo Assessment of Neurochemistry (magnetic resonance spectroscopy) in Bipolar Disorder}

Proton magnetic resonance imaging spectroscopy (H-MRS) is able to identify molecules with their physical configuration in a given volume. The point-resolved spectroscopy sequence (PRESS) provides quantitation of glutamate, $\mathrm{N}$-acetylaspartate, choline, inositol and creatine, whereas Meshcher-Garwood point-resolved spectroscopy sequence (MEGAPRESS) is able to quantitate the GABA neurotransmitter (Mullins et al., 2014). The standard sizes of the voxels of the MRS are $8 \mathrm{cc}(2 \mathrm{X} 2 \mathrm{X} 2 \mathrm{~cm})$ in the PRESS sequence and $27 \mathrm{cc}(3 \times 3 \times 3 \mathrm{~cm})$ in the MEGAPRESS sequence.

The MRS signal consists of different peaks and resonances. Each peak is related with a specific type of compound or combination of metabolites. Glutamate is one of the most abundant aminoacids in the brain. Because it lacks methyl groups and has methylene and methine groups, glutamate is measured in three different peaks by the proton-MRS $\left({ }^{1} \mathrm{H}-\mathrm{MRS}\right)(2.08,2.34$ and $3.74 \mathrm{ppm})$. These signals from glutamate are difficult to distinguish from the analogous peaks arising from glutamine at about 2.44, 2.12, and 3.75ppm (Maddock and Buonocore, 2012). Glutamine to glutamate ratio in concentration is $40 / 60$ percent (Jensen et al. 2009) and signals of glutamine confound glutamate peaks unless optimized MRS methods are used. GABA and glutathione are also suspected to contribute to these signals. Therefore, this combined signal is abbreviated as glutamix ( $\mathrm{glx})$ and this resonance is considered an indicator of glutamatergic neurotransmission (Maddock and Buonocore, 2012). The N-acetylaspartate (NAA) resonance (at 2.02ppm) contains largely NAA and a small proportion is $\mathrm{N}$-acetylaspartylglutamate (NAAG). Acetyl coenzyme A and aspartate are the components of NAA and it is synthesized in mitochondria (Moore and Galloway, 2002). Thus, NAA is an acetyl donor for acetyl coenzyme A. NAA is also known to cycle between neurons and oligodendrocytes, which contain the enzyme that catabolize NAA-aspartoacetylase. The cycle of the NAAG is similar to the NAA, but its catabolic enzyme (NAAG peptidase) is located on the cell membrane of 
astrocytes (Baslow, 2000). Choline peak (3.23ppm) is composed of phosphorylcholine (PC) and glycerophosphorylcholine (GPC); therefore, it is thought to be a potential biomarker for membrane phospholipid metabolism (Moore and Galloway, 2002). Choline compounds embedded in the membranes are not measurable by the MRS, but free phosphatidylcholine is visible to it. The choline peak is higher in white matter, because it has a larger amount of choline related compounds. During the turnover of the membrane, the free proportion of the choline is measured; therefore, neurodegenerative diseases that increase phospholipid turn-over may amplify this peak. The myo-inositol (mI) peak $(3.56 \mathrm{ppm})$ consists of $\mathrm{mI}$ and minor contributions from glycine and inositol-1-phosphate (Moore and Galloway, 2002). The phosphoinositol messenger pathway, the metabolism of membrane phospholipids, and the regulation of neuronal osmolarity are physiological functions of $\mathrm{mI}$. The creatine $(\mathrm{Cr})$ peak (3.02 ppm) consists of phosphocreatine and creatine combined and these metabolites are high energy phosphate compounds that can easily diffuse through membranes (Maddock and Buonocore, 2012). Cr levels are related with the global health of brain cells and alterations may indicate impairment of their function or integrity.

It has been consistently found in MRS studies that glutamatergic compound levels are increased in patients with bipolar disorder (Gigante et al., 2012; Yuksel and Ongur, 2010) and schizophrenia (Merritt et al., 2016). Levels of other metabolites including GABA are less consistent and variable across brain regions (Egerton et al., 2017; Yildiz-Yesiloglu and Ankerst, 2006). Some studies reported decreased levels of metabolites, whereas some studies reported no change. In addition to clinical heterogeneity, most of the studies are cross-sectional and performed with small sample sizes, and voxels were located in different brain regions. However, studies have reported relationship between mismatch negativity amplitude and glutamatergic compound levels in the hippocampus (Chitty et al., 2015; Kaur et al., 2019). In addition, P300 amplitude was related to glutamatergic compound levels in the anterior ciungulate cortex in another study (Hall et al., 2015b). These findings indicate that glutamatergic compounds might be related with the disorder and its related mechanisms.

The aim of this thesis is to present a neurophysiological and neurochemical outline to alterations in cognitive function in bipolar disorder. Auditory oddball paradigm and P50 sensory gating (SG) are based on auditory networks and sensory systems provide bases for cognitive function. The auditory cortices are vulnerability zones for psychosis (Vita et al., 2012) and bipolar disorder (Oribe et al., 2010). Therefore, my aim was to examine the neurophysiological 
assessment of auditory task-based (oddball and P50 SG paradigms) EEG studies and neurochemistry of auditory cortices.

Theta frequency oscillations are suggested to be involved with the integration of distant brain regions (von Stein and Sarnthein, 2000). Furthermore, cortical theta rhythm reflects dynamic interactions between the hippocampal system and the neocortex during cognitive tasks, particularly memory function (Bastiaansen and Hagoort, 2003). Thus, scalp-EEG theta oscillation provides relevant information about memory related processes in the networks associated with the hippocampal formation. Indeed, theta rhythmic activity may facilitate the formation of dynamic neural assemblies that constitute the neural processors of cognition. According to the networks approach, etiopathogenetic mechanism of BD is related with the disruption in the corticolimbic and fronto-temporal networks (Benes and Berretta, 2001; Blumberg et al., 2002; Phillips et al., 2003). These networks also serve cognitive and emotion regulation functions (Strakowski et al., 2005). Therefore, cognitive task-based theta oscillations might be influenced by the pathogenesis of $\mathrm{BD}$ and altered theta responses are likely to represent neurophysiologic correlates of cognitive deficits in BD (Sakowitz et al., 2000). In Chapter 2, alteration in event-related theta oscillations in medication-free patients with bipolar disorder is described (Atagun et al., 2013). The oddball paradigm was the cognitive task of the study. The oddball paradigm is a valid working memory task that is relevant for the assessment of neurophysiological correlates of cognitive dysfunction in BD.

Delta waves in the frequency range of 1-4 Hz are usually observed during the slow-wave sleep, indicating hypersynchrony in the neuronal activities of the nuclei. Delta oscillations are suggested to be related with different frequency oscillations, rather than being a specific independent oscillation (IFSECN) (1974). On the other hand, interaction within thalamocortical networks yields a complex pattern whose reflection at the EEG level takes the shape of polymorphic waves, including delta oscillations (McCormick and Pape, 1990). Delta oscillations may also be generated by cortical neurons, probably with unique cellular mechanisms and they join the neurocognitive processes (Guntekin and Basar, 2016). However, event-related delta oscillations have been rarely studied in the general population or in psychiatric populations. On the other hand, delta frequency oscillations are believed to be the major component of the P300 amplitude and thus might be disturbed in BD. Chapter 3 describes the assessment of event-related delta oscillations in medication-free patients with BD (Atagun et al., 2014). 
Because of their large spectral interval and functional difference, beta oscillations are classically divided into two sub-bands, B-1 in 14 to $20 \mathrm{~Hz}$ and B-2 in 20 to $30 \mathrm{~Hz}$ intervals in the EEG literature. A well-established functional role of beta oscillations is the programming of voluntary motor movements. Although a variety of beta-oscillations with different spatial distribution and reactivity exists, the majority of the beta-oscillations are outputs of pyramidalneurons of deep layers (layer $\mathrm{V}$ mostly). Interestingly, beta-oscillations may have a nonsynaptic origin, in which electrical coupling emerges by axonal excitation in the layer $\mathrm{V}$ of the cortex (Roopun et al., 2006). Beta-2 rhythm in this network is set by cholinergic currents, while NMDA receptor antagonist ketamine also increases the ability of the prefrontal cortex to generate beta oscillations. In addition, because benzodiazepines increase the power of beta oscillations and decrease their frequency, it is proposed that a cortical inter-neuronal network might be responsible for the generation of cortical beta rhythms (Roopun et al., 2006; Traub et al., 2004). Neuroplastic changes in the brain drives networks to oscillate at beta frequency instead of gamma frequency (Whittington et al., 2000). These changes indicate that pharmacological interventions may induce changes in beta oscillations (Yoshimura et al., 2007). Because of its pharmacological actions and biophysical properties, lithium may cause alterations in neurophysiology. As lithium is the most prescribed mood stabilizer in BD and its mechanisms of action remain unclear, in Chapter 4, I investigated the effects of lithium on brain oscillations in patients with BD (Atagun et al., 2015a). Patients with bipolar disorder, both on and off lithium monotherapy were compared with a healthy control group.

Sensory gating is the mechanism that provides filters to reduce the perceived environmental stimuli and the dual-click paradigm is a classical measure of sensory gating in EEG experiments (Baker et al., 1987). Two successive clicks are presented and their responses are compared. Response to the second click should be lower than the first click. The difference between the amplitudes is believed to be related with the cognitive process of sensory gating. Alteration in this early attentive information processing decreases brain's ability to filter redundant inputs and overloads higher brain functions, leading to cognitive fragmentation and behavioral disturbances (Venables, 1964). Sensory gating has been widely assessed in both schizophrenia (Bramon et al., 2004; de Wilde et al., 2007b) and BD (Cheng et al., 2016). Furthermore, it has been documented with magnetoencephalography (Thoma et al., 2005; Wang et al., 2014a; Wang et al., 2014b) and MRI (Thoma et al., 2004) that P50 SG alterations are related with superior temporal lobes in bipolar disorder and schizophrenia. On the other hand, little is known about SG alterations in the different terms of the disorders and the effects of medications. 
Chapter 5 presents the meta-analysis of P50 studies in bipolar disorder and schizophrenia (Atagun et al. Submitted). Clinical states and effects of medications are assessed in this metaanalysis.

\section{In Vivo Neurochemistry of Superior Temporal Lobes in Patients with Bipolar Disorder}

The auditory cortices are located in the Heschl's gyrus and planum temporale in superior temporal lobes. Primary auditory cortices are surrounded by the Wernicke's Area and other association cortices are located around the belt between the temporal cortex and parietal cortex. These connected adjacent structures process auditory information and contact higher cognitive networks for further information processing. Since auditory cortices are shown to be functionally disturbed in the neuroimaging studies of BD and schizophrenia, they warrant further research. I aimed to assess the biochemistry of auditory cortices in schizophrenia and BD, by means of H-MRS. First, I investigated glutamate levels in patients with schizophrenia and type I BD in Chapter 6 (Atagun et al., 2015b) and type II in Chapter 7 (Atagun et al., 2017). Second, I investigated GABA levels in patients with schizophrenia and BD in Chapter 8 (Atagun et al., 2018).

The relationship between neurocognitive dysfunction and cortical alterations (in particular auditory cortices) in BD will be discussed. Clinical variations and potential confounders are going to be evaluated. Neurophysiology is a good way to evaluate cognitive function, since it provides the opportunity to assess dynamic changes through event-related experiments. Eventrelated spectral perturbations and phase synchronization might be accurate only with neuronal integrity and pathologies may disturb these mechanisms. Disturbed neuronal integrity might be related with structural as well as functional abnormalities, including chemical alterations. In Chapter 9, a comprehensive discussion will be presented.

\section{References}

1974. A glossary of terms most commonly used

by clinical electroencephalographers.
Electroencephalogr Clin Neurophysiol 37 (5), 538-548. 
AmericanPsychiatricAssociation,

2013.

Diagnostic and statistical manual of mental disorders. American Psychiatric Association, Arlington, VA, USA.

Atagun, M.I., 2016. Brain oscillations in bipolar disorder and lithium-induced changes. Neuropsychiatr Dis Treat 12, 589-601.

Atagun, M.I., Guntekin, B., Masali, B., Tulay, E., Basar, E., 2014. Decrease of eventrelated delta oscillations in euthymic patients with bipolar disorder. Psychiatry Res 223 (1), 43-48.

Atagun, M.I., Guntekin, B., Ozerdem, A., Tulay, E., Basar, E., 2013. Decrease of theta response in euthymic bipolar patients during an oddball paradigm. Cogn Neurodyn 7 (3), 213-223.

Atagun, M.I., Guntekin, B., Tan, D., Tulay, E.E., Basar, E., 2015a. Lithium excessively enhances event related beta oscillations in patients with bipolar disorder. J Affect Disord 170, 59-65.

Atagun, M.I., Sikoglu, E.M., Can, S.S., Karakas-Ugurlu, G., Ulusoy-Kaymak, S., Caykoylu, A., Algin, O., Phillips, M.L., Moore, C.M., Ongur, D., 2015 b. Investigation of Heschl's gyrus and planum temporale in patients with schizophrenia and bipolar disorder: a proton magnetic resonance spectroscopy study. Schizophr Res 161 (2-3), 202-209.

Atagun, M.I., Sikoglu, E.M., Can, S.S., Ugurlu, G.K., Kaymak, S.U., Caykoylu, A., Algin, O., Phillips, M.L., Moore, C.M., Ongur, D., 2018. Neurochemical differences between bipolar disorder type I and II in superior temporal cortices: A proton magnetic resonance spectroscopy study. J Affect Disord 235, 15-19.
Atagun, M.I., Sikoglu, E.M., Soykan, C., Serdar Suleyman, C., Ulusoy-Kaymak, S., Caykoylu, A., Algin, O., Phillips, M.L., Ongur, D., Moore, C.M., 2017. Perisylvian GABA levels in schizophrenia and bipolar disorder. Neurosci Lett 637, 70-74.

Baker, N., Adler, L.E., Franks, R.D., Waldo, M., Berry, S., Nagamoto, H., Muckle, A., Freedman, R., 1987. Neurophysiological assessment of sensory gating in psychiatric inpatients: comparison between schizophrenia and other diagnoses. Biol Psychiatry 22 (5), 603-617.

Başar, E., 1998. Brain oscillations I: principles and approaches. Springer, Heidelberg, Germany.

Başar, E., 1999. Brain function and oscillations: II. Integrative brain function. Neurophysiology and cognitive processes. Springer, Heidelberg, Germany.

Baslow, M.H., 2000. Functions of N-acetyl-Laspartate and $\mathrm{N}$-acetyl-L-aspartylglutamate in the vertebrate brain: role in glial cellspecific signaling. J Neurochem 75 (2), 453459.

Bastiaansen, M., Hagoort, P., 2003. Eventinduced theta responses as a window on the dynamics of memory. Cortex 39 (4-5), 967992.

Benes, F.M., Berretta, S., 2001. GABAergic interneurons: implications for understanding schizophrenia and bipolar disorder. Neuropsychopharmacology 25 (1), 1-27.

Blumberg, H.P., Charney, D.S., Krystal, J.H., 2002. Frontotemporal neural systems in bipolar disorder. Semin Clin Neuropsychiatry 7 (4), 243-254.

Bora, E., 2018. Neurocognitive features in clinical subgroups of bipolar disorder: A meta-analysis. J Affect Disord 229, 125-134. 
Bora, E., Can, G., Ildiz, A., Ulas, G., Ongun, C.H., Inal, N.E., Ozerdem, A., 2019. Neurocognitive heterogeneity in young offspring of patients with bipolar disorder: The effect of putative clinical stages. J Affect Disord 257, 130-135.

Bramon, E., Rabe-Hesketh, S., Sham, P., Murray, R.M., Frangou, S., 2004. Metaanalysis of the P300 and P50 waveforms in schizophrenia. Schizophr Res 70 (2-3), 315329.

Cheng, C.H., Chan, P.S., Liu, C.Y., Hsu, S.C., 2016. Auditory sensory gating in patients with bipolar disorders: A meta-analysis. J Affect Disord 203, 199-203.

Chitty, K.M., Lagopoulos, J., Hickie, I.B., Hermens, D.F., 2015. Hippocampal glutamatergic/NMDA receptor functioning in bipolar disorder: A study combining mismatch negativity and proton magnetic resonance spectroscopy. Psychiatry Res 233 (2), 88-94.

de Wilde, O.M., Bour, L.J., Dingemans, P.M., Koelman, J.H., Linszen, D.H., 2007. A meta-analysis of P50 studies in patients with schizophrenia and relatives: differences in methodology between research groups. Schizophr Res 97 (1-3), 137-151.

Egerton, A., Modinos, G., Ferrera, D., McGuire, P., 2017. Neuroimaging studies of GABA in schizophrenia: a systematic review with meta-analysis. Transl Psychiatry 7 (6), e1147.

Ethridge, L.E., Hamm, J.P., Pearlson, G.D., Tamminga, C.A., Sweeney, J.A., Keshavan, M.S., Clementz, B.A., 2015. Event-related potential and time-frequency endophenotypes for schizophrenia and psychotic bipolar disorder. Biol Psychiatry 77 (2), 127-136.

Fridberg, D.J., Hetrick, W.P., Brenner, C.A., Shekhar, A., Steffen, A.N., Malloy, F.W.,
O'Donnell, B.F., 2009. Relationships between auditory event-related potentials and mood state, medication, and comorbid psychiatric illness in patients with bipolar disorder. Bipolar Disord 11 (8), 857-866.

Garrido, M.I., Kilner, J.M., Stephan, K.E., Friston, K.J., 2009. The mismatch negativity: a review of underlying mechanisms. Clin Neurophysiol 120 (3), 453-463.

Gigante, A.D., Bond, D.J., Lafer, B., Lam, R.W., Young, L.T., Yatham, L.N., 2012. Brain glutamate levels measured by magnetic resonance spectroscopy in patients with bipolar disorder: a meta-analysis. Bipolar Disord 14 (5), 478-487.

Grande, I., Berk, M., Birmaher, B., Vieta, E., 2016. Bipolar disorder. Lancet 387 (10027), 1561-1572.

Guntekin, B., Basar, E., 2016. Review of evoked and event-related delta responses in the human brain. Int J Psychophysiol 103, 43-52.

Hall, M.H., Jensen, J.E., Du, F., Smoller, J.W., O'Connor, L., Spencer, K.M., Ongur, D., 2015. Frontal P3 event-related potential is related to brain glutamine/glutamate ratio measured in vivo. Neuroimage 111, 186191.

Hall, M.H., Rijsdijk, F., Kalidindi, S., Schulze, K., Kravariti, E., Kane, F., Sham, P., Bramon, E., Murray, R.M., 2007. Genetic overlap between bipolar illness and eventrelated potentials. Psychol Med 37 (5), 667678.

Hamm, J.P., Ethridge, L.E., Shapiro, J.R., Pearlson, G.D., Tamminga, C.A., Sweeney, J.A., Keshavan, M.S., Thaker, G.K., Clementz, B.A., 2013. Family history of psychosis moderates early auditory cortical response abnormalities in non-psychotic 
bipolar disorder. Bipolar Disord 15 (7), 774786.

Herold, F., Wiegel, P., Scholkmann, F., Muller, N.G., 2018. Applications of Functional Near-Infrared Spectroscopy (fNIRS) Neuroimaging in Exercise(-)Cognition Science: A Systematic, MethodologyFocused Review. J Clin Med 7 (12).

Kaur, M., Chitty, K.M., Lagopoulos, J., Hickie, I.B., Duffy, S.L., Hermens, D.F., 2019. Elucidating the glutamatergic processes underlying mismatch negativity deficits in early stage bipolar disorder and schizophrenia: A combined (1)H-MRS and EEG study. J Psychiatr Res 113, 83-89.

Kaya, E., Aydemir, O., Selcuki, D., 2007. Residual symptoms in bipolar disorder: the effect of the last episode after remission. Prog Neuropsychopharmacol Biol Psychiatry 31 (7), 1387-1392.

Kenemans, J.L., Kahkonen, S., 2011. How human electrophysiology informs psychopharmacology: from bottom-up driven processing to top-down control. Neuropsychopharmacology 36 (1), 26-51.

Lee, P.S., Chen, Y.S., Hsieh, J.C., Su, T.P., Chen, L.F., 2010. Distinct neuronal oscillatory responses between patients with bipolar and unipolar disorders: a magnetoencephalographic study. J Affect Disord 123 (1-3), 270-275.

Lundin, N.B., Bartolomeo, L.A., O'Donnell, B.F., Hetrick, W.P., 2018. Reduced electroencephalogram responses to standard and target auditory stimuli in bipolar disorder and the impact of psychotic features: Analysis of event-related potentials, spectral power, and inter-trial coherence. Bipolar Disord 20 (1), 49-59.

Maddock, R.J., Buonocore, M.H., 2012. MR spectroscopic studies of the brain in psychiatric disorders. Curr Top Behav Neurosci 11, 199-251.

Malhi, G.S., Ivanovski, B., Hadzi-Pavlovic, D., Mitchell, P.B., Vieta, E., Sachdev, P., 2007. Neuropsychological deficits and functional impairment in bipolar depression, hypomania and euthymia. Bipolar Disord 9 (1-2), 114-125.

McCormick, D.A., Pape, H.C., 1990. Properties of a hyperpolarization-activated cation current and its role in rhythmic oscillation in thalamic relay neurones. J Physiol 431, 291318.

Merikangas, K.R., Akiskal, H.S., Angst, J., Greenberg, P.E., Hirschfeld, R.M., Petukhova, M., Kessler, R.C., 2007. Lifetime and 12-month prevalence of bipolar spectrum disorder in the National Comorbidity Survey replication. Arch Gen Psychiatry 64 (5), 543-552.

Merritt, K., Egerton, A., Kempton, M.J., Taylor, M.J., McGuire, P.K., 2016. Nature of Glutamate Alterations in Schizophrenia: A Meta-analysis of Proton Magnetic Resonance Spectroscopy Studies. JAMA Psychiatry 73 (7), 665-674.

Mokhtari, M., Narayanan, B., Hamm, J.P., Soh, P., Calhoun, V.D., Ruano, G., Kocherla, M., Windemuth, A., Clementz, B.A., Tamminga, C.A., Sweeney, J.A., Keshavan, M.S., Pearlson, G.D., 2016. Multivariate Genetic Correlates of the Auditory Paired Stimuli-Based P2 Event-Related Potential in the Psychosis Dimension From the BSNIP Study. Schizophr Bull 42 (3), 851-862.

Moore, G.J., Galloway, M.P., 2002. Magnetic resonance spectroscopy: neurochemistry and treatment effects in affective disorders. Psychopharmacol Bull 36 (2), 5-23.

Mullins, P.G., McGonigle, D.J., O'Gorman, R.L., Puts, N.A., Vidyasagar, R., Evans, C.J., Cardiff Symposium on, M.R.S.o.G., 
Edden, R.A., 2014. Current practice in the use of MEGA-PRESS spectroscopy for the detection of GABA. Neuroimage 86, 43-52.

Narayanan, B., O'Neil, K., Berwise, C., Stevens, M.C., Calhoun, V.D., Clementz, B.A., Tamminga, C.A., Sweeney, J.A., Keshavan, M.S., Pearlson, G.D., 2014. Resting state electroencephalogram oscillatory abnormalities in schizophrenia and psychotic bipolar patients and their relatives from the bipolar and schizophrenia network on intermediate phenotypes study. Biol Psychiatry. 76 (6), 456-465.

O'Donnell, B.F., Hetrick, W.P., Vohs, J.L., Krishnan, G.P., Carroll, C.A., Shekhar, A., 2004a. Neural synchronization deficits to auditory stimulation in bipolar disorder. Neuroreport. 15 (8), 1369-1372.

O'Donnell, B.F., Vohs, J.L., Hetrick, W.P., Carroll, C.A., Shekhar, A., 2004b. Auditory event-related potential abnormalities in bipolar disorder and schizophrenia. Int $\mathbf{J}$ Psychophysiol. 53 (1), 45-55.

Oda, Y., Onitsuka, T., Tsuchimoto, R., Hirano, S., Oribe, N., Ueno, T., Hirano, Y., Nakamura, I., Miura, T., Kanba, S., 2012. Gamma band neural synchronization deficits for auditory steady state responses in bipolar disorder patients. PLoS One 7 (7), e39955.

Oribe, N., Onitsuka, T., Hirano, S., Hirano, Y., Maekawa, T., Obayashi, C., Ueno, T., Kasai, K., Kanba, S., 2010. Differentiation between bipolar disorder and schizophrenia revealed by neural oscillation to speech sounds: an MEG study. Bipolar Disord. 12 (8), 804812.

Painold, A., Faber, P.L., Milz, P., Reininghaus, E.Z., Holl, A.K., Letmaier, M., PascualMarqui, R.D., Reininghaus, B., Kapfhammer, H.P., Lehmann, D., 2014. Brain electrical source imaging in manic and depressive episodes of bipolar disorder. Bipolar Disord. 16 (7), 690-702.
Parker, D.A., Hamm, J.P., McDowell, J.E., Keedy, S.K., Gershon, E.S., Ivleva, E.I., Pearlson, G.D., Keshavan, M.S., Tamminga, C.A., Sweeney, J.A., Clementz, B.A., 2019. Auditory steady-state EEG response across the schizo-bipolar spectrum. Schizophr Res. 209, 218-226.

Petersen, S.E., Sporns, O., 2015. Brain Networks and Cognitive Architectures. Neuron 88 (1), 207-219.

Phillips, M.L., Drevets, W.C., Rauch, S.L., Lane, R., 2003. Neurobiology of emotion perception I: The neural basis of normal emotion perception. Biol Psychiatry. 54 (5), 504-514.

Rass, O., Krishnan, G., Brenner, C.A., Hetrick, W.P., Merrill, C.C., Shekhar, A., O'Donnell, B.F., 2010. Auditory steady state response in bipolar disorder: relation to clinical state, cognitive performance, medication status, and substance disorders. Bipolar Disord. 12 (8), 793-803.

Roopun, A.K., Middleton, S.J., Cunningham, M.O., LeBeau, F.E., Bibbig, A., Whittington, M.A., Traub, R.D., 2006. A beta2-frequency $(20-30 \mathrm{~Hz})$ oscillation in nonsynaptic networks of somatosensory cortex. Proc Natl Acad Sci U S A. 103 (42), 15646-15650.

Sakowitz, O.W., Schurmann, M., Basar, E., 2000. Oscillatory frontal theta responses are increased upon bisensory stimulation. Clin Neurophysiol. 111 (5), 884-893.

Salisbury, D.F., Shenton, M.E., Sherwood, A.R., Fischer, I.A., Yurgelun-Todd, D.A., Tohen, M., McCarley, R.W., 1998. Firstepisode schizophrenic psychosis differs from first-episode affective psychosis and controls in P300 amplitude over left temporal lobe. Arch Gen Psychiatry. 55 (2), 173-180. 
Schulze, K.K., Hall, M.H., McDonald, C., Marshall, N., Walshe, M., Murray, R.M., Bramon, E., 2008. Auditory P300 in patients with bipolar disorder and their unaffected relatives. Bipolar Disord. 10 (3), 377-386.

Sivarao, D.V., 2015. The $40-\mathrm{Hz}$ auditory steady-state response: a selective biomarker for cortical NMDA function. Ann N Y Acad Sci. 1344, 27-36.

Strakowski, S.M., Delbello, M.P., Adler, C.M., 2005. The functional neuroanatomy of bipolar disorder: a review of neuroimaging findings. Mol Psychiatry. 10 (1), 105-116.

Thoma, R.J., Hanlon, F.M., Moses, S.N., Ricker, D., Huang, M., Edgar, C., Irwin, J., Torres, F., Weisend, M.P., Adler, L.E., Miller, G.A., Canive, J.M., 2005. M50 sensory gating predicts negative symptoms in schizophrenia. Schizophr Res. 73 (2-3), 311-318.

Thoma, R.J., Hanlon, F.M., Sanchez, N., Weisend, M.P., Huang, M., Jones, A., Miller, G.A., Canive, J.M., 2004. Auditory sensory gating deficit and cortical thickness in schizophrenia. Neurol Clin Neurophysiol. 2004, 62 .

Traub, R.D., Bibbig, A., LeBeau, F.E., Buhl, E.H., Whittington, M.A., 2004. Cellular mechanisms of neuronal population oscillations in the hippocampus in vitro. Annu Rev Neurosci. 27, 247-278.

Varela, F., Lachaux, J.P., Rodriguez, E., Martinerie, J., 2001. The brainweb: phase synchronization and large-scale integration. Nat Rev Neurosci. 2 (4), 229-239.

Venables, P.H., 1964. Input Dysfunction in Schizophrenia. Prog Exp Pers Res. 72, 1-47.

Vita, A., De Peri, L., Deste, G., Sacchetti, E., 2012. Progressive loss of cortical gray matter in schizophrenia: a meta-analysis and meta-regression of longitudinal MRI studies. Transl Psychiatry. 2, e190.

von Stein, A., Sarnthein, J., 2000. Different frequencies for different scales of cortical integration: from local gamma to long range alpha/theta synchronization. Int $\mathbf{J}$ Psychophysiol. 38 (3), 301-313.

Wagner, A.D., Schacter, D.L., Rotte, M., Koutstaal, W., Maril, A., Dale, A.M., Rosen, B.R., Buckner, R.L., 1998. Building memories: remembering and forgetting of verbal experiences as predicted by brain activity. Science 281 (5380), 1188-1191.

Wang, Y., Feng, Y., Jia, Y., Wang, W., Xie, Y., Guan, Y., Zhong, S., Zhu, D., Huang, L., 2014a. Auditory M50 and M100 sensory gating deficits in bipolar disorder: a MEG study. J Affect Disord. 152-154, 131-138.

Wang, Y., Jia, Y., Feng, Y., Zhong, S., Xie, Y., Wang, W., Guan, Y., Zhu, D., Huang, L., 2014b. Overlapping auditory M100 and M200 abnormalities in schizophrenia and bipolar disorder: a MEG study. Schizophr Res. 160 (1-3), 201-207.

Whittington, M.A., Faulkner, H.J., Doheny, H.C., Traub, R.D., 2000. Neuronal fast oscillations as a target site for psychoactive drugs. Pharmacol Ther. 86 (2), 171-190.

Wittchen, H.U., Jacobi, F., Rehm, J., Gustavsson, A., Svensson, M., Jonsson, B., Olesen, J., Allgulander, C., Alonso, J., Faravelli, C., Fratiglioni, L., Jennum, P., Lieb, R., Maercker, A., van Os, J., Preisig, M., Salvador-Carulla, L., Simon, R., Steinhausen, H.C., 2011. The size and burden of mental disorders and other disorders of the brain in Europe 2010. Eur Neuropsychopharmacol. 21 (9), 655-679.

Yildiz-Yesiloglu, A., Ankerst, D.P., 2006. Neurochemical alterations of the brain in bipolar disorder and their implications for pathophysiology: a systematic review of the 
in vivo proton magnetic resonance

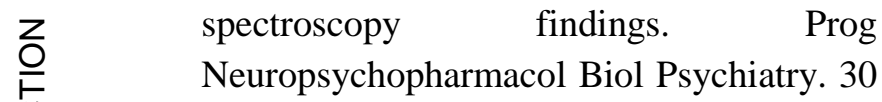
(6), 969-995.

Yoshimura, M., Koenig, T., Irisawa, S., Isotani, T., Yamada, K., Kikuchi, M., Okugawa, G., Yagyu, T., Kinoshita, T., Strik, W., Dierks, T., 2007. A pharmaco-EEG study on antipsychotic drugs in healthy volunteers. Psychopharmacology (Berl) 191 (4), 9951004.

Yuksel, C., Ongur, D., 2010. Magnetic resonance spectroscopy studies of glutamate-related abnormalities in mood disorders. Biol Psychiatry. 68 (9), 785-794. 


\title{
CHAPTER 2
}

\section{Decrease Of Theta Response In Euthymic Bipolar Patients During An Oddball Paradigm}

\author{
M.I. Atagün, B. Güntekin, A. Özerdem, E. Tülay, E. Başar
}

Cognitive Neurodynamics $2013 ; 7: 213-223$ 


\section{Abstract}

Background: Theta oscillations are related to cognitive functions and reflect functional integration of frontal and medial temporal structures into coherent neurocognitive networks. This study assessed event-related theta oscillations in medication-free, euthymic patients with bipolar disorder upon auditory oddball paradigm.

Method: Twenty-two DSM-IV euthymic bipolar I $(n=19)$ and II $(n=3)$ patients and twenty-two healthy subjects were included. Patients were euthymic for at least 6 months, and psychotropic-free for at least 2 weeks. EEG was recorded at 30 electrode sites. Auditory oddball paradigm and sensory stimuli were used. Event-related Oscillations were analyzed using adaptive filtering in two different theta frequency bands $(4-6 \mathrm{~Hz}, 6-8 \mathrm{~Hz})$.

Results: In healthy subjects, slow theta $(4-6 \mathrm{~Hz})$ responses were significantly higher than those of euthymic patients upon target, non-target and sensory stimuli $(p<0.05)$. Fast theta $(6-8 \mathrm{~Hz})$ responses of healthy subjects were significantly higher than those of euthymic patients upon target-only stimuli $(p<0.05)$.

Conclusion: Reduced theta oscillations during auditory processing provide strong quantitative evidence of activation deficits in related networks in bipolar disorder. Fast theta responses are related to cognitive functions, whereas slow theta responses are related to sensory processes more than cognitive processes.

Keywords: Bipolar disorder, theta oscillations, medication-free, euthymia, cognitive dysfunction 


\section{Introduction}

Cognitive deficits and emotional dysregulation in euthymia are indicators of enduring pathology in bipolar disorder (BD). Disruptions of the connections between frontal cortex, amygdala, basal ganglia, thalamus, entorhinal cortex and hippocampus are suggested to participate in the underlying pathology of bipolar disorder (Dupont et al. 1995; Caligiuri et al. 2004; Blumberg et al. 2002; Philips et al. 2003; Strakowski et al. 2005). These connections are also believed to serve in modulation of cognition and emotional consonance (Strakowski et al. 2005).

In contrast to the wealth of neuroimaging studies in $\mathrm{BD}$, little is known about electrophysiologic correlates. Cognitive deficits in BD may indicate that EEG recorded under cognitive task conditions would be better suited to identify electrophysiological correlates of cognitive dysfunctions. Event-related potential studies of P300 peak amplitudes produced inconsistent results in BD; Some studies reported reduced P300 amplitudes (Muir et al. 1991; El-Badri et al. 2001; Salisbury et al. 1998; O’Donnell et al. 2003; O’Donnell et al. 2004b; Fridberg et al. 2009), while some others reported no difference between healthy controls and patients with BD (Souza et al. 1995; Strik et al. 1998; Hall et al. 2007; Kaya et al. 2007; Schulze et al. 2007, 2008).

Dysfunction in sensory or cognitive processes cannot be explained only by a frequency response; however, connectivity deficits between involved brain sites may be reflected in a frequency response (Başar 2006). Over the last decade, oscillatory activity has increasingly been applied in various clinical pathologies, including bipolar disorder (see reviews Başar and Güntekin 2008; Başar 2010). The degree of resting state long-range synchrony was found to be significantly reduced in manic patients in comparison to healthy controls at all frequencies (Bhattacharya 2001). Medicated euthymic patients had increased delta and decreased beta synchronization in the frontal sites (Chen et al. 2009). Patients in manic or mixed state were found to have deficits in auditory EEG synchronization in beta and gamma range activity during click entrainment paradigm (O’Donnell et al. 2004a). Gamma band power reduction has also been found in euthymia (Lee et al. 2010). Auditory steady state response (ASSR) is thought to be generated by neural networks, including auditory cortices and thalamo-cortical circuits (Pantev et al. 1996; Rass et al. 2010). Deficits in generation and maintenance of ASSR in bipolar disorder may indicate disturbances in neural networks involved in auditory cortices (O'Donnell et al. 2004a, Rass et al. 2010). When comparing evoked neural oscillations in the 
left hemisphere in response to speech sounds, patients with BD displayed larger evoked oscillations than both schizophrenics and healthy controls in an MEG study (Oribe et al. 2010). In almost all of these studies, patients were medicated. Yener et al. (2007) showed that theta oscillations were significantly greater in patients with mild AD on cholinomimetic medication compared with those of medication-free patients with AD.

Previous studies by our group investigating oscillatory responses to visual oddball stimuli in medication-free bipolar patients found increased occipital beta activity in manic (Özerdem et al. 2008) and reduced long distance gamma coherence in manic (Özerdem et al. 2010) and euthymic states (Özerdem et al. 2011). Başar et al. (2012) recently showed that, in spontaneous EEG, bipolar patients had significantly reduced alpha activity in comparison to healthy controls.

Theta oscillations have been proposed to provide integration and communication between different brain areas (Başar 2010; Başar-Eroğlu and Demiralp 2001; Başar et al. 2001; Kirk and Mackay 2003; Sarnthein et al. 1998). Theta rhythm has been considered to be the fingerprint of all limbic structures; it is most prominent in the hippocampal formation (Lopes da Silva 1990). Theta oscillations are related to memory, attention and cognitive control processes (e.g., see Başar et al. 2001, Başar 1998, 1999; Klimesch 1999; Kahana et al. 1999), thus they are of particular interest in cognitive paradigms. Numerous structures in frontal (e.g., Gevins et al. 1997; Onton et al. 2005; Mitchell et al. 2008) and medial temporal regions (e.g., Başar-Eroglu et al. 1992; Kahana et al. 1999; Raghavachari et al. 2001; von Stein and Sarnthein 2000) generate cognition-related theta oscillations. Theta activity reflects functional integration of the abovementioned structures into coherent neurocognitive networks (see e.g. Başar et al. 2001; Başar 1998; Klimesch 1999; von Stein and Sarnthein 2000 for reviews). Thus, altered theta responses are likely to represent neurophysiologic correlates of cognitive deficits in BD (Sakowitz et al. 2000).

To our knowledge, no studies to date have compared the theta band power of control and BD samples. Electrophysiological assessments of oscillations provide high temporal resolution and therefore assessments of oscillatory responses to sensory or cognitive events constitute a useful imaging modality. Assessment of brain responses in the absence of any potential symptom or medication-related confounding effects may provide a major advantage to understand the underlying pathophysiology of bipolar disorder. The aim of this study was to assess evoked and event-related oscillatory responses to auditory stimuli in medication-free euthymic bipolar 
patients in comparison to healthy controls. Since verbal learning and verbal memory deficits are the most consistent cognitive dysfunctions in BD (Robinson et al. 2006; Bora et al. 2009), it can be hypothesized that theta responses to auditory oddball paradigm within the patient group may differ from healthy controls.

\section{Method}

\subsection{Subjects}

Twenty-two euthymic, drug-free patients with euthymic bipolar I $(n=19)$ or bipolar II $(n=3)$ diagnoses (female/male $=16 / 6$; mean age \pm SD: $31.18 \pm 6.34$, range: $23-44$ years), and sex (female/male: 16/6), age (mean age of healthy controls: $29.41 \pm 7.77$, range $=20-45$ ) and education (mean years of education for bipolar patients $=12.7 \pm 3.9 \mathrm{vs}$. healthy controls $=14.1 \pm 1.7$ years) -matched healthy controls were enrolled into the study (Table 1). All subjects were interviewed with the Turkish version of the SCID-I (Structured Interview according to DSMIV) (First et al. 1996). The local Ethical Committee of Bakırkoy Research and Training Hospital approved the study. Each participant provided written informed consent. Patients needed to be euthymic at least for 6 months, psychotropic-free for at least two weeks prior to study enrollment; to score 7 or less on the reliable and validated Turkish versions of the Young Mania Rating Scale (YMRS) (Young et al. 1978; Karadağ et al. 2002), Hamilton Depression Rating Scale (HAM-D 21) (Hamilton 1960; Aydemir and Deveci 2003); to have no co-morbid axis I diagnosis, and to be medically healthy, as confirmed by physical examination and routine laboratory tests. Exclusion criteria were: Pregnancy, lactation, consumption of alcohol or illicit substances within the previous 2 weeks, history of alcohol- or substance misuse, axis 1 psychiatric comorbidity and neurological conditions such as neurodegenerative diseases, epilepsy, and brain surgery. Volunteers who proved to have no present or past psychiatric condition on SCID-I interview and to be medically healthy on physical examination were enrolled as the control group.

\subsection{Stimuli and Paradigms}

Participants were seated in a dimly-lit isolated room with eyes open. Two types of stimuli were presented: simple auditory stimuli for analyzing auditory evoked potentials (AEP), and auditory oddball paradigm for analyzing auditory event-related potentials (AERP). The auditory stimuli had $16 \mathrm{~ms}$ rising time, $50 \mathrm{~ms}$ falling time and $1000 \mathrm{~ms}$ duration, and were presented by two loudspeakers. 
- The auditory simple stimuli were tones of $80 \mathrm{~dB}$ and $1500 \mathrm{~Hz}$. The inter-stimulus intervals varied randomly between 3 and 7 seconds. The total number of stimuli was 60 .

- The classical auditory oddball paradigm that was used in the experiments consisted of two types of stimuli: task-relevant target and task- irrelevant non-target (standard). The total number of stimuli was 120 (40 target, 80 non-target). Target ( $80 \mathrm{~dB}, 1600-\mathrm{Hz}$ tones) and non-target (1500-Hz tones) were presented in a random sequence. The interval between tones varied randomly between 3 and 7 seconds. The subjects were instructed to keep a mental count of the number of target $(1600 \mathrm{~Hz})$ tones.

The evoked and event-related theta responses to the target, non-target and simple auditory stimulation stimuli were analyzed and compared.

\subsection{Electrophysiological Recording}

EEG was recorded with $30 \mathrm{Ag}-\mathrm{AgCl}$ electrodes mounted in an elastic cap (Easy-cap) according to the international 10-20 system. Additionally, two linked earlobe electrodes $(\mathrm{A} 1+\mathrm{A} 2)$ served as references. The EOG from the medial upper and lateral orbital rim of the right eye was also registered. For the reference electrodes and EOG recordings, $\mathrm{Ag}-\mathrm{AgCl}$ electrodes were used. All electrode impedances were less than $10 \mathrm{k} \Omega$. The EEG was amplified by means of a BrainAmp 32-channel DC device with band limits of 0.01-250 Hz. The EEG was digitized online at a sampling rate of $500 \mathrm{~Hz}$.

\subsection{Evoked and Event-related Oscillatory Analysis by Means of Adaptive Filtering.}

Artifacts were eliminated by manual off-line selective averaging, taking into consideration the EOG recorded from the right eye. The sweep numbers were equalized randomly between the target, non-target and simple auditory stimulation conditions. The epochs (between 0 and 800 $\mathrm{ms}$ ) of each subject were averaged and then the digital FFT-based power spectrum analysis was performed. (10\% Hanning windowing function was evaluated in order to calculate the theta frequency peak). Subject averages and grand averages were calculated for each electrode site and experimental condition. As seen in Figure 1, in the grand average of response power spectrum upon stimulation of target stimuli, two different peaks were detected in theta frequency in the healthy control group, both for slow theta $(4-6 \mathrm{~Hz})$ and fast theta $(6-8 \mathrm{~Hz})$. Adaptive filtering was applied in analyzing the data in both healthy and patient groups, due to the two different peaks observed in theta frequency range only in healthy controls. Adaptive 
filtering of the response provides a major advantage that subsystems of the system might be selectively removed to obtain isolation. Separate isolation of the filters may allow the choice of amplitude and frequency characteristics of the filters. Ideal filters may be applied without phase shifts. Furthermore, this method also permits the definition of filters with exact characteristics their adequate regulation according to the amplitude characteristics of the system (for further information see Başar 2004). Accordingly, each subject's averaged evoked and event-related potentials were digitally filtered in slow theta $(4-6 \mathrm{~Hz})$ and fast theta $(6-8 \mathrm{~Hz})$ frequency ranges. The maximum peak-to-peak amplitudes for each subject's averaged slow theta (4-6 $\mathrm{Hz})$ and fast theta $(6-8 \mathrm{~Hz})$ responses were analyzed; that is, the largest peak-to-peak value in these frequency ranges in terms of $\mu \mathrm{Vs}$ found in the time window between 0 and $500 \mathrm{~ms}$.

Figure 1. Power spectrum of auditory evoked and event related responses over left frontal (F3) location
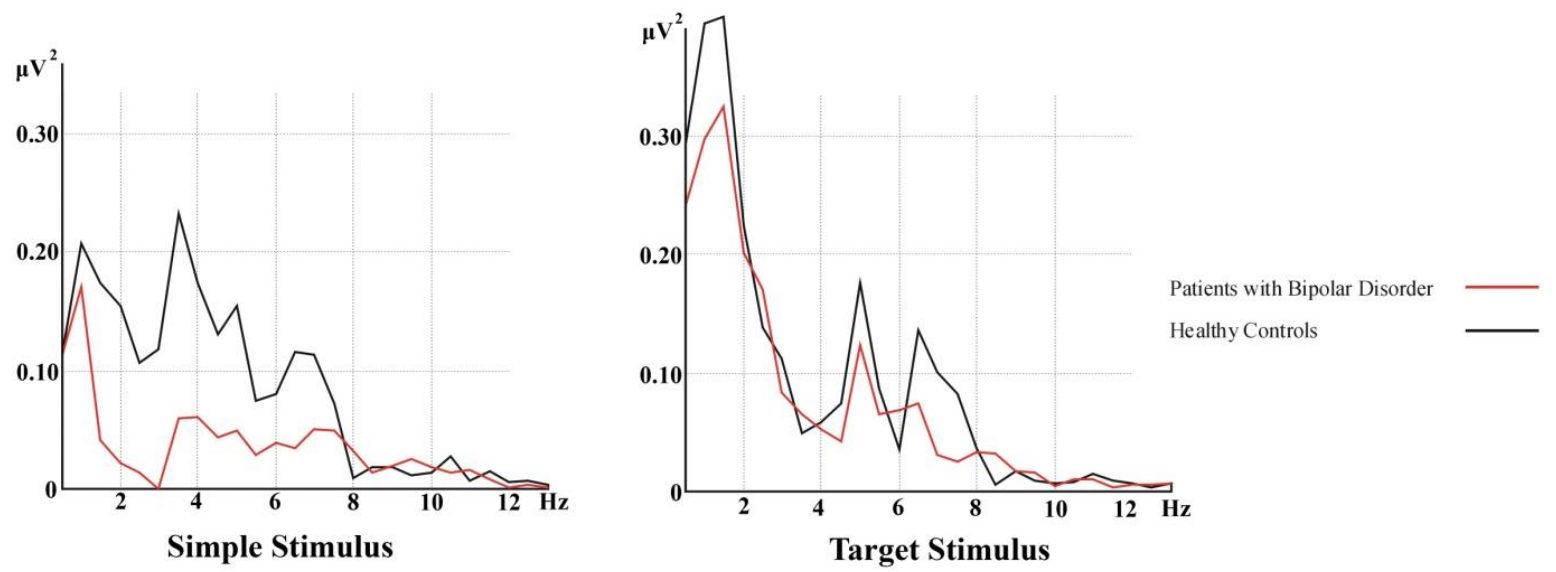

\subsection{Statistical Analysis}

SPSS was used for statistical analysis. A repeated measure ANOVA was used to determine the statistical significance of differential theta responses over different conditions, locations, and between patients and controls. Two separate ANOVAs were used for the two different frequency theta ranges $(4-6 \mathrm{~Hz}$ and $6-8 \mathrm{~Hz}$ ). In the analysis of theta responses, repeated measures of ANOVA included the healthy controls and euthymic patients as the betweensubjects factor; stimulus types (target, non-target, simple auditory stimulation) at three levels, locations [frontal $\left(\mathrm{F}_{3}-\mathrm{F}_{4}\right)$, central $\left(\mathrm{C}_{3}-\mathrm{C}_{4}\right)$, temporal $\left(\mathrm{T}_{7}-\mathrm{T}_{8}\right)$, temporo-parietal $\left(\mathrm{TP}_{7}-\mathrm{TP}_{8}\right)$, Parietal $\left(\mathrm{P}_{3}-\mathrm{P}_{4}\right)$, Occipital $\left.\left(\mathrm{O}_{1}-\mathrm{O}_{2}\right)\right]$ signals at six levels and hemispheres (right, left) at two levels were included as within-subject factors. Greenhouse-Geisser corrected p-values are reported. The t-test was used for post-hoc comparisons. In all analyses, the level of significance 
was $p<0.05$ with $95 \%$ confidence interval. In the analysis of behavioral data, due to extreme values, logarithmic transformation was applied to numbers of errors and Spearman's correlation analysis was used for correlations. Spearman's correlation test was used to obtain correlations between the clinical data and evoked and event-related theta oscillatory responses. Each subjects' frontal, central, temporal, temporo-parietal, parietal and occipital values were obtained by calculating average values of left and right electrode values and these averages were used to obtain correlations between clinical variables.

\section{Results}

Clinical characteristics of the patients are given in Table 1. All patients were drug-free for at least two weeks and euthymic for at least 6 months; mean score for the 21-item HAM-D was $2.55( \pm 2.3)$ and mean score for YMRS was $0.55( \pm 1.19)$.

Table 1. Subjects' characteristics

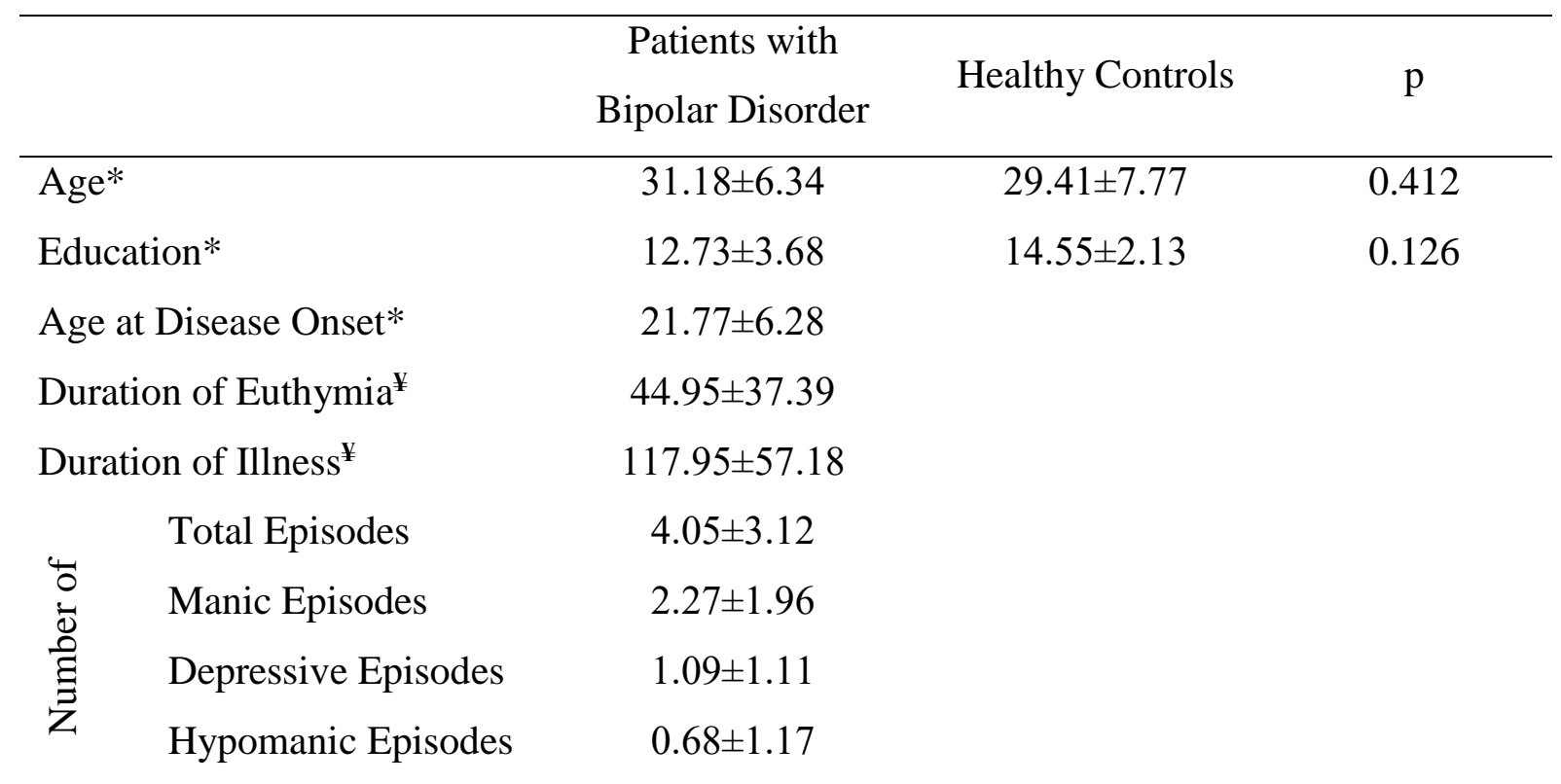

Mean $\pm S D$, *years, ¥months

Figure 2 shows a sample of filtered and averaged theta response at the left-frontal location. There was a $20 \%$ decrease in evoked oscillations and a $30 \%$ decrease in event-related oscillations in patients with bipolar disorder in comparison to healthy controls. 
Figure 2. Filtered theta response in left-frontal $\left(\mathrm{F}_{3}\right)$ electrode site upon simple and target stimuli. Grand averages of theta responses of patients with bipolar disorder are represented by red lines and those of healthy controls are represented by black lines. There is a $20 \%$ decrease in evoked oscillations and 30\% decreases in event-related oscillations in patients with bipolar disorder compared to healthy controls.

\section{4-6 Hz}
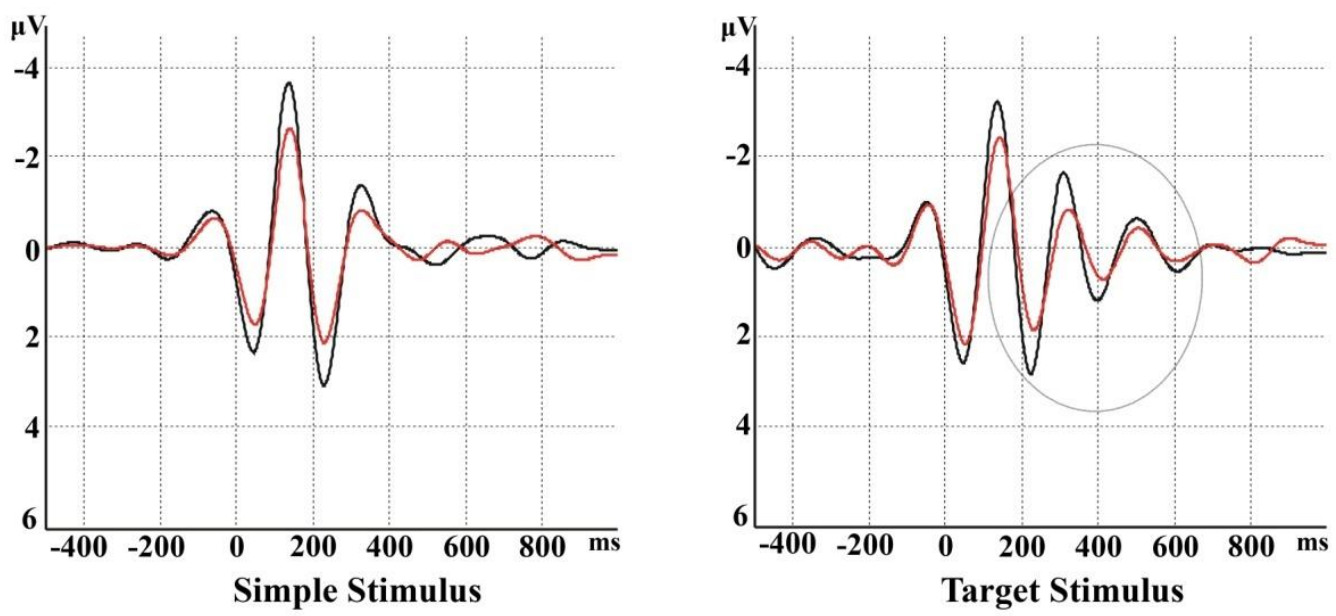

6-8 Hz
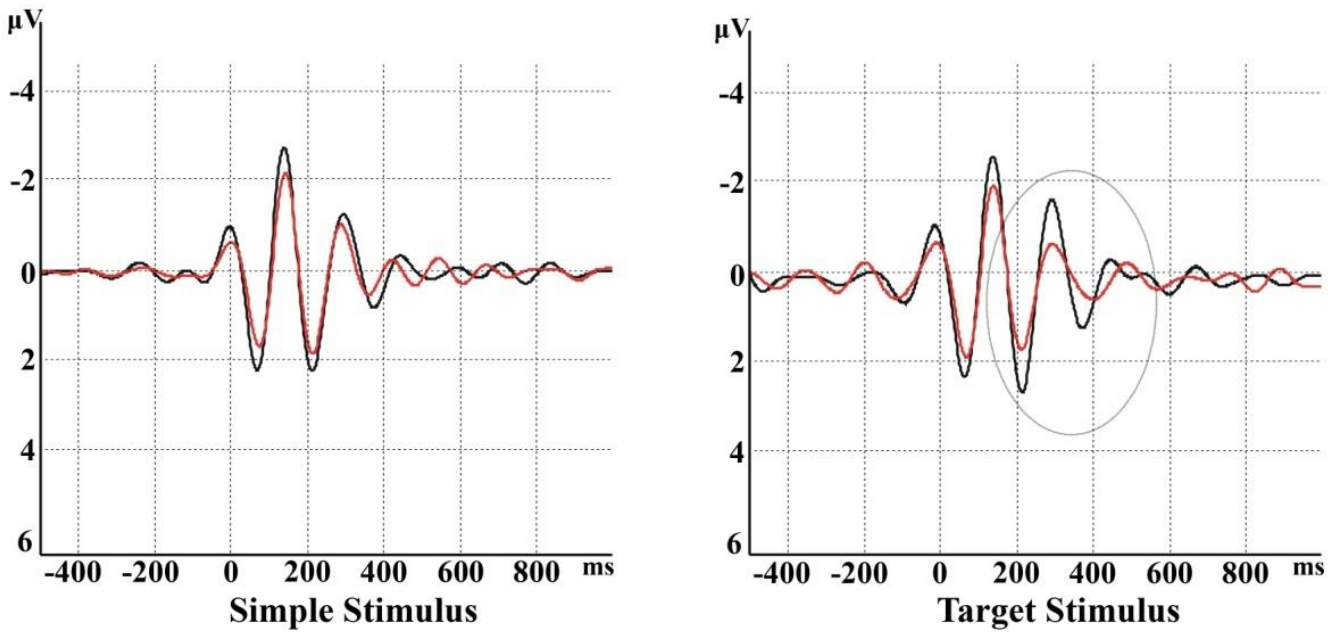

Patients with Bipolar Disorder

Healthy Controls

\subsection{Slow Theta Oscillations $(4-6 \mathrm{~Hz})$}

- General Features -

In the repeated measures of ANOVA, there was a significant stimulus-type effect $[F(2,84)$ : $17.672 ; \mathrm{p}<0.0001]$ in the whole group $(\mathrm{n}=44)$. Post-hoc comparisons showed that slow theta responses upon target stimuli were significantly higher than slow theta responses upon non- 
target stimuli in the whole group $(\mathrm{p}<0.0001)$. Furthermore, slow theta responses upon simple auditory stimuli were significantly higher than slow theta responses upon non-target stimuli $(\mathrm{p}<0.0001)$. No difference was detected between simple and target stimuli. The location effect was also significant [F(5,210): 126.738; $\mathrm{p}<0.0001]$ in the whole group $(n=44)$. Post-hoc comparisons showed that, regardless of the stimulus type, slow theta responses at frontal and central electrodes were higher than temporal, temporoparietal, parietal and occipital electrodes ( $\mathrm{p}<0.0001$ for all comparisons). Furthermore, slow theta responses at temporal and temporoparietal electrodes were higher than those at parietal and occipital electrodes $(\mathrm{p}<0.0001$ for all comparisons). There was a significant [location $\times$ stimulus-type] effect $(F(10,420)$ : 7.352; $\mathrm{p}<0.0001)$ in the whole group $(\mathrm{n}=44)$. Post-hoc comparisons showed that fast theta responses to target stimuli were significantly higher than fast theta responses to auditory nontarget stimuli at frontal, central and occipital electrode sites $(\mathrm{p}<0.002 ; \mathrm{p}<0.002 ; \mathrm{p}<0.0001)$. It is also note that in all electrodes slow theta responses of non-target stimuli were lower than the slow theta responses of target and simple auditory stimuli.

\section{- Comparison of the patient and healthy control groups -}

Slow theta response differed significantly between the patient and control groups $(F(1,42)$ : 5.686; $\mathrm{p}<0.05)$. The t-test showed that patients had significantly lower slow theta activity in response to simple stimuli (EP) at $\mathrm{F}_{3}(\mathrm{p}<0.01), \mathrm{F}_{4}(\mathrm{p}<0.01), \mathrm{C}_{3}(\mathrm{p}<0.01), \mathrm{C}_{4}(\mathrm{p}<0.05), \mathrm{T}_{7}$ ( $\mathrm{p}<0.05), \mathrm{T}_{8}(\mathrm{p}<0.05)$ and $\mathrm{P}_{3}(\mathrm{p}<0.05)$ electrodes. Also for the target stimulus, the patients had significantly lower values at the same locations $\left[F_{3}(p<0.05), F_{4}(p<0.05), C_{3}(p<0.05), C_{4}\right.$ $(p<0.05)]$. For the non-target stimuli, the difference was significant at $T_{7}(p<0.05)$ and $T_{8}$ $(\mathrm{p}<0.05)$ locations. Differences between groups are represented in Figure 3. 
Figure 3. Mean amplitudes of patients with bipolar disorder and healthy controls in 4-6 Hz frequency range. Red bars represent patients with bipolar disorder and blue bars represent healthy controls. “*” sign represent $p$ values less than 0.05 ; “ $\times ”$ sign represent $p$ values less than 0.001 .

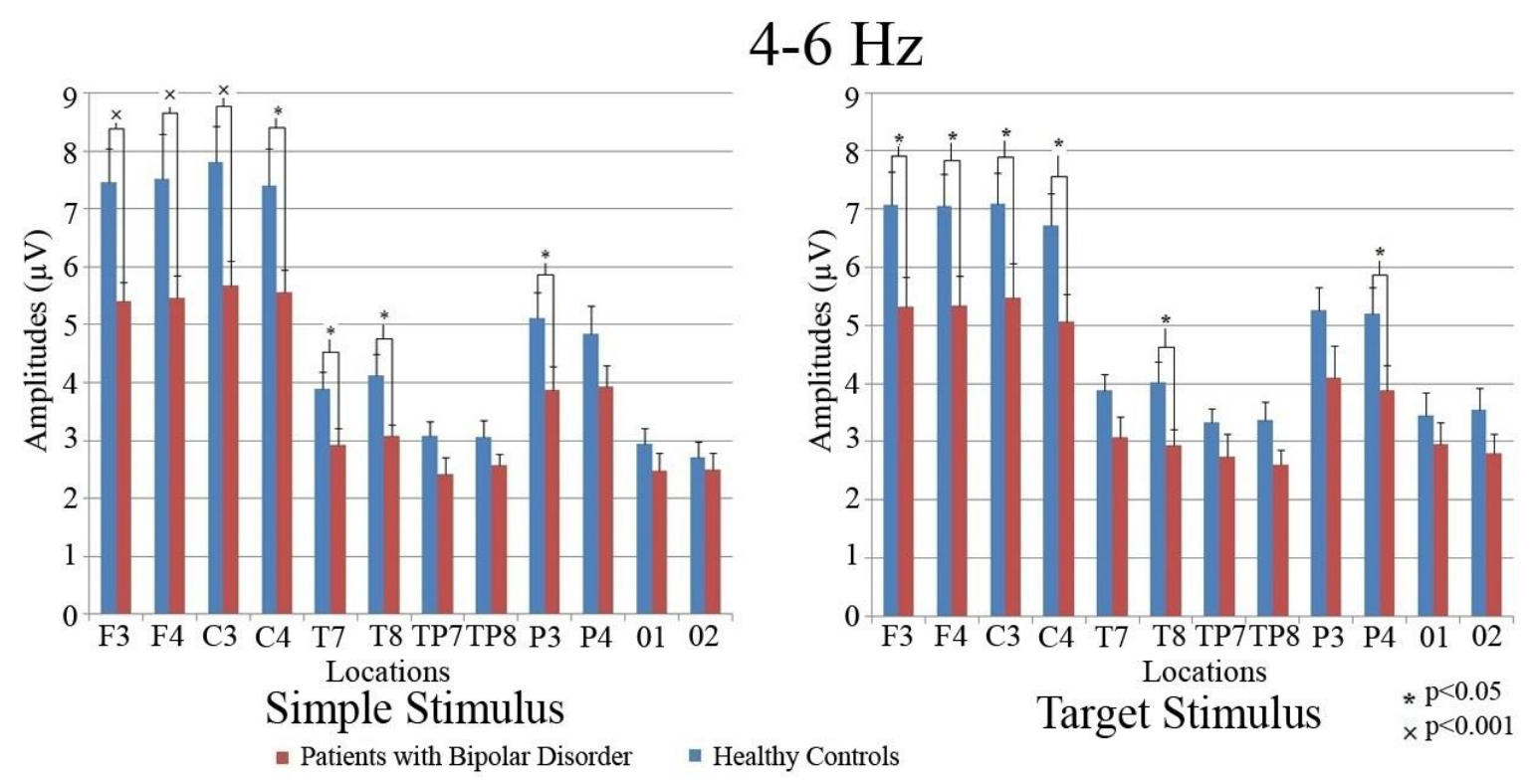

\subsection{Fast Theta Oscillations $(6-8 \mathrm{~Hz})$}

General Features -

ANOVA showed that fast theta responses differed significantly between stimulus-type effects $[F(2,84): 9.691 ; \mathrm{p}<0.0001]$ in the whole group $(\mathrm{n}=44)$. Post-hoc comparisons showed that fast theta responses upon target stimuli were significantly higher than fast theta responses upon nontarget stimuli $(\mathrm{p}<0.0001)$. Furthermore, fast theta responses upon simple auditory stimuli were significantly higher than fast theta responses upon non-target stimuli $(\mathrm{p}<0.0001)$. No significant difference was detected between simple and target stimuli. The location effect was also significant $[F(5,210): 93.298 ; \mathrm{p}<0.0001]$ in the whole group $(\mathrm{n}=44)$. Post-hoc comparisons showed that fast theta responses at frontal and central electrodes were higher than at temporal, temporoparietal, parietal and occipital electrodes ( $p<0.0001$ for all electrodes). Furthermore, fast theta responses at temporal and temporoparietal electrodes were higher than those at parietal and occipital electrodes ( $<<0.001$; for all comparisons). (Frontal=Central $>$ temporal $>$ temporoparietal $>$ Parietal $>$ Occipital $)$ There was a significant [location $\times$ stimulus-type] effect $[F(10,420): 2.882 ; \mathrm{p}<0.05]$ in the whole group $(\mathrm{n}=44)$. Posthoc comparisons showed that fast theta responses to target stimuli were significantly higher 
than fast theta responses to auditory non-target stimuli at frontal, central and occipital electrode sites ( $p<0.003 ; p<0.007 ; p<0.0001)$. It is also note that in all electrodes fast theta responses of non-target stimuli were lower than the fast theta responses of target and simple auditory stimuli.

\section{- Comparison of the patient and healthy control groups -}

The ANOVA of fast theta responses revealed significant [stimulus $\times$ location $\times$ group] effect [F(10.420): 2.867; $\mathrm{p}<0.05)$. Post-hoc comparisons showed that, upon simple stimuli, fast theta responses of healthy controls were greater than patients with BD only in right temporal region ( $<<0.01$ for all sites); upon target stimuli, fast theta responses of healthy controls were greater than patients with BD at frontal, central, right temporal and right parietal regions $(p<0.001$, $\mathrm{p}<0.001, \mathrm{p}<0.01$ and $\mathrm{p}<0.05$ respectively). Upon non-target stimuli, healthy controls showed greater fast theta responses than patients with $\mathrm{BD}$ only in the temporal region $(\mathrm{p}<0.05)$. Comparison of [stimuli $\times$ location $\times$ hemisphere] revealed that the responses of the healthy control group were significantly higher than the patient group [F(10.420): 2.093; $p<0.05]$. Ttest results showed that patients had significantly lower theta activity in response to simple stimuli (EP) only at site $\mathrm{T}_{8}(\mathrm{p}<0.01)$. In response to the target stimuli, the patients had significantly lower values at locations $F_{3}(p<0.05), F_{4}(p<0.01), C_{3}(p<0.05), C_{4}(p<0.01), T_{8}$ $(p<0.01)$ and $\mathrm{P}_{4}(\mathrm{p}<0.05)$. No significant difference was found between the groups in response to the non-target stimuli. Inter-group differences are represented in Figure 4.

Figure 4. Mean amplitudes of patients with bipolar disorder and healthy controls in $6-8 \mathrm{~Hz}$ frequency range. Red bars represent patients with bipolar disorder and blue bars represent healthy controls. "*” sign represent $p$ values less than 0.05 ; “ $\times$ ” sign represent $p$ values lower than 0.001 .

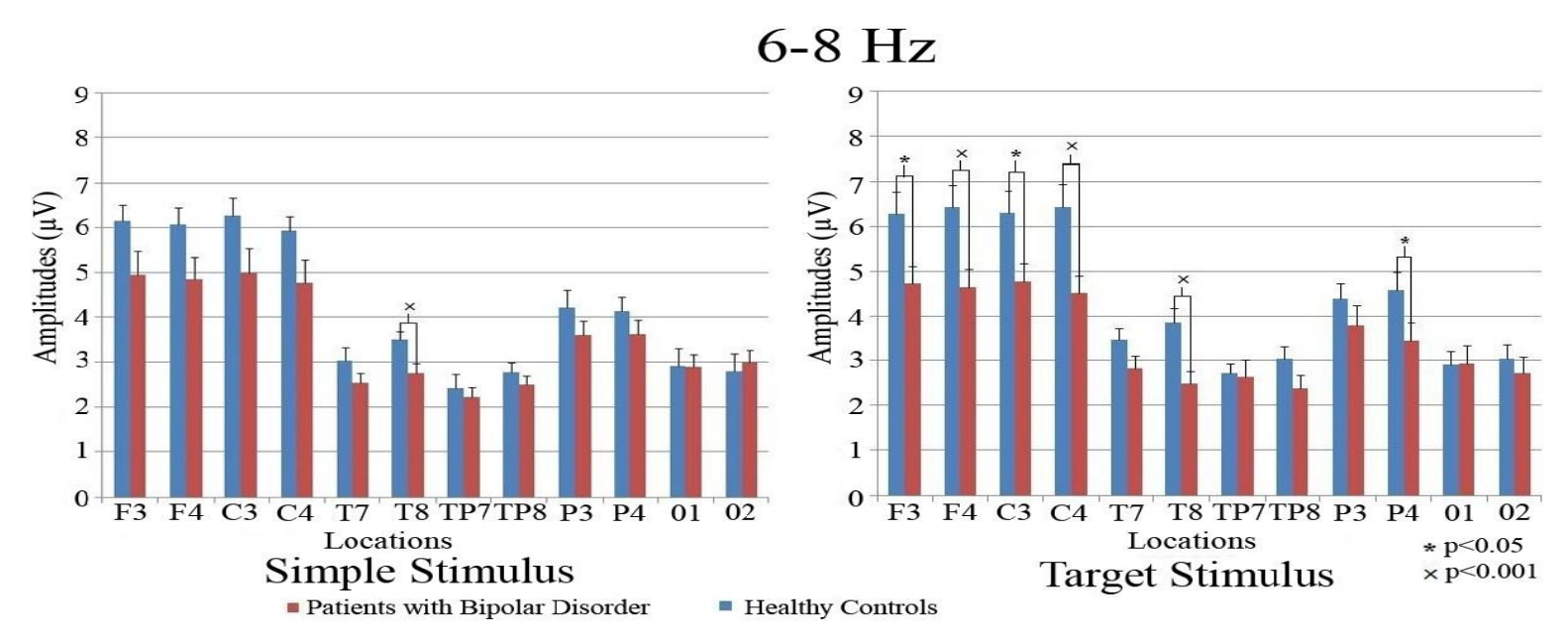




\subsection{Behavioral Data}

During the elicitation period of event-related oscillations, subjects were instructed to count the target stimuli as a cognitive task. It was found that 18 of 22 members of the patient group (mean=3.82 \pm 7.39 , range:1-26) made errors, compared with 11 of the 22 control subjects (mean=6.56 \pm 9.54 , range: $1-40$ ).

\subsection{Correlation Analyses}

Each subjects' frontal, central, temporal, temporo-parietal, parietal and occipital electrodes were used to obtain correlations between clinical variables such as age, education, age at disease onset, duration of euthymia, duration of the disease, total numbers of episodes, total numbers of manic and depressive episodes. Slow theta and fast theta responses to simple, target and nontarget stimuli correlated significantly with age and age at disease onset (Table 2).

Table 2. Correlations between mean amplitudes of averaged filtered theta response and clinical variables. Spearman's Correlation test; $r$ values. ${ }^{*} p<0.05$; ${ }^{\ddagger} p<0.001$. Education, age at disease onset, duration of euthymia, total numbers of episodes, total numbers of manic and depressive episodes did not show any correlation.

\begin{tabular}{|c|c|c|c|c|c|c|c|c|}
\hline & & & Frontal & Central & Temporal & $\begin{array}{c}\text { Temporo- } \\
\text { parietal }\end{array}$ & Parietal & Occipital \\
\hline \multirow{5}{*}{$\begin{array}{l}\text { Simple } \\
\text { Stimulus }\end{array}$} & \multirow{2}{*}{ 4-6 Hz } & Age & $-0.49^{*}$ & $-0.70^{F}$ & $-0.50^{*}$ & $-0.60^{F}$ & $-0.55^{Y}$ & $-0.43^{*}$ \\
\hline & & Age at Disease Onset & -0.40 & $-0.52^{*}$ & -0.13 & -0.36 & $-0.55^{\Psi}$ & $-0.49^{*}$ \\
\hline & \multirow{3}{*}{ 6-8 Hz } & Age & -0.33 & $-0.58^{\Psi}$ & -0.40 & -0.42 & -0.40 & -0.42 \\
\hline & & Age at Disease Onset & -0.28 & -0.38 & -0.05 & -0.17 & $-0.43^{*}$ & -0.33 \\
\hline & & Duration of Last Episode & 0.28 & 0.16 & $0.51^{*}$ & 0.34 & 0.06 & 0.14 \\
\hline \multirow{5}{*}{$\begin{array}{l}\text { Target } \\
\text { Stimulus }\end{array}$} & \multirow{2}{*}{ 4-6 Hz } & Age & $-0.53^{*}$ & $-0.53^{*}$ & $-0.51^{*}$ & -0.39 & $-0.53^{*}$ & -0.35 \\
\hline & & Age at Disease Onset & -0.26 & -0.30 & -0.21 & -0.22 & -0.40 & -0.13 \\
\hline & \multirow{3}{*}{ 6-8 Hz } & Age & -0.23 & $-0.50^{*}$ & $-0.42^{*}$ & $-0.50^{*}$ & $-0.43^{*}$ & -0.38 \\
\hline & & Age at Disease Onset & -0.17 & $-0.47^{*}$ & -0.23 & -0.30 & $-0.47^{*}$ & -0.25 \\
\hline & & Duration of Last Episode & 0.32 & 0.17 & 0.41 & 0.27 & 0.27 & 0.15 \\
\hline \multirow{5}{*}{$\begin{array}{l}\text { Non-target } \\
\text { Stimulus }\end{array}$} & \multirow{2}{*}{ 4-6 Hz } & Age & -0.36 & $-0.48^{*}$ & $-0.48^{*}$ & $-0.57^{\not}$ & $-0.54^{Y}$ & $-0.50^{*}$ \\
\hline & & Age at Disease Onset & $-0.43^{*}$ & $-0.52^{*}$ & -0.18 & $-0.44^{*}$ & $-0.61^{F}$ & -0.29 \\
\hline & \multirow{3}{*}{ 6-8 Hz } & Age & -0.16 & -0.28 & -0.35 & $-0.48^{*}$ & $-0.43^{*}$ & -0.41 \\
\hline & & Age at Disease Onset & -0.16 & -0.29 & -0.17 & -0.25 & -0.38 & -0.22 \\
\hline & & Duration of Last Episode & 0.32 & 0.30 & $0.44^{*}$ & 0.35 & 0.34 & 0.01 \\
\hline
\end{tabular}

Spearman's Correlation test. $r$ values. L: left, R: right, $n s$ : non-significant $* p<0.05$; ${ }^{¥} p<0.001$. Education, age at disease onset, duration of euthymia, total numbers of episodes, total numbers of manic and depressive episodes did not show any correlation. 


\section{Discussion}

The major finding of the present study is that patients with bipolar disorder showed significantly lower theta oscillatory responses upon auditory stimulation and in response to target and simple stimuli during an oddball paradigm in comparison to healthy controls. Patients presented different slow $(4-6 \mathrm{~Hz})$ and fast $(6-8 \mathrm{~Hz})$ theta response patterns. As the slow theta $(4-6 \mathrm{~Hz})$ responses of the patients were significantly lower upon simple auditory stimulation and target stimuli during the oddball paradigm at bilateral frontal, central, right temporal and right parietal regions, the $6-8 \mathrm{~Hz}$ activity of the patients showed significant reductions in the same locations compared to healthy controls only upon target stimulus.

A comparison of Figures 3 and 4 clearly imposes the following reasoning: in the slow theta frequency window (4-6 Hz), all cortical areas react with significant increase, which is independent of the modality of stimulation. Simple stimulation and target stimulation both show significant increases of as much as $50 \%$. In contrast, within the fast theta frequency band (6-8 Hz), all locations showed significant responses upon target stimulus; however, for except $\mathrm{T} 8$, none of the cortical areas are increased upon simple stimulation. These results clearly indicate that both theta responses are involved with different functional processing.

Theta frequency bands have been extensively studied and are believed to be involved in cognitive functions such as working memory (Başar-Eroğlu and Demiralp 2001; Başar et al. 2001; Başar 1998, 1999; Klimesch 1999; Kahana et al. 1999; Klimesch et al. 1996, 1997, $2001 \mathrm{a}$, 2001b). According to the model of Klimesch et al. (1996), short-term memory demands lead to synchronization in the theta band, manifested as an increase in band power, and occurs at the anterior limbic system. Oscillations in the theta and alpha band may provide the basis for encoding, accessing and retrieving cortical codes that are stored in the form of widely distributed but intensively interconnected cell assemblies (Başar 1999). In a recent study, Caravaglios and colleagues (2010) compared theta frequency responses (to oddball paradigm) of patients with Alzheimer disease and healthy controls by means of oddball paradigm. They found that although responses of healthy controls were responsive to target stimuli, theta frequency activity of the patients was not responsive to target or non-target stimuli. Patients showed increased pre-stimulus theta frequency activity, and no enhancement was detected in the early (0-250 ms) or late (250-500 ms) post-stimulus interval. The authors commented that patients had insufficient resources for adequate attention. It was also indicated that, unlike the healthy controls, patients did not have prominent frontal lobe activity during stimulus 
processing. Theta and delta are the most affected frequencies upon oddball paradigm in Alzheimer disease and mild cognitive impairment as instances of cognitive dysfunctions (Başar et al. 2010). The authors suggested that cognitive impairment in Alzheimer disease was particularly manifested by reduced coherences in delta and theta frequency ranges.

Event-related oscillations in the theta band are prolonged and/or have a second time-window approximately $300 \mathrm{~ms}$ after the presentation of the target stimulus in oddball experiments (Başar-Eroğlu and Demiralp 2001; Başar et al. 2001). Prolongation of theta response is interpreted as the reflection of the correlation with selective attention. Başar-Eroğlu and Demiralp (2001) further showed that the second theta window is more associated with target stimuli. In addition, mental count of the target stimuli is associated with sustained attention and working memory. Patients with bipolar disorder are known to suffer from cognitive dysfunctions, particularly in sustained attention, executive functions, working memory, verbal learning and verbal memory sub-domains of cognitive functions (see meta-analyses, Robinson et al. 2006; Bora et al. 2009).

In general, theta frequency responses upon visual stimuli increase diffusely, including frontal, parietal, occipital and vertex sites; in contrast, auditory stimulus increases theta responses at frontal and parietal sites (Başar 1999; Demiralp and Başar 1992). The CA3 layers of the hippocampus, frontal- and parietal lobes are interconnected. The CA3 layer of hippocampus is shown to produce theta oscillations upon cognitive functions such as attention, perception, learning and memory in intracranial recordings from the cat brain (Başar-Eroğlu and Başar 1991). Distribution of theta responses at frontal and parietal lobes upon auditory stimulus may also be related to the hippocampus (Başar 1999). Therefore, these findings are strong quantitative indicators of dysfunctional cognitive processes occurring in cortico-subcortical loops in bipolar disorder. Başar-Eroğlu and colleagues (2008) showed that distribution of theta and alpha responses upon a visual paradigm had a different pattern from healthy controls in schizophrenia. Healthy controls' responses upon simple and non-target stimuli were distributed over the occipital lobe, while patients' responses were distributed over fronto-central sites. The authors' comment this on altered topography of the late theta response was that, even with simple task, patients required high cognitive effort to process stimuli.

Functional neuroimaging studies showed association between discrimination of auditory stimulation and frontoparietal activation, particularly over the right hemisphere (Paquette et al. 1996; Boucher and Bryden 1997; Zatorre 2001). In 6-8 Hz responses, the difference between 
groups became more prominent in response to target stimuli on the right hemisphere, which is a marker of activation deficit among patients with bipolar disorder when perceiving and discriminating pitch. Hence, the decreased theta responses of the patients indicate disruptions in the frontoparietal networks. In addition, these results suggest that, rather than 4-6 Hz, the 6$8 \mathrm{~Hz}$ band is more specific to the cognitive components of the oddball paradigm.

According to Yener and Başar (2010), sensory event may evoke brain areas reacting sensory inputs, whereas cognitive processes may evoke areas to respond to both sensory and cognitive inputs. According to this assumption, 4-6 Hz may include both sensory and cognitive components, whereas $6-8 \mathrm{~Hz}$ may include only cognitive components. In our study, the $4-6 \mathrm{~Hz}$ responses of the patient group were significantly decreased upon both simple and target stimuli; however, 6-8 Hz responses differed only upon target stimulus. Therefore, 4-6 Hz may be related to sensory events (Aftanas et al 2001a, 2001b, 2003a, 2003b), while 6-8 Hz may be related to cognitive processing (Aftanas et al. 2003b). Previous studies found that $4-6 \mathrm{~Hz}$ is more prominent in posterior sites, while $6-8 \mathrm{~Hz}$ is greater in frontal sites (Kamarajan et al. 2008; Krause et al. 2000). Loops integrating hippocampus and prefrontal cortices may serve cognitive functions via $6-8 \mathrm{~Hz}$ activity.

Several previous studies by our group reported that patients with bipolar disorder showed activation and synchronization deficits in different frequency ranges including delta, alpha, beta and gamma bands (Özerdem et al. 2008, 2010, 2011; Başar et al. 2012). This is the first study in the theta frequency range. A major strength of this study is the inclusion of medication-free patients, whereas the relatively small sample size is a limitation.

\section{Concluding Remarks}

The results of this study represent a specific feature for BD: Auditory processing deficiency may indicate disruption of synchronization during auditory cognitive activity. These findings provide neurophysiological evidence of auditory processing dysfunction in BD. On the other hand, the oscillatory brain dynamics of patients with BD differ from healthy controls in both auditory and visual paradigms.

The results suggest that fast theta $(6-8 \mathrm{~Hz})$ frequency responses are associated with cognitive functions, and that slow theta $(4-6 \mathrm{~Hz})$ frequency responses are more closely associated with sensory functions than with cognitive functions. Theta frequency should be analyzed in two different bands, including 4-6 and 6-8 Hz bands. 


\section{References}

Aftanas, L., Varlamov, A., Pavlov, S., Makhnev, V., Reva, N., 2001a. Eventrelated synchronization and desynchronization during affective processing: emergence of valence-related time-dependent hemispheric asymmetries in theta and upper alpha band. Int J Neurosci. 110:197-219.

Aftanas, L.I., Golocheikine, S.A., 2001b. Human anterior and frontal midline theta and lower alpha reflect emotionally positive state and internalized attention: highresolution EEG investigation of meditation. Neurosci Lett. 310:57-60.

Aftanas, L.I., Pavlov, S.V., Reva, N.V., Varlamov, A.A., 2003a. Trait anxiety impact on the EEG theta band power changes during appraisal of threatening and pleasant visual stimuli. Int J Psychophysiol. 50:205-212.

Aftanas, L.I., Varlamov, A.A., Reva, N.V., Pavlov, S.V., 2003b. Disruption of early event-related theta synchronization of human EEG in alexithymics viewing affective pictures. Neurosci Lett. 340:57-60.

Aydemir, Ö., Deveci, A., 2003. Validity and Reliability of Structured Interview for Hamilton Depression Rating Scale Seasonal Affective Disorders Version. VIIth Annual Spring Symposium of Psychiatric Association of Türkiye Abstract book p187.

Başar, E., 1998. Brain oscillations I: principles and approaches. Springer, Heidelberg.

Başar, E., 1999. Brain function and oscillations: II. Integrative brain function. Neurophysiology and cognitive processes. Springer, Heidelberg.

Başar, E., 2004. Memory and brain dynamics: oscillations integrating attention, perception, learning and memory. CRC Press, Florida.
Başar, E., 2006. The theory of whole-brainwork. Int J Psychophysiol. 60:133-138.

Başar, E., 2010. Why the Concept of "Quantum Brain" was not Discovered in 1940s. NeuroQuantology. 8:322-336.

Başar, E., Güntekin, B., 2008. A review of brain oscillations in cognitive disorders and the role of neurotransmitters. Brain Res. 1235:172-93.

Başar, E., Schürmann, M., Sakowitz, O., 2001. The selectively distributed theta system: functions. Int J Psychophysiol. 39:197-212.

Başar, E., Güntekin, B., Tülay, E., Yener, G.G., 2010. Evoked and Event-related Coherence of Alzheimer Patients Manifest Differentiation of Sensory-Cognitive Networks. Brain Res. 1357:79-90.

Başar. E., Güntekin, B., Atagün, İ., Turp Gölbaşı, B., Tülay, E., Özerdem, A., 2012. Brain's alpha activity is highly reduced in euthymic bipolar disorder patients. Cogn Neurodyn. 6:11-20.

Başar-Eroğlu, C., Başar, E., 1991. A compound $\mathrm{P} 300-40 \mathrm{~Hz}$ response of the cat hippocampus. Int J Neurosci. 60:227-237.

Başar-Eroğlu, C., Demiralp, T., 2001. Eventrelated theta oscillations: an integrative and comparative approach in the human and animal Brain. Int J Psychophysiol. 39:167195.

Başar-Eroğlu, C., Başar, E., Demiralp, T., Schürmann, M., 1992. P300-response: possible psychophysiological correlates in delta and theta frequency channels. A review. Int J Psychophysiol. 13:161-179.

Başar-Eroğlu, C., Schmiedt-Fehr, C., Marbach, S., Brand, A., Mathes, B., 2008. Altered 
oscillatory alpha and theta networks in schizophrenia. Brain Res. 1235:143-152.

Bhattacharya, J., 2001. Reduced degree of longrange phase synchrony in pathological human brain. Acta Neurobiol Exp (Wars). 61:309-318.

Blumberg, H.P., Charney, D.S., Krystal, J.H., 2002. Frontotemporal neural systems in bipolar disorder. Semin Clin Neuropsychiatry. 7:243-254.

Bora, E., Yucel, M., Pantelis, C., 2009. Cognitive endophenotypes of bipolar disorder: A meta-analysis of neuropsychological deficits in euthymic patients and their first-degree relatives. J. Affect. Disord. 113:1-20.

Boucher, R., Bryden, M.P., 1997. Laterality effects in the processing of melody and timbre. Neuropsychologia. 35:1467-1473.

Caligiuri, M.P., Brown, G.G., Meloy, M.J., Eyler, L.T., Kindermann, S.S., Eberson, S., Frank, L.R., Lohr, J.B., 2004. A functional magnetic resonance imaging study of cortical asymmetry in bipolar disorder. Bipolar Disord. 6:183-196.

Caravaglios, G., Castro, G., Costanzo, E., Di Maria, G., Mancuso, D., Muscoso, E.G., 2010. $\theta$ power responses in mild Alzheimer's disease during an auditory oddball paradigm: lack of theta enhancement during stimulus processing. J Neural Transm. 117:1195-1208.

Chen, A.C., Tang, Y., Rangaswamy, M., Wang, J.C., Almasy, L., Foroud, T., Edenberg, H.J., Hesselbrock, V., Nurnberger, J. Jr, Kuperman, S., O'Connor, S.J., Schuckit, M.A., Bauer, L.O., Tischfield, J., Rice, J.P., Bierut, L., Goate, A., Porjesz, B., 2009. Association of single nucleotide polymorphisms in a glutamate receptor gene (GRM8) with theta power of event-related oscillations and alcohol dependence. Am J
Med Genet B Neuropsychiatr Genet. 150:359-368.

Demiralp, T., Başar, E., 1992. Theta rhythmicities following expected visual and auditory targets. Int J Psychophysiol. 13:147-160.

Dupont, R.M., Jernigan, T.L., Heindel, W., Butters, N., Shafer, K., Wilson, T., Hesselink, J., Gillin, J.C., 1995. Magnetic resonance imaging and mood disorders. Localization of white matter and other subcortical abnormalities. Arch Gen Psychiatry. 52:747-755.

El-Badri, S.M., Ashton, C.H., Moore, P.B., Mursh, V.R., Ferrier, I.N., 2001. Electrophysiological and cognitive function in young euthymic patients with bipolar affective disorder. Bipolar Disord. 3:79-87.

First, M.B., Gibbon, M., Spitzer, R.L., Gibbon, M., Williams, J.B.W., 1996. User's guide for the structured interview for DSM-IV axis I disorders-research version (SCID-I, version 2.0, February 1996 final version). Biometrics Research, New York.

Fridberg, D.J., Hetrick, W.P., Brenner, C.A., Shekhar, A., Steffen, A.N., Malloy, F.W., O'Donnell, B.F., 2009. Relationships between auditory event-related potentials and mood state, medication, and comorbid psychiatric illness in patients with bipolar disorder. Bipolar Disord. 11:857-866.

Gevins, A., Smith, M.E., McEvoy, L., Yu, D., 1997. High-resolution EEG mapping of cortical activation related to working memory: effects of task difficulty, type of processing, and practice. Cereb Cortex. 7:374-385.

Hall, M.H., Rijsdijk, F., Picchioni, M., Schulze, K., Ettinger, U., Toulopoulou, T., Bramon, E., Murray, R.M., Sham, P., 2007. Substantial shared genetic influences on 
schizophrenia and event-related potentials. Am J Psychiatry. 164:804-812.

Hamilton, M., 1960. A rating scale for depression. J Neurol Neurosurg Psychiatry. 23:56-62.

Kahana, M.J., Sekuler, R., Caplan, J.B., Kirschen, M., Madsen, J.R., 1999. Human theta oscillations exhibit task dependence during virtual maze navigation. Nature. 399:781-784.

Kamarajan, C., Rangaswamy, M., Chorlian, D.B., Manz, N., Tang, Y., Pandey, A.K., Roopesh, B.N., Stimus, A.T., Porjesz, B., 2008. Theta oscillations during the processing of monetary loss and gain: a perspective on gender and impulsivity. Brain Res. 1235:45-62.

Karadă̆, F., Oral, E.T., Yalçın, F.A., Erten, E., 2002. Validity, Reliability of young mania rating scale in Turkey. Turk Psikiyatri Derg. 13:107-114.

Kaya, E., Aydemir, O., Selcuki, D., 2007. Residual symptoms in bipolar disorder: the effect of the last episode after remission. Biol Psychiatry. 31:1387-1392.

Kirk, I.J., Mackay, J.C., 2003. The role of thetarange oscillations in synchronising and integrating activity in distributed mnemonic networks. Cortex. 39:993-1008.

Klimesch, W., Doppelmayr, M., Pachinger, T., Ripper, B., 1997. Brain oscillations and human memory: EEG correlates in the upper alpha and theta band. Neurosci Lett. 238:912.

Klimesch, W., Doppelmayr, M., Russegger, H., Pachinger, T., 1996. Theta band power in the human scalp EEG and the encoding of new information. Neuroreport. 7:1235-1240.

Klimesch, W., 1999. EEG alpha and theta oscillations reflect cognitive and memory performance: a review and analysis. Brain Res Brain Res Rev. 29:169-195.

Klimesch, W., Doppelmayr, M., Stadler, W., Pöllhuber, D., Sauseng, P., Röhm, D., 2001a. Episodic retrieval is reflected by a process specific increase in human electroencephalographic theta activity. Neurosci Lett. 302:49-52.

Klimesch, W., Doppelmayr, M., Yonelinas, A., Kroll, N.E., Lazzara, M., Röhm, D., Gruber, W., 2001b. Theta synchronization during episodic retrieval: neural correlates of conscious awareness. Brain Res Cogn Brain Res. 12:33-38.

Krause, C.M., Sillanmäki, L., Koivisto, M., Saarela, C., Häggqvist, A., Laine, M., Hämäläinen, H., 2000. The effects of memory load on event-related EEG desynchronization and synchronization. Clin Neurophysiol. 111:2071-2078.

Lee, P.S., Chen, Y.S., Hsieh, J.C., Su, T.P., Chen, L.F., 2010. Distinct neuronal oscillatory responses between patients with bipolar and unipolar disorders: a magnetoencephalographic study. J Affect Disord. 123:270-275.

Lopes da Silva, F.H., 1990. A critical review of clinical applications of topographic mapping of brain potentials. J Clin Neurophysiol. 7:535-551.

Mitchell, D.J., McNaughton, N., Flanagan, D., Kirk, I.J., 2008. Frontal-midline theta from the perspective of hippocampal "theta". Prog Neurobiol. 86:156-185.

Muir, W.J., StClair, D.M., Blackwood, D.H., 1991. Long-latency auditory event-related potentials in schizophrenia and in bipolar and unipolar affective disorder. Psychol Med. 21:867-879.

O'Donnell, B.F., Hetrick, W.P., Vohs, J.L., Krishnan, G.P., Carroll, C.A., Shekhar, A., 
2004a. Neural synchronization deficits to auditory stimulation in bipolar disorder. Neuroreport. 15:1369-1372.

O’Donnell, B.F., Vohs, J.L., Hetrick, W.P., Carroll, C.A., Shekhar, A., 2004b. Auditory event-related potential abnormalities in bipolar disorder and schizophrenia. Int $\mathbf{J}$ Psychophysiology. 53:45-55.

O’Donnell, T.O., Rotzinger, S., Ulrich, M., Hanstock. C.C., Nakashima, T.T., Silverstone, P.H., 2003. Effects of chronic lithium and sodium valproate on concentrations of brain amino acids. Eur Neuropsychopharmacol. 13:220-227.

Onton, J., Delorme, A., Makeig, S., 2005. Frontal midline EEG dynamics during working memory. Neuroimage. 27:341-356.

Oribe, N., Onitsuka, T., Hirano, S., Hirano, Y., Maekawa, T., Obayashi, C., Ueno, T., Kasai, K., Kanba, S., 2010. Differentiation between bipolar disorder and schizophrenia revealed by neural oscillation to speech sounds: an MEG study. Bipolar Disord. 12:804-812.

Özerdem, A., Güntekin, B., Atagün, I., Turp, B., Başar, E., 2011. Reduced long distance gamma $(28-48 \mathrm{~Hz})$ coherence in euthymic patients with bipolar disorder. J Affect Disord. 132:325-332.

Özerdem, A., Güntekin, B., Saatçi, E., Tunca, Z., Başar, E., 2010. Disturbance in long distance gamma coherence in bipolar disorder. Prog Neuropsychopharmacol Biol Psychiatry. 34:861-865.

Özerdem, A., Güntekin, B., Tunca, Z., Başar, E., 2008. Brain oscillatory responses in patients with bipolar disorder manic episode before and after valproate treatment. Brain Res. 1235:98-108.

Pantev, C., Roberts, L.E., Elbert, T., Ross, B., Wienbruch, C., 1996. Tonotopic organization of the sources of human auditory steady-state responses. Hear Res. 101:62-74.

Paquette, C., Bourassa, M., Peretz, I., 1996. Left-ear advantage in pitch perception of complex tones without energy at the fundamental frequency. Neuropsychologia. 34:153-157.

Phillips, M.L., Drevets, W.C., Rauch, S.L., Lane, R.D., 2003. The neurobiology of emotion perception I: towards an understanding of the neural basis of normal emotion perception. Biol Psychiatry. 54:504-514.

Raghavachari, S., Kahana, M.J., Rizzuto, D.S., Caplan, J.B., Kirschen, M.P., Bourgeois, B., Madsen, J.R., Lisman, J.E., 2001. Gating of human theta oscillations by a working memory task. J Neurosci. 21:3175-3183.

Rass, O., Krishnan, G., Brenner, C.A., Hetrick, W.P., Merrill, C.C., Shekhar, A., O'Donnell, B.F., 2010. Auditory steady state response in bipolar disorder: relation to clinical state, cognitive performance, medication status, and substance disorders. Bipolar Disord. 12:793-803.

Robinson L.J., Thompson, J.M., Gallagher, P., Goswami, U., Young, A.H., Ferrier, I.N., Moore, P.B., 2006 A meta-analysis of cognitive deficits in euthymic patients with bipolar disorder. J Affect Disord. 93:105115.

Sakowitz, O.W., Schürmann, M., Başar, E., 2000. Oscillatory frontal theta responses are increased upon bisensory stimulation. Clin Neurophysiol. 111:884-893.

Salisbury, D.F., Shenton, M.E., McCarley, R.W., 1999. P300 topography differs in schizophrenia and manic psychosis. Biol Psychiatry. 45:98-106.

Salisbury, D.F., Shenton, M.E., Sherwood, A.R., Fischer, I.A., Yurgelun-Todd, D.A., 
Tohen, M., McCarley, R.W., 1998. Firstepisode schizophrenic psychosis differs from first-episode affective psychosis and controls in P300 amplitude over left temporal lobe. Arch Gen Psychiatry. 55:173-180.

Sarnthein, J., Petsche, H., Rappelsberger, P., Shaw, G.L., von Stein, A., 1998. Synchronization between prefrontal and posterior association cortex during human working memory. Proc Natl Acad Sci USA. 95:7092-7096.

Schulze, K.K., Hall, M.H., McDonald, C., Marshall, N., Walshe, M., Murray, R.M., Bramon, E., 2007. P50 Auditory Evoked Potential Suppression in Bipolar Unaffected Relatives. Biol Psychiatry. 62:121-128.

Schulze, K.K., Hall, M.H., McDonald, C., Marshall, N., Walshe, M., Murray, R.M., Bramon, E., 2008. Auditory P300 in patients with bipolar disorder and their unaffected relatives. Bipolar Disord. 10:377-386.

Souza, V.B., Muir, W.J., Walker, M.T., Glabus, M.F., Roxborough, H.M., Sharp, C.W., Dunan, J.R., Blackwood, D.H., 1995. Auditory P300 event-related potentials and neuropsychological performance in schizophrenia and bipolar affective disorder. Biol Psychiatry. 37:300-310.

Strakowski, S.M., DelBello, M.P., Adler, C.M., 2005. The functional neuroanatomy of bipolar disorder: a review of neuroimaging findings. Mol Psychiatry. 10:105-116.

Strik, W.K., Fallgatter, A.J., Brandeis, D., Pascual-Marqui, R.D., 1998. Threedimensional tomography of event-related potentials during response inhibition: evidence for phasic frontal lobe activation. Electroencephalogr Clin Neurophysiol. 108:406-413.

von Stein, A., Sarnthein, J., 2000. Different frequencies for different scales of cortical integration: from local gamma to long range alpha/theta synchronization. Int $\mathbf{J}$ Psychophysiol. 38:301-313.

Yener, G., Başar, E., 2010. Sensory evoked and event related oscillations in Alzheimer's disease: a short review. Cogn Neurodyn. 4, 263-274.

Yener, G.G., Güntekin, B., Oniz, A., Başar, E., 2007. Increased frontal phase-locking of event-related theta oscillations in Alzheimer patients treated with cholinesterase inhibitors. Int J Psychophysiol. 64:46-52.

Young, R.C., Biggs, J.T., Ziegler, V.E., Meyer, D.A., 1978 A rating scale for mania: reliability, validity and sensitivity. $\mathrm{Br} \mathrm{J}$ Psychiatry. 133:429-35.

Zatorre, R.J., 2001. Neural specializations for tonal processing. Ann N Y Acad Sci. 930:193-210. 


\section{CHAPTER 3}

\section{Decrease of Event-Related Delta Oscillations in Euthymic Patients with Bipolar Disorder}

Murat İlhan Atagün, Bahar Güntekin, Belinda Maşalı, Elif Tülay, Erol Başar

Psychiatry Research: Neuroimaging 2014; 223: 43-48. 


\begin{abstract}
Decreased delta oscillation upon cognitive load is common in patients with Alzheimer's disease, mild cognitive impairment, and schizophrenia (Başar and Güntekin, 2013). However, there is no previous study analyzing the delta responses in euthymic medication-free patients with bipolar disorder. Twenty-two DSM-IV diagnosed euthymic medication-free patients with bipolar disorder and twenty-one healthy controls who were matched for sex, age, and education, were enrolled in the study. EEG was recorded at 30 electrode sites using an application of an auditory oddball paradigm. The maximum peak-to-peak amplitudes for each subject's averaged delta response $(0.5-3.5 \mathrm{~Hz})$ were measured. There was a significant inter-group difference in evoked and event-related delta $(0.5-3.5 \mathrm{~Hz})$ responses $(\mathrm{p}=0.002)$. Post-hoc comparisons revealed that event-related delta oscillatory responses of the bipolar patient group were significantly lower than the healthy control group over the temporoparietal and occipital electrode sites. Euthymic bipolar patients showed reduced event-related delta oscillatory responses in comparison to healthy subjects upon cognitive load. Decrease of delta oscillations can be a common phenomenon that can be observed in different neuropsychiatric disorders with cognitive dysfunction.
\end{abstract}

Key Words: Bipolar Disorder, EEG, Delta Oscillations, Event-Related Potentials, Auditory 


\section{Introduction}

Although bipolar disorder (BD) is characterized by manic and depressive episodes, patients also suffer from cognitive dysfunctions that cannot be explained only by mood episodes (Bora et al., 2009). Cognitive task-based neuroimaging methods including electroencephalography (EEG) may provide valuable information in understanding the pathophysiology of neuropsychiatric diseases. The EEG is a cheap, noninvasive method with high temporal resolution that may provide a unique opportunity to observe cognitive processes over longer periods of time. The hemodynamic response is an indirect indicator of neuronal activity; whereas EEG can measure the bioelectrical activity of neuron populations. Poor spatial resolution is the major disadvantage of the EEG.

Some studies of the event-related potential in bipolar disorder have identified reduced P300 amplitudes (Muir et al., 1991; Salisbury et al., 1998, 1999; El-Badri et al., 2001; O’Donnell et al., 2004a, 2004b; Fridberg et al., 2009), whereas other studies reported no difference between healthy controls and patients with bipolar disorder (Souza et al., 1995; Strik et al., 1998; Hall et al., 2007; Kaya et al., 2007; Schulze et al., 2007, 2008). Furthermore, three studies reported prolonged P300 latency (O’Donnell et al., 2004; Turetsky et al., 2007; Schulze et al., 2008), whereas Salisbury et al. (1999) did not detect any delay in P300 latency. These divergent results may be related to the variable nature of bipolar disorder. Many confounders (clinical state, history of psychotic episodes, family history, medication status, etc.) may have influenced the study results.

The global P300 activity of the brain is the superimposition of multiple oscillations in delta $(0.5-3.5 \mathrm{~Hz})$, theta $(4-7 \mathrm{~Hz})$, alpha $(8-13 \mathrm{~Hz})$, beta $(18-30 \mathrm{~Hz})$ and gamma $(30-70 \mathrm{~Hz})$ frequencies, which are selectively distributed in various parts of the brain (Başar, 1998). Delta oscillations are the major component of the P300 responses (Stampfer and Başar, 1985). All brain functions are controlled by the complex integration of various parts of the brain via these oscillatory activities (Başar et al., 2001). Disturbed sensory or cognitive processing might have reflections in various frequency responses and connectivity deficits between involved brain regions may influence a certain frequency response (Başar, 2006).

Oscillatory brain responses are widely studied in schizophrenia and dementias, but studies of bipolar disorder are less common (Başar and Güntekin, 2008; Başar et al., 2012). Most consistent findings regarding bipolar disorder are obtained via auditory paradigms. For example, three studies reported decreased evoked power upon auditory steady state stimulation 
(O’Donnell et al., 2004a; Spencer et al., 2008; Oda et al., 2012). Euthymic patients with bipolar disorder showed reduced mean trial power and phase-locking factor (PLF) upon auditory steady-state stimulation (Rass et al., 2010). Medication-free euthymic patients with bipolar disorder showed decreased amplitude in slow $(4-6 \mathrm{~Hz})$ and fast $(6-8 \mathrm{~Hz})$ theta frequency bands upon application of an auditory oddball paradigm (Atagün et al., 2013). Reite et al. (2009) detected abnormal lateralization of auditory cortices in auditory steady state responses in euthymia. Furthermore, the authors indicated that medicated patients showed decreased PLF in comparison to medication-free subjects. With an auditory dual-click paradigm, Hall et al. (2011) showed that the power of the gamma response was reduced in euthymic and depressed patients with bipolar disorder. Compared to healthy subjects or patients with schizophrenia, euthymic patients with bipolar disorder had higher $(20-45 \mathrm{~Hz})$ responses to speech sounds (Oribe et al., 2010). Another recent study reported significant differences between schizophrenia and bipolar disorder in an auditory oddball paradigm (Ethridge et al., 2012). The authors indicated that N200 responses to target stimuli differentiated patients with bipolar disorder and schizophrenia from healthy subjects; late beta response power to auditory target and standard stimuli differentiated bipolar patients from schizophrenia and healthy controls. Hamm et al. (2012) showed that N100 differentiated the group with schizophrenia from bipolar and healthy control groups; and late beta responses differentiated the bipolar patient group from schizophrenia and healthy control groups.

To our knowledge, only one study reported that 'medicated' euthymic patients with bipolar disorder $(\mathrm{n}=10)$ had increased delta $(2-4 \mathrm{~Hz})$ synchronization at frontal sites (Chen et al., 2008). Many excellent pharmaco-EEG studies have examined the effects of medications on brain bioelectrical activity. Our research group previously showed that oscillatory responses can be influenced by mood stabilizers such as valproate (Özerdem et al., 2008). It has also been shown that cholinergic treatment affects oscillatory brain responses in patients with Alzheimer's disease (Yener et al., 2007). This study examines oscillatory delta responses in medication-free euthymic patients with bipolar disorder. It is hypothesized that, upon application of auditory simple and oddball paradigms, oscillatory delta responses might be altered in patients with bipolar disorder. 


\section{Methods}

\section{a. Subjects}

The study enrolled 22 euthymic, medication-free patients with bipolar disorder (19 Bipolar I, 3 Bipolar II disorder) and 21 healthy control participants matched for age, education and gender (Table 1). Diagnoses were checked by the Structured Clinical Interview (SCID) (First et al., 1996); and clinical evaluation tools for bipolar patients were Young Mania Rating Scale (YMRS) (Young et al., 1978), and Hamilton Depression Rating Scale (HAM-D) (Hamilton, 1960). Inclusion criteria for the patient group were: to have been diagnosed with bipolar disorder, euthymic for at least eight weeks and unmedicated for at least two weeks. Good medical health, as confirmed by a physical examination and routine laboratory tests, was required for participation. Exclusion criteria were: pregnancy, lactation, consumption of alcohol or substances two weeks prior to the recordings, axis I and II psychiatric co-morbidity, and neurological conditions such as neurodegenerative diseases, epilepsy, or brain surgery. Volunteers who proved to have no present or past psychiatric condition on the SCID-I interview and were reported as medically healthy on physical examination were enrolled in the control group. All participants were right-handed. All subjects were asked to avoid sleep deprivation before the experiments. All the experiments were done at the same time of day (1 pm to $5 \mathrm{pm}$ ). The study design was reviewed and approved by the ethical committee and an appropriate informed consent of the participants was obtained after the nature of the procedures had been clearly explained.

\section{b. Stimuli and procedures}

The tests were conducted in a dimly-lit isolated room. Two types of auditory stimuli-simple and oddball paradigm — were presented to the subjects via two loud-speakers positioned $50 \mathrm{~cm}$ in front of the subject. The auditory stimuli had $1000 \mathrm{~ms}$ duration. One type of stimulus $(80 \mathrm{~dB}$, $1600 \mathrm{~Hz}$ tone) was presented in the simple stimuli and no instructions were given to the subjects. Subsequently, the classical auditory oddball paradigm comprised 40 task-relevant target tones $(80 \mathrm{~dB}, 1600 \mathrm{~Hz})$ and 80 task-irrelevant non-target $(80 \mathrm{~dB}, 1500 \mathrm{~Hz})$ stimuli were presented in a random sequence. The interval between tones varied randomly between three and seven seconds. Subjects were asked to discriminate and mentally count the number of target stimuli.

\section{c. EEG recording}

An elastic cap (easy-cap) was used, containing $30 \mathrm{Ag}-\mathrm{AgCl}$ electrodes according to the 
international 10-20 system. Two linked earlobe electrodes $\left(A_{1}-A_{2}\right)$ were used for references and another pair for electrooculography (EOG) were placed on the medial upper and lateral orbital rim of the right eye. All electrode impedances were less than $10 \mathrm{k} \Omega$. The EEG was amplified by means of a BrainAmp 32-channel DC device with band limits of $0.01-250 \mathrm{~Hz}$. The EEG was digitized on-line at a sampling rate of $500 \mathrm{~Hz}$.

\section{d. Data analyses}

Artifacts in the EOG recordings were eliminated by manual off-line selective averaging. The epochs (between -500 and $1000 \mathrm{~ms}$ ) of each subject were averaged and then digital Fast Fourier Transform (FFT) based power spectrum analysis was performed (Figure 1; 10\% Hanning windowing function) in order to calculate the oscillatory delta frequency peak.

Figure 1. Power spectra of response upon auditory simple and oddball stimuli. ( $\mathrm{C}_{4}$ location) Black lines represent healthy controls; red lines represent patients with bipolar disorder.

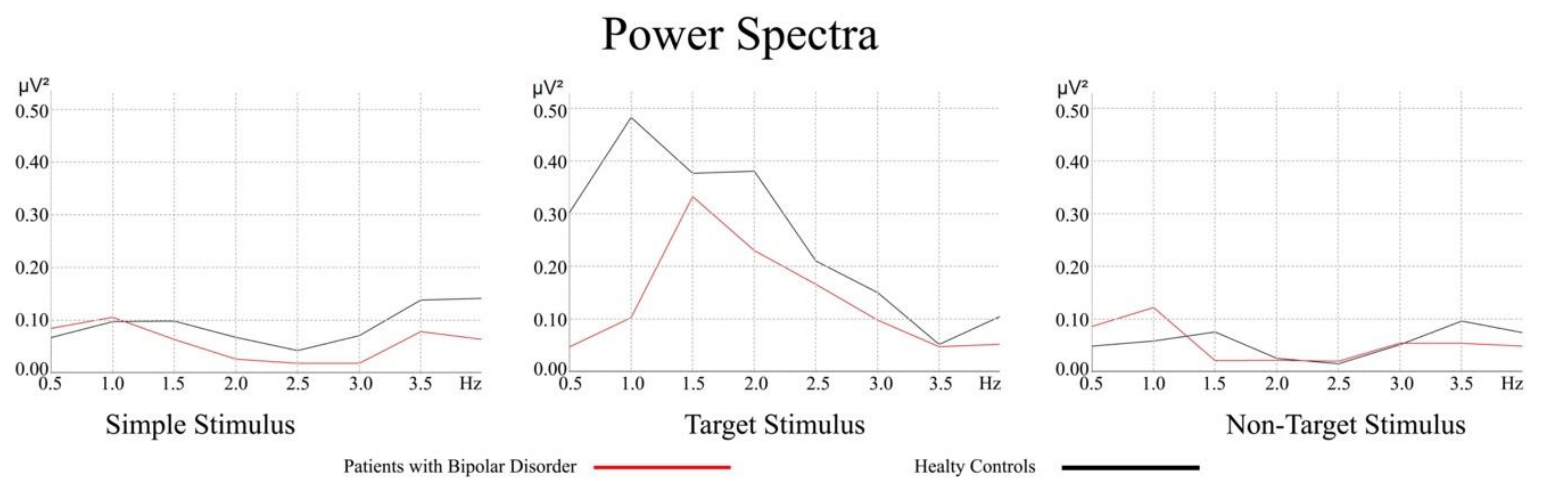

Subject averages and grand averages were calculated for each electrode site and experimental condition. Averaged evoked and event-related potentials were filtered in $0.5-3.5 \mathrm{~Hz}$ ranges. The event-related oscillatory delta responses to the target auditory stimuli were analyzed and compared. The maximum peak-to-peak amplitudes of oscillatory delta responses were calculated. Delta frequency is a slow frequency that lasts for 400 to 500 milliseconds; therefore the window was determined as 0-600 milliseconds.

\section{e. Statistical analyses}

Statistical analyses were performed by Statistica Software. Repeated measures of ANOVA were used to identify significant group differences in oscillatory delta responses for different conditions, locations and between the bipolar patient and healthy control groups. Repeated 
measures ANOVA included the bipolar patient and the healthy control groups as the betweensubject factor. Stimulus types (simple, target and non-target) at three levels, locations [frontal (F3-F4), central (C3-C4), temporal (T7-T8), temporo-parietal (Tp7-Tp8), Parietal (P3-P4) and Occipital $(\mathrm{O} 1-\mathrm{O} 2)]$ at six sites and two hemispheres (left-right) were included as withinsubject factors. Greenhouse-Geisser corrected p-values were reported. Bonferroni test was used for post-hoc comparisons. Planned comparisons were performed to analyze the differences between groups for six different locations upon application of target stimulation; it was also aimed to find the most effected electrode site. Bonferroni corrections were also performed while comparing locations between different groups during target stimulation. The number of errors by each subject was noted and, due to extreme values, logarithmic transformation was applied; the log-transformed numbers of errors are compared via the t-test. Subjects were grouped as error-positive or error-negative and compared via the chi-square test. Spearman's correlation test was conducted for each group to detect any correlation between clinical and behavioral parameters and mean amplitude values in evoked and event-related responses.

\section{Results}

Groups were age, gender, and education matched (Table 1).

Table 1. Sociodemographic and clinical characteristics of the groups.

\begin{tabular}{|c|c|c|c|c|}
\hline & & $\begin{array}{l}\text { Patients with Bipolar } \\
\text { Disorder }(n=22)\end{array}$ & $\begin{array}{l}\text { Healthy Controls } \\
\qquad(\mathrm{n}=21)\end{array}$ & $\mathrm{p}$ \\
\hline \multicolumn{2}{|l|}{ Age } & $30.82 \pm 6.46$ & $29.10 \pm 7.87$ & $0.436^{\mathrm{a}}$ \\
\hline \multicolumn{2}{|l|}{ Education } & $11.77 \pm 3.61$ & $13.41 \pm 2.69$ & $0.097^{\mathrm{a}}$ \\
\hline \multicolumn{2}{|c|}{ Gender (f/m) } & $16 / 6$ & $16 / 5$ & $0.795^{\mathrm{b}}$ \\
\hline \multicolumn{2}{|c|}{ Age at disease onset } & $21.86 \pm 6.30$ & & \\
\hline \multicolumn{2}{|c|}{ Duration of euthymia } & $48.64 \pm 37.71$ & & \\
\hline \multicolumn{2}{|c|}{ Duration of disorder } & $10.05 \pm 4.96$ & & \\
\hline \multirow{3}{*}{$\begin{array}{l}\text { Total } \\
\text { number of }\end{array}$} & Episodes & $3.91 \pm 3.13$ & & \\
\hline & Mania & $1.77 \pm 1.54$ & & \\
\hline & Depression & $1.14 \pm 1.08$ & & \\
\hline
\end{tabular}


There was a significant inter-group difference in evoked and event-related delta $(0.5-3.5 \mathrm{~Hz})$ responses $(\mathrm{p}=0.002)$. Post-hoc comparisons revealed that event-related delta oscillatory responses of the bipolar patient group were significantly lower than the healthy control group over the temporoparietal and occipital electrode sites. Repeated measures ANOVA revealed a significant stimulus type interaction $[\mathrm{F}(2,82): 63.26, \mathrm{p}<0.001]$. Post-hoc comparisons showed that oscillatory delta responses upon auditory target stimuli $(8.71 \pm 3.99)$ were significantly higher than both simple stimuli $(4.93 \pm 2.19)(\mathrm{p}<0.00001)$ and non-target stimuli $(4.78 \pm 2.26)$ $(p<0.00001)$. ANOVA revealed significant results for location $[F(5,205)=50.86, p<0.001]$. Post-hoc comparisons showed that amplitudes of frontal, central and parietal locations were significantly higher than amplitudes of temporal, temporo-parietal and occipital locations. ANOVA revealed significant results for [stimulus $\times$ location] $[F(5,205): 11.51, p<0.001]$ interaction. Post-hoc comparisons showed that the difference between target stimulation versus simple stimuli $(\mathrm{p}<0.00001)$ and versus non-target stimuli $(\mathrm{p}<0.00001)$ were most prominent at parietal location.

The ANOVA revealed [stimulus $\times$ laterality] difference $[\mathrm{F}(2,82)=4.36, \mathrm{p}=0.016]$. Post-hoc comparisons showed that amplitude values of right hemispheric responses upon auditory target stimuli were significantly greater than left hemispheric responses $(\mathrm{p}=0.002)$.

Delta oscillatory responses differed significantly between the bipolar patient and healthy control groups $[\mathrm{F}(1,41)=11.17, \mathrm{p}=0.002]$. The stimulusXgroupXlocation or groupXlocation interactions were not significant thus showing that this difference between groups were found in overall electrode sites. However, we have also observed that this difference could be more prominent in some of the electrodes, especially upon target stimulation. Accordingly we have run planned comparisons in which we have analyzed delta oscillatory responses only for target stimulation and for frontal, central, temporal, temporo-parietal and occipital locations. After Bonferroni correction the significance level for $p$ value was determined as $p=0,008$. The planned comparisons showed that healthy subjects had higher delta responses than bipolar disorder patients over central $(\mathrm{p}=0.04)$, temporal $(\mathrm{p}=0.01)$, temporo-pariteal $(\mathrm{p}=0.006)$, parietal $(\mathrm{p}=0.03)$ and occipital $(\mathrm{p}=0.008)$ locations. After Bonferroni correction, it was seen that healthy subjects had higher delta responses than bipolar patients only for temporo-parietal $(p=0.006)$, and occipital $(\mathrm{p}=0.008)$ locations upon target stimulation. Differences between groups are presented in Figure 2. 
Figure 2. Event-related delta $(0.5-3.5 \mathrm{~Hz})$ oscillatory responses upon auditory target stimuli.

Red lines represent patients with bipolar disorder and green bars represent healthy subjects. Statistically significant differences are marked with * sign (for comparison of temporoparietal location $\mathrm{p}=0.006$, for occipital location $\mathrm{p}=0.008$ ).

\section{Event Related Delta Responses}

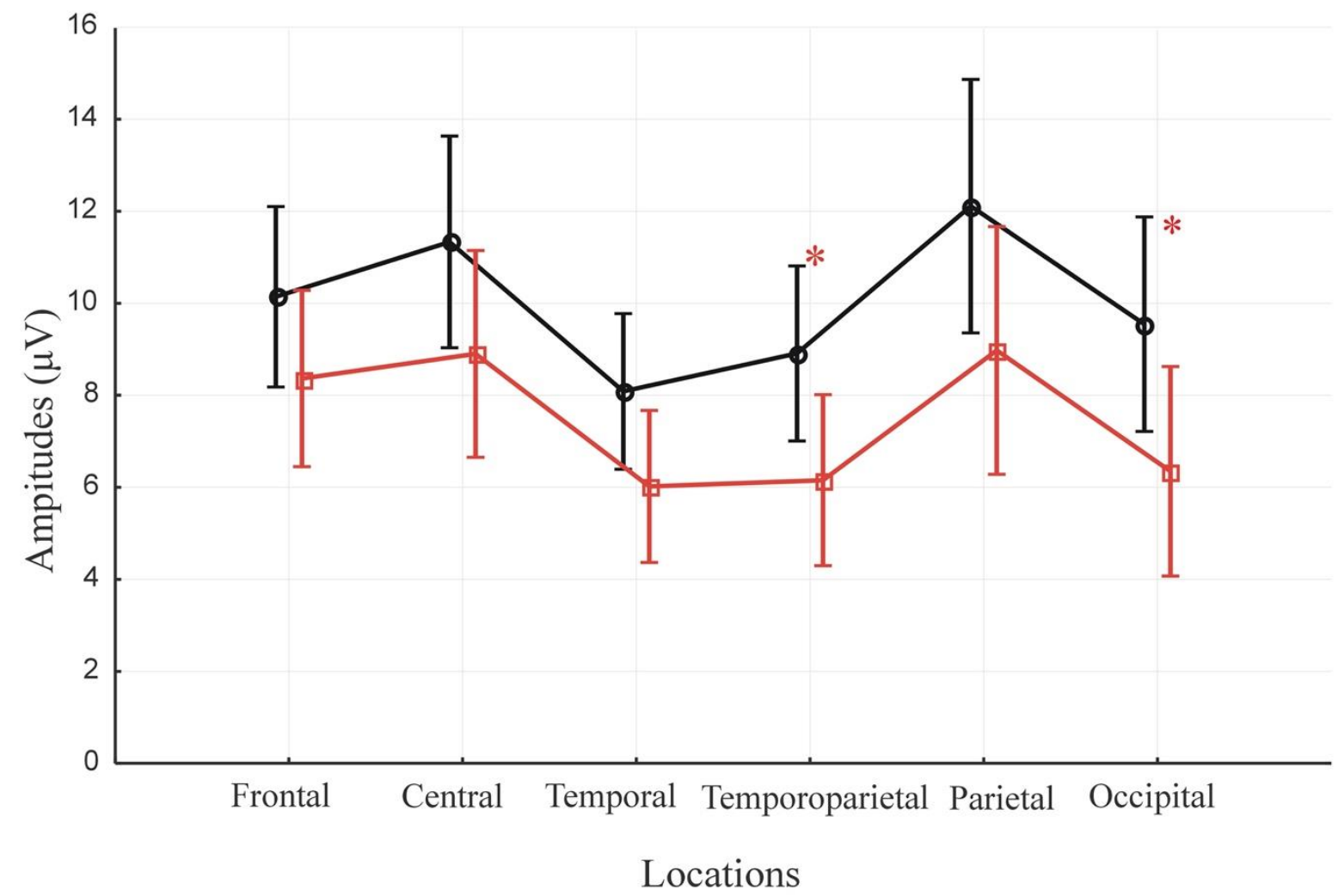

Analysis of behavioral data showed that 18 out of 22 patients with bipolar disorder made errors, whereas 9 out of 21 healthy controls made errors $\left(\mathrm{p}=0.013, \chi^{2}=6.19\right)$. The number of errors (log transformed) differed significantly between groups $(\mathrm{p}=0.004, \mathrm{Z}=-2.85)$. No correlation was identified between the number of errors and the amplitudes of target responses.

Correlation analysis showed that age and age at disease onset significantly correlated with the amplitude of target responses in the bipolar group (Table 2). Young Mania Rating Scale scores correlated with amplitude at the right temporo-parietal $(\mathrm{p}<0.05, \mathrm{r}=0.45)$ and occipital $(\mathrm{p}<0.05$, $\mathrm{r}=0.45$ ) locations. Education negatively correlated with the number of errors in target detection $(\mathrm{p}<0.05, \mathrm{r}=-0.48)$. Duration of disease correlated with number of errors in target detection $(\mathrm{p}<0.05, \mathrm{r}=0.51)$. Other variables (education, HAM-D scores, duration of disease, duration of euthymia and number of total/manic/depressive episodes) did not show any correlation with 
mean amplitude values. However, these significant correlations were not significant after Bonferroni correction.

Table 2. Correlations in the bipolar disorder group

\begin{tabular}{|c|c|c|c|c|c|c|c|c|c|c|c|c|c|}
\hline & $\begin{array}{l}\text { Type of } \\
\text { Stimuli }\end{array}$ & $\mathbf{F}_{3}$ & $\mathbf{F}_{4}$ & $\mathrm{C}_{3}$ & $\mathrm{C}_{4}$ & $\mathbf{T}_{7}$ & $\mathbf{T}_{8}$ & $\mathbf{T} \mathbf{p}_{7}$ & Tps & $\mathbf{P}_{3}$ & $\mathbf{P}_{4}$ & $\mathbf{O}_{1}$ & $\mathbf{O}_{2}$ \\
\hline \multirow{2}{*}{ Age } & Simple & -0.31 & -0.25 & -0.42 & $-0.42^{*}$ & -0.33 & -0.36 & -0.12 & -0.22 & $-0.46^{*}$ & -0.39 & -0.14 & -0.25 \\
\hline & Target & -0.33 & -0.12 & $-0.49^{*}$ & $-0.49^{*}$ & -0.33 & $-0.50^{*}$ & -0.38 & $-0.56^{\ddagger}$ & $-0.45^{*}$ & $-0.47^{*}$ & -0.41 & $-0.48^{*}$ \\
\hline YMRS & target & 0.16 & 0.03 & 0.14 & 0.14 & 0.16 & 0.21 & 0.29 & $0.45^{*}$ & 0.26 & 0.28 & 0.32 & $0.45^{*}$ \\
\hline $\begin{array}{l}\text { Age of } \\
\text { Onset }\end{array}$ & target & -0.35 & -0.27 & $-0.45^{*}$ & $-0.47^{*}$ & $-0.45^{*}$ & -0.30 & $-0.43^{*}$ & -0.36 & -0.41 & $-0.43^{*}$ & -0.21 & -0.34 \\
\hline
\end{tabular}

Spearman's Rho Correlation Test, $r$ values. ${ }^{*} p<0.05,{ }^{*} p<0.01$. YMRS: Young Mania Rating Scale. Education, Hamilton depression rating scale score, duration of disease, duration of euthymia and number of (total/manic/depressive) episodes did not show any correlation with amplitudes of delta oscillations.

\section{Discussion}

The findings show that evoked and event-related delta oscillations in patients with bipolar disorder are significantly lower than in healthy controls at central, parietal, temporal, temporoparietal and occipital regions. Oscillatory delta responses upon cognitive tasks have also been reported to be significantly decreased in different groups such as schizophrenia (Ergen et al., 2008; Ford et al., 2008; Bates et al., 2009; Doege et al., 2010), Alzheimer's disease (Güntekin et al., 2008; Yener et al., 2008; Başar et al., 2010; Yener et al., 2012) and elderly healthy subjects (Schmiedt-Fehr and Başar-Eroğlu, 2011). This suggests that delta oscillations might play a critical role in cognition, which might be commonly influenced by neuropathology of various neuropsychiatric disorders, as proposed by Başar and Güntekin (2013).

Delta oscillations are related to focused attention, signal detection, recognition, and decisionmaking (Başar-Eroğlu et al., 1992; Başar et al., 1998, 1999, 2001; Schürmann et al., 2001). Additionally, delta oscillations are also involved in cortical communication over long distances (Bruns and Eckhorn, 2004). Yener et al. (2010, 2012) suggested there were two different networks activated in delta frequency in response to different stimulus modalities, according to the stimulation characteristics and requirements (i.e. sensory or cognitive demands). Although the groups differed in event-related delta oscillations, there was no difference between healthy controls and patients with Alzheimer's disease in their evoked responses (Yener et al. 2012). 
Evoked oscillations do not include cognition related components and event-related oscillations 䍃 include cognitive task related responses.

Independent of the group ( $n=43$ ), event-related delta oscillations were higher in frontal, central and parietal locations compared with temporal and occipital locations. This pattern may indicate that operations with delta oscillations spread to fronto-parietal regions upon cognitive demands of the oddball paradigm (Schürmann et al., 1995; Başar, 1998, 1999; Ishii et al., 2009; Mathes et al., 2012). However, despite the general trend, the between-group difference in delta oscillations in the oddball paradigm was not significant at frontal locations.

Dysfunction of certain networks might have several sub-cellular, cellular or tissue level reasons (such as synaptic transmission, axonal transfer, myelination), that may cause disrupted connectivity (Tkachev et al., 2003; Uranova et al., 2004), and therefore synchronization deficits in various neuropsychiatric diseases (Güntekin et al., 2008; Özerdem et al., 2010, 2011; Schmiedt-Fehr and Basar-Eroglu, 2011; Başar-Eroğlu et al., 2008; Başar and Güntekin, 2008; Uhlhaas et al., 2011). However, although these neuropsychiatric diseases have common characteristics in their physiopathology, the spatial distribution and temporal dynamics of connectivity deficits may determine the clinical course of the disorder.

The major limitation of the present study is the relatively small sample size. The number of female subjects in the groups was higher than male participants ( $n=43$, female/male: $32 / 11$ ). The study included three patients with bipolar disorder II, and it is still not clear whether there is a difference between bipolar I and II disorders in terms of oscillatory brain responses. Most of the patients were mild cases and therefore they were off-medications. On the other hand, medication-free patients can be seen as the major strength of the study, because of providing the chance to understand de novo neurophysiology of bipolar disorder. In addition, all patients were euthymic and none of the patients had sub-threshold depressive or manic symptoms.

\section{Conclusion}

It can be suggested that delta oscillations are critically important in cognitive functions, and that diminished oscillatory delta responses may be a common characteristic of cognitive dysfunction in neuropsychiatric diseases (Başar and Güntekin, 2013; Başar, 2011). 
Table 3. Brain oscillations studies by our group in medication-free patients with bipolar disorder.

\begin{tabular}{|l|l|l|}
\hline $\begin{array}{c}\text { Study } \\
\text { Chronological } \\
\text { Order })\end{array}$ & $\begin{array}{c}\text { Clinical } \\
\text { State }\end{array}$ & \multicolumn{1}{c|}{ Major Findings } \\
\hline Özerdem et al., 2008 & Mania & $\begin{array}{l}\text { Increased visual event-related beta (18-30 Hz) and reduced event-related } \\
\text { alpha (8-13 Hz) responses }\end{array}$ \\
\hline Özerdem et al., 2010 & Mania & Reduced gamma (28-48 Hz) coherence \\
\hline Özerdem et al., 2011 & Euthymia & Reduced gamma (28-48 Hz) coherence \\
\hline Başar et al., 2012 & Euthymia & $\begin{array}{l}\text { Decreased spontaneous alpha (8-13 Hz) activity and visual-evoked alpha } \\
(8-13 \mathrm{~Hz}) \text { responses }\end{array}$ \\
\hline Atagün et al., 2013 & Euthymia & $\begin{array}{l}\text { Decreased evoked and event-related slow (4-6 Hz) theta and decreased } \\
\text { event-related fast (6-8 Hz) theta responses. Fast theta }(6-8 \mathrm{~Hz}) \text { was } \\
\text { more responsive to cognitive components of the task. }\end{array}$ \\
\hline This study & Euthymia & $\begin{array}{l}\text { Decreased auditory-evoked and event-related delta }(0.5-3.5 \mathrm{~Hz}) \\
\text { responses }\end{array}$ \\
\hline
\end{tabular}

Abnormalities of brain oscillations may have specific profiles in neuropsychiatric disorders and those profiles may potentially discriminate disorders. These profiles can only be detected by studies that have eliminated confounders like medications. As summarized in Table 3, to date we have detected increased event-related beta and decreased event-related alpha responses in mania (Özerdem et al., 2008), reduced gamma coherence in mania (Özerdem et al., 2010) and euthymia (Özerdem et al., 2011), decreased spontaneous EEG alpha activity and evoked visual alpha response in euthymia (Başar et al. 2012), decreased evoked and event-related slow theta (4-6 Hz) and event-related fast theta $(6-8 \mathrm{~Hz})$ responses in euthymia (Atagün et al., 2013), and the present study reveals that auditory-evoked and event-related delta responses are decreased in euthymic medication-free patients with bipolar disorder. Further studies should focus on brain oscillations in different clinical states for bipolar disorder. Comparing bipolar disorder to schizophrenia and schizoaffective disorder may help to identify differences and allow us to investigate whether there are any biological correlates of clinical differences between these psychotic spectrum disorders.

Acknowledgement: The authors would like to thank to E. Timuçin Oral MD for his effort to bring two institutions together for a long-term collaboration. Subjects of the study were enrolled from outpatient units of Bakırköy Research and Training Hospital for Psychiatry, Neurology and Neurosurgery, when the authors MIA and BM were working in the hospital between 2009 and 2011. 


\section{References}

Atagün, M.İ., Güntekin, B., Özerdem, A., Tülay, E., Başar, E., 2013. Decrease of theta response in euthymic bipolar patients during an oddball paradigm. Cogn Neurodyn. 7, 213-223.

Başar, E., 1998. Brain function and oscillations I: principles and approaches. Springer, Heidelberg, pp. 269.

Başar, E., 1999. Brain function and oscillations: II. Integrative brain function. Neurophysiology and cognitive processes. Springer, Heidelberg, pp.161-189.

Başar, E., 2006. The theory of whole-brainwork. Int J Psychophysiol. 60, 133-138.

Başar, E., 2011. Brain-body-mind in the Nebulous Cartesian System: A Holistic Approach by Oscillations. Springer, pp. 131.

Başar, E., Başar-Eroğlu, C., Karakaş, S., Schürmann, M., 1999. Are cognitive processes manifested in event-related gamma, alpha, theta and delta oscillations in the EEG? Neurosci Lett. 259, 165-168.

Başar, E., Güntekin. B., 2008. A review of brain oscillations in cognitive disorders and the role of neurotransmitters. Brain Res. 1235, 172-193.

Başar, E., Güntekin, B., 2013. Review of delta, theta, alpha, beta, and gamma response oscillations in neuropsychiatric disorders. Suppl Clin Neurophysiol. 62, 303-341.

Başar, E., Güntekin, B., Atagün, İ., Turp Gölbaşı, B., Tülay, E., Özerdem, A., 2012. Brain's alpha activity is highly reduced in euthymic bipolar disorder patients. Cogn Neurodyn. 6, 11-20.

Başar, E., Güntekin, B., Tülay, E., Yener, G.G., 2010. Evoked and event related coherence of Alzheimer patients manifest differentiation of sensory-cognitive networks. Brain Res. 1357, 79-90.

Başar, E., Schürmann, M., Sakowitz, O., 2001. The selectively distributed theta system: functions. Int J Psychophysiol. 39, 197-212.

Başar-Eroğlu, C., Başar, E., Demiralp, T., Schürmann, M., 1992. P300-response: possible psychophysiological correlates in delta and theta frequency channels: a review. Int J Psychophysiol. 13, 161-179.

Basar-Eroglu, C., Schmiedt-Fehr, C., Mathes, B., Zimmermann, J., Brand, A., 2009. Are oscillatory brain responses generally reduced in schizophrenia during long sustained attentional processing? Int $\mathrm{J}$ Psychophysiol. 71, 75-83.

Basar-Eroglu, C., Schmiedt-Fehr, C., Marbach, S., Brand, A., Mathes, B., 2008. Altered oscillatory alpha and theta networks in schizophrenia. Brain Res. 1235, 143-152.

Bruder, G.E., Stewart, J.W., Towey, J.P., Friedman, D., Tenke, C.E., Voglmaier, M.M., Leite, P., Cohen, P., Quitkin, F.M., 1992. Abnormal cerebral laterality in bipolar depression: convergence of behavioral and brain event-related potential findings. Biol Psychiatry. 32, 33-47.

Bates, A.T., Kiehl, K.A., Laurens, K.R., Liddle, P.F., 2009. Low-frequency EEG oscillations associated with information processing in schizophrenia. Schizophr Res. 115, 222-230.

Bora, E., Yucel, M., Pantelis, C., 2009. Cognitive endophenotypes of bipolar disorder: a meta-analysis of neuropsychological deficits in euthymic patients and their first-degree relatives. $\mathbf{J}$ Affect Disord. 113, 1-20.

Bruns, A., Eckhorn, R., 2004. Task-related coupling from high- to low-frequency 
signals among visual cortical areas in human subdural recordings. Int J Psychophysiol. 51, 97-116.

Chen, S.S., Tu, P.C., Su, T.P., Hsieh, J.C., Lin, Y.C., Chen, L.F., 2008. Impaired frontal synchronization of spontaneous magnetoencephalographic activity in patients with bipolar disorder. Neurosci Lett. $445,174-178$.

Doege, K., Jansen, M., Mallikarjun, P., Liddle, E.B., Liddle, P.F., 2010. How much does phase resetting contribute to event-related EEG abnormalities in schizophrenia? Neurosci Lett. 481, 1-5.

El-Badri, S.M., Ashton, C.H., Moore, P.B., Mursh, V.R., Ferrier, I.N., 2001. Electrophysiological and cognitive function in young euthymic patients with bipolar affective disorder. Bipolar Disord. 3, 79-87.

Ergen, M., Marbach, S., Brand, A., BaşarEroğlu, C., Demiralp, T., 2008. P3 and delta band responses in visual oddball paradigm in schizophrenia. Neurosci Lett. 440, 304-308.

Ethridge, L.E., Hamm, J.P., Shapiro, J.R., Summerfelt, A.T., Keedy, S.K., Stevens, M.C., Pearlson, G., Tamminga, C.A., Boutros, N.N., Sweeney, J.A., Keshavan, M.S., Thaker, G., Clementz, B.A., 2012. Neural activations during auditory oddball processing discriminating schizophrenia and psychotic bipolar disorder. Biol Psychiatry. 72, 766-774.

First, M.B., Gibbon, M., Spitzer, R.L., Gibbon, M., Williams, J.B.W., 1996. User's guide for the structured interview for DSM-IV axis I disorders-research version (SCID-I, version 2.0, February 1996 final version). Biometrics Research, New York.

Ford, J.M., Roach, B.J., Hoffman, R.S., Mathalon, D.H., 2008. The dependence of P300 amplitude on gamma synchrony breaks down in schizophrenia. Brain Res. 1235, 133-142.

Fridberg, D.J., Hetrick, W.P., Brenner, C.A., Shekhar A, Steffen AN, Malloy FW, O'Donnell, B.F., 2009. Relationships between auditory event-related potentials and mood state, medication, and comorbid psychiatric illness in patients with bipolar disorder. Bipolar Disord. 11, 857-866.

Güntekin, B., Saatçi, E., Yener, G., 2008. Decrease of evoked delta, theta and alpha coherences in Alzheimer patients during a visual oddball paradigm. Brain Res. 1235, 109-116.

Hall, M.H., Rijsdijk, F., Kalidindi, S., Schulze, K., Kravariti, E., Kane, F., Sham, P., Bramon, E., Murray, R.M., 2007. Genetic overlap between bipolar illness and eventrelated potentials. Psychol Med. 37, 667678.

Hamilton, M., 1960. A rating scale for depression. J Neurol, Neurosurg Psychiat. $23,56-62$.

Hamm, J.P., Ethridge, L.E,, Shapiro, J.R., Stevens, M.C., Boutros, N.N., Summerfelt, A.T., Keshavan, M.S., Sweeney, J.A., Pearlson, G., Tamminga, C.A., Thaker, G., Clementz, B.A., 2012. Spatiotemporal and frequency domain analysis of auditory paired stimuli processing in schizophrenia and bipolar disorder with psychosis. Psychophysiol. 49, 522-530.

Ishii, R., Canuet, L., Herdman, A., Gunji, A., Iwase, M., Takahashi, H., Nakahachi, T., Hirata, M., Robinson, S.E., Pantev, C., Takeda, M., 2009. Cortical oscillatory power changes during auditory oddball task revealed by spatially filtered magnetoencephalography. Clin Neurophysiol. 120, 497-504.

Kaya, E., Aydemir, O., Selçuki, D., 2007. Residual symptoms in bipolar disorder: the 
effect of the last episode after remission. Prog Neuropsychopharmacol Biol Psychiat. 31, 1387-1392.

Mathes, B., Schmiedt, J., Sschmiedt-Fehr, C., Pantelis, C., Başar-Eroğlu, C., 2012. New rather than old? For working memory tasks with abstract patterns the P3 and the singletrial delta response are larger for modified than identical probe stimuli. Psychophysiol. 49, 920-932.

Muir, W.J., St Clair, D.M., Blackwood, D.H., 1991. Long-latency auditory event-related potentials in schizophrenia and in bipolar and unipolar affective disorder. Psychol Med. 21, 867-879.

O'Donnell, B.F., Hetrick, W.P., Vohs, J.L., Krishnan, G.P., Carroll, C.A., Shekhar, A., 2004a. Neural synchronization deficits to auditory stimulation in bipolar disorder. NeuroReport 15, 1369-1372.

O’Donnell, B.F., Vohs, J.L., Hetrick, W.P., Carroll, C.A., Shekhar, A., 2004b. Auditory event-related potential abnormalities in bipolar disorder and schizophrenia. International Journal of Psychophysiol. 53, 45-55.

Oda, Y., Onitsuka, T., Tsuchimoto, R., Hirano, S., Oribe, N., Ueno, T., Hirano, Y., Nakamura, I., Miura, T., Kanba, S., 2012. Gamma band neural synchronization deficits for auditory steady state responses in bipolar disorder patients. PLoSOne 7, e39955.

Oribe, N., Onitsuka, T., Hirano, S., Hirano, Y., Maekawa, T., Obayashi, C., Ueno, T., Kasai, K., Kanba, S., 2010. Differentiation between bipolar disorder and schizophrenia revealed by neural oscillation to speech sounds: an MEG study. Bipolar Disord. 12, 804-812.

Özerdem, A., Güntekin, B., Tunca, Z., Başar, E., 2008. Brain oscillatory responses in patients with bipolar disorder manic episode before and after valproate treatment. Brain Res. 1235, 98-108.
Özerdem, A., Güntekin, B., Saatçi, E., Tunca, Z., Başar, E., 2010. Disturbance in long distance gamma coherence in bipolar disorder. Prog Neuropsychopharmacol Biol Psychiat. 34, 861-865.

Özerdem, A., Güntekin, B., Atagün, I., Turp, B., Başar, E., 2011. Reduced long distance gamma (28-48 Hz) coherence in euthymic patients with bipolar disorder. J Affect Disord. 132, 325-332.

Rass, O., Krishnan, G., Brenner, C.A., Hetrick, W.P., Merrill, C.C., Shekhar, A., O'Donnell, B.F., 2010. Auditory steady state response in bipolar disorder: relation to clinical state, cognitive performance, medication status, and substance disorders. Bipolar Disord. 12, 793-803.

Reite, M., Teale, P., Rojas, D.C., Reite, E., Asherin, R., Hernandez, O., 2009. MEG auditory evoked fields suggest altered structural/functional asymmetry in primary but not secondary auditory cortex in bipolar disorder. Bipolar Disord. 11, 371-381.

Salisbury, D.F., Shenton, M.E., Sherwood, A.R., Fischer, I.A., Yurgelun-Todd, D.A., Tohen, M., McCarley, R.W., 1998. Firstepisode schizophrenic psychosis differs from first-episode affective psychosis and controls in P300 amplitude over left temporal lobe. Arch Gen Psychiatry. 55, 173-180.

Salisbury, D.F., Shenton, M.E., McCarley, R.W., 1999. P300 topography differs in schizophrenia and manic psychosis. Biol Psychiatry. 45, 98-106.

Schmiedt-Fehr, C., Basar-Eroglu, C., 2011. Event-related delta and theta brain oscillations reflect age-related changes in both a general and a specific neuronal inhibitory mechanism. Clin Neurophysiol. 122, 1156-1167.

Schulze, K.K., Hall, M.H., McDonald, C., Marshall, N., Walshe, M., Murray, R.M., Bramon, E., 2007. P50 Auditory evoked 
potential suppression in bipolar unaffected relatives. Biol Psychiatry. 62, 121-128.

Schulze, K.K., Hall, M.H., McDonald, C., Marshall, N., Walshe, M., Murray, R.M., Bramon, E., 2008. Auditory P300 in patients with bipolar disorder and their unaffected relatives. Bipolar Disord. 10, 377-386.

Schürmann, M., Başar-Eroğlu, C., Kolev, V., Başar, E., 1995. A new metric for analyzing single-trial event-related potentials (ERPs): application to human visual P300 delta response. Neurosci Lett. 197, 167-170.

Schürmann, M., Başar-Eroğlu, C., Kolev, V., Başar, E., 2001. Delta responses and cognitive processing: single-trial evaluations of human visual P300. Int J Psychophysiol. 39, 229-239.

Souza, V.B., Muir, W.J., Walker, M.T,, Glabus, M.F., Roxborough, H.M., Sharp, C.W., Dunan, J.R., Blackwood, D.H., 1995. Auditory P300 event-related potentials and neuropsychological performance in schizophrenia and bipolar affective disorder. Biol Psychiatry. 37, 300-310.

Spencer, K.M., Salisbury, D.F., Shenton, M.E., McCarley, R.W., 2008. Gamma-band auditory steady-state responses are impaired in first episode psychosis. Biol Psychiatry. 64, 369-375.

Strik, W.K., Fallgatter, A.J., Brandeis, D., Pascual-Marqui, R.D., 1998. Threedimensional tomography of event-related potentials during response inhibition: evidence for phasic frontal lobe activation. Electroencephal Clin Neurophysiol. 108, 406-413.

Stampfer, H.G., Başar, E., 1985. Does frequency analysis lead to better understanding of human event-related potentials? Int J Neurosci 26, 181-196.

Tkachev, D., Mimmack, M.L., Ryan, M.M., Wayland, M., Freeman, T., Jones, P.B.,
Starkey, M., Webster, M.J., Yolken, R.H., Bahn, S., 2003. Oligodendrocyte dysfunction in schizophrenia and bipolar disorder. Lancet 362, 798-805.

Turetsky, B.I., Kohler, C.G., Indersmitten, T., Bhati, M.T., Charbonnier, D., Gur, R.C., 2007. Facial emotion recognition in schizophrenia: when and why does it go awry? Schizophr Res. 94, 253-263.

Uhlhaas, P.J., Singer, W., 2006. Neural synchrony in brain disorders: relevance for cognitive dysfunctions and pathophysiology. Neuron. 52, 155-168.

Uranova, N.A., Vostrikov, V.M., Orlovskaya, D.D., Rachmanova, V.I., 2004. Oligodendroglial density in the prefrontal cortex in schizophrenia and mood disorders: a study from the Stanley Neuropathology Consortium. Schizophr Res. 67, 269-275.

Yener, G., Güntekin, B., Başar, E., 2008. Eventrelated delta oscillatory responses of Alzheimer patients. Eur J Neurol. 15, 540547.

Yener, G., Başar, E., 2010. Sensory evoked and event related oscillations in Alzheimer's disease: a short review. Cogn. Neurodyn. 4, 263-274.

Yener, G.G., Güntekin, B., Oniz, A., Başar, E., 2007. Increased frontal phase-locking of event-related theta oscillations in Alzheimer patients treated with cholinesterase inhibitors. Int J Psychophysiol. 64, 46-52.

Yener, G.G., Güntekin, B., Örken, D.N., Tülay, E., Forta, H., Başar, E., 2012. Auditory delta event-related oscillatory responses are decreased in Alzheimer's disease. Behav Neurol. 25, 3-11.

Young, R.C., Biggs, J.T., Ziegler, V.E., Meyer, D.A., 1978. A rating scale for mania: reliability, validity and sensitivity. $\mathrm{Br} \mathrm{J}$ Psychiatry. 133, 429-435 


\section{CHAPTER 4}

\section{Lithium Excessively Enhances Event Related Beta Oscillations in Patients with Bipolar Disorder}

Murat İlhan Atagün, Bahar Güntekin, Devran Tan, Emine Elif Tülay, Erol Başar

Journal of Affective Disorders 2015; 170: 59-65 


\section{Abstract}

Background: Previous resting-state electroencephalography studies have consistently shown that lithium enhances delta and theta oscillations in default mode networks. Cognitive task based networks differ from resting-state networks and this is the first study to investigate effects of lithium on evoked and event-related beta oscillatory responses of patients with bipolar disorder.

Methods: The study included 16 euthymic patients with bipolar disorder on lithium monotherapy, 22 euthymic medication-free patients with bipolar disorder and 21 healthy participants. The maximum peak-to-peak amplitudes were measured for each subject's averaged beta responses $(14-28 \mathrm{~Hz})$ in the $0-300 \mathrm{~ms}$ time window. Auditory simple and oddball paradigm were presented to obtain evoked and event-related beta oscillatory responses.

Results: There were significant differences in beta oscillatory responses between groups $(\mathrm{p}=0.010)$. Repeated measures of ANOVA revealed location $(\mathrm{p}=0.007)$, lateralityXgroup $(p=0.043)$ and stimulusXlocation $(p=0.013)$ type effects. Serum lithium levels were correlated with beta responses.

Limitations: The lithium group had higher number of previous episodes, suggesting that patients of the lithium were more severe cases than patients of the medication-free group.

Discussion: Lithium stimulates neuroplastic cascades and beta oscillations become prominent during neuroplastic changes. Excessively enhanced beta oscillatory responses in the lithium-treated patients may be indicative of excessive activation of the neuron groups of the certain cognitive networks and dysfunctional GABAergic modulation during cognitive activity.

Key Words: Bipolar Disorder, Brain Oscillations, Event-Related Oscillations, Lithium, Euthymia 


\section{Introduction}

Lithium has several neurotrophic and neuroplastic effects that may trigger long-term changes on synaptic plasticity and networking functions (Kim and Thayer, 2009). Increasing volume and density of gray matter (Bearden et al., 2007; Moore et al., 2000a), improving white matter connectivity (Benedetti et al., 2013) and increasing brain levels of $\mathrm{N}$-acetyl-aspartate, a marker of neuronal function and viability (Moore et al., 2000b) are some instances of lithium's beneficial effects on neuronal integrity.

Electroencephalography (EEG) is an electrophysiological method with a high temporal resolution that may provide valuable advantages for research areas that focus on cognitive networks (Başar, 2010). Either cognitive or sensory, all brain activities are operated in a specific oscillatory activity, thus all brain activities are governed by specific brain oscillations (Başar et al., 1998; Başar, 1999; Başar et al., 2001). Although dysfunction in sensory or cognitive processes cannot be explained by a frequency, connectivity characteristics may cause differences in a frequency response (Başar, 2006). Within the last two decades, brain oscillatory analyses have been applied to clinical pathologies, including bipolar disorder (Başar et al., 2013; Başar and Güntekin, 2008; Basar et al., 2013).

Despite the wealth of electroencephalography and magnetoencephalography studies in bipolar disorder, very limited studies reported beta frequency abnormalities (O’Donnell et al., 2004; Rass et al., 2010; Özerdem et al., 2008; Hamm et al., 2012; Ethridge et al., 2012; Lee et al., 2010). Patients with bipolar disorder in either manic or mixed mood state showed deficits in responses of beta and gamma frequency ranges upon auditory steady-state stimulation (O’Donnell et al., 2004; Rass et al., 2010). Manic patients showed increased beta and alpha oscillations upon a visual oddball paradigm, and treatment of the episode with valproate reduced the responses (Özerdem et al., 2008). Late beta response power to auditory target and standard stimuli was increased in bipolar disorder group and this finding differentiated psychotic bipolar patients from schizophrenia and healthy control groups (Hamm et al., 2012; Ethridge et al., 2012). Ethridge and colleagues (2012) also reported that depression scores were correlated with the beta frequency increase in bipolar disorder group, which suggest a relationship between emotional dysregulation and increased beta responses in the bipolar disorder group. Similarly, in comparison to major depression or healthy control groups, bipolar disorder group had increased alpha and beta oscillatory responses upon an emotional task (Lee et al., 2010). Most of these studies had mixed samples and medicated patients. However, both medications and clinical state may substantially alter neurophysiology, particularly beta 
oscillations are more vulnerable to neurochemical changes in particular Gamma-AminoButyric-Acid (GABA) and n-Methyl-d-Aspartate (NMDA) receptor activities (Arai and Natsume, 2006; Traub et al., 2004).

Previous studies by our group with medication-free patients with bipolar disorder also showed that in euthymia evoked and event related theta (Atagün et al., 2013b) and delta oscillations (Atagün et al., 2014) decreased upon auditory oddball paradigm. Resting state alpha activity and evoked alpha responses decreased (Başar et al., 2012). Gamma coherence is reduced in mania (Özerdem et al., 2010) and euthymia (Özerdem et al., 2011). It can be concluded that medication-free patients with bipolar disorder have a reduction in neural activation energy and reduced coherence values, which may mean a loss of functional connectivity and activation deficits.

The most consistent finding of previous lithium resting state EEG studies is increased power of resting state delta and theta activity in patients with bipolar disorder (Schulz et al., 2000; Zakowska-Dabrowska and Rybakowski, 1973; Hyun et al., 2011) or in healthy volunteers (Karniol et al., 1978; Thau et al., 1988). Ulrich and colleagues (Ulrich et al., 1987; Ulrich et al., 1990) detected enhanced alpha power in healthy male volunteers. Most of the abovementioned lithium-EEG studies were with mixed samples; patients were also taking various additional psychotropic medications.

However, it has recently been shown that task based networks differ from default-mode networks (Moussa et al.,2012; Kirschner et al., 2012). Accordingly, cognitive tasks may trigger activities that require the brain to reorganize and establish distinct networks with distinct characteristics. For instance, fast frequencies are related to cognitive activity in local networks (Başar et al., 2013), whereas slow frequencies -either in rest and activity- are related with activities of longer distances and larger amounts of neurons (Bruns and Eckhorn, 2004). There has not yet been a study that evaluates whether lithium has any different effect on cognition based networks. The effects of external manipulations (like medications) may become observable in responses to stimuli of either a bottom-up or a top-down task (Kenemans and Kähkönen, 2011).

Although it needs to be translated into human neuroscience, neuroplasticity drives networks to oscillate with beta frequency, instead of gamma frequency in animal studies (Whittington et al., 2000). As mood stabilizers induce neuroplastic cascades (Kim and Thayer, 2009; Soiero-deSouza et al., 2012), and since beta oscillations are related to neuroplastic changes (Whittington et al., 2000), it could be expected that lithium may alter beta responses in cognitive networks. In addition to neuroplasticity cascades, lithium also reduces excitatory neurotransmission 
(dopamine or glutamate) and increases inhibitory neurotransmission (GABA) (Malhi et al., 2013). Given the fact that EEG signal consists of excitatory-end-synaptic potentials, then it would be quite possible that psychotropic medications may alter the EEG signal. Cognitive processes demand broader range of oscillations that may include fast frequencies as well as slow frequencies, thus beta oscillatory responses could be investigated under cognitive tasks.

\section{Methods}

\subsection{Subjects}

All patients were euthymic for at least six months. 22 patients were medication-free (19 Bipolar I, three Bipolar II disorder) and 16 patients were on lithium monotherapy. 21 healthy participants were enrolled to the control group (Table 1). Diagnoses were confirmed with the Structured Clinical Interview According to DSM-IV (SCID) (First et al., 1996). The Young Mania Rating Scale (YMRS) (Young et al., 1978) and Hamilton Depression Rating Scale (HDRS) (Hamilton, 1960) were the clinical evaluation tools. All subjects were right handed. Clinical data and previous history of the disorder was assessed by the psychiatrist, taking into account available charts and case notes. Patients enrolled into the lithium group were required to be on lithium monotherapy for at least eight weeks (Average $=114.87 \pm 208.06$ weeks, Range: $8-832$ weeks), and on effective serum lithium levels (Average $=0.79 \pm 0.12 \mathrm{mEq} / \mathrm{dl}$, Range: 0.59-1.08 mEq/dl). Exclusion criteria were: Comorbid axis I diagnosis, mental retardation, pregnancy, lactation, consumption of alcohol or substances two weeks prior to the recordings, axis-I psychiatric co-morbidity and neurological conditions such as neurodegenerative diseases, history of ECT in the last six months, epilepsy and brain surgery. Subjects were asked to avoid from sleep deprivation, not to smoke cigarettes or consume coffee or energy drinks two hours prior to the experiment. After providing a complete description of the study to the participants, written informed consent was obtained from each participant. The local Ethical Committee approved the study.

\subsection{Experiments}

Experiments were performed in a dimly-lit isolated and shielded room. Subjects were seated in front of a desk and two loud speakers were located at a $50 \mathrm{~cm}$ distance in front of the subject. Electrodes were located on an elastic cap (easy-cap) containing $30 \mathrm{Ag}-\mathrm{AgCl}$ electrodes according to the international 10-20 system. Two linked earlobe reference electrodes $\left(A_{1}-A_{2}\right)$ and electrooculography (EOG), placed on medial upper and lateral rim of the right eye, were used. All electrode impedances were lower than $10 \mathrm{k} \Omega$. The EEG was amplified by means of a 
BrainAmp 32-channel DC device with band limits of 0.01-250 Hz. The EEG was digitized online at a sampling rate of $500 \mathrm{~Hz}$.

Initially, simple stimuli were given in the first session, and in the second session the oddball paradigm, which included two types of auditory stimuli including target and non-target stimuli were presented to the subjects. The auditory simple stimuli were tones of $80 \mathrm{~dB}$ and 1,500 $\mathrm{Hz}$. The inter-stimulus intervals varied randomly between 3 and $7 \mathrm{~s}$. The total number of stimuli was 60 . In a random sequence, task-relevant target $(80 \mathrm{~dB}, 1600 \mathrm{~Hz}$ tone) and task-irrelevant non-target $(80 \mathrm{~dB}, 1500 \mathrm{~Hz})$ stimuli were the components of the classical auditory oddball paradigm (40 target, 80 non-target). The interval between tones varied randomly between three to seven seconds. Subjects were instructed to carefully listen, discriminate the two tones, and mentally count the number of target stimuli. Total counts were noted as behavioral data.

\subsection{Data Analysis}

EEG was segmented between -500 and $+1000 \mathrm{~ms}$ time period for each subject and for each stimulation. Then the artifacts due to eye blinks, detected by the EOG electrodes were eliminated by semi-automatic procedure in the Brain vision Analzyer program. The program showed us the excessive epochs which were above $200 \mathrm{~Hz}$ frequency and $50 \mu \mathrm{V}$ amplitude. We have manually removed the artifacts. Artifact free epochs then segmented again in the $0-800 \mathrm{~ms}$ time periods and averaged and FFT with a $10 \%$ Hanning window was applied to the averaged ERPs (Figure 1). Looking at the FFT we have determined the filter limits of the beta frequency window as 14-28 Hz. These information was use to filter the averaged ERP responses. The band pass filter was used with a 14-28 $\mathrm{Hz}$ frequency window and $48 \mathrm{~dB} / \mathrm{Oct}$ slope. The maxiumum peak to peak amplitude of filtered beta responses in $0-300 \mathrm{~ms}$ time period was then investigated for each subject, for each electrode and for each stimulation manually by the investigator. 
Figure 1. Power spectrum analyses with Fast Fourier Transform at beta frequency oscillations ( $\mathrm{F}_{4}$ location).
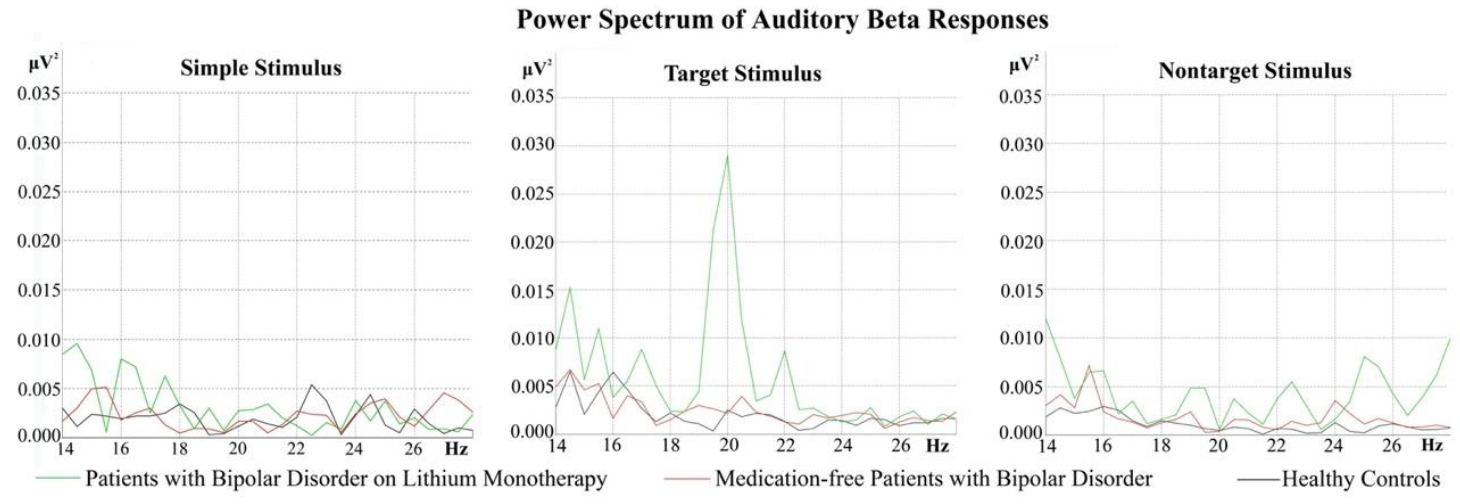

The amplitude of the largest peak on the filtered waveform within 0-300 ms between negative and positive maximum-peak points of the beta frequency oscillations was accepted as the maximum peak-to-peak amplitudes of the beta oscillations (Figure 2). Beta oscillations upon simple, target and non-target stimuli were measured in 0-300 ms time interval after stimuli (Figure 2). Electrodes locations were $\mathrm{F}_{3}, \mathrm{~F}_{4}, \mathrm{C}_{3}, \mathrm{C}_{4}, \mathrm{~T}_{7}, \mathrm{~T}_{8}, \mathrm{Tp}_{7}, \mathrm{Tp} \mathrm{p}_{8}, \mathrm{P}_{3}, \mathrm{P}_{4}, \mathrm{O}_{1}, \mathrm{O}_{2}$. Additionally, timing of the earlier one of the largest peaks was noted in order to compare latency of the beta oscillations between groups. 
Figure 2. Grand-averaged simple $\left(1^{\text {st }}\right.$ line $)$ and target $\left(2^{\text {nd }}\right.$ line $)$ beta responses upon auditory oddball stimulation ( $\mathrm{F}_{4}$ location). Healthy control group is shown with black lines, medicationfree patients with bipolar disorder are shown with red lines and patients with bipolar disorder on lithium monotherapy are shown with green lines. Responses to target stimuli in the lithium group are significantly higher than both healthy controls and medication-free patients.

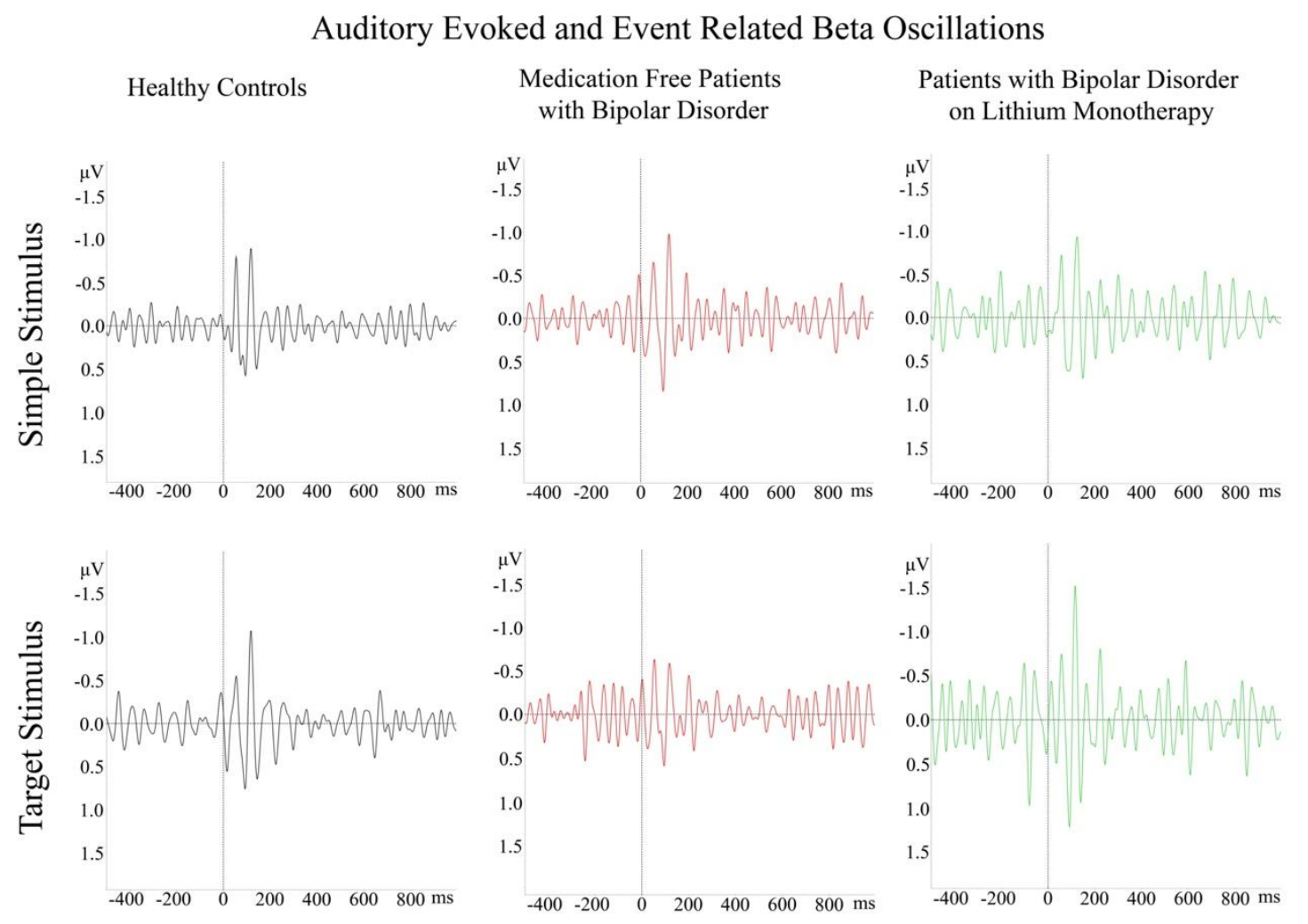

\subsection{Statistical Analysis}

Sociodemographic variables were compared with t-test or Mann Whitney U test for continuous variables according to the distribution characteristics. Categorical variables were analyzed with chi-square test. Repeated measures ANOVA were used to compare groups. Both ANOVAs included three groups (medication-free patients with bipolar disorder, healthy controls and patients with bipolar disorder on lithium monotherapy) as between subjects factor. Three stimuli (simple, target and non-target stimuli), two hemispheres (right and left) and six locations (frontal, central, temporal, temporoparietal, parietal and occipital) were used as within-subjects factor in the repeated measures ANOVA. All variances were homogeneous in the Levene's test, so Tukey and t tests were chosen for the post-hoc comparisons. Greenhouse-Geisser corrected $\mathrm{p}$ values were reported. Behavioral data were analyzed with one-way ANOVA. Correlations between the clinical and behavioral data and the EEG data were performed with Spearman's correlation test. 


\section{Results}

\subsection{Clinical Results}

As presented in Table 1, groups were similar in terms of age and gender. Education differed between groups $(\mathrm{p}<0.001)$. The healthy control group had significantly longer duration of education compared to the lithium and the drug-free patient groups. Duration of the disorder and number of episodes were higher in the lithium group, in comparison to the medication-free bipolar patient group. The HDRS scores differed significantly between patient groups; the medication-free group had higher scores than the lithium group $(\mathrm{p}=0.013)$. The lithium group had significantly higher number of total $(p=0.039)$, manic $(p=0.046)$ and depressive $(p=0.009)$ episodes than the medication-free group. The patients enrolled in the lithium group had optimal levels of serum lithium concentrations (mean $=0.803 \mathrm{mEq} / \mathrm{lt})$.

Table 1. Clinical and socio-demographic characteristics of the groups

\begin{tabular}{|c|c|c|c|c|c|c|}
\hline & & $\begin{array}{l}\text { Lithium group } \\
\qquad(\mathrm{n}=16)\end{array}$ & $\begin{array}{c}\text { Euthymic } \\
\text { Drug-free } \\
(\mathbf{n}=22)\end{array}$ & $\begin{array}{c}\text { Healthy } \\
\text { Controls } \\
(\mathrm{n}=21)\end{array}$ & $\mathbf{F} / \mathbf{z} / \chi^{2}$ & $\mathbf{p}$ \\
\hline \multicolumn{2}{|l|}{ Age } & $34.75 \pm 9.90$ & $30.82 \pm 6.46$ & $29.10 \pm 7.87$ & 2.33 & 0.107 \\
\hline \multicolumn{2}{|l|}{ Education ${ }^{a}$} & $9.88 \pm 4.44$ & $11.77 \pm 3.61$ & $14.67 \pm 2.22$ & 9.13 & $<0.001$ ** \\
\hline \multicolumn{2}{|l|}{ Gender $(\mathbf{f} / \mathbf{m})$} & $6 / 10$ & $7 / 15$ & $5 / 16$ & 0.83 & 0.660 \\
\hline \multicolumn{2}{|l|}{ HDRS } & $0.88 \pm 1.09$ & $2.45 \pm 2.22$ & & 2.62 & 0.013 \\
\hline \multicolumn{2}{|l|}{ YMRS } & $0.56 \pm 0.96$ & $0.73 \pm 1.28$ & & 0.43 & 0.668 \\
\hline \multicolumn{2}{|c|}{ Age at Disease Onset ${ }^{a}$} & $21.75 \pm 7.45$ & $21.86 \pm 6.30$ & & 0.51 & 0.960 \\
\hline \multicolumn{2}{|c|}{ Duration of the Disorder ${ }^{\mathrm{a}}$} & $13.36 \pm 6.02$ & $10.05 \pm 4.96$ & & -1.81 & 0.073 \\
\hline \multicolumn{2}{|c|}{ Duration of Euthymia ${ }^{b}$} & $37.75 \pm 45.69$ & $48.64 \pm 37.71$ & & 0.80 & 0.427 \\
\hline \multirow{5}{*}{$\begin{array}{l}\text { Number of } \\
\text { previous } \\
\text { episodes }\end{array}$} & Total & $7.31 \pm 6.49$ & $3.91 \pm 3.13$ & & -2.15 & 0.039 \\
\hline & Manic & $3.25 \pm 2.70$ & $1.82 \pm 1.56$ & & -2.07 & 0.046 \\
\hline & Depressive & $2.94 \pm 2.89$ & $1.09 \pm 1.11$ & & -2.75 & 0.009 \\
\hline & Hypomanic & $0.81 \pm 1.17$ & $0.77 \pm 1.19$ & & -0.10 & 0.919 \\
\hline & Mixed & $0.38 \pm 1.26$ & $0.23 \pm 0.53$ & & -0.49 & 0.623 \\
\hline
\end{tabular}

Serum Lithium Levels

$0.803 \pm 0.12$

(mEq/lt)

One-way ANOVA, t test, Mann-Whitney U test and $\chi^{2}$ tests were used. f/m: female/male *MannWhitney $U$ test ${ }^{* *}$ Posthoc Bonferroni test, Healthy controls $>$ drug-free patients, $p=0.022$, Healthy controls $>$ Lithium group, $p<0.001 .{ }^{a}$ Years, ${ }^{b}$ Months 


\subsection{Beta Frequency Results}

Target stimulus beta responses of the lithium group were $23.2 \%-36.2 \%$ higher than the medication-free bipolar patient group and $19.5 \%-40.6 \%$ higher than the healthy control group (Figure 3). The repeated measures ANOVA revealed significant group difference $[\mathrm{p}=0.001$, $\mathrm{F}(2,56)=8.14$; partial eta squared=0.225]. Patients on lithium therapy had higher responses than both medication-free patients $(\mathrm{p}=0.003)$ and healthy controls $(\mathrm{p}=0.002)$. There was a stimulus type effect $[\mathrm{p}<0.001, \mathrm{~F}(2.112)=17.39]$; post-hoc comparisons showed that target responses were significantly higher than both simple and non-target stimulus ( $\mathrm{p}<0.001$ for both). Repeated measures ANOVA also revealed a location type effect $[p<0.001, F(5,280)=13.43]$. Post-hoc comparison showed that frontal location had higher amplitudes than central, temporoparietal, parietal and occipital locations [ $<<0.001$ for all]. Central locations had higher amplitudes than temporoparietal, parietal, and occipital locations [ $\mathrm{p}<0.001$ for all]. Temporal locations had higher amplitudes than temporoparietal $[\mathrm{p}=0.001]$ locations. Laterality $\mathrm{X}$ group type effect was also significant $[\mathrm{p}=0.010, \mathrm{~F}(1,56)=4.99]$. Post-hoc comparisons for group $\mathrm{X}$ laterality type effect showed that the amplitudes of right hemispheric responses of the lithium group $(4.51 \mu \mathrm{V})$ were significantly higher than the right hemispheric responses of the healthy control group $([2.85 \mu \mathrm{V}] \mathrm{p}=0.001)$ and the medication-free patient group $([2.90 \mu \mathrm{V}] \mathrm{p}=0.001)$. In addition, there was no statistical difference between groups in terms of latency $[\mathrm{p}=0.749, \mathrm{~F}(1,56)=0.291]$. When duration of education is added to the analyses as a covariate, no difference between groups was observed in beta oscillations $[\mathrm{p}=0.084, \mathrm{~F}(1,55)=3.10]$. 
Figure 3. Event-related potentials (P300 responses to target stimulation). F4 location

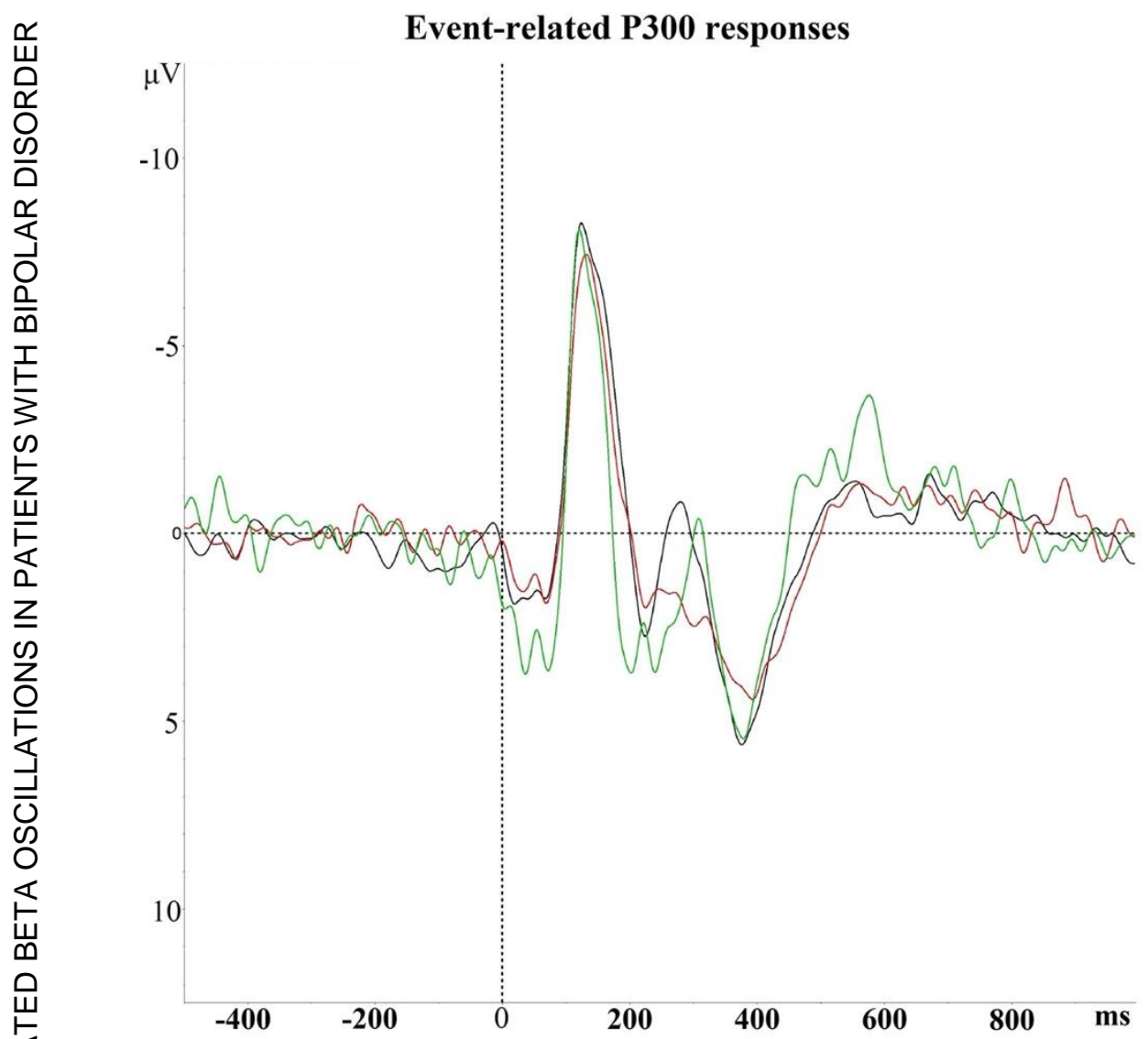


Figure 4. Beta frequency responses of all groups upon auditory simple and oddball paradigm. Maximum peak-to-peak amplitudes of subjects were measured and then averaged. The graphic represents Stimulus X Group X Location type effect. There was no statistically significant difference between groups in this type of interaction.

\section{Evoked and Event Related Beta Responses}

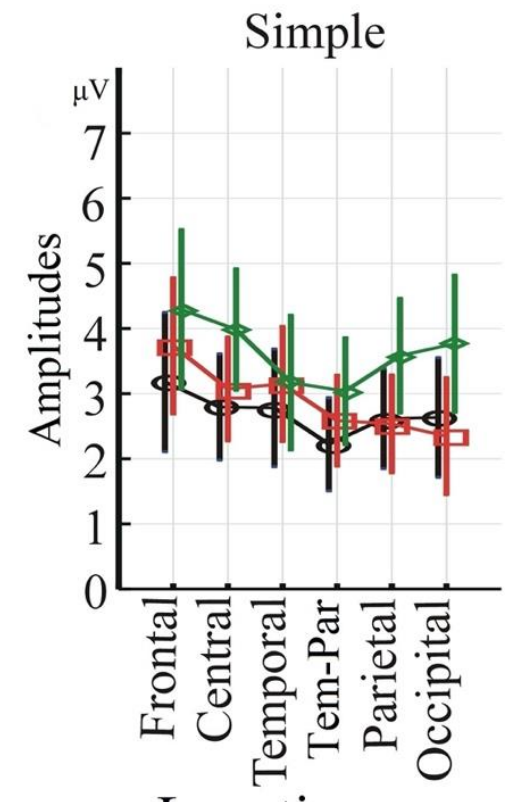

Locations

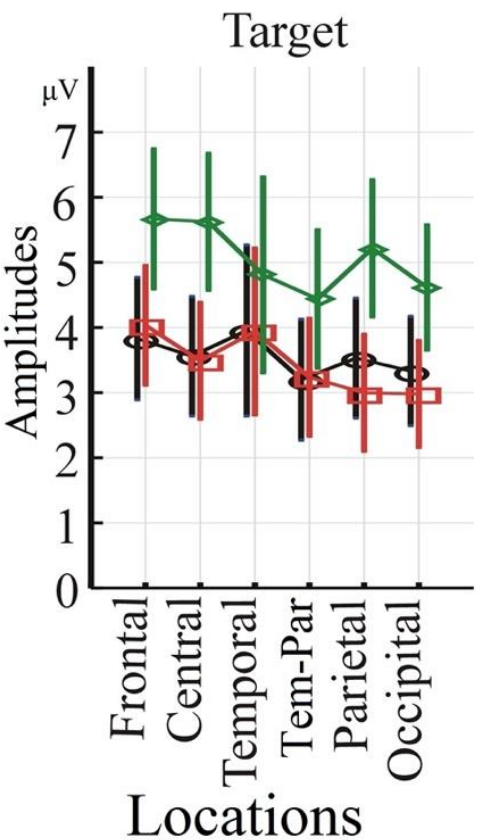

Locations

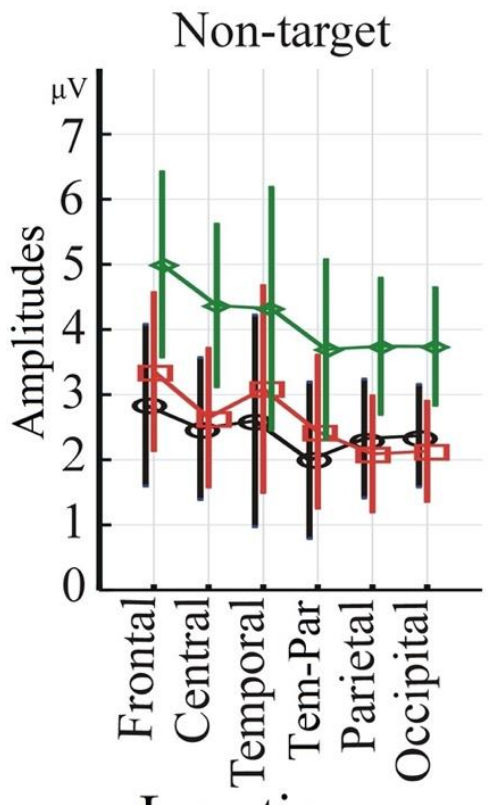

Locations

$\longrightarrow$ - Healthy Controls

$\neg \square$ Medication-Free Patients with Bipolar Disorder

$\curvearrowright$ Lithium Group

\subsection{Correlation analyses}

Correlation analyses showed correlations between clinical parameters and beta oscillatory responses (Table 2). 
Table 2. Correlations between clinical variables and amplitudes in the lithium group

\begin{tabular}{|c|c|c|c|}
\hline & Location & r value & p value \\
\hline Number of total episodes & $F_{3}$ target & 0.663 & 0.005 \\
\hline \multirow{6}{*}{ Number of depressive episodes } & F3 ep $_{3}$ & 0.581 & 0.018 \\
\hline & $\mathrm{F}_{4} \mathrm{ep}$ & 0.512 & 0.043 \\
\hline & $F_{3}$ target & 0.557 & 0.025 \\
\hline & F3 non-target & 0.587 & 0.017 \\
\hline & $O_{1}$ nontarget & 0.602 & 0.014 \\
\hline & $\mathrm{O}_{2}$ nontarget & 0.588 & 0.017 \\
\hline \multirow{12}{*}{ Serum lithium levels } & F3 $_{3}$ ep & -0.529 & 0.043 \\
\hline & $\mathbf{T}_{7} \mathbf{e p}$ & -0.532 & 0.041 \\
\hline & T8 ep & -0.586 & 0.022 \\
\hline & Tp8 ep & -0.821 & $<0.001$ \\
\hline & $\mathrm{O}_{1} \mathrm{ep}$ & -0.686 & 0.005 \\
\hline & $\mathrm{O}_{2} \mathrm{ep}$ & -0.614 & 0.015 \\
\hline & T8 target & -0.590 & 0.021 \\
\hline & Tps target & -0.661 & 0.007 \\
\hline & $\mathbf{T}_{7}$ non-target & -0.718 & 0.003 \\
\hline & T8 non-target & -0.614 & 0.015 \\
\hline & Tps non-target & -0.564 & 0.028 \\
\hline & $\mathrm{O}_{1}$ non-target & -0.545 & 0.036 \\
\hline
\end{tabular}

Spearman's correlation analyses. Variables in the correlation analyses: Amplitudes of beta oscillatory responses upon auditory simple and oddball stimuli, age, education, age at disorder onset, number of total/manic/depressive episodes, duration of the disorder, serum lithium levels, scores of Hamilton Depression Rating Scale and Young Mania Rating Scale.

Correlations between clinical variables and amplitudes of beta oscillations in the drug-free patients with bipolar disorder were duration of euthymia was correlated to non-target stimulus responses at $\mathrm{T}_{7}(\mathrm{r}=0.495, \mathrm{p}=0.019)$ and $\mathrm{Tp}_{7}(\mathrm{r}=0.560, \mathrm{p}=0.007)$ locations and HAM-D scores were correlated to simple stimulus responses at $\mathrm{F}_{4}(\mathrm{r}=-0.539, \mathrm{p}=0.010)$ and $\mathrm{O}_{1}(\mathrm{r}=-0.453$, $\mathrm{p}=0.034$ ) locations.

\subsection{Behavioral Data Analyses}

Behavioral data analyses showed that there were no difference between the lithium (37.94 \pm 7.49$)$, medication-free patients $(39.91 \pm 10.33)$ and healthy control (40.90 \pm 6.02$)$ groups in terms of the total number of mental counts of the target stimuli $(\mathrm{p}=0.564, \mathrm{~F}=0.580)$. 


\section{Discussion}

\subsection{Enhanced beta oscillations}

Event related beta responses of the lithium group were significantly higher than medicationfree patients and healthy controls. Previous resting EEG studies consistently showed amplified delta and theta activity; however, findings of this study suggest that cognition related networks are influenced by lithium as well as default mode networks. Findings of this study may reflect rearrangements in brain networks stimulated by lithium; however, it is not clear whether these EEG changes are correlates of neuroplastic processes. Many other factors may also be involved in the changes stimulated by an external manipulation as discussed below.

Differences in beta oscillations were significant only in target responses in this study and this finding may suggest that lithium influences cognitive activity in cognition based networks. Attention load of responses to target stimulations are higher than non-target stimulations, therefore the amount of the arisen energy during the processing of target stimulation should be higher than the amount of the energy of processing simple and non-target stimuli. Additionally, in target detection tasks, stimulus saliency has been associated with increased beta activity (Brenner et al., 2013). A recent study in patients with mild cognitive impairment reported that target responses were significantly greater than non-target responses in beta frequency band (Guntekin et al., 2013), supporting the idea that beta frequency responses are related to cognitive load and stimulus saliency. Beta oscillations are also related to attention (Marrufo et al., 2001), emotion processing (Güntekin and Başar, 2010) movements (Engel and Fries, 2010) and memory formation (Özgören et al., 2005; Başar et al., 2007; Weiss and Mueller, 2012).

Elevated baseline EEG activity might be a reason of increased activation energy in pharmacological neuroimaging studies. For example, increased signal/noise ratio in pharmacological neuroimaging studies could be related with hippocampal or cortical dysfunction as well as GABA and glutamatergic dysfunction in schizophrenia (Gandal et al., 2012). GABAergic system is also disturbed in bipolar disorder (Petty, 1995; Benes and Beretta, 2001; Brady et al., 2013) and therefore modulation of bioelectrical activity may be disturbed in bipolar disorder as well. This approach also suggests that increased amplitudes by lithium might be related to the noise due to the elevated background brain activity as well as other effects of lithium on networks. Lithium inhibits presynaptic $\alpha_{2}$ receptors non-selectively (Lenox and Hahn, 2000; Manji et al., 1991) so inhibition of task irrelevant brain cites may become harder and thus signal/noise ratio might be altered. Modulation of the electrical activity or -fine tuningis a function of GABAergic interneurons (Basar and Guntekin, 2008; Buzsaki, 2006) and it is 
possible that dysfunction of GABAergic neurons may negatively influence the modulation of the activity and excessively increase the responses.

Although lithium does not deteriorate cognitive functions (Wingo et al., 2009; BalanzáMartínez et al., 2010), except some mild disturbances in motor functions (Wingo et al., 2009; Atagün et al., 2013a); the question to be answered here is: are these excessively increased amplitudes related to an effect, or a cognitive side effect of lithium? Clinical data of this crosssectional study is not sufficient to extract consequences about clinical response to lithium. Although it has been largely accepted that lithium has neuroprotective and neurotrophic effects (Kim and Thayer, 2009), from the perspective of the neurotoxicity hypothesis (Fountoulakis et al., 2008) excessively enhanced beta oscillations could be evaluated as an adverse effect.

\subsection{Correlation Data}

Serum lithium levels were negatively correlated with beta frequency responses. This negative correlation suggests a dose-dependent amplification of activation energy in the networks. Low doses of lithium may not stimulate GABAergic neurotransmission as much as high concentrations (Otero-Lasada and Rubio, 1986). GABAergic dysfunction in bipolar disorder (Petty, 1995) is suggested to be the reason of the noise increase in neuroimaging studies in bipolar disorder (Gandal et al., 2012) and lithium may reverse the GABAergic dysfunction in a dose dependent manner. If so, then it will be possible to speculate that higher doses of lithium might be more beneficial than lower doses. Relations of serum lithium concentrations and brain oscillations deserve to be pursued in future studies to investigate underlying mechanisms. These mechanisms potentially contribute to optimize treatment strategies of lithium.

\subsection{Limitations}

The lithium and the medication-free bipolar patient groups were not matched in terms of education and clinical data. Target responses are related to cognitive processes and thus education difference may become an important limitation, because main differences in this study were at event-related beta oscillations. Patients of the lithium group had higher number of previous episodes, which may suggest more severe disorder than patients of the medicationfree group. Besides, evaluation of clinical lithium response with follow up may be useful. Many confounders like episodic nature of the disorder, medication effects, subthreshold symptoms, and difficulty in obtaining homogeneous samples, may preclude obtaining consistent results when studying bipolar disorder. Therefore, studying with euthymic, medication-free patients with bipolar disorder is the major strength of this study. 


\subsection{Conclusion Remarks}

This is the first study to evaluate effects of lithium on oscillatory brain responses to auditory simple and oddball paradigm, and the study showed that lithium increases the amplitudes of the auditory event-related beta-responses in the target stimulus condition. These findings are in line with the previous studies that reported increased white matter connectivity (Benedetti et al, 2013), increased gray matter volumes and density (Bearden et al., 2007, Moore et al. 2000a) or improvements in brain chemistry (Moore et al., 2000b) by lithium. The correlation data may suggest that higher doses of lithium might be more useful than low doses. Just as the earlier attempt (Hegerl et al., 1987), future studies should focus on the clinical response to lithium and cognitive functionality in lithium EEG studies. This method may potentially facilitate predicting or evaluating dose and effectiveness of lithium in bipolar disorder. For the further steps in search of improved treatments, treatments should be considered in a broader perspective including neurophysiological aspects (Kam et al., 2011), to achieve greater success in the treatment of psychiatric disorders.

\section{References}

Arai, J., Natsume, K., 2006. The properties of carbachol-induced beta oscillation in rat hippocampal slices. Neurosci Res. 54, 95103.

Atagun, M.I., Balaban, O.D., Lordoglu, D.Y., Evren, E.C., 2013a. Lithium and valproate may affect motor and sensory speed in patients with bipolar disorder. Bulletin of Clinical Psychopharmacology 23, 305-314.

Atagün, M.İ., Güntekin, B., Ozerdem, A., Tülay, E., Başar, E., 2013b. Decrease of theta response in euthymic bipolar patients during an oddball paradigm. Cogn Neurodyn. 7, 213-223.

Atagün, M.İ., Güntekin, B., Maşalı, B., Tülay, E., Başar, E., 2014. Decrease of EventRelated Delta Oscillations in Euthymic Patients with Bipolar Disorder. Psychiatry Res Neuroimaging. 223, 43-48.
Balanzá-Martínez, V., Selva, G., MartínezArán, A., Prickaerts, J., Salazar, J., González-Pinto, A., Vieta, E., TabarésSeisdedos, R., 2010. Neurocognition in bipolar disorders--a closer look at comorbidities and medications. Eur $\mathrm{J}$ Pharmacol. 626, 87-96.

Başar, E., 1998. Brain Functions and Oscillations Volume I: Brain Oscillations Principals and Approaches. Springer, Berlin, pp. 293-324.

Başar, E., 1999. Brain Functions and Oscillations II: Integrative Brain Function Neurophysiology and Cognitive Processes. Springer, Berlin, pp. 393-402.

Başar, E., Başar-Eroglu, C., Karakaş, S., Schürmann, M., 2001. Gamma, alpha, delta, and theta oscillations govern cognitive processes. Int J Psychophysiol. 39, 241-248. 
Başar, E., 2006. The theory of the whole-brainwork. Int J Psychophysiol. 60, 133-138.

Başar, E., Ozgören, M., Oniz, A., Schmiedt, C., Başar-Eroğlu, C., 2007. Brain oscillations differentiate the picture of one's own grandmother. Int J Psychophysiol. 64, 8190.

Başar, E., Güntekin, B., 2008. A review of brain oscillations in cognitive disorders and the role of neurotransmitters. Brain Res.1235, 172-193.

Başar, E., 2010. Brain-Body-Mind in the Nebulous Cartesian System: A holistic approach by oscillations. Springer, New York.

Başar, E., Güntekin, B., Atagün, I., TurpGölbaşı, B., Tülay, E., Ozerdem, A., 2012. Brain's alpha activity is highly reduced in euthymic bipolar disorder patients. Cogn Neurodyn. 6, 11-20.

Başar, E., Başar-Eroğlu, C., Güntekin, B., Yener, G.G., 2013. Brain's alpha, beta, gamma, delta, and theta oscillations in neuropsychiatric diseases: proposal for biomarker strategies. Suppl Clin Neurophysiol. 62, 19-54.

Başar, E., Güntekin, B., 2013. Review of delta, theta, alpha, beta, and gamma response oscillations in neuropsychiatric disorders. Suppl Clin Neurophysiol. 62, 303-341.

Bearden, C.E., Thompson, P.M., Dalwani, M., Hayashi, K.M., Lee, A.D., Nicoletti, M., Trakhtenbroit, M., Glahn, D.C., Brambilla, P., Sassi, R.B., Mallinger, A.G., Frank, E., Kupfer, D.J., Soares, J.C., 2007. Greater cortical gray matter density in lithium treated patients with bipolar disorder. Biol Psychiatry. 62, 7-16.

Benes, F.M., Berretta, S., 2001. GABAergic interneurons: Implications for understanding schizophrenia and bipolar disorder. Neuropsychopharmacology 25, 1-27.

Benedetti, F., Bollettini, I., Barberi, I., Radaelli, D., Poletti, S., Locatelli, C., Pirovano, A., Lorenzi, C., Falini, A., Colombo, C., Smeraldi, E., 2013 Lithium and GSK3- $\beta$ promoter gene variants influence white matter microstructure in bipolar disorder. Neuropsychopharmacology 38, 313-27.

Brady, R.O. Jr1., McCarthy, J.M., Prescot, A.P., Jensen, J.E., Cooper, A.J., Cohen, B.M., Renshaw, P.F., Ongür, D., 2013. Brain gamma-aminobutyric acid (GABA) abnormalities in bipolar disorder. Bipolar Disord. 15, 434-439.

Brenner, C.A., Kieffaber, P.D., Clementz, B.A., Johannesen, J.K., Shekhar, A., O'Donnell, B.F., Hetrick, W.P., 2009. Event-related potential abnormalities in schizophrenia: a failure to "gate in" salient information? Schizophr Res. 113, 332-338.

Bruns, A., Eckhorn, R., 2004. Task-related coupling from high- to low-frequency signals among visual cortical areas in human subdural recordings. Int J Psychophysiol. 51, 97-116.

Buzsaki, G., 2006. Rhythms of the brain. Oxford University Press, USA, pp. 61-79.

Engel, A.K., Fries, P., 2010. Beta-band oscillations--signalling the status quo? Curr Opin Neurobiol. 20, 156-165.

Ethridge, L.E., Hamm, J.P., Shapiro, J.R., Summerfelt, A.T., Keedy, S.K., Stevens, M.C., Pearlson, G., Tamminga, C.A., Boutros, N.N., Sweeney, J.A., Keshavan, M.S., Thaker, G., Clementz, B.A., 2012. Neural activations during auditory oddball processing discriminating schizophrenia and psychotic bipolar disorder. Biol Psychiatry. 72, 766-774. 
First, M.B., Gibbon, M., Spitzer, R.L., Gibbon, M., Williams, J.B.W., 1996. User's guide for the structured interview for DSM-IV axis I disorders-research version (SCID-I, version 2.0, February 1996 final version). Biometrics Research, New York.

Fountoulakis, K.N., Vieta, E., Bouras, C., Notaridis, G., Giannakopoulos, P., Kaprinis, G., Akiskal, H., 2008. A systematic review of existing data on long-term lithium therapy: neuroprotective or neurotoxic? Int J Neuropsychopharmacol. 11, 269-287.

Gandal, M.J., Edgar, J.C., Klook, K., Siegel, S.J., 2012. Gamma synchrony: Towards a translational biomarker for the treatmentresistant symptoms of schizophrenia. Neuropharmacology 62, 1504-1518.

Güntekin, B., Başar, E., 2010. Event-related beta oscillations are affected by emotional eliciting stimuli. Neurosci Lett. 483, 173178.

Güntekin, B., Emek-Savaş, D.D., Kurt, P., Yener, G.G., Başar, E., 2013. Beta oscillatory responses in healthy subjects and subjects with mild cognitive impairment. Neuroimage Clinical. 3, 39-46.

Hamilton, M., 1960. A rating scale for depression. J. Neurol. Neurosur. Ps. 23, 5662.

Hamm, J.P., Ethridge, L.E., Shapiro, J.R., Stevens, M.C., Boutros, N.N., Summerfelt, A.T., Keshavan, M.S., Sweeney, J.A., Pearlson, G., Tamminga, C.A., Thaker, G., Clementz, B.A., 2012. Spatiotemporal and frequency domain analysis of auditory paired stimuli processing in schizophrenia and bipolar disorder with psychosis. Psychophysiology 49, 522-530.

Hegerl, U., Ulrich, G., Müller-Oerlinghausen, B., 1987. Auditory evoked potentials and response to lithium prophylaxis. Pharmacopsychiatry 20, 213-216.
Hyun, J., Baik, M.J., Kang, U.G., 2011. Effects of Psychotropic Drugs on Quantitative EEG among Patients with Schizophreniaspectrum Disorders. Clin Psychopharmacol Neurosci. 9, 78-85.

Kam, J.W.Y., Bolbecker, A.R., O’Donnell, B.F., Hetrick, W.P., Brenner, C.A., 2011. Prospective predictors of mood episodes in bipolar disorder. J Affect Disord. 135, 298304.

Karniol, I.G., Dalton, J., Lader, M.H., 1978. Acute and chronic effects of lithium chloride on physiological and psychological measures in normals. Psychopharmacology (Berl). 57, 289-294.

Kenemans, J.L., Kähkönen, S., 2011. How human electrophysiology informs psychopharmacology: from bottom-up driven processing to top-down control. Neuropsychopharmacology 36, 26-51.

Kim, H.J., Thayer, S.A., 2009. Lithium increases synapse formation between hippocampal neurons by depleting phosphoinositides. Mol Pharmacol. 75, 1021-1030.

Kirschner, A., Kam, J.W., Handy, T.C., Ward, L.M., 2012 Differential synchronization in default and task-specific networks of the human brain. Front Hum Neurosci. 6, 139.

Lee, P.S., Chen, Y.S., Hsieh, J.C., Su, T.P., Chen, L.F., 2010. Distinct neuronal oscillatory responses between patients with bipolar and unipolar disorders: a magnetoencephalographic study. J Affect Disord. 123, 270-275.

Lenox, R.H., Hahn, C.G., 2000. Overview of the mechanism of action of lithium in the brain: fifty-year update. J Clin Psychiatry. 61, 5-15.

Malhi, G.S., Tanious, M., Das, P., Coulston, C.M., Berk, M., 2013. Potential mechanisms 
of action of lithium in bipolar disorder. Current understanding. CNS Drugs. 27, 135153.

Manji, H.K., Hsiao, J.K., Risby, E.D., Oliver, J., Rudorfer, M.V., Potter, W.Z., 1991. The mechanisms of action of lithium. I. Effects on serotoninergic and noradrenergic systems in normal subjects. Arch Gen Psychiatry. 48, 505-512.

Moore, G.J., Bebchuk, J.M., Wilds, I.B., Chen, G., Manji, H.K., 2000a. Lithium-induced increase in human brain grey matter. Lancet $356,1241-1242$.

Moore, G.J., Bebchuk, J.M., Hasanat, K., Chen, G., Seraji-Bozorgzad, N., Wilds, I.B., Faulk, M.W., Koch, S., Glitz, D.A., Jolkovsky, L., Manji, H.K., 2000b. Lithium increases Nacetyl-aspartate in the human brain: in vivo evidence in support of bcl-2's neurotrophic effects? Biol Psychiatry. 48, 1-8.

Moussa, M.N., Vechlekar, C.D., Burdette, J.H., Steen, M.R., Hugenschmidt, C.E., Laurienti, P.J., 2011. Changes in cognitive state alter human functional brain networks. Front Hum Neurosci. 5, 83.

O'Donnell, B.F., Hetrick, W.P., Vohs, J.L., Krishnan, G.P., Carroll, C.A., Shekhar, A., 2004. Neural synchronization deficits to auditory stimulation in bipolar disorder. Neuroreport 15, 1369-1372.

Otero Losada, M.E., Rubio, M.C., 1986. Acute and chronic effects of lithium chloride on GABA-ergic function in the rat corpus striatum and frontal cerebral cortex. Naunyn. Schmiedebergs Arch Pharmacol. 332, 169-172.

Özerdem, A., Güntekin, B., Tunca, Z., Başar, E., 2008. Brain oscillatory responses in patients with bipolar disorder manic episode before and after valproate treatment. Brain Res. 1235, 98-108.
Özerdem, A., Güntekin, B., Saatçi, E., Tunca, Z., Başar, E., 2010. Disturbance in long distance gamma coherence in bipolar disorder. Prog. Neuropsychopharmacol. Biol Psychiatry. 34, 861-865.

Özerdem, A., Güntekin, B., Atagün, I., Turp, B., Başar, E., 2011. Reduced long distance gamma $(28-48 \mathrm{~Hz})$ coherence in euthymic patients with bipolar disorder. J Affect Disord. 132, 325-332.

Özgören, M., Başar-Eroğlu, C., Başar, E., 2005. Beta oscillations in face recognition. Int $\mathbf{J}$ Psychophysiol. 55, 51-59.

Petty, F., 1995. GABA and mood disorders: a brief review and hypothesis. J Affect Disord. 34, 275-281.

Rass, O., Krishnan, G., Brenner, C.A., Hetrick, W.P., Merrill, C.C., Shekhar, A., O'Donnell, B.F., 2010. Auditory steady state response in bipolar disorder: relation to clinical state, cognitive performance, medication status, and substance disorders. Bipolar Disord. 12, 793-803.

Schulz, C., Mavrogiorgou, P., Schröter, A., Hegerl, U., Juckel, G., 2000. Lithiuminduced EEG changes in patients with affective disorders. Neuropsychobiology 42 , 33-37.

Soeiro-de-Souza, M.G., Dias, V.V., Figueira, M.L., Forlenza, O.V., Gattaz, W.F., Zarate, C.A. Jr., Machado-Vieira, R., 2012. Translating neurotrophic and cellular plasticity: from pathophysiology to improved therapeutics for bipolar disorder. Acta Psychiatr Scand. 126, 332-341.

Thau, K., Rappelsberger, P., Lovrek, A., Petsche, H., Simhandl, C., Topitz, A., 1989. Effect of lithium on the EEG of healthy males and females. A probability mapping study. Neuropsychobiology 20, 158-163. 
Traub, R.D., Bibbig, A., LeBeau, F.E., Buhl, E.H., Whittington, M.A., 2004. Cellular mechanisms of neuronal population oscillations in the hippocampus in vitro. Annu Rev Neurosci. 27, 247-278.

Ulrich, G., Frick, K., Stieglitz, R.D., MüllerOerlinghausen, B., 1987. Interindividual variability of lithium-induced EEG changes in healthy volunteers. Psychiatry Res. 20, 117-127.

Ulrich, G., Herrmann, W.M., Hegerl, U., Müller-Oerlinghausen, B., 1990. Effect of lithium on the dynamics of electroencephalographic vigilance in healthy subjects. J Affect Disord. 20, 19-25.

Vázquez Marrufo, M., Vaquero, E., Cardoso, M.J., Gómez, C.M., 2001. Temporal evolution of alpha and beta bands during visual spatial attention. Brain Res Cogn Brain Res. 12, 315-320.
Weiss, S., Mueller, H.M., 2012. "Too many betas do not spoil the broth": The role of beta brain oscillations in language processing. Front Psychol. 3, 201.

Whittington, M.A., Faulkner, H.J., Doheny, H.C., Traub, R.D., 2000. Neuronal fast oscillations as a target site for psychoactive drugs. Pharmacol Ther. 86, 171-190.

Wingo, A.P., Wingo, T.S., Harvey, P.D., Baldessarini, R.J., 2009. Effects of lithium on cognitive performance: a meta-analysis. $\mathrm{J}$ Clin Psychiatry. 70, 1588-1597.

Young, R.C., Biggs, J.T., Ziegler, V.E., Meyer, D.A., 1978. A rating scale for mania: reliability, validity and sensitivity. $\mathrm{Br} \mathrm{J}$ Psychiatry 133, 429-435.

Zakowska-Dabrowska, T., Rybakowski, J., 1973. Lithium-induced EEG changes: relation to lithium levels in serum and red blood cells. Acta Psychiatr Scand. 49, 457465 


\section{CHAPTER 5}

\section{Meta-analysis of Auditory P50 Sensory Gating in Schizophrenia and Bipolar Disorder}

Murat İlhan Atagün, Marjan Drukker, Mei Hua Hall, Illkay Keleş Altun, Safiye Zeynep Tatlı, Sinan Gülöksüz, Jim van Os, Thérèse van Amelsvoort

Psychiatry Research: Neuroimaging. 2020; 300: 111078 


\section{Abstract}

The ability of the brain to reduce the amount of trivial or redundant sensory inputs is called gating function. Dysfunction of sensory gating may lead to cognitive fragmentation and poor real-world functioning. The auditory dual-click paradigm is a pertinent neurophysiological measure of sensory gating function. This meta-analysis aimed to examine the subcomponents of abnormal P50 waveforms in bipolar disorder and schizophrenia to assess P50 sensory gating deficits and examine effects of diagnoses, illness states (first-episode psychosis vs schizophrenia, remission vs episodes in bipolar disorder), and treatment status (medication-free vs medicated). Literature search of PubMed between Jan $1^{\text {st }} 1980$ and March 31st 2019 identified 2091 records for schizophrenia, 362 for bipolar disorder. 115 studies in schizophrenia (4932 patients), 16 in bipolar disorder (975 patients) and 10 in first-degree relatives (848 subjects) met the inclusion criteria. P50 sensory gating ratio (S2/S1) and S1-S2 difference were significantly altered in schizophrenia, bipolar disorder and their first-degree relatives. Firstepisode psychosis did not differ from schizophrenia, however episodes altered P50 sensory gating in bipolar disorder. Medications improve P50 sensory gating alterations in schizophrenia significantly and at trend level in bipolar disorder. Future studies should examine longitudinal course of P50 sensory gating in schizophrenia and bipolar disorder.

Key Words: EEG, sensory gating, bipolar disorder, schizophrenia 


\section{Introduction}

The ability to reduce the amount of trivial or redundant sensory inputs is called sensory gating function (Baker et al., 1987; Boutros et al., 1991; Freedman et al., 1997). Diminished sensory gating may lead to cognitive fragmentation and poor real-world functioning (Ancin et al., 2011; Johannesen et al., 2013; Martin et al., 2007b). The auditory dual-click (also called conditioning-testing task) paradigm is a pertinent neurophysiological measure of the sensory gating function, which represents a good marker for translational and clinical studies (Smucny et al., 2013). In the dual-click paradigm, paired successive clicks (separated by an interval of 500 milliseconds) are presented and two positive-polarity brain responses occurring approximately between 45-75 milliseconds after the stimuli (P50 potential) are measured. An example of typical normal responses to dual-click paradigm is shown in Figure 1. The relative decrease of the P50 waveform to the second click (S2) compared with the first (S1) is typically used as a measure of P50 sensory gating and quantified as the S2/S1 ratio or S1-S2 difference.

Figure 1. An example of typical normal responses to dual-click paradigm. The left column shows response to the conditioning (S1) click and the right column shows response to the testing (S2) click. The P50 ERP wave is marked by two vertical lines. In a dual-click paradigm experiment, a set of stimuli consisting of click-pairs (two successive clicks, S1 and S2 stimulus with an interval of $500 \mathrm{~ms}$ between the click-pairs) is presented to the subject and the responses of the second stimulus (S2) compared to the first stimulus (S1) of the pair is proposed to be the neurophysiological manifestation of the gating function. The Figure is adopted from Hall et al. 2008.
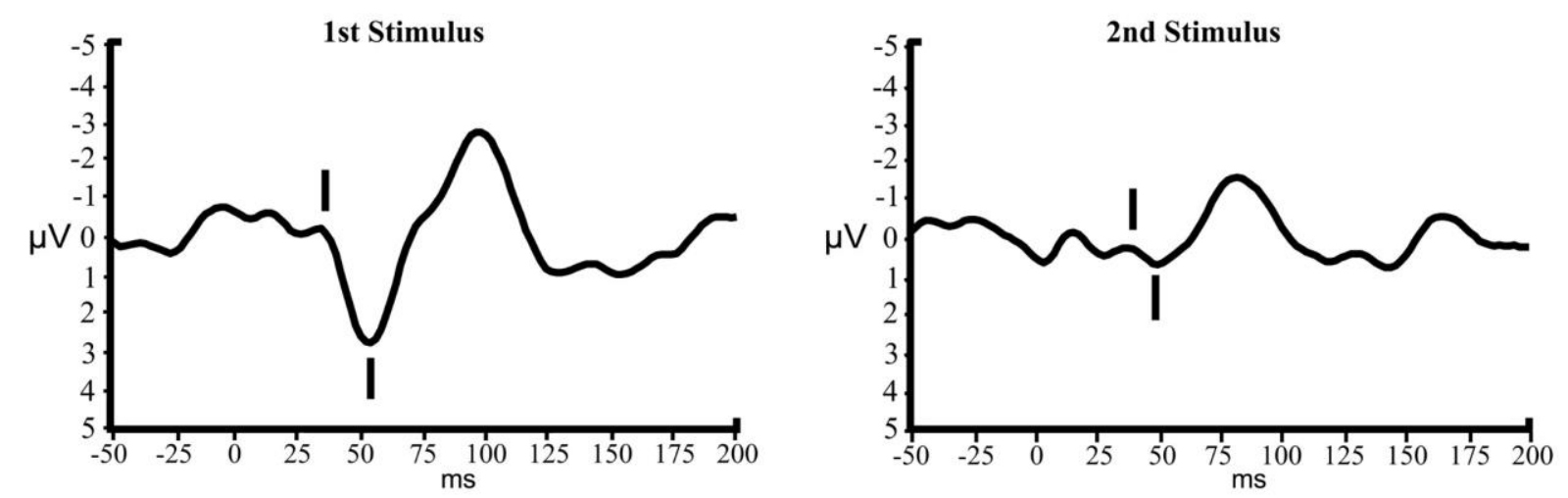
It is believed that hippocampus and prefrontal cortices participate in the inhibitory processes underlying mechanisms of P50 sensory gating (Bak et al., 2014). Neurobiological mechanisms of sensory gating deficit and its clinical associations have been widely investigated in schizophrenia and bipolar disorder (Hall et al., 2008; Jin et al., 1998; Johannesen et al., 2013; Martin et al., 2007a); however, findings have been inconsistent that might be partly due to confounding effects of medications, clinical symptoms (positive or negative symptoms), neuropsychological test performance and illness state (Potter et al., 2006; Swann et al., 2013; Vuillier et al., 2015). Despite clinical variations, schizophrenia and bipolar disorder may share similar pathological mechanisms in their etiology (Guloksuz and van Os, 2018; Kato, 2019; Lieberman and First, 2018) leading to similarities in research findings (Tamminga et al., 2013). Consistently, electroencephalography (EEG) studies with schizophrenia, bipolar disorder and their first-degree relatives revealed overlap in auditory-oddball paradigm (Mokhtari et al., 2016; Narayanan et al., 2015) or resting-state (Narayanan et al., 2014) EEG alterations. Likewise, it has been shown that sensory gating examinations with dual-click paradigm are altered in both schizophrenia and bipolar disorder (Bramon et al., 2004; Cheng et al., 2016; de Wilde et al., 2007b; Patterson et al., 2008). However, despite similarities between schizophrenia and bipolar disorder suggesting the disorders to be on a continuum and likely overlapping in etiology, variation in clinical symptoms and medications are suggestive of partially distinct pathological mechanisms sensory gating. Various underlying mechanisms might be involved with different components of P50 sensory gating ratio, for example amplitudes of S1 and S2 waveforms might be differentially influenced by neural synchrony and inhibitory neural circuits (Adler et al., 1994; Aidelbaum et al., 2018; Ancin et al., 2011; Freedman, 2014; Freedman et al., 1987; Oranje and Glenthoj, 2014). Medications and clinical progress may influence the P50 in patients with schizophrenia and bipolar disorder. Meta-analyses have confirmed a robust P50 sensory gating alteration in schizophrenia (Bramon et al., 2004; Chang et al., 2011; Cheng et al., 2016; de Wilde et al., 2007b; Patterson et al., 2009). However, 38 new studies (with 2230 schizophrenia patients, 1809 healthy controls) are published since the last meta-analysis in schizophrenia (Chang et al., 2011). There is only one meta-analysis of P50 sensory gating in bipolar disorder, which also reports robust P50 S2/S1 ratio alterations (Cheng et al., 2016).

Each meta-analysis report published thus far focused on only single disorder (schizophrenia or bipolar disorder). To our knowledge, no meta-analysis includes studies of both disorders to compare and evaluate the sensory gating alterations in patients with either disorder. In addition, only one meta-analysis report has included the unaffected relatives of patients to examine 
whether P50 sensory gating deficit is an endophenotype for schizophrenia and bipolar disorder $\underset{山}{\stackrel{\Upsilon}{山}} \quad$ (Bramon et al., 2004). This study aimed to extend previous meta-analyses to 1) clarify the magnitude of sensory gating alterations in bipolar disorder and schizophrenia compared to healthy controls; 2) examine the differences in P50 sensory gating alterations between illness states; 3) examine the differences in P50 sensory gating alterations between patients who were medicated and those who were drug-free; 4) evaluate the differences in P50 sensory gating between bipolar disorder patients in remission and those in episodes. Finally, to evaluate whether P50 sensory gating deficit is an endophenotype for schizophrenia and bipolar disorder, we presented a meta-analysis of first-degree relatives in this report.

\section{Methods}

The meta-analysis was performed according to recommendations of the Meta-analysis of Observational Studies in Epidemiology (MOOSE) group (Stroup et al., 2000). A review protocol, structured according to the PRISMA statement (Moher et al., 2009) was followed for data acquisition, analysis and reporting procedures.

\subsection{Search and study selection}

We performed a systematic literature search in Medline database. Human species, English language and publication dates (between Jan 1st 1980 and March 31st, 2019) were the activated filters. The key words [(schizophrenia OR psychosis) AND (P50 OR sensory gating)] retrieved 2091 records in schizophrenia and keywords [(bipolar disorder OR mania) AND (P50 OR sensory gating)] retrieved 362 records in bipolar disorder (Please see the flow-chart in Figure 2 for details). Studies were assessed according to the following criteria: I. Study groups should include a healthy control group and a schizophrenia and/or a bipolar disorder group, II. Electroencephalography should be recorded in the study, III. Paradigm of the study should be the dual-click paradigm, IV. Data analysis should be performed with a classical P50 analysis within the 1-20 Hz frequency range, V. Means and standard deviations should be reported in the article. Of the schizophrenia studies, 1921 were excluded after reading the titles and abstracts and 29 were eliminated after reading the full-text because of methodological reasons [i.e. difference in paradigm, data analysis, patient sample and device (magnetoencephalography studies were excluded)]. In addition, 26 articles were excluded in the next step because of inappropriate data (i.e. studies that do not report means and standard deviations were excluded). Of the bipolar disorder studies, 332 were excluded after reading the titles and abstracts. 8 studies were excluded because of methodological differences and 6 studies were excluded because of 
inappropriate data. In addition, studies with overlapping samples were excluded from further analysis, furthermore only first estimation was included from the studies that had repeated estimations of P50 sensory gating in a group. 115 articles (Adler et al., 2005; Adler et al., 1990a; Adler et al., 2004; Adler et al., 1982; Adler et al., 1985; Adler et al., 1990b; Arango et al., 2003; Arnfred et al., 2003; Arnfred et al., 2004; Bak et al., 2014; Baker et al., 1987; Becker et al., 2004; Becker et al., 2011; Boutros et al., 1999; Boutros et al., 2009; Boutros et al., 2004; Boutros et al., 1991; Braff et al., 2007; Brenner et al., 2009; Brockhaus-Dumke et al., 2008a; Brockhaus-Dumke et al., 2008b; Brunstein et al., 2005; Buchanan et al., 2003; Cabranes et al., 2013; Chen et al., 2011; Clementz and Blumenfeld, 2001; Clementz et al., 1997a; Clementz et al., 1997b, 1998a, b; Cullum et al., 1993; Dalecki et al., 2016; de Wilde et al., 2007a; Demily et al., 2016; Devrim-Ucok et al., 2008; Domjan et al., 2012; During et al., 2014; El-Kaim et al., 2015; Franks et al., 1983; Freedman et al., 1987; Fresan et al., 2007; Ghisolfi et al., 2006; Ghisolfi et al., 2004; Ghisolfi et al., 2002; Gjini et al., 2010; Gjini et al., 2011; Griffith and Freedman, 1995; Griffith et al., 1998; Griffith et al., 1993; Hall et al., 2015a; Hall et al., 2014; Hong et al., 2008a; Hong et al., 2004; Hong et al., 2007; Hong et al., 2009; Hsieh et al., 2004; Jin et al., 1998; Johannesen et al., 2005; Johannesen et al., 2013; Judd et al., 1992; Kathmann and Engel, 1990; Keri et al., 2010; Kim et al., 2018; Kisley and Cornwell, 2006; Koike et al., 2005; Light et al., 2000; Louchart-de la Chapelle et al., 2005a; Louchart-de la Chapelle et al., 2005b; Lu et al., 2007; Martin et al., 2007a; Mazhari et al., 2011; Micoulaud-Franchi et al., 2015a; Micoulaud-Franchi et al., 2012; Micoulaud-Franchi et al., 2015b; Micoulaud-Franchi et al., 2014; Micoulaud-Franchi et al., 2015c; Moran et al., 2012; Myles-Worsley, 2002; Nagamoto et al., 1996; Nagamoto et al., 1999; Nagamoto et al., 1989; Nagamoto et al., 1991; Neuhaus et al., 2014; Olincy et al., 2010; Olincy and Martin, 2005; Olincy et al., 2000; Oranje and Glenthoj, 2014; Patterson et al., 2009; Price et al., 2006; Rentzsch et al., 2007; Ringel et al., 2004; Sanchez-Morla et al., 2008; Sanchez-Morla et al., 2013; Sanchez-Morla et al., 2009; Santos et al., 2010; Shan et al., 2013; Siegel et al., 1984; Smith et al., 2010; Smith et al., 2013; Smucny et al., 2013; Song et al., 2014; Storozheva et al., 2016; Thoma et al., 2003; Thoma et al., 2008; Toyomaki et al., 2015; Turetsky et al., 2009; Vinogradov et al., 1996; Waldo et al., 1992; Waldo et al., 1988; Waldo et al., 1994; Waldo et al., 2010; Waters et al., 2009; Williams et al., 2011; Wonodi et al., 2014; Yee et al., 1998; Yee et al., 2010; Zhang et al., 2012) were included in schizophrenia, 16 studies (Adler et al., 1990a; Ancin et al., 2011; Cabranes et al., 2013; Carroll et al., 2008; Domjan et al., 2012; Franks et al., 1983; Hall et al., 2015a; Hall et al., 2014; Hall et al., 2008; Johannesen et al., 2013; Lijffijt et al., 2009; Martin et al., 2007a; Olincy and Martin, 2005; Patterson et al., 2009; Sanchez-Morla et al., 2008; Schulze et al., 
2007) were included in bipolar disorder, 10 studies in first-degree relatives (Clementz et al., $\underset{山}{\stackrel{\Upsilon}{山}} \quad$ 1998b; de Wilde et al., 2007a; Hall et al., 2008; Hong et al., 2008b; Louchart-de la Chapelle et al., 2005b; Myles-Worsley, 2002; Olincy et al., 2010; Schulze et al., 2007; Siegel et al., 1984) and 110 studies included healthy controls (Adler et al., 1990a; Adler et al., 2004; Adler et al., 1982; Adler et al., 1985; Adler et al., 1990b; Arnfred et al., 2003; Arnfred et al., 2004; Bak et al., 2014; Baker et al., 1987; Becker et al., 2004; Becker et al., 2011; Boutros et al., 1999; Boutros et al., 2009; Boutros et al., 2004; Braff et al., 2007; Brenner et al., 2009; BrockhausDumke et al., 2008a; Brockhaus-Dumke et al., 2008b; Cabranes et al., 2013; Carroll et al., 2008; Chen et al., 2011; Clementz and Blumenfeld, 2001; Clementz et al., 1997a; Clementz et al., 1997b, 1998a, b; Cullum et al., 1993; Dalecki et al., 2016; de Wilde et al., 2007a; Demily et al., 2016; Devrim-Ucok et al., 2008; Domjan et al., 2012; During et al., 2014; El-Kaim et al., 2015; Franks et al., 1983; Freedman et al., 1987; Fresan et al., 2007; Ghisolfi et al., 2006; Ghisolfi et al., 2004; Ghisolfi et al., 2002; Gjini et al., 2010; Gjini et al., 2011; Griffith and Freedman, 1995; Griffith et al., 1993; Hall et al., 2015a; Hall et al., 2014; Hall et al., 2008; Hong et al., 2004; Hong et al., 2008b; Hong et al., 2007; Hong et al., 2009; Hsieh et al., 2004; Hsieh et al., 2012; Jin et al., 1998; Johannesen et al., 2005; Johannesen et al., 2013; Judd et al., 1992; Kathmann and Engel, 1990; Keri et al., 2010; Kisley and Cornwell, 2006; Koike et al., 2005; Lijffijt et al., 2009; Louchart-de la Chapelle et al., 2005a; Louchart-de la Chapelle et al., 2005b; Lu et al., 2007; Martin et al., 2007a; Mazhari et al., 2011; Micoulaud-Franchi et al., 2015a; Micoulaud-Franchi et al., 2012; Micoulaud-Franchi et al., 2014; Moran et al., 2012; MylesWorsley, 2002; Nagamoto et al., 1989; Nagamoto et al., 1991; Neuhaus et al., 2014; Olincy et al., 2010; Olincy and Martin, 2005; Olincy et al., 2000; Oranje and Glenthoj, 2014; Price et al., 2006; Rentzsch et al., 2007; Ringel et al., 2004; Sanchez-Morla et al., 2008; Sanchez-Morla et al., 2013; Sanchez-Morla et al., 2009; Santos et al., 2010; Schulze et al., 2007; Shan et al., 2013; Siegel et al., 1984; Smith et al., 2010; Smith et al., 2013; Smucny et al., 2013; Song et al., 2014; Storozheva et al., 2016; Thoma et al., 2003; Thoma et al., 2008; Turetsky et al., 2009; Waldo et al., 1992; Waldo et al., 1988; Waldo et al., 1994; Waters et al., 2009; Williams et al., 2011; Wonodi et al., 2014; Yee et al., 1998; Yee et al., 2010; Zhang et al., 2012). Both study selection and data extraction were performed by two authors independently for both schizophrenia (IKA and MIA) and bipolar disorder (SZT and MIA), a third author (MHH) made final decisions for conflicts. First-degree relatives of both schizophrenia and bipolar disorder were pooled to obtain a sufficient number of studies for the meta-analysis. 
Figure 2. Flow-chart indicating the algorithm of literature search and study selection procedures
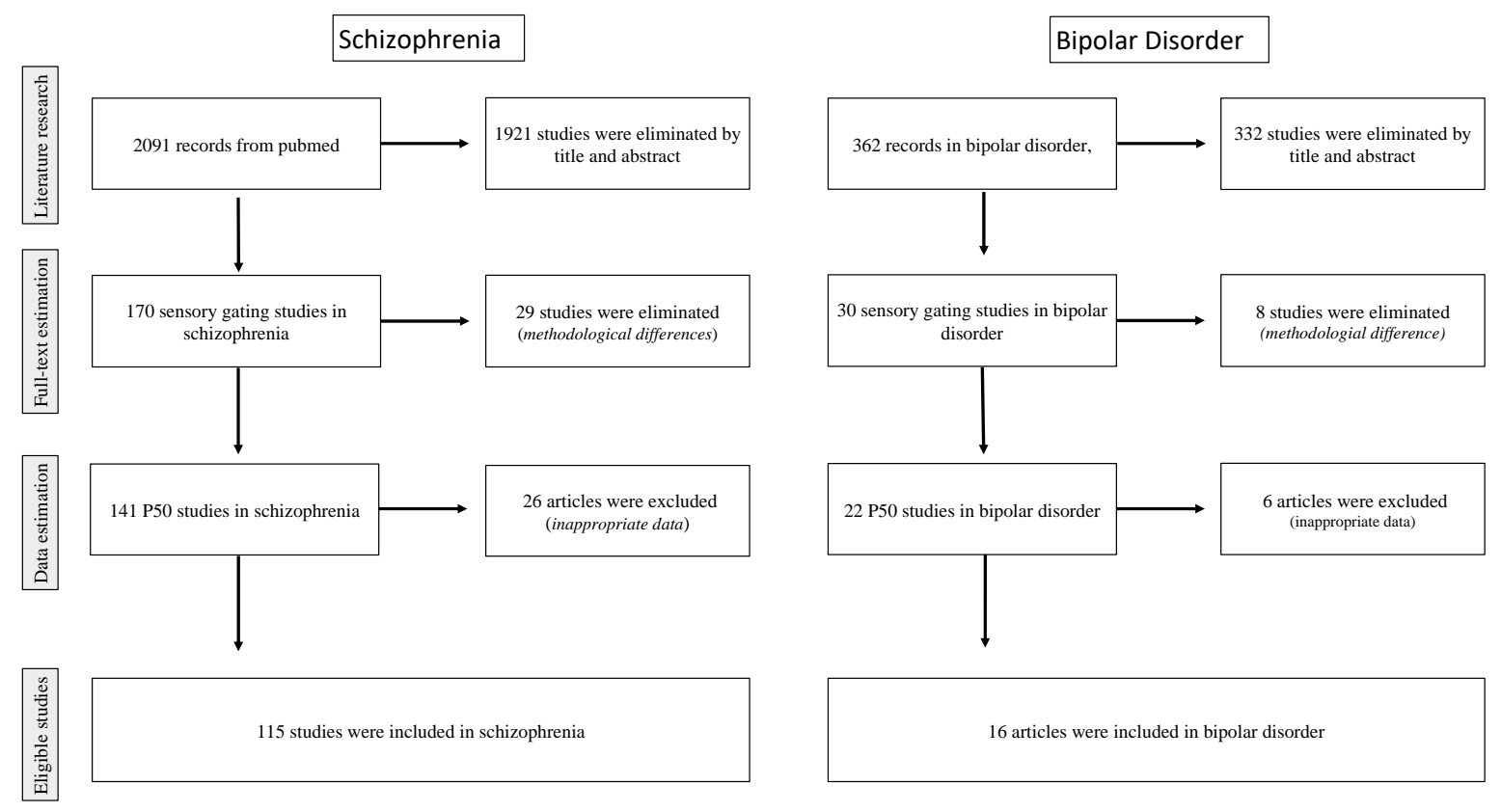

\subsection{Data extraction}

Means and standard deviations of the groups and sub-groups were extracted from all studies and independent effect sizes were obtained from each of the groups and sub-groups. Data were collected to form diagnostic groups, illness state subgroups, medication subgroups, and psychotic and non-psychotic groups in bipolar disorder.

\subsection{Outcome measures}

In a dual-click paradigm experiment, a set of stimuli consisting of click-pairs (two successive clicks [S1-conditioning stimulus and S2-test stimulus] with an interval of 500 milliseconds between the click-pairs) is presented to the subject and the reduction in the responses of the second stimulus (S2) of the pair is proposed to be the neurophysiological manifestation of the sensory gating function, see Figure 1 for an example. Variation of the two responses might be better monitored with a ratio of the two responses (S2/S1) or by the difference between the S1 and S2 amplitudes (S1-S2). (Hall et al., 2006) Lower S2/S1 ratio or larger S1-S2 difference reflect more sensory gating. S2/S1 ratio is a coherent measure of the change in the magnitude of the test stimulus in comparison to the conditioning stimulus. In particular, S2/S1 and amplitude of the S1 response were the most consistently reported measures in studies. 


\subsection{Data Analysis}

\section{Main grouping variables}

The following study groups were schizophrenia, bipolar disorder, healthy controls, first-degree relatives (diagnostic groups). Schizophrenia included two illness states: (chronic) schizophrenia and first episode psychosis. A period of time a person is experiencing psychotic symptoms for the first time in her life (and that episode should have lasted less than 6 months in time and no relapse into a new psychotic episode have occurred) is described as first-episode psychosis in the literature (de Wilde et al., 2007a). First-degree relatives include participants that are parents, siblings and offsprings of the patients with bipolar disorder and schizophrenia. Bipolar disorder included two illness states: remission and episodes (both mania and depression are labeled as episode). Studies that include first-degree relatives groups in both bipolar disorder (Hall et al., 2008; Schulze et al., 2007) and schizophrenia (Clementz et al., 1998b; de Wilde et al., 2007a; Hong et al., 2008b; Myles-Worsley, 2002; Olincy et al., 2010) were pooled as first-degree relatives. Healthy controls and first-degree relatives did not include subgroups. Medication states were medicated, medication-free, and medication naïve. Only the diagnostic group bipolar disorder had subgroups for psychotic symptoms (yes / no / not-specified).

\section{Statistical analyses}

All analyses were performed using Stata version 15 (StataCorp., 2017). In all meta-analysis commands, the random-effects option (a more conservative approach to deal with heterogeneous data across studies) was used. To study whether there were differences in the outcomes (S1 amplitude, S2 amplitude, S2/S1 ratio, S1-S2 difference) between the diagnostic groups, meta-regression analyses were performed using the Stata command 'metareg'; healthy controls were the reference category. Postestimation, schizophrenia was directly compared with bipolar disorder using an F-test (Stata command: 'test'). To illustrate these results, forest plots were generated, stratified by diagnostic group. In the above analyses, all available data were included. A small subgroup of included studies assessing both schizophrenia patients and bipolar disorder patients was used to perform within study comparisons (Figure 5).

To study the second, third and fourth aim, meta-regression analyses were performed, and forest plots were generated. To analyze the second aim, data were stratified by illness state subgroups (again healthy controls were the reference group). For the third aim, data were stratified by medication use and diagnostic group and for the fourth aim data were stratified by psychotic symptoms within the bipolar patient group. 
All analyses below were performed for each diagnostic group separately (schizophrenia, bipolar disorder, healthy controls, relatives). Influences of each single study on the meta-analysis results were examined using the 'metainf' command (how much does the outcome parameter change when omitting one study?). To assess publication bias, funnel plots were generated and Eggers tests were performed (Stata commands 'metafunnel' and 'metabias', respectively). Trim and fill analysis ('metatrim') was performed to estimate the effects of publication bias. To study whether there were differences in the outcomes (S1 amplitude, S2 amplitude, S2/S1 ratio, S1S2 difference) between the diagnostic groups, meta-regression analyses were performed using the Stata command 'metareg'; healthy controls were the reference category. The regression coefficients (B) present the difference between the patient group and the comparison group.

Stimulus duration, stimulus intensity (of stimulus 1 and 2, respectively), number of stimuli, illness state subgroup, medication status and psychotic symptoms were a priori selected as modifiers in the meta-analyses. In other words, these variables were hypothesized to be responsible for heterogeneity. First, these variables were added to the meta-regression, one at a time and all simultaneously. Subsequently, forest plots were generated after stratifying the data by modifiers (when continuous, recoded into quartiles) that were statistically significant in the meta-regression to find out whether this reduced heterogeneity. The modifiers, illness state subgroup and medication status, were excluded when analyzing healthy controls and firstdegree relatives. Psychotic and non-psychotic patients with bipolar disorder were also compared.

\section{Results}

\subsection{Characteristics of the Included Studies}

A total of 130 studies were included in the analyses. 115 studies (4932 patients) were schizophrenia reports, 16 studies (975 patients) had bipolar disorder groups, 10 first-degree relative groups (848 participants) and 110 studies (4127 subjects) included healthy controls. Table 1 presents numbers of studies and numbers of subjects included in each analysis. Eight studies included both schizophrenia (541 subjects) and bipolar disorder (581 subjects) groups (Cabranes et al., 2013; Domjan et al., 2012; Hall et al., 2015a; Hall et al., 2014; Johannesen et al., 2013; Martin et al., 2007a; Olincy and Martin, 2005; Patterson et al., 2009).

\subsection{Diagnostic Groups}

Meta-regression analysis showed that $\mathrm{S} 2 / \mathrm{S} 1$ ratio [B=26.18 (95\% CI: 21.20, 31.15), $\mathrm{p}<0.001]$ and S1-S2 difference $[\mathrm{B}=-0.63(95 \% \mathrm{CI}:-0.98,0.29), \mathrm{p}<0.001]$ were significantly different 
between schizophrenia patients and healthy controls. S2/S1 ratio [B= 22.52 (95\% CI: 12.86, 㐍 32:28), $\mathrm{p}<0.001]$ and $\mathrm{S} 1-\mathrm{S} 2$ difference $[\mathrm{B}=-0.94$ (95\% CI: -1.51, -0.37), $\mathrm{p}=0.002]$ were also significantly different in bipolar disorder than healthy controls (Table 1). Schizophrenia patients had smaller S1 [B= -0.45 (95\% CI: -0.90, -0.01), p=0.048] and larger S2 [B=0.51 (95\% CI: $0.29,0.74), \mathrm{p}<0.001]$ amplitudes than healthy controls. First-degree relatives had higher $\mathrm{S} 2 / \mathrm{S} 1$ ratio $[\mathrm{B}=13.91$ (95\% CI: 1.98, 25.83), $\mathrm{p}=0.022]$ compared to healthy controls (Table 1). Figure 3 and 4 are summarizing the forest-plots of all four outcomes. Full forest-plots are available in the supplementary material (Figure S1). When postestimation comparing bipolar disorder with schizophrenia, there were no differences in any of the four outcomes (Figure 5). Numbers in the within study meta-analyses were far smaller (Table 2). Results showed that S1 amplitudes were lower and S2/S1 ratio was higher in schizophrenia patients than in bipolar disorder patients, while there were no significant differences in the other two outcomes (Figure $5)$. 
Table 1. Numbers of study groups and participants per analysis and meta regression results: difference in outcome between any diagnostic group and healthy controls

\begin{tabular}{|c|c|c|c|c|c|}
\hline \multicolumn{3}{|c|}{ Between studies } & \multirow{2}{*}{$\begin{array}{c}\text { B } \\
-0.45\end{array}$} & \multirow{2}{*}{$\begin{array}{c}95 \% \\
\begin{array}{c}95 \% \\
\text { Confidence } \\
\text { Interval }\end{array} \\
-0.90,-0.003\end{array}$} & \multirow{2}{*}{$\begin{array}{c}\begin{array}{c}\text { p- } \\
\text { value }\end{array} \\
0.048\end{array}$} \\
\hline \multirow{15}{*}{ 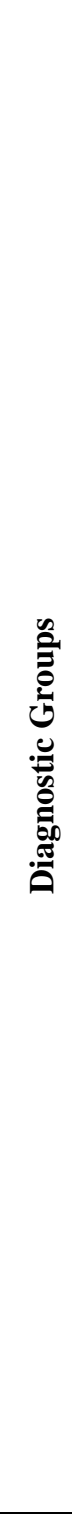 } & \multirow{3}{*}{$\begin{array}{l}\text { S1 Amplitude } \\
\text { (Figure 3-A) }\end{array}$} & $\begin{array}{l}\text { SZ (112 study groups, } 3833 \text { participants) vs } \\
\text { HCs (90 study groups, } 4383 \text { participants) }\end{array}$ & & & \\
\hline & & $\begin{array}{l}\text { BD (11 study groups, } 700 \text { participants) vs } \\
\text { HCs (90 study groups, } 4383 \text { participants) }\end{array}$ & -0.37 & $-1.36,0.63$ & 0.466 \\
\hline & & $\begin{array}{l}\text { FDR ( } 8 \text { study groups, } 725 \text { participants) vs } \\
\text { HCs (90 study groups, } 4383 \text { participants) }\end{array}$ & -0.87 & $-1.99,0.26$ & 0.132 \\
\hline & & $\begin{array}{l}\text { SZ (112 study groups, } 3833 \text { participants) vs } \\
\text { BD (11 study groups, } 700 \text { participants) }\end{array}$ & $F=0.03$ & $d f=1,221$ & $p=0.87$ \\
\hline & \multirow{4}{*}{$\begin{array}{l}\text { S2 Amplitude } \\
\text { (Figure 3-B) }\end{array}$} & $\begin{array}{l}\text { SZ (91 study groups, } 3461 \text { participants) vs } \\
\text { HCs (73 study groups, } 3250 \text { participants) }\end{array}$ & 0.51 & $0.29,0.74$ & $<0.001$ \\
\hline & & $\begin{array}{l}\text { BD (13 study groups, } 744 \text { participants) vs } \\
\text { HCs ( } 73 \text { study groups, } 3250 \text { participants) }\end{array}$ & 0.36 & $-0.06,0.79$ & 0.091 \\
\hline & & $\begin{array}{l}\text { FDR ( } 8 \text { study groups, } 769 \text { participants) vs } \\
\text { HCs ( } 73 \text { study groups, } 3250 \text { participants) }\end{array}$ & 0.30 & $-0.22,0.82$ & 0.260 \\
\hline & & $\begin{array}{l}\text { SZ (91 study groups, } 3461 \text { participants) vs BD } \\
\text { (13 study groups, } 744 \text { participants) }\end{array}$ & $F=0.50$ & $d f=1,185$ & $p=0.48$ \\
\hline & \multirow{3}{*}{$\begin{array}{l}\text { S2/S1 } \\
\text { (Figure 4-A) }\end{array}$} & $\begin{array}{l}\text { SZ (132 study groups, } 3666 \text { participants) vs } \\
\text { HCs (102 study groups, } 3464 \text { participants) }\end{array}$ & 26.18 & $21.20,31.15$ & $<0.001$ \\
\hline & & $\begin{array}{l}\text { BD (19 study groups, } 656 \text { participants) vs } \\
\text { HCs (102 study groups, } 3464 \text { participants) }\end{array}$ & 22.57 & $12.86,32.28$ & $<0.001$ \\
\hline & & $\begin{array}{l}\text { FDR (8 study groups, } 769 \text { participants) vs } \\
\text { HCs (102 study groups, } 3464 \text { participants) }\end{array}$ & 13.91 & $1.98,25.83$ & 0.022 \\
\hline & & $\begin{array}{l}\text { SZ (132 study groups, } 3666 \text { participants) vs } \\
\text { BD (19 study groups, } 656 \text { participants) }\end{array}$ & $F=0.54$ & $d f=1,263$ & $p=0.46$ \\
\hline & \multirow{3}{*}{$\begin{array}{l}\text { S1-S2 } \\
\text { (Figure 4-B) }\end{array}$} & $\begin{array}{l}\text { SZ (34 study groups, } 1476 \text { participants) vs } \\
\text { HCs (32 study groups, } 1620 \text { participants) }\end{array}$ & -0.63 & $-0.98,0.29$ & $<0.001$ \\
\hline & & $\begin{array}{l}\text { BD ( } 7 \text { study groups, } 226 \text { participants) vs } \\
\text { HCs ( } 32 \text { study groups, } 1620 \text { participants) }\end{array}$ & -0.94 & $-1.51,-0.37$ & 0.002 \\
\hline & & $\begin{array}{l}\text { FDR (6 study groups, } 612 \text { participants) vs } \\
\text { HCs ( } 32 \text { study groups, } 1620 \text { participants) }\end{array}$ & -0.31 & $1.61,2.10$ & 0.309 \\
\hline & & $\begin{array}{l}\text { SZ (34 study groups, } 1476 \text { participants) vs BD } \\
\text { (7 study groups, } 226 \text { participants) }\end{array}$ & $F=1.11$ & $d f=1,77$ & $p=0.29$ \\
\hline \multirow{5}{*}{ 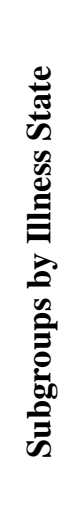 } & \multirow{5}{*}{$\begin{array}{l}\text { S1 Amplitude } \\
\text { (Figure 3-C) }\end{array}$} & $\begin{array}{l}\text { SZ (101 study groups, } 3416 \text { participants) vs } \\
\text { HCs (90 study groups, } 3250 \text { participants) }\end{array}$ & -0.46 & $-0.92,-0.01$ & 0.047 \\
\hline & & $\begin{array}{l}\text { FEP (11 study groups, } 368 \text { participants) vs } \\
\text { HCs (90 study groups, } 3250 \text { participants) }\end{array}$ & -0.33 & $-1.33,0.67$ & 0.514 \\
\hline & & $\begin{array}{l}\text { BD remission ( } 9 \text { study groups, } 606 \\
\text { participants) vs HCs ( } 90 \text { study groups, } 3614 \\
\text { participants) }\end{array}$ & -0.71 & $-1.78,0.37$ & 0.195 \\
\hline & & $\begin{array}{l}\text { BD episodes ( } 2 \text { study groups, } 104 \text { participants) } \\
\text { vs HCs ( } 90 \text { study groups, } 3614 \text { participants) }\end{array}$ & 1.29 & $-0.98,3.57$ & 0.264 \\
\hline & & $\begin{array}{l}\text { FDR ( } 8 \text { study groups, } 769 \text { participants) vs } \\
\text { HCs (90 study groups, } 3614 \text { participants) }\end{array}$ & -0.87 & $-1.99,0.26$ & 0.131 \\
\hline
\end{tabular}




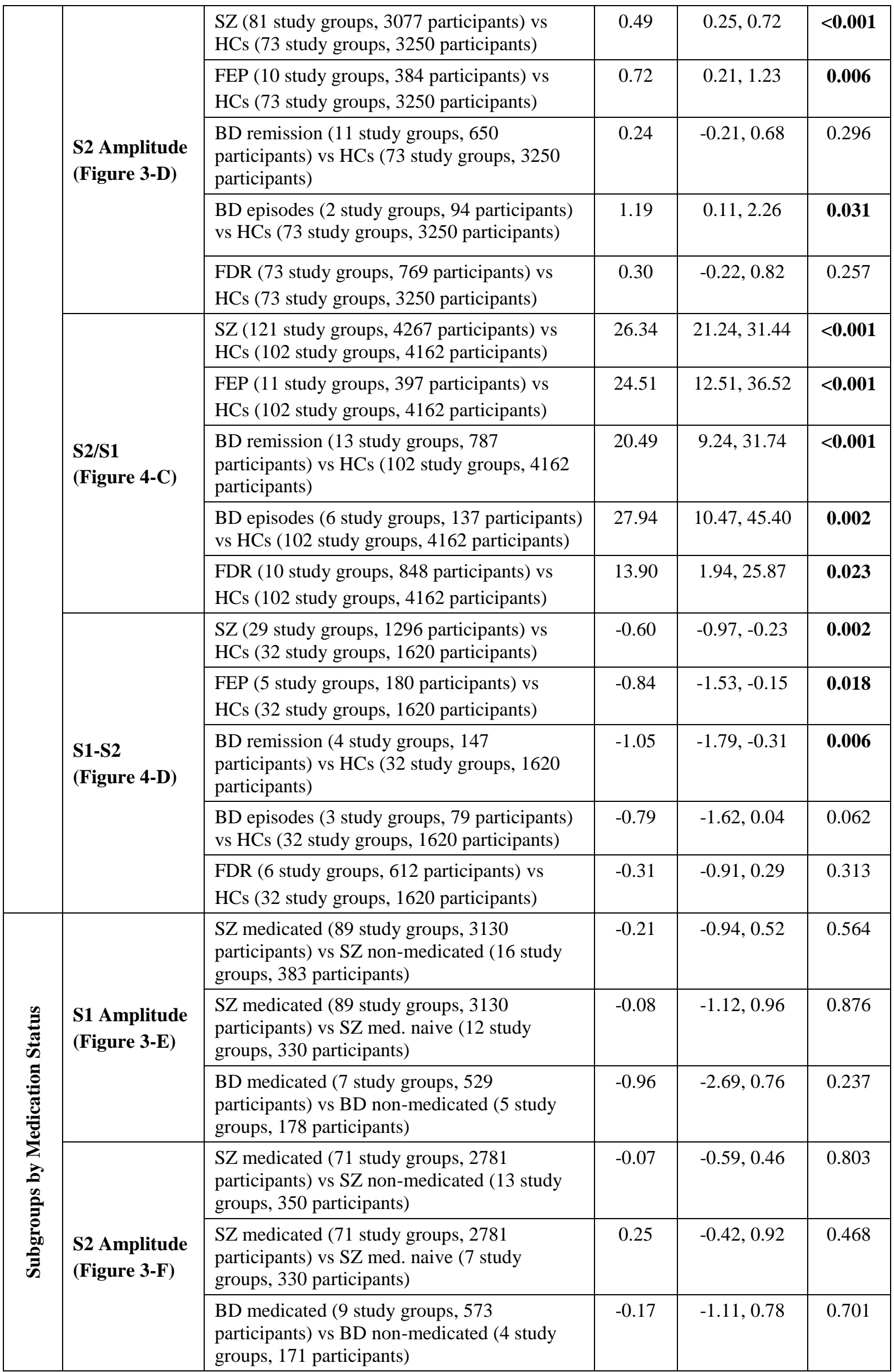




\begin{tabular}{|c|c|c|c|c|c|}
\hline & \multirow{3}{*}{$\begin{array}{l}\text { S2/S1 } \\
\text { (Figure 4-E) }\end{array}$} & $\begin{array}{l}\text { SZ medicated (109 study groups, } 3876 \\
\text { participants) vs SZ non-medicated ( } 16 \text { study } \\
\text { groups, } 459 \text { participants) }\end{array}$ & 11.87 & $0.76,22.97$ & 0.036 \\
\hline & & $\begin{array}{l}\text { SZ medicated (109 study groups, } 3876 \\
\text { participants) vs SZ med. naive ( } 7 \text { study } \\
\text { groups, } 330 \text { participants) }\end{array}$ & 6.10 & $-9.37,21.56$ & 0.437 \\
\hline & & $\begin{array}{l}\text { BD medicated (12study groups, } 634 \\
\text { participants) vs BD non-medicated ( } 7 \text { study } \\
\text { groups, } 290 \text { participants) }\end{array}$ & 13.00 & $-6.63,32.62$ & 0.180 \\
\hline & \multirow{3}{*}{$\begin{array}{l}\text { S1-S2 } \\
\text { (Figure 4-F) }\end{array}$} & $\begin{array}{l}\text { SZ medicated ( } 26 \text { study groups, } 1187 \\
\text { participants) vs SZ non-medicated ( } 4 \text { study } \\
\text { groups, } 125 \text { participants) }\end{array}$ & -0.77 & $-1.33,-0.21$ & 0.008 \\
\hline & & $\begin{array}{l}\text { SZ medicated ( } 26 \text { study groups, } 1187 \\
\text { participants) vs SZ med. naive (4 study } \\
\text { groups, } 164 \text { participants) }\end{array}$ & -0.21 & $-0.77,0.35$ & 0.454 \\
\hline & & $\begin{array}{l}\text { BD medicated ( } 2 \text { study groups, } 70 \\
\text { participants) vs BD non-medicated ( } 5 \text { study } \\
\text { groups, } 156 \text { participants) }\end{array}$ & -1.33 & $-2.40,-0.29$ & 0.022 \\
\hline \multirow{4}{*}{ 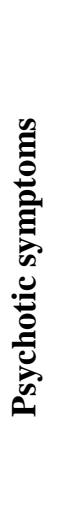 } & $\begin{array}{l}\text { S1 Amplitude } \\
\text { (Figure 3-G) }\end{array}$ & $\begin{array}{l}\text { BP without psychotic symptoms (1 study, } 30 \\
\text { participants) vs BD with psychotic symptoms } \\
\text { (5 study groups, } 303 \text { participants) }\end{array}$ & 1.00 & $-1.60,3.60$ & 0.38 \\
\hline & $\begin{array}{l}\text { S2 Amplitude } \\
\text { (Figure 3-H) }\end{array}$ & $\begin{array}{l}\text { BP without psychotic symptoms ( } 2 \text { study } \\
\text { groups, } 45 \text { participants) vs BD with psychotic } \\
\text { symptoms (6 study groups, } 332 \text { participants) }\end{array}$ & -0.15 & $-1.64,1.34$ & 0.82 \\
\hline & $\begin{array}{l}\text { S2/S1 } \\
\text { (Figure 4-G) }\end{array}$ & $\begin{array}{l}\text { BP without psychotic symptoms ( } 4 \text { study } \\
\text { groups, } 68 \text { participants) vs BD with psychotic } \\
\text { symptoms ( } 7 \text { study groups, } 345 \text { participants) }\end{array}$ & 10.7 & $-19.2,40.6$ & 0.46 \\
\hline & $\begin{array}{l}\text { S1-S2 } \\
\text { (Figure 4-H) }\end{array}$ & $\begin{array}{l}\text { BP without psychotic symptoms (1 study, } 16 \\
\text { participants) vs BD with psychotic symptoms } \\
\text { (4 study groups, } 138 \text { participants) }\end{array}$ & -0.35 & $-1.09,0.38$ & 0.23 \\
\hline
\end{tabular}

Table 2. Numbers of study groups and participants per analysis in the within study groups meta-analysis

\begin{tabular}{|c|c|c|}
\hline \multirow{4}{*}{ 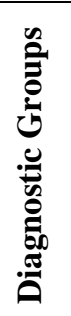 } & $\begin{array}{l}\text { S1 Amplitude } \\
\text { (Figure 5-A) }\end{array}$ & 6 study groups, $439 \mathrm{SZ}$ and $400 \mathrm{BD}$ participants \\
\hline & $\begin{array}{l}\text { S2 Amplitude } \\
\text { (Figure 5-B) }\end{array}$ & 7 study groups, $451 \mathrm{SZ}$ and $415 \mathrm{BD}$ participants \\
\hline & $\begin{array}{l}\text { S2/S1 } \\
\text { (Figure 5-C) }\end{array}$ & 9 study groups, $574 \mathrm{SZ}$ and $545 \mathrm{BD}$ participants \\
\hline & $\begin{array}{l}\text { S1-S2 } \\
\text { (Figure 5-D) }\end{array}$ & 2 study groups, $70 \mathrm{SZ}$ and $70 \mathrm{BD}$ participants \\
\hline
\end{tabular}

Sz: Schizophrenia, BD: Bipolar Disorder, HCs: healthy controls, FDR: First-Degree

Relatives, FEP: First episode psychosis. B: Regression coefficient 
Figure 3. Meta-analysis results of $\mathrm{S} 1$ and $\mathrm{S} 2$ responses. Representative averaged effect sizes

and confidence intervals are presented.

\section{S1 Amplitudes}

Diagnostic Groups
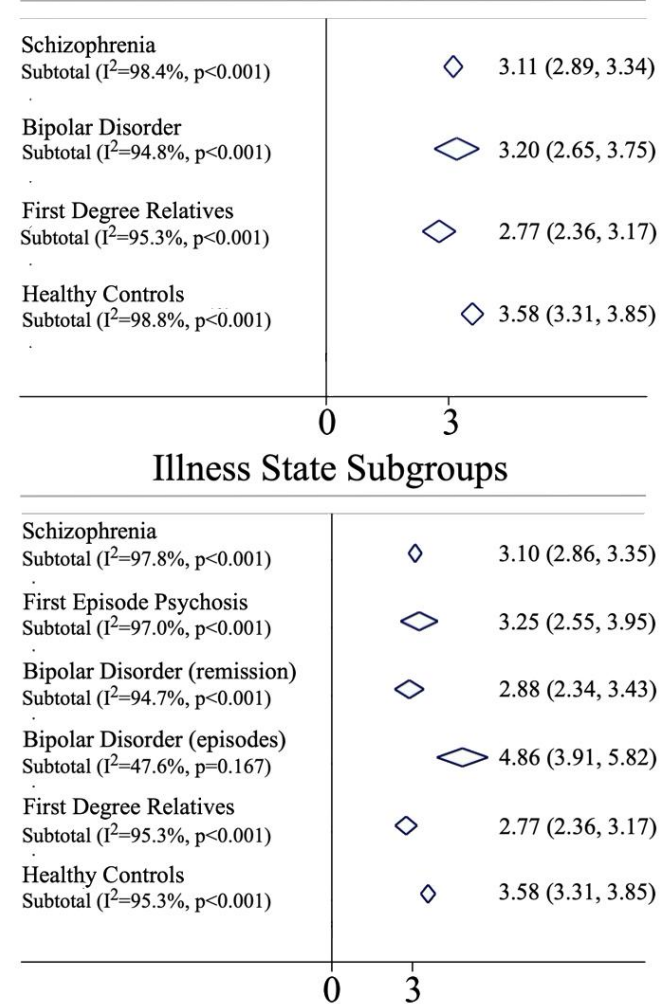

\section{Medication Status Subgroups}
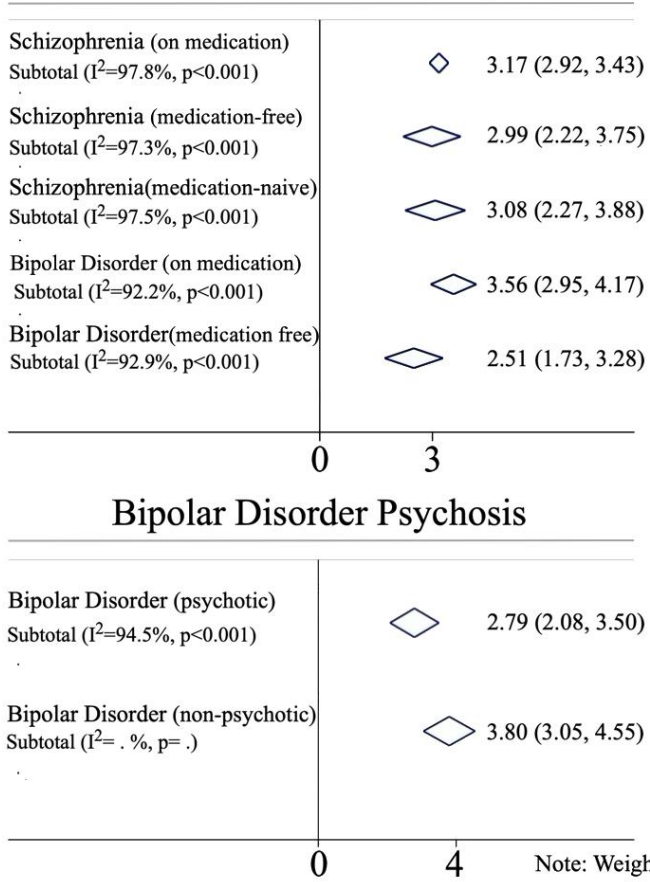

\section{S2 Amplitudes}

Diagnostic Groups

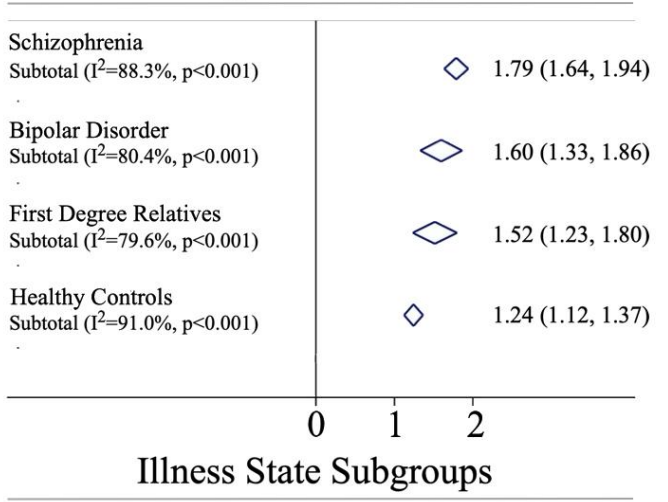

\begin{tabular}{|c|c|c|}
\hline $\begin{array}{l}\text { Schizophrenia } \\
\text { Subtotal }\left(I^{2}=85.4 \%, p<0.001\right)\end{array}$ & $\Delta$ & $1.74(1.59,1.89)$ \\
\hline $\begin{array}{l}\text { First Episode Psychosis } \\
\text { Subtotal }\left(\mathrm{I}^{2}=95.3 \%, \mathrm{p}<0.001\right)\end{array}$ & & $2.16(1.44,2.87)$ \\
\hline $\begin{array}{l}\text { Bipolar Disorder (remission) } \\
\text { Subtotal }\left(\mathrm{I}^{2}=77.8 \%, \mathrm{p}<0.001\right)\end{array}$ & $\diamond$ & $1.48(1.22,1.73)$ \\
\hline $\begin{array}{l}\text { Bipolar Disorder (episodes) } \\
\text { Subtotal }\left(\mathrm{I}^{2}=79.9 \%, \mathrm{p}=0.167\right)\end{array}$ & & $2.49(1.29,3.69)$ \\
\hline $\begin{array}{l}\text { First Degree Relatives } \\
\text { Subtotal }\left(\mathrm{I}^{2}=79.6 \%, \mathrm{p}<0.001\right)\end{array}$ & $\diamond$ & $1.52(1.23,1.80)$ \\
\hline $\begin{array}{l}\text { Healthy Controls } \\
\text { Subtotal }\left(\mathrm{I}^{2}=91.0 \%, \mathrm{p}<0.001\right)\end{array}$ & 0 & $1.24(1.12,1.37)$ \\
\hline
\end{tabular}

Medication Status Subgroups

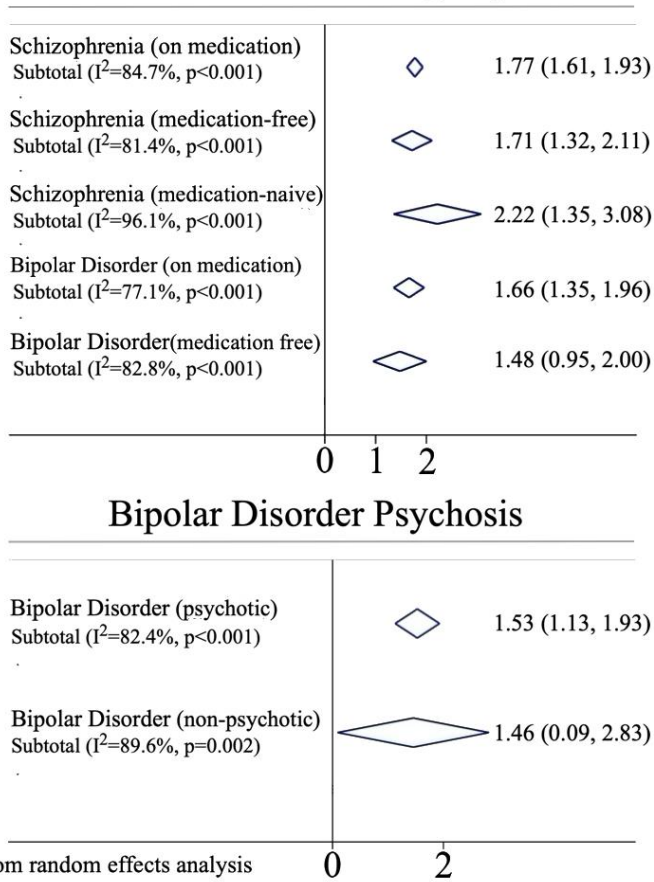


Figure 4. Meta-analysis results of S2/S1 ratio and S1 - S2 difference. Representative averaged effect sizes and confidence intervals are presented. For details, please see Figure S1.

\section{S2/S1 Ratio}

Diagnostic Groups

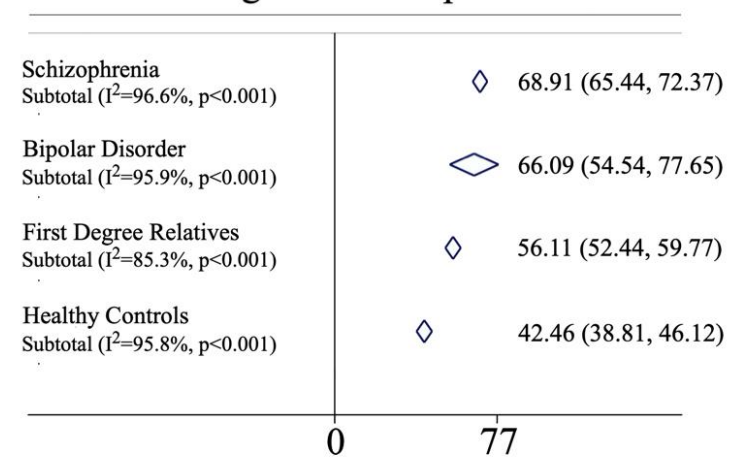

Illness State Subgroups

\begin{tabular}{|c|c|c|}
\hline $\begin{array}{l}\text { Schizophrenia } \\
\text { Subtotal }\left(\mathrm{I}^{2}=94.4 \%, \mathrm{p}<0.001\right)\end{array}$ & $\Delta$ & $68.70(65.61,71.79)$ \\
\hline $\begin{array}{l}\text { First Episode Psychosis } \\
\text { Subtotal }\left(\mathrm{I}^{2}=97.0 \%, \mathrm{p}<0.001\right)\end{array}$ & $\infty$ & $68.21(51.27,85.15)$ \\
\hline $\begin{array}{l}\text { Bipolar Disorder (remission) } \\
\text { Subtotal }\left(\mathrm{I}^{2}=95.6 \%, \mathrm{p}<0.001\right)\end{array}$ & $<$ & $63.32(50.85,75.80)$ \\
\hline $\begin{array}{l}\text { Bipolar Disorder (episodes) } \\
\text { Subtotal }\left(\mathrm{I}^{2}=94.2 \%, \mathrm{p}<0.001\right)\end{array}$ & & $73.45(48.51,98.39)$ \\
\hline $\begin{array}{l}\text { First Degree Relatives } \\
\text { Subtotal }\left(\mathrm{I}^{2}=85.3 \%, \mathrm{p}<0.001\right)\end{array}$ & 0 & $56.22(52.44,59.77)$ \\
\hline $\begin{array}{l}\text { Healthy Controls } \\
\text { Subtotal }\left(\mathrm{I}^{2}=98.5 \%, \mathrm{p}<0.001\right)\end{array}$ & 0 & $42.46(38.81,46.12)$ \\
\hline
\end{tabular}

\section{Medication Status Subgroups}

\begin{tabular}{|c|c|c|}
\hline $\begin{array}{l}\text { Schizophrenia (on medication) } \\
\text { Subtotal }\left(I^{2}=94.2 \%, p<0.001\right)\end{array}$ & $\diamond$ & $66.47(63.25,69.68)$ \\
\hline $\begin{array}{l}\text { Schizophrenia (medication-free) } \\
\text { Subtotal }\left(\mathrm{I}^{2}=89.2 \%, \mathrm{p}<0.001\right)\end{array}$ & $\diamond$ & $78.57(70.13,87.01)$ \\
\hline $\begin{array}{l}\text { Schizophrenia(medication-naive) } \\
\text { Subtotal }\left(\mathrm{I}^{2}=98.0 \%, \mathrm{p}<0.001\right)\end{array}$ & & $74.25(47.97,100.56)$ \\
\hline $\begin{array}{l}\text { Bipolar Disorder (on medication) } \\
\text { Subtotal }\left(\mathrm{I}^{2}=97.3 \%, \mathrm{p}<0.001\right)\end{array}$ & & $61.23(45.79,76.67)$ \\
\hline $\begin{array}{l}\text { Bipolar Disorder(medication free) } \\
\text { Subtotal }\left(\mathrm{I}^{2}=40.4 \%, \mathrm{p}=0.122\right)\end{array}$ & $\diamond$ & $69.42(60.88,77.95)$ \\
\hline
\end{tabular}

Bipolar Disorder Psychosis

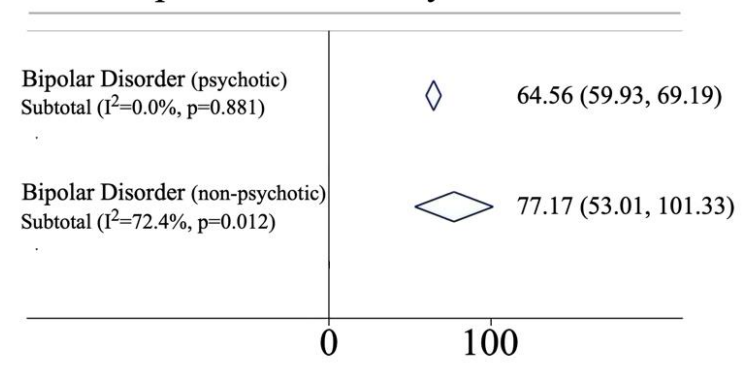

S1-S2 Difference

Diagnostic Groups

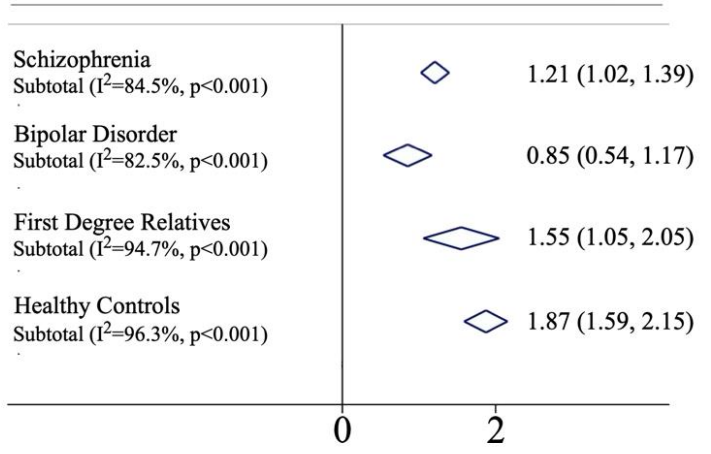

Illness State Subgroups

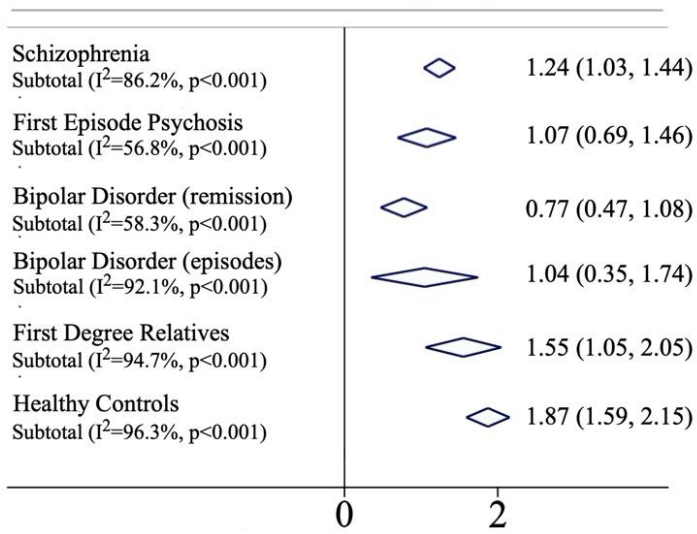

Medication Status Subgroups

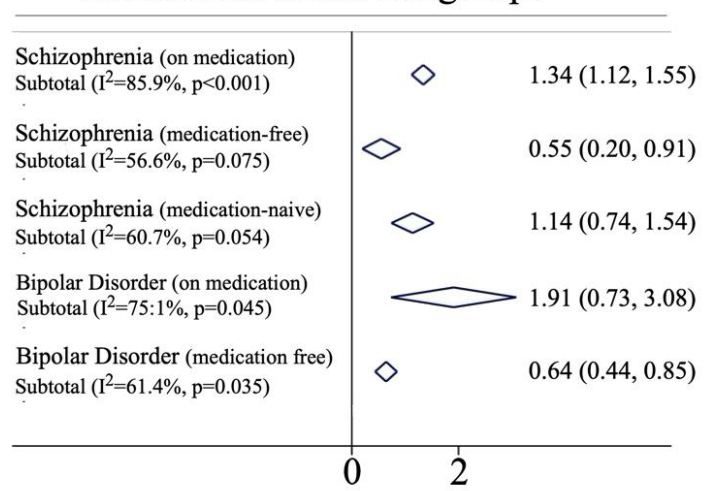

Bipolar Disorder Psychosis

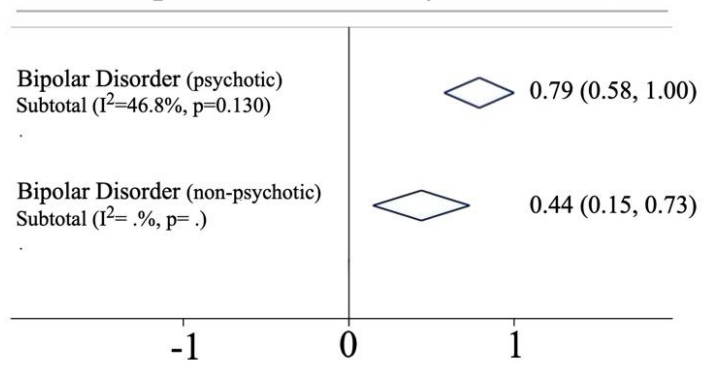


Figure 5. Meta-analysis results of the study groups comparing schizophrenia and bipolar disorder (e.g. when in Hall et al. 2015 S1 amplitude is lower in SZ than in BD SMD is below 0)
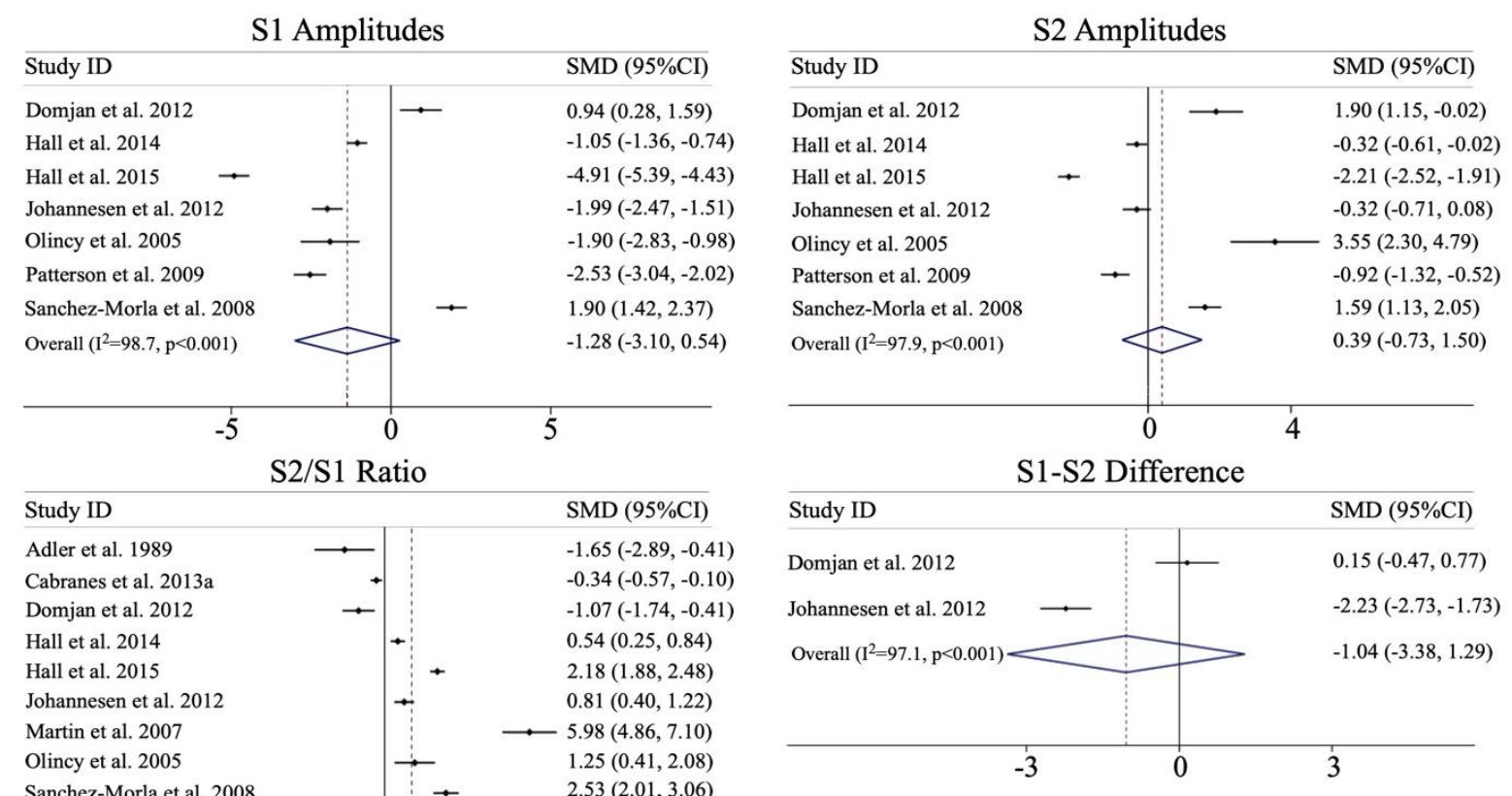

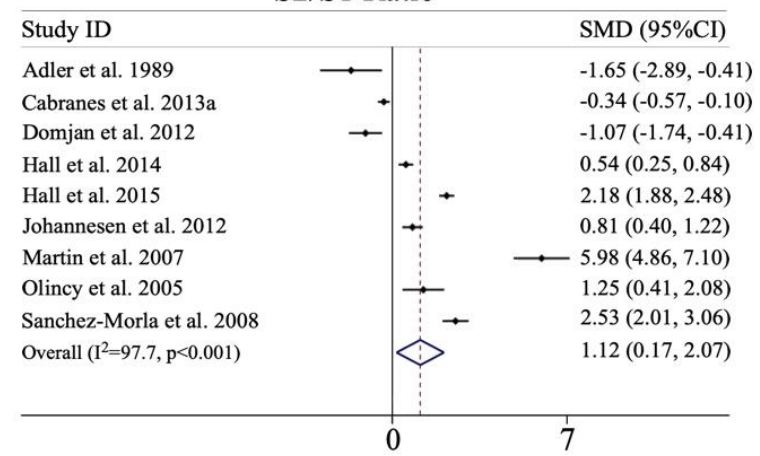

Note: Weights are from random effects analysis

\subsection{Robustness of results and publication bias}

Omitting single studies did not change the results of the forest-plots (figures available upon request). Egger Tests in schizophrenia patient groups and healthy controls did indicate publication bias for most outcomes, while Egger Tests in bipolar disorder groups did not show publication bias. Schizophrenia and healthy control funnel plots included far more studies and funnel plots were in agreement with the Egger test results showing a gap at the place of smallstudy-null-findings in all funnel-plots. Despite this, in S1 amplitudes trim and fill did not add extra studies (Figure S3). In most other outcomes, trim and fill did add extra studies. When trim and fill added studies, the difference decreased but remained statistically significant $(\mathrm{p}<0.001$, Figure S3). 


\subsection{Heterogeneity and modifiers}

Heterogeneity (between study results) was large with I-square ranging between $79.6 \%$ and $100 \%$ in the full samples. Of the a priori selected modifiers only a few were statistically significant. For example, stimulus duration $(\mathrm{p}=0.024)$ and number of stimuli $(\mathrm{p}=0.030)$ were modifiers for S1 amplitudes and number of stimuli $(\mathrm{p}=0.060)$ was a trend level modifier for S2 amplitudes in schizophrenia (Table S2) and illness state subgroup ( $\mathrm{p}=0.040$ ) was a modifier in bipolar disorder. When stratifying by a modifier, heterogeneity remained large in all strata (Table S2) with the exception of a few strata with a limited number of studies.

In healthy controls, there were no modifiers and in first-degree relatives, modifiers could not be analyzed because data were not available.

\subsection{IIIness State Subgroups}

The results are presented in Table 1, Figure 3 and Figure 4. In schizophrenia, S1 amplitudes $[\mathrm{B}=-0.46$ (95\% CI: $-0.92,-0.01), \mathrm{p}=0.047]$ and $\mathrm{S} 1-\mathrm{S} 2$ difference $[\mathrm{B}=-0.60$ (95\% CI: -0.97 , 0.23), $\mathrm{p}=0.002]$ were lower and $\mathrm{S} 2$ amplitudes $[\mathrm{B}=0.49$ (95\% CI: $0.25,0.72), \mathrm{p}<0.001]$ and S2/S1 ratio $[\mathrm{B}=26.34$ (95\% CI: 21.24, 31.44), $\mathrm{p}<0.001]$ compared with healthy controls. In first episode psychosis, $\mathrm{S} 1-\mathrm{S} 2$ difference $[\mathrm{B}=-0.84$ (95\% CI: $-1.53,-0.15), \mathrm{p}=0.018]$ were lower and $\mathrm{S} 2$ amplitudes $[\mathrm{B}=0.72(95 \% \mathrm{CI}: 0.21,1.23), \mathrm{p}=0.006]$ and $\mathrm{S} 2 / \mathrm{S} 1$ ratio $[\mathrm{B}=24.51(95 \%$ CI: 12.51, 36.52), $\mathrm{p}<0.001]$ compared with healthy controls Of all these, only the difference between first episode and healthy controls was not statistically significant.

In remission phase of bipolar disorder, S2/S1 ratio was higher [B= 20.49 (95\% CI: 9.24, 31.74), $\mathrm{p}<0.001]$ and $\mathrm{S} 1-\mathrm{S} 2$ difference was lower $[\mathrm{B}=-1.05$ (95\% CI: $-1.79,-0.31), \mathrm{p}=0.006]$ in comparison with healthy controls. Whereas in episodes of bipolar disorder, $\mathrm{S} 2$ amplitudes $[\mathrm{B}=$ 1.19 (95\% CI: 0.11, 2.26), $\mathrm{p}=0.031]$ and $\mathrm{S} 2 / \mathrm{S} 1$ ratio $[\mathrm{B}=27.94$ (95\% CI: 10.47, 45.40), $\mathrm{p}=0.002]$ were greater compared with healthy controls. There was difference between episodes and remission in bipolar disorder because of the differences in S1 (slightly enhanced in episodes, slightly decreased in remission) and S2 amplitudes between remission and episodes. In first-degree relatives, only effect size of $\mathrm{S} 2 / \mathrm{S} 1$ ratio $[\mathrm{B}=13.90$ (95\% CI: $1.94,25.87)$, $\mathrm{p}=0.023$ ] was significantly greater than healthy controls.

\subsection{Medication Subgroups}

Results are presented in the Table 1 and Figures 3 and 4. In schizophrenia, compared with medication-free patients medicated patients had higher S2/S1 ratio [B=11.87 (95\% CI: 0.76, 
22.97), $\mathrm{p}=0.036]$ and lower $\mathrm{S} 1-\mathrm{S} 2$ difference $[\mathrm{B}=-0.77$ (95\% CI: $-1.33,-0.21), \mathrm{p}=0.008]$. In bipolar disorder, S1-S2 differences were higher [B= -1.33 (95\% CI: $-2.40,-0.29), \mathrm{p}=0.022]$ in patients on medication in comparison with medication-free subjects.

\subsection{Psychotic symptoms in bipolar disorder}

In bipolar disorder, there were no significant differences in any of the outcomes between patients without and with psychotic symptoms (Table 1, Figure 2 and Figure 3).

\section{Discussion}

This study yielded several insights into the sensory gating alteration in schizophrenia and bipolar disorder. First, P50 sensory gating measured by dual-click paradigm is altered in both bipolar disorder and schizophrenia. Second, in line with the literature (Franks et al., 1983), we found that bipolar disorder patients, either in episodes or euthymic phases, exhibited P50 sensory gating alterations and the corresponding effect sizes were larger in episodes of bipolar disorder. In other words, sensory gating alteration in patients with bipolar disorder becomes more prominent during exacerbation phases of bipolar disorder. Third, P50 sensory gating alterations were similarly present in both first-episode psychosis patients and schizophrenia patients. Fourth, sensory gating alteration of first-degree relatives was at intermediate level between healthy controls and patients. Fifth, patient groups on medications had lower effect sizes in P50 sensory gating and medications seem to improve sensory gating ability in both schizophrenia and bipolar disorder. Finally, comparison between schizophrenia and bipolar disorder showed that schizophrenia showed greater P50 sensory gating alterations than bipolar disorder.

P50 sensory gating is altered in both schizophrenia and bipolar disorder; and our recent results confirmed this finding consistent with prior meta-reports (Bramon et al., 2004; Chang et al., 2011; Cheng et al., 2016; de Wilde et al., 2007b). S2/S1 sensory gating ratio was the main measure of sensory gating and both schizophrenia (ranging between 0.93-1.56) and bipolar disorder ( 0.85 for psychotic bipolar disorder, 0.59 for non-psychotic bipolar disorder $)$ had high effect sizes in the meta-analyses. Consistently, schizophrenia had higher effect sizes than bipolar disorder in all measures in our study. Alterions of inhibitory mechanisms may underlie sensory gating alterations in schizophrenia and bipolar disorder (Cross-Disorder Group of the Psychiatric Genomics, 2013). The study results were more consistent in schizophrenia. P50 sensory gating alterations may differentiate individuals with and without schizophrenia better than those with bipolar disorder. The level of P50 sensory gating ratio (S2/S1) alteration in 
first-degree relatives $[56.11(52.44,59.77)]$ were higher than healthy subjects [42.46 (38.81, 46.12)] but lower than schizophrenia [68.91 (65.44, 72.37)] schizophrenia and bipolar disorder [66.09 (54.54, 77.65)] and significantly higher than healthy controls [B=13.91, (95\%CI: 1.98, 25.83)] in this study, consistent with the literature (de Wilde et al., 2007b; Earls et al., 2016; Hall et al., 2007b; Hall et al., 2008; Myles-Worsley et al., 2004). Although first-degree relatives do not experience psychiatric symptoms, partial inheritance of the traits related with cognitive mechanisms may participate in veiled alterations of brain function. Deviation of the brain function might still remain within the variance limits of normal ranges or a mixture of normal and abnormal outcomes may alter the results slightly in first-degree relatives (Freedman et al., 1997; Greenwood et al., 2016). On the other hand, in an estimation of P50 sensory gating, P300 and mismatch negativity in first degree relatives of schizophrenia patients, P50 sensory gating had highest effect sizes and most consistently influenced measure in first-degree relatives of schizophrenia patients (Earls et al., 2016).

Our meta-analysis results indicate that P50 sensory gating alterations are both trait and state dependent markers. P50 sensory gating alterations do not progress with time in schizophrenia, remains stable during remission periods and deteriorate during acute exacerbation periods in bipolar disorder (Potter et al., 2006). This pattern is comparable with the lifetime course of neurocognitive alteration in schizophrenia and bipolar disorder (Lewandowski et al., 2011). Neurocognitive alterations that appear with premorbid (Trotta et al., 2015), prodromal (Hawkins et al., 2008) or first-episode phases (Hill et al., 2001; Hoff et al., 1998; Zanelli et al., 2010), remain stable over time (Irani et al., 2011) and deteriorate with symptomatic exacerbations (Bortolato et al., 2015; Milev et al., 2005) in schizophrenia. However, some cognitive domains are altered across all phases (working memory, attention, cognitive control) or some domains (auditory perception, verbal fluency) are disturbed in early phases of the disease (Kelly et al., 2018). In bipolar disorder, neurocognitive deficits become observable with first episode (Hirayasu et al., 1998; Zanelli et al., 2010), alleviate in remission periods (Torres et al., 2014) and effect sizes of neurocognitive alterations increase in episodes (Kurtz and Gerraty, 2009; Martinez-Aran et al., 2004) or with chronicity of the symptoms (de Almeida Rocca et al., 2008; Frangou et al., 2005). In addition, psychosis is a deteriorating symptom dimension for bipolar disorder. A recent meta-analysis revealed that psychotic patients had higher effect sizes in P50 sensory gating than non-psychotic patients with bipolar disorder (Cheng et al., 2016). Since inhibitory mechanisms of sensory gating provide resistance to interference and early cognitive inhibition at the encoding stage, we hypothesize that similar 
mechanisms may disturb both neurocognitive and P50 sensory gating alterations in 㐍 schizophrenia and bipolar disorder (Jones et al., 2016; Potter et al., 2006).

Illness course effects have been examined in schizophrenia with other paradigms in EEG. For example, Mismatch Negativity (MMN) studies show that chronic patients with schizophrenia had greater alteration than first-episode psychosis patients (Erickson et al., 2016). Auditory P300 measures could discriminate patients with schizophrenia, their first-degree relatives and healthy controls (Bramon et al., 2004; Earls et al., 2016; Niznikiewicz, 2019) and transition to psychosis was predicted by auditory P300 abnormalities in ultra-high-risk individuals (Tang et al., 2019). The trajectory of auditory P300 deterioration was corelated with disease conversion to psychosis among ultra-high-risk individuals (O'Donnell et al., 1995; Oribe et al., 2015). According to our results, auditory P50 sensory gating differs from auditory MMN and P300 ERPs, such that there is only slight difference between first-episode psychosis and schizophrenia, which did not reach statistical significance. Great variation in first-episode psychosis may have led this result. Larger samples and longitudinal studies are needed to determine potential changes of auditory P50 sensory gating in the course of psychosis.

In this meta-analysis, we were able to stratify patients according to medication status and compared medication subgroups. We found that medicated patient groups had better auditory P50 sensory gating compared to medication-free patients and interestingly that medication improve sensory gating function but did not normalize it. Several hypotheses have been tested in terms of neurotransmitters for P50 sensory gating. Nicotinic (Aidelbaum et al., 2018; Freedman, 2014), serotonergic (Mann et al., 2008; Oranje et al., 2011), dopaminergic (Adler et al., 2004; Devrim-Ucok et al., 2008; Oranje et al., 2013), noradrenergic (Adler et al., 1994; Witten et al., 2016) neurotransmission have been found to influence P50 sensory gating. Psychotropic medications alter neurophysiology with several mechanisms (Atagun, 2016; Atagun et al., 2015a; Vita et al., 2015; Yoshimura et al., 2007). Lithium amplifies event related oscillations in patients with bipolar disorder (Atagun et al., 2015a; Tan et al., 2016) and enhances sensory gating by stimulating norepinephrine and GABAergic neurotransmission in mice models (O'Neill et al., 2003). Two distinct mechanisms have been observed in enhancement of P50 sensory gating ratio by medications. One mechanism is amplification of evoked responses and the other mechanism is suppression of S2 response by supporting inhibitory mechanisms (Freedman, 2014). Stimulation of nicotinic alfa-7 receptors provide reduction in S2 amplitude and S2/S1 ratio (Adler et al., 1998; Aidelbaum et al., 2018) and clozapine may enhance sensory gating ratio by enhancing S1 amplitude (Nagamoto et al., 1996; 
Nagamoto et al., 1999). Globally medications seem to enhance S1 amplitudes prominently and S2 amplitudes slightly. Since P50 sensory gating is associated with exacerbation of symptoms, medications may have direct and indirect influences on P50 sensory gating measures in schizophrenia and bipolar disorder.

Although confounded by several factors including patients and medication combinations, a number of studies investigated medication effects on P50 sensory gating in schizophrenia and bipolar disorder. Various studies have reported that atypical antipsychotics improve (Adler et al., 2004; Csomor et al., 2014; de Wilde et al., 2007b; Devrim-Ucok et al., 2008; Holstein et al., 2011; Light et al., 2000; Oranje et al., 2013) or do not alter (Arango et al., 2003; Becker et al., 2004; During et al., 2014; Hong et al., 2009; Sanchez-Morla et al., 2009) P50 sensory gating. An early meta-analysis reported no significant effect of antipsychotics on pooled standardized effect sizes in schizophrenia (Bramon et al., 2004), however many studies are published afterwards. Medication may have differential effects, for example antipsychotics improve P50 sensory gating in people with sensory gating alterations, whereas do not affect P50 sensory gating ratio in people with normal P50 sensory gating (Csomor et al., 2009; Oranje et al., 2002; Ucar et al., 2012). These results indicate a global improvement in S2/S1 ratio and S1 - S2 difference in medicated patients compared to medication-free patients in schizophrenia and bipolar disorder. It has been suggested that medications can alleviate the findings of functional or structural neuroimaging studies (Hafeman et al., 2012), medication results of this study are consistent with this concept.

These results are limited with publication bias and heterogeneity and should therefore be interpreted cautiously. Clinical state variations between studies may account for the heterogeneous results, since P50 sensory gating ratio had correlation with clinical rating scales in studies (Potter et al., 2006). Clinical heterogeneity might be related with age at onset, illness duration, psychosis in bipolar disorder, comorbidities and substance use disorders. Variable results may also be related with variable responses to medications (de Wilde et al., 2007b; Patterson et al., 2008). Clinical characteristics such as age at onset, duration of the illness and cumulative effects of number of episodes could not be controlled in this study, since subjectby-subject data was not available. Differences in methodological applications between laboratories may have caused variable results and heterogeneity (Johannesen et al., 2013; Patterson et al., 2008). A meta-analysis have reported that methodological differences such as stimulus intensity or filter settings are influential factors for study results (de Wilde et al., 2007b). Furthermore, test-retest reliability of P50 sensory gating ratio is controversial, some 
authors reported low (Rentzsch et al., 2008) or high (Hall et al., 2006; Sandman and Patterson, 2000) reliability for P50 sensory gating ratio. Since the dual-click paradigm is a passive listening task, physical properties of the stimuli are highly critical. The majority of the studies have been performed with similar analysis methods, although there were differences between studies in terms of paradigms. However, it is proposed that P50 sensory gating studies may also be contaminated by the analysis procedures even when using the same analysis method. For instance, analyses might be confounded by the several components within the designated time range. It has been shown that selection of the S2 component significantly changes the P50 analysis results (Clementz et al., 1997b). Therefore, heterogeneity is possibly related with clinical characteristics of the patients, paradigms, EEG recordings and data analysis procedures. The stimulus characteristics (i.e., the number of stimuli, the duration of stimuli, and the intensity of stimuli) were significant modifiers for S1 amplitudes in this study. Stimulus sound intensity was found a significant modifier in a meta-analysis (de Wilde et al., 2007b), but two other meta-analyses showed no effect for the stimulus sound intensity (Chang et al., 2011; Patterson et al., 2008). Moreover, two meta-analyses reported that data analysis methods and research centers influenced the study results, and thus methodological differences may explain a proportion of the heterogeneity (Chang et al., 2011; Patterson et al., 2008). Physical properties of the stimuli are associated with $\mathrm{S} 1$ and $\mathrm{S} 2$ responses and thus are critically important, in particular, if the influence is different between S1 and S2.

To conclude, this meta-analysis examined the current literature of P50 sensory gating in schizophrenia and bipolar disorder. In addition, illness state and medication effects were also assessed. Findings showed that symptomatic exacerbations deteriorate, and medications alleviate alterations in the sensory gating mechanism. First-degree relatives had intermediate level sensory gating alteration between patients and healthy controls. Overall, this study suggests that P50 sensory gating alteration is a robust index in schizophrenia and affective psychosis spectrum disorders, and medications, to a small degree, seem to alleviate P50 sensory gating alteration. Although several studies assessed specific mechanisms of psychotropic medications, this meta-analysis presents an overall picture of global medication effect. Future studies with specific designs may systematically investigate potential effects of clinical dimensions and medications on P50 sensory gating.

Funding: None

Conflict of Interest: None

Acknowledgement: None 


\section{References}

1974. A glossary of terms most commonly used by clinical electroencephalographers. Electroencephalogr Clin Neurophysiol. 37 (5), 538-548.

Adler, L.E., Cawthra, E.M., Donovan, K.A., Harris, J.G., Nagamoto, H.T., Olincy, A., Waldo, M.C., 2005. Improved p50 auditory gating with ondansetron in medicated schizophrenia patients. Am J Psychiatry. 162 (2), 386-388.

Adler, L.E., Gerhardt, G.A., Franks, R., Baker, N., Nagamoto, H., Drebing, C., Freedman, R., 1990a. Sensory physiology and catecholamines in schizophrenia and mania. Psychiatry Res. 31 (3), 297-309.

Adler, L.E., Hoffer, L., Nagamoto, H.T., Waldo, M.C., Kisley, M.A., Giffith, J.M., 1994. Yohimbine impairs P50 auditory sensory gating in normal subjects. Neuropsychopharmacology. 10 (4), 249257.

Adler, L.E., Olincy, A., Cawthra, E.M., McRae, K.A., Harris, J.G., Nagamoto, H.T., Waldo, M.C., Hall, M.H., Bowles, A., Woodward, L., Ross, R.G., Freedman, R., 2004. Varied effects of atypical neuroleptics on P50 auditory gating in schizophrenia patients. Am J Psychiatry. 161 (10), 1822-1828.

Adler, L.E., Olincy, A., Waldo, M., Harris, J.G., Griffith, J., Stevens, K., Flach, K., Nagamoto, H., Bickford, P., Leonard, S., Freedman, R., 1998. Schizophrenia, sensory gating, and nicotinic receptors. Schizophr Bull. 24 (2), 189-202.

Adler, L.E., Pachtman, E., Franks, R.D., Pecevich, M., Waldo, M.C., Freedman, R., 1982. Neurophysiological evidence for a defect in neuronal mechanisms involved in sensory gating in schizophrenia. Biol Psychiatry. 17 (6), 639-654.
Adler, L.E., Waldo, M.C., Freedman, R., 1985. Neurophysiologic studies of sensory gating in schizophrenia: comparison of auditory and visual responses. Biol Psychiatry. 20 (12), 1284-1296.

Adler, L.E., Waldo, M.C., Tatcher, A., Cawthra, E., Baker, N., Freedman, R., 1990b. Lack of relationship of auditory gating defects to negative symptoms in schizophrenia. Schizophr Res. 3 (2), 131138.

Aidelbaum, R., Labelle, A., Baddeley, A., Knott, V., 2018. Assessing the acute effects of CDP-choline on sensory gating in schizophrenia: A pilot study. J Psychopharmacol. 32 (5), 541-551.

AmericanPsychiatricAssociation, 2013. Diagnostic and statistical manual of mental disorders. American Psychiatric Association, Arlington, VA, USA.

Ancin, I., Cabranes, J.A., Vazquez-Alvarez, B., Santos, J.L., Sanchez-Morla, E., GarciaJimenez, M.A., Fernandez, C., Barabash, A., 2011. Sensory gating deficit is associated with catechol-O-methyltransferase polymorphisms in bipolar disorder. World $\mathbf{J}$ Biol Psychiatry. 12 (5), 376-384.

Arango, C., Summerfelt, A., Buchanan, R.W., 2003. Olanzapine effects on auditory sensory gating in schizophrenia. Am J Psychiatry. 160 (11), 2066-2068.

Arnfred, S.M., Chen, A.C., Glenthoj, B.Y., Hemmingsen, R.P., 2003. Normal p50 gating in unmedicated schizophrenia outpatients. Am J Psychiatry. 160 (12), 2236-2238.

Arnfred, S.M., Lind, N.M., Gjedde, A., Hansen, A.K., 2004. Scalp recordings of mid-latency AEP and auditory gating in the Gottingen 
minipig: a new animal model in information processing research. Int J Psychophysiol. 52 (3), 267-275.

Atagun, M.I., 2016. Brain oscillations in bipolar disorder and lithium-induced changes. Neuropsychiatr Dis Treat. 12, 589-601.

Atagun, M.I., Akin, B., Algin, O., Şıkoğlu, E.M., Can, S.S., Ulusoy Kaymak, S., Caykoylu, A., Moore, C.M., Phillips, M., Ongur, D., 2019. Intrinsic oscillations of auditory networks in schizophrenia and bipolar disorder. Dusunen Adam. Journal of Psychiatry and Neurological Sciences. 32 (4), 4-13.

Atagun, M.I., Guntekin, B., Masali, B., Tulay, E., Basar, E., 2014. Decrease of eventrelated delta oscillations in euthymic patients with bipolar disorder. Psychiatry Res. 223 (1), 43-48.

Atagun, M.I., Guntekin, B., Ozerdem, A., Tulay, E., Basar, E., 2013. Decrease of theta response in euthymic bipolar patients during an oddball paradigm. Cogn Neurodyn. 7 (3), 213-223.

Atagun, M.I., Guntekin, B., Tan, D., Tulay, E.E., Basar, E., 2015a. Lithium excessively enhances event related beta oscillations in patients with bipolar disorder. J Affect Disord. 170, 59-65.

Atagun, M.I., Sikoglu, E.M., Can, S.S., Karakas-Ugurlu, G., Ulusoy-Kaymak, S., Caykoylu, A., Algin, O., Phillips, M.L., Moore, C.M., Ongur, D., 2015 b. Investigation of Heschl's gyrus and planum temporale in patients with schizophrenia and bipolar disorder: a proton magnetic resonance spectroscopy study. Schizophr Res. 161 (2-3), 202-209.

Atagun, M.I., Sikoglu, E.M., Can, S.S., Ugurlu, G.K., Kaymak, S.U., Caykoylu, A., Algin, O., Phillips, M.L., Moore, C.M., Ongur, D., 2018. Neurochemical differences between bipolar disorder type I and II in superior temporal cortices: A proton magnetic resonance spectroscopy study. J Affect Disord. 235, 15-19.

Atagun, M.I., Sikoglu, E.M., Soykan, C., Serdar Suleyman, C., Ulusoy-Kaymak, S., Caykoylu, A., Algin, O., Phillips, M.L., Ongur, D., Moore, C.M., 2017. Perisylvian GABA levels in schizophrenia and bipolar disorder. Neurosci Lett. 637, 70-74.

Bak, N., Rostrup, E., Larsson, H.B., Glenthoj, B.Y., Oranje, B., 2014. Concurrent functional magnetic resonance imaging and electroencephalography assessment of sensory gating in schizophrenia. Hum Brain Mapp. 35 (8), 3578-3587.

Baker, N., Adler, L.E., Franks, R.D., Waldo, M., Berry, S., Nagamoto, H., Muckle, A., Freedman, R., 1987. Neurophysiological assessment of sensory gating in psychiatric inpatients: comparison between schizophrenia and other diagnoses. Biol Psychiatry. 22 (5), 603-617.

Başar, E., 1998. Brain oscillations I: principles and approaches. Springer, Heidelberg, Germany.

Başar, E., 1999. Brain function and oscillations: II. Integrative brain function. Neurophysiology and cognitive processes. Springer, Heidelberg, Germany.

Basar, E., Basar-Eroglu, C., Karakas, S., Schurmann, M., 1999. Are cognitive processes manifested in event-related gamma, alpha, theta and delta oscillations in the EEG? Neurosci Lett. 259 (3), 165-168.

Baslow, M.H., 2000. Functions of N-acetyl-Laspartate and $\mathrm{N}$-acetyl-L-aspartylglutamate in the vertebrate brain: role in glial cellspecific signaling. J Neurochem 75 (2), 453459. 
Bastiaansen, M., Hagoort, P., 2003. Eventinduced theta responses as a window on the dynamics of memory. Cortex. 39 (4-5), 967992.

Becker, J., Gomes, I., Ghisolfi, E.S., Schuch, A., Ramos, F.L., Ehlers, J.A., Nora, D.B., Lara, D.R., da Costa, J.C., 2004. Clozapine, but not typical antipsychotics, correct P50 suppression deficit in patients with schizophrenia. Clin Neurophysiol. 115 (2), 396-401.

Becker, J., Silva Filho, I.G., Filho, H.F., Schuch, A., Ramos, F.L., Ghisolfi, E.S., Lara, D.R., Costa, J.C., 2011. Pattern of P50 suppression deficit in patients with epilepsy and individuals with schizophrenia. Arq Neuropsiquiatr. 69 (3), 460-465.

Benes, F.M., Berretta, S., 2001. GABAergic interneurons: implications for understanding schizophrenia and bipolar disorder. Neuropsychopharmacology. 25 (1), 1-27.

Blumberg, H.P., Charney, D.S., Krystal, J.H., 2002. Frontotemporal neural systems in bipolar disorder. Semin Clin Neuropsychiatry. 7 (4), 243-254.

Bora, E., 2018. Neurocognitive features in clinical subgroups of bipolar disorder: A meta-analysis. J Affect Disord. 229, 125134.

Bora, E., Can, G., Ildiz, A., Ulas, G., Ongun, C.H., Inal, N.E., Ozerdem, A., 2019. Neurocognitive heterogeneity in young offspring of patients with bipolar disorder: The effect of putative clinical stages. $J$ Affect Disord. 257, 130-135.

Bortolato, B., Miskowiak, K.W., Kohler, C.A., Vieta, E., Carvalho, A.F., 2015. Cognitive dysfunction in bipolar disorder and schizophrenia: a systematic review of metaanalyses. Neuropsychiatr Dis Treat. 11, 3111-3125.
Boutros, N.N., Belger, A., Campbell, D., D'Souza, C., Krystal, J., 1999. Comparison of four components of sensory gating in schizophrenia and normal subjects: a preliminary report. Psychiatry Res. 88 (2), 119-130.

Boutros, N.N., Brockhaus-Dumke, A., Gjini, K., Vedeniapin, A., Elfakhani, M., Burroughs, S., Keshavan, M., 2009. Sensory-gating deficit of the N100 midlatency auditory evoked potential in medicated schizophrenia patients. Schizophr Res. 113 (2-3), 339-346.

Boutros, N.N., Korzyukov, O., Jansen, B., Feingold, A., Bell, M., 2004. Sensory gating deficits during the mid-latency phase of information processing in medicated schizophrenia patients. Psychiatry Res. 126 (3), 203-215.

Boutros, N.N., Zouridakis, G., Overall, J., 1991. Replication and extension of P50 findings in schizophrenia. Clin Electroencephalogr. 22 (1), 40-45.

Brady, R.O., Jr., McCarthy, J.M., Prescot, A.P., Jensen, J.E., Cooper, A.J., Cohen, B.M., Renshaw, P.F., Ongur, D., 2013. Brain gamma-aminobutyric acid (GABA) abnormalities in bipolar disorder. Bipolar Disord. 15 (4), 434-439.

Braff, D.L., Light, G.A., Swerdlow, N.R., 2007. Prepulse inhibition and P50 suppression are both deficient but not correlated in schizophrenia patients. Biol Psychiatry. 61 (10), 1204-1207.

Bramon, E., Rabe-Hesketh, S., Sham, P., Murray, R.M., Frangou, S., 2004. Metaanalysis of the P300 and P50 waveforms in schizophrenia. Schizophr Res. 70 (2-3), 315329.

Brenner, C.A., Kieffaber, P.D., Clementz, B.A., Johannesen, J.K., Shekhar, A., O'Donnell, B.F., Hetrick, W.P., 2009. Event-related 
potential abnormalities in schizophrenia: a failure to "gate in" salient information? Schizophr Res. 113 (2-3), 332-338.

Brockhaus-Dumke, A., Mueller, R., Faigle, U., Klosterkoetter, J., 2008a. Sensory gating revisited: relation between brain oscillations and auditory evoked potentials in schizophrenia. Schizophr Res. 99 (1-3), 238249.

Brockhaus-Dumke, A., Schultze-Lutter, F., Mueller, R., Tendolkar, I., Bechdolf, A., Pukrop, R., Klosterkoetter, J., Ruhrmann, S., 2008b. Sensory gating in schizophrenia: P50 and N100 gating in antipsychotic-free subjects at risk, first-episode, and chronic patients. Biol Psychiatry. 64 (5), 376-384.

Brunstein, M.G., Ghisolfi, E.S., Ramos, F.L., Lara, D.R., 2005. A clinical trial of adjuvant allopurinol therapy for moderately refractory schizophrenia. J Clin Psychiatry. 66 (2), 213-219.

Buchanan, R.W., Summerfelt, A., Tek, C., Gold, J., 2003. An open-labeled trial of adjunctive donepezil for cognitive impairments in patients with schizophrenia. Schizophr Res. 59 (1), 29-33.

Cabranes, J.A., Ancin, I., Santos, J.L., SanchezMorla, E., Garcia-Jimenez, M.A., LopezIbor, J.J., Barabash, A., 2013. No effect of polymorphisms in the non-duplicated region of the CHRNA7 gene on sensory gating P50 ratios in patients with schizophrenia and bipolar disorder. Psychiatry Res. 205 (3), 276-278.

Carroll, C.A., Kieffaber, P.D., Vohs, J.L., O'Donnell, B.F., Shekhar, A., Hetrick, W.P., 2008. Contributions of spectral frequency analyses to the study of P50 ERP amplitude and suppression in bipolar disorder with or without a history of psychosis. Bipolar Disord. 10 (7), 776-787.
Chang, W.P., Arfken, C.L., Sangal, M.P., Boutros, N.N., 2011. Probing the relative contribution of the first and second responses to sensory gating indices: a metaanalysis. Psychophysiology 48 (7), 980-992.

Chen, X.S., Li, C.B., Smith, R.C., Xiao, Z.P., Wang, J.J., 2011. Differential sensory gating functions between smokers and nonsmokers among drug-naive first episode schizophrenic patients. Psychiatry Res. 188 (3), 327-333.

Cheng, C.H., Chan, P.S., Liu, C.Y., Hsu, S.C., 2016. Auditory sensory gating in patients with bipolar disorders: A meta-analysis. J Affect Disord. 203, 199-203.

Chitty, K.M., Lagopoulos, J., Hickie, I.B., Hermens, D.F., 2015. Hippocampal glutamatergic/NMDA receptor functioning in bipolar disorder: A study combining mismatch negativity and proton magnetic resonance spectroscopy. Psychiatry Res. 233 (2), 88-94.

Chitty, K.M., Lagopoulos, J., Lee, R.S., Hickie, I.B., Hermens, D.F., 2013. A systematic review and meta-analysis of proton magnetic resonance spectroscopy and mismatch negativity in bipolar disorder. Eur Neuropsychopharmacol. 23 (11), 13481363.

Clementz, B.A., Blumenfeld, L.D., 2001. Multichannel electroencephalographic assessment of auditory evoked response suppression in schizophrenia. Exp Brain Res. 139 (4), 377-390.

Clementz, B.A., Blumenfeld, L.D., Cobb, S., 1997a. The gamma band response may account for poor P50 suppression in schizophrenia. Neuroreport 8 (18), 38893893.

Clementz, B.A., Geyer, M.A., Braff, D.L., 1997b. P50 suppression among schizophrenia and normal comparison 
subjects: a methodological analysis. Biol Psychiatry. 41 (10), 1035-1044.

Clementz, B.A., Geyer, M.A., Braff, D.L., 1998a. Multiple site evaluation of P50 suppression among schizophrenia and normal comparison subjects. Schizophr Res. 30 (1), 71-80.

Clementz, B.A., Geyer, M.A., Braff, D.L., 1998b. Poor P50 suppression among schizophrenia patients and their first-degree biological relatives. Am J Psychiatry. 155 (12), 1691-1694.

Cross-Disorder Group of the Psychiatric Genomics, C., 2013. Identification of risk loci with shared effects on five major psychiatric disorders: a genome-wide analysis. Lancet 381 (9875), 1371-1379.

Csomor, P.A., Preller, K.H., Geyer, M.A., Studerus, E., Huber, T., Vollenweider, F.X., 2014. Influence of aripiprazole, risperidone, and amisulpride on sensory and sensorimotor gating in healthy 'low and high gating' humans and relation to psychometry. Neuropsychopharmacology 39 (10), 24852496.

Csomor, P.A., Yee, B.K., Feldon, J., Theodoridou, A., Studerus, E., Vollenweider, F.X., 2009. Impaired prepulse inhibition and prepulse-elicited reactivity but intact reflex circuit excitability in unmedicated schizophrenia patients: a comparison with healthy subjects and medicated schizophrenia patients. Schizophr Bull. 35 (1), 244-255.

Cullum, C.M., Harris, J.G., Waldo, M.C., Smernoff, E., Madison, A., Nagamoto, H.T., Griffith, J., Adler, L.E., Freedman, R., 1993. Neurophysiological and neuropsychological evidence for attentional dysfunction in schizophrenia. Schizophr Res. 10 (2), 131141.
Cuthbert, B.N., 2014. The RDoC framework: facilitating transition from ICD/DSM to dimensional approaches that integrate neuroscience and psychopathology. World Psychiatry. 13 (1), 28-35.

Dalecki, A., Green, A.E., Johnstone, S.J., Croft, R.J., 2016. The relevance of attention in schizophrenia P50 paired stimulus studies. Clin Neurophysiol. 127 (6), 2448-2454.

de Almeida Rocca, C.C., de Macedo-Soares, M.B., Gorenstein, C., Tamada, R.S., Isller, C.K., Dias, R.S., de Almeida, K.M., Schwartzmann, A.M., Amaral, J.A., Lafer, B., 2008. Verbal fluency dysfunction in euthymic bipolar patients: a controlled study. J Affect Disord. 107 (1-3), 187-192.

de Wilde, O.M., Bour, L.J., Dingemans, P.M., Koelman, J.H., Linszen, D.H., 2007a. Failure to find P50 suppression deficits in young first-episode patients with schizophrenia and clinically unaffected siblings. Schizophr Bull. 33 (6), 1319-1323.

de Wilde, O.M., Bour, L.J., Dingemans, P.M., Koelman, J.H., Linszen, D.H., 2007b. A meta-analysis of P50 studies in patients with schizophrenia and relatives: differences in methodology between research groups. Schizophr Res. 97 (1-3), 137-151.

Demily, C., Louchart-de-la-Chapelle, S., Nkam, I., Ramoz, N., Denise, P., Nicolas, A., Savalle, C., Thibaut, F., 2016. Does COMT val158met polymorphism influence P50 sensory gating, eye tracking or saccadic inhibition dysfunctions in schizophrenia? Psychiatry Res. 246, 738-744.

Devrim-Ucok, M., Keskin-Ergen, H.Y., Ucok, A., 2008. P50 gating at acute and post-acute phases of first-episode schizophrenia. Prog Neuropsychopharmacol Biol Psychiatry. 32 (8), 1952-1956.

Dierks, T., Linden, D.E., Jandl, M., Formisano, E., Goebel, R., Lanfermann, H., Singer, W., 
1999. Activation of Heschl's gyrus during auditory hallucinations. Neuron 22 (3), 615621.

Doege, K., Jansen, M., Mallikarjun, P., Liddle, E.B., Liddle, P.F., 2010. How much does phase resetting contribute to event-related EEG abnormalities in schizophrenia? Neurosci Lett. 481 (1), 1-5.

Domjan, N., Csifcsak, G., Drotos, G., Janka, Z., Szendi, I., 2012. Different patterns of auditory information processing deficits in chronic schizophrenia and bipolar disorder with psychotic features. Schizophr Res. 139 (1-3), 253-259.

During, S., Glenthoj, B.Y., Andersen, G.S., Oranje, B., 2014. Effects of dopamine D2/D3 blockade on human sensory and sensorimotor gating in initially antipsychotic-naive, first-episode schizophrenia patients. Neuropsychopharmacology 39 (13), 30003008 .

Earls, H.A., Curran, T., Mittal, V., 2016. A Meta-analytic Review of Auditory EventRelated Potential Components as Endophenotypes for Schizophrenia: Perspectives From First-Degree Relatives. Schizophr Bull. 42 (6), 1504-1516.

Egerton, A., Modinos, G., Ferrera, D., McGuire, P., 2017. Neuroimaging studies of GABA in schizophrenia: a systematic review with meta-analysis. Transl Psychiatry. 7 (6), e1147.

El-Kaim, A., Aramaki, M., Ystad, S., KronlandMartinet, R., Cermolacce, M., Naudin, J., Vion-Dury, J., Micoulaud-Franchi, J.A., 2015. On the correlation between perceptual inundation caused by realistic immersive environmental auditory scenes and the sensory gating inventory in schizophrenia. Eur Psychiatry. 30 (5), 606-614.
Ergen, M., Marbach, S., Brand, A., BasarEroglu, C., Demiralp, T., 2008. P3 and delta band responses in visual oddball paradigm in schizophrenia. Neurosci Lett. 440 (3), 304308.

Erickson, M.A., Ruffle, A., Gold, J.M., 2016. A Meta-Analysis of Mismatch Negativity in Schizophrenia: From Clinical Risk to Disease Specificity and Progression. Biol Psychiatry. 79 (12), 980-987.

Ethridge, L.E., Hamm, J.P., Pearlson, G.D., Tamminga, C.A., Sweeney, J.A., Keshavan, M.S., Clementz, B.A., 2015. Event-related potential and time-frequency endophenotypes for schizophrenia and psychotic bipolar disorder. Biol Psychiatry. 77 (2), 127-136.

Frangou, S., Donaldson, S., Hadjulis, M., Landau, S., Goldstein, L.H., 2005. The Maudsley Bipolar Disorder Project: executive dysfunction in bipolar disorder I and its clinical correlates. Biol Psychiatry. 58 (11), 859-864.

Franks, R.D., Adler, L.E., Waldo, M.C., Alpert, J., Freedman, R., 1983. Neurophysiological studies of sensory gating in mania: comparison with schizophrenia. Biol Psychiatry. 18 (9), 989-1005.

Freedman, R., 2014. alpha7-nicotinic acetylcholine receptor agonists for cognitive enhancement in schizophrenia. Annu Rev Med. 65, 245-261.

Freedman, R., Adler, L.E., Gerhardt, G.A., Waldo, M., Baker, N., Rose, G.M., Drebing, C., Nagamoto, H., Bickford-Wimer, P., Franks, R., 1987. Neurobiological studies of sensory gating in schizophrenia. Schizophr Bull. 13 (4), 669-678.

Freedman, R., Adler, L.E., Waldo, M.C., Pachtman, E., Franks, R.D., 1983. Neurophysiological evidence for a defect in inhibitory pathways in schizophrenia: 
comparison of medicated and drug-free patients. Biol Psychiatry. 18 (5), 537-551.

Freedman, R., Coon, H., Myles-Worsley, M., Orr-Urtreger, A., Olincy, A., Davis, A., Polymeropoulos, M., Holik, J., Hopkins, J., Hoff, M., Rosenthal, J., Waldo, M.C., Reimherr, F., Wender, P., Yaw, J., Young, D.A., Breese, C.R., Adams, C., Patterson, D., Adler, L.E., Kruglyak, L., Leonard, S., Byerley, W., 1997. Linkage of a neurophysiological deficit in schizophrenia to a chromosome 15 locus. Proc Natl Acad Sci U S A. 94 (2), 587-592.

Fresan, A., Apiquian, R., Garcia-Anaya, M., de la Fuente-Sandoval, C., Nicolini, H., GraffGuerrero, A., 2007. The P50 auditory evoked potential in violent and non-violent patients with schizophrenia. Schizophr Res. 97 (1-3), 128-136.

Fridberg, D.J., Hetrick, W.P., Brenner, C.A., Shekhar, A., Steffen, A.N., Malloy, F.W., O'Donnell, B.F., 2009. Relationships between auditory event-related potentials and mood state, medication, and comorbid psychiatric illness in patients with bipolar disorder. Bipolar Disord. 11 (8), 857-866.

Gandal, M.J., Edgar, J.C., Klook, K., Siegel, S.J., 2012. Gamma synchrony: towards a translational biomarker for the treatmentresistant symptoms of schizophrenia. Neuropharmacology 62 (3), 1504-1518.

Garrido, M.I., Kilner, J.M., Stephan, K.E., Friston, K.J., 2009. The mismatch negativity: a review of underlying mechanisms. Clin Neurophysiol. 120 (3), 453-463.

Ghisolfi, E.S., Heldt, E., Zanardo, A.P., Strimitzer, I.M., Jr., Prokopiuk, A.S., Becker, J., Cordioli, A.V., Manfro, G.G., Lara, D.R., 2006. P50 sensory gating in panic disorder. J Psychiatr Res. 40 (6), 535540 .
Ghisolfi, E.S., Maegawa, G.H., Becker, J., Zanardo, A.P., Strimitzer, I.M., Jr., Prokopiuk, A.S., Pereira, M.L., Carvalho, T., Jardim, L.B., Lara, D.R., 2004. Impaired P50 sensory gating in Machado-Joseph disease. Clin Neurophysiol. 115 (10), 22312235 .

Ghisolfi, E.S., Prokopiuk, A.S., Becker, J., Ehlers, J.A., Belmonte-de-Abreu, P., Souza, D.O., Lara, D.R., 2002. The adenosine antagonist theophylline impairs p50 auditory sensory gating in normal subjects. Neuropsychopharmacology. 27 (4), 629637.

Gigante, A.D., Bond, D.J., Lafer, B., Lam, R.W., Young, L.T., Yatham, L.N., 2012. Brain glutamate levels measured by magnetic resonance spectroscopy in patients with bipolar disorder: a meta-analysis. Bipolar Disord. 14 (5), 478-487.

Gjini, K., Arfken, C., Boutros, N.N., 2010. Relationships between sensory "gating out" and sensory "gating in" of auditory evoked potentials in schizophrenia: a pilot study. Schizophr Res. 121 (1-3), 139-145.

Gjini, K., Burroughs, S., Boutros, N.N., 2011. Relevance of attention in auditory sensory gating paradigms in schizophrenia A pilot study. J Psychophysiol. 25 (2), 60-66.

Grande, I., Berk, M., Birmaher, B., Vieta, E., 2016. Bipolar disorder. Lancet 387 (10027), 1561-1572.

Greenwood, T.A., Light, G.A., Swerdlow, N.R., Calkins, M.E., Green, M.F., Gur, R.E., Gur, R.C., Lazzeroni, L.C., Nuechterlein, K.H., Olincy, A., Radant, A.D., Seidman, L.J., Siever, L.J., Silverman, J.M., Stone, W.S., Sugar, C.A., Tsuang, D.W., Tsuang, M.T., Turetsky, B.I., Freedman, R., Braff, D.L., 2016. Gating Deficit Heritability and Correlation With Increased Clinical Severity in Schizophrenia Patients With Positive 
Family History. Am J Psychiatry. 173 (4), 385-391.

Griffith, J.M., Freedman, R., 1995. Normalization of the auditory P50 gating deficit of schizophrenic patients after nonREM but not REM sleep. Psychiatry Res. 56 (3), 271-278.

Griffith, J.M., O'Neill, J.E., Petty, F., Garver, D., Young, D., Freedman, R., 1998. Nicotinic receptor desensitization and sensory gating deficits in schizophrenia. Biol Psychiatry. 44 (2), 98-106.

Griffith, J.M., Waldo, M., Adler, L.E., Freedman, R., 1993. Normalization of auditory sensory gating in schizophrenic patients after a brief period for sleep. Psychiatry Res. 49 (1), 29-39.

Guloksuz, S., van Os, J., 2018. The slow death of the concept of schizophrenia and the painful birth of the psychosis spectrum. Psychol Med 48 (2), 229-244.

Guntekin, B., Basar, E., 2016. Review of evoked and event-related delta responses in the human brain. Int J Psychophysiol 103, 43-52.

Hafeman, D.M., Chang, K.D., Garrett, A.S., Sanders, E.M., Phillips, M.L., 2012. Effects of medication on neuroimaging findings in bipolar disorder: an updated review. Bipolar Disord 14 (4), 375-410.

Hall, M.H., Chen, C.Y., Cohen, B.M., Spencer, K.M., Levy, D.L., Ongur, D., Smoller, J.W., 2015a. Genomewide association analyses of electrophysiological endophenotypes for schizophrenia and psychotic bipolar disorders: a preliminary report. Am J Med Genet B Neuropsychiatr Genet 168B (3), 151-161.

Hall, M.H., Jensen, J.E., Du, F., Smoller, J.W., O'Connor, L., Spencer, K.M., Ongur, D., 2015b. Frontal P3 event-related potential is related to brain glutamine/glutamate ratio measured in vivo. Neuroimage 111, 186191.

Hall, M.H., Levy, D.L., Salisbury, D.F., Haddad, S., Gallagher, P., Lohan, M., Cohen, B., Ongur, D., Smoller, J.W., 2014. Neurophysiologic effect of GWAS derived schizophrenia and bipolar risk variants. Am J Med Genet B Neuropsychiatr Genet 165B (1), 9-18.

Hall, M.H., Rijsdijk, F., Kalidindi, S., Schulze, K., Kravariti, E., Kane, F., Sham, P., Bramon, E., Murray, R.M., 2007a. Genetic overlap between bipolar illness and eventrelated potentials. Psychol Med 37 (5), 667678.

Hall, M.H., Rijsdijk, F., Picchioni, M., Schulze, K., Ettinger, U., Toulopoulou, T., Bramon, E., Murray, R.M., Sham, P., 2007 b. Substantial shared genetic influences on schizophrenia and event-related potentials. Am J Psychiatry 164 (5), 804-812.

Hall, M.H., Schulze, K., Rijsdijk, F., Picchioni, M., Ettinger, U., Bramon, E., Freedman, R., Murray, R.M., Sham, P., 2006. Heritability and reliability of P300, P50 and duration mismatch negativity. Behav Genet 36 (6), 845-857.

Hall, M.H., Schulze, K., Sham, P., Kalidindi, S., McDonald, C., Bramon, E., Levy, D.L., Murray, R.M., Rijsdijk, F., 2008. Further evidence for shared genetic effects between psychotic bipolar disorder and P50 suppression: a combined twin and family study. Am J Med Genet B Neuropsychiatr Genet 147B (5), 619-627.

Hamm, J.P., Ethridge, L.E., Shapiro, J.R., Pearlson, G.D., Tamminga, C.A., Sweeney, J.A., Keshavan, M.S., Thaker, G.K., Clementz, B.A., 2013. Family history of psychosis moderates early auditory cortical response abnormalities in non-psychotic 
bipolar disorder. Bipolar Disord 15 (7), 774786.

Hanford, L.C., Nazarov, A., Hall, G.B., Sassi, R.B., 2016. Cortical thickness in bipolar disorder: a systematic review. Bipolar Disord 18 (1), 4-18.

Harmony, T., 2013. The functional significance of delta oscillations in cognitive processing. Front Integr Neurosci 7, 83.

Hawkins, K.A., Keefe, R.S., Christensen, B.K., Addington, J., Woods, S.W., Callahan, J., Zipursky, R.B., Perkins, D.O., Tohen, M., Breier, A., McGlashan, T.H., 2008. Neuropsychological course in the prodrome and first episode of psychosis: findings from the PRIME North America Double Blind Treatment Study. Schizophr Res 105 (1-3), $1-9$.

Herold, F., Wiegel, P., Scholkmann, F., Muller, N.G., 2018. Applications of Functional Near-Infrared Spectroscopy (fNIRS) Neuroimaging in Exercise(-)Cognition Science: A Systematic, MethodologyFocused Review. J Clin Med 7 (12).

Hill, S.K., Ragland, J.D., Gur, R.C., Gur, R.E., 2001. Neuropsychological differences among empirically derived clinical subtypes of schizophrenia. Neuropsychology 15 (4), 492-501.

Hirayasu, Y., Shenton, M.E., Salisbury, D.F., Dickey, C.C., Fischer, I.A., Mazzoni, P., Kisler, T., Arakaki, H., Kwon, J.S., Anderson, J.E., Yurgelun-Todd, D., Tohen, M., McCarley, R.W., 1998. Lower left temporal lobe MRI volumes in patients with first-episode schizophrenia compared with psychotic patients with first-episode affective disorder and normal subjects. Am J Psychiatry 155 (10), 1384-1391.

Hoff, A.L., Wieneke, M., Faustman, W.O., Horon, R., Sakuma, M., Blankfeld, H., Espinoza, S., DeLisi, L.E., 1998. Sex differences in neuropsychological functioning of first-episode and chronically ill schizophrenic patients. Am J Psychiatry 155 (10), 1437-1439.

Holstein, D.H., Csomor, P.A., Geyer, M.A., Huber, T., Brugger, N., Studerus, E., Vollenweider, F.X., 2011. The effects of sertindole on sensory gating, sensorimotor gating, and cognition in healthy volunteers. J Psychopharmacol 25 (12), 1600-1613.

Hong, L.E., Buchanan, R.W., Thaker, G.K., Shepard, P.D., Summerfelt, A., 2008a. Beta $(\sim 16 \mathrm{~Hz})$ frequency neural oscillations mediate auditory sensory gating in humans. Psychophysiology 45 (2), 197-204.

Hong, L.E., Summerfelt, A., McMahon, R.P., Thaker, G.K., Buchanan, R.W., 2004. Gamma/beta oscillation and sensory gating deficit in schizophrenia. Neuroreport 15 (1), 155-159.

Hong, L.E., Summerfelt, A., Mitchell, B.D., McMahon, R.P., Wonodi, I., Buchanan, R.W., Thaker, G.K., 2008b. Sensory gating endophenotype based on its neural oscillatory pattern and heritability estimate. Arch Gen Psychiatry 65 (9), 1008-1016.

Hong, L.E., Summerfelt, A., Wonodi, I., Adami, H., Buchanan, R.W., Thaker, G.K., 2007. Independent domains of inhibitory gating in schizophrenia and the effect of stimulus interval. Am J Psychiatry 164 (1), 61-65.

Hong, X., Chan, R.C., Zhuang, X., Jiang, T., Wan, X., Wang, J., Xiao, B., Zhou, H., Jiang, L., Weng, B., 2009. Neuroleptic effects on P50 sensory gating in patients with firstepisode never-medicated schizophrenia. Schizophr Res 108 (1-3), 151-157.

Hsieh, M.H., Liu, K., Liu, S.K., Chiu, M.J., Hwu, H.G., Chen, A.C., 2004. Memory impairment and auditory evoked potential 
gating deficit in schizophrenia. Psychiatry Res 130 (2), 161-169.

Hsieh, M.H., Shan, J.C., Huang, W.L., Cheng, W.C., Chiu, M.J., Jaw, F.S., Hwu, H.G., Liu, C.C., 2012. Auditory event-related potential of subjects with suspected pre-psychotic state and first-episode psychosis. Schizophr Res 140 (1-3), 243-249.

Humpston, C.S., Adams, R.A., Benrimoh, D., Broome, M.R., Corlett, P.R., Gerrans, P., Horga, G., Parr, T., Pienkos, E., Powers, A.R., 3rd, Raballo, A., Rosen, C., Linden, D.E.J., 2019. From Computation to the FirstPerson: Auditory-Verbal Hallucinations and Delusions of Thought Interference in Schizophrenia-Spectrum Psychoses. Schizophr Bull 45 (45 Suppl 1), S56-S66.

Irani, F., Kalkstein, S., Moberg, E.A., Moberg, P.J., 2011. Neuropsychological performance in older patients with schizophrenia: a metaanalysis of cross-sectional and longitudinal studies. Schizophr Bull 37 (6), 1318-1326.

Javitt, D.C., 2009. When doors of perception close: bottom-up models of disrupted cognition in schizophrenia. Annu Rev Clin Psychol 5, 249-275.

Jin, Y., Bunney, W.E., Jr., Sandman, C.A., Patterson, J.V., Fleming, K., Moenter, J.R., Kalali, A.H., Hetrick, W.P., Potkin, S.G., 1998. Is P50 suppression a measure of sensory gating in schizophrenia? Biol Psychiatry 43 (12), 873-878.

Johannesen, J.K., Kieffaber, P.D., O'Donnell, B.F., Shekhar, A., Evans, J.D., Hetrick, W.P., 2005. Contributions of subtype and spectral frequency analyses to the study of P50 ERP amplitude and suppression in schizophrenia. Schizophr Res 78 (2-3), 269284.

Johannesen, J.K., O'Donnell, B.F., Shekhar, A., McGrew, J.H., Hetrick, W.P., 2013. Diagnostic specificity of neurophysiological endophenotypes in schizophrenia and bipolar disorder. Schizophr Bull 39 (6), 1219-1229.

Jones, L.A., Hills, P.J., Dick, K.M., Jones, S.P., Bright, P., 2016. Cognitive mechanisms associated with auditory sensory gating. Brain Cogn 102, 33-45.

Judd, L.L., McAdams, L., Budnick, B., Braff, D.L., 1992. Sensory gating deficits in schizophrenia: new results. Am J Psychiatry 149 (4), 488-493.

Kathmann, N., Engel, R.R., 1990. Sensory gating in normals and schizophrenics: a failure to find strong P50 suppression in normals. Biol Psychiatry 27 (11), 12161226.

Kato, T., 2019. Current understanding of bipolar disorder: Toward integration of biological basis and treatment strategies. Psychiatry Clin Neurosci 73 (9), 526-540.

Kaur, M., Chitty, K.M., Lagopoulos, J., Hickie, I.B., Duffy, S.L., Hermens, D.F., 2019. Elucidating the glutamatergic processes underlying mismatch negativity deficits in early stage bipolar disorder and schizophrenia: A combined (1)H-MRS and EEG study. J Psychiatr Res 113, 83-89.

Kaya, E., Aydemir, O., Selcuki, D., 2007. Residual symptoms in bipolar disorder: the effect of the last episode after remission. Prog Neuropsychopharmacol Biol Psychiatry 31 (7), 1387-1392.

Kelly, S., Guimond, S., Lyall, A., Stone, W.S., Shenton, M.E., Keshavan, M., Seidman, L.J., 2018. Neural correlates of cognitive deficits across developmental phases of schizophrenia. Neurobiol Dis.

Kenemans, J.L., Kahkonen, S., 2011. How human electrophysiology informs psychopharmacology: from bottom-up 
driven processing to top-down control. Neuropsychopharmacology 36 (1), 26-51.

Keri, S., Beniczky, S., Kelemen, O., 2010. Suppression of the P50 evoked response and neuregulin 1-induced AKT phosphorylation in first-episode schizophrenia. Am J Psychiatry 167 (4), 444-450.

Keshavan, M.S., Ongur, D., 2014. The journey from RDC/DSM diagnoses toward RDoC dimensions. World Psychiatry 13 (1), 44-46.

Kim, M., Yoon, Y.B., Lee, T.H., Lee, T.Y., Kwon, J.S., 2018. The effect of tDCS on auditory hallucination and P50 sensory gating in patients with schizophrenia: A pilot study. Schizophr Res 192, 469-470.

Kim, Y., Kwon, A., Min, D., Kim, S., Jin, M.J., Lee, S.H., 2019. Neurophysiological and Psychological Predictors of Social Functioning in Patients with Schizophrenia and Bipolar Disorder. Psychiatry Investig 16 (10), 718-727.

Kisley, M.A., Cornwell, Z.M., 2006. Gamma and beta neural activity evoked during a sensory gating paradigm: effects of auditory, somatosensory and cross-modal stimulation. Clin Neurophysiol 117 (11), 2549-2563.

Klimesch, W., 1999. EEG alpha and theta oscillations reflect cognitive and memory performance: a review and analysis. Brain Res Brain Res Rev 29 (2-3), 169-195.

Knyazev, G.G., 2012. EEG delta oscillations as a correlate of basic homeostatic and motivational processes. Neurosci Biobehav Rev 36 (1), 677-695.

Koike, K., Hashimoto, K., Takai, N., Shimizu, E., Komatsu, N., Watanabe, H., Nakazato, M., Okamura, N., Stevens, K.E., Freedman, R., Iyo, M., 2005. Tropisetron improves deficits in auditory P50 suppression in schizophrenia. Schizophr Res 76 (1), 67-72.
Kurtz, M.M., Gerraty, R.T., 2009. A metaanalytic investigation of neurocognitive deficits in bipolar illness: profile and effects of clinical state. Neuropsychology 23 (5), 551-562.

Lee, P.S., Chen, Y.S., Hsieh, J.C., Su, T.P., Chen, L.F., 2010. Distinct neuronal oscillatory responses between patients with bipolar and unipolar disorders: a magnetoencephalographic study. J Affect Disord 123 (1-3), 270-275.

Lenox, R.H., Hahn, C.G., 2000. Overview of the mechanism of action of lithium in the brain: fifty-year update. J Clin Psychiatry 61 Suppl 9, 5-15.

Lewandowski, K.E., Cohen, B.M., Ongur, D., 2011. Evolution of neuropsychological dysfunction during the course of schizophrenia and bipolar disorder. Psychol Med 41 (2), 225-241.

Lieberman, J.A., First, M.B., 2018. Psychotic Disorders. N Engl J Med 379 (3), 270-280.

Light, G.A., Geyer, M.A., Clementz, B.A., Cadenhead, K.S., Braff, D.L., 2000. Normal P50 suppression in schizophrenia patients treated with atypical antipsychotic medications. Am J Psychiatry 157 (5), 767 771.

Lijffijt, M., Moeller, F.G., Boutros, N.N., Steinberg, J.L., Meier, S.L., Lane, S.D., Swann, A.C., 2009. Diminished P50, N100 and P200 auditory sensory gating in bipolar I disorder. Psychiatry Res 167 (3), 191-201.

Linden, D.E., Thornton, K., Kuswanto, C.N., Johnston, S.J., van de Ven, V., Jackson, M.C., 2011. The brain's voices: comparing nonclinical auditory hallucinations and imagery. Cereb Cortex 21 (2), 330-337.

Louchart-de la Chapelle, S., Levillain, D., Menard, J.F., Van der Elst, A., Allio, G., Haouzir, S., Dollfus, S., Campion, D., 
Thibaut, F., 2005a. P50 inhibitory gating deficit is correlated with the negative symptomatology of schizophrenia. Psychiatry Res 136 (1), 27-34.

Louchart-de la Chapelle, S., Nkam, I., Houy, E., Belmont, A., Menard, J.F., Roussignol, A.C., Siwek, O., Mezerai, M., Guillermou, M., Fouldrin, G., Levillain, D., Dollfus, S., Campion, D., Thibaut, F., 2005b. A concordance study of three electrophysiological measures in schizophrenia. Am J Psychiatry 162 (3), 466-474.

Lu, B.Y., Martin, K.E., Edgar, J.C., Smith, A.K., Lewis, S.F., Escamilla, M.A., Miller, G.A., Canive, J.M., 2007. Effect of catechol O-methyltransferase $\quad \operatorname{val}(158) \mathrm{met}$ polymorphism on the p50 gating endophenotype in schizophrenia. Biol Psychiatry 62 (7), 822-825.

Lundin, N.B., Bartolomeo, L.A., O'Donnell, B.F., Hetrick, W.P., 2018. Reduced electroencephalogram responses to standard and target auditory stimuli in bipolar disorder and the impact of psychotic features: Analysis of event-related potentials, spectral power, and inter-trial coherence. Bipolar Disord 20 (1), 49-59.

MacCabe, J.H., Wicks, S., Lofving, S., David, A.S., Berndtsson, A., Gustafsson, J.E., Allebeck, P., Dalman, C., 2013. Decline in cognitive performance between ages 13 and 18 years and the risk for psychosis in adulthood: a Swedish longitudinal cohort study in males. JAMA Psychiatry 70 (3), 261-270.

Maddock, R.J., Buonocore, M.H., 2012. MR spectroscopic studies of the brain in psychiatric disorders. Curr Top Behav Neurosci 11, 199-251.

Malhi, G.S., Ivanovski, B., Hadzi-Pavlovic, D., Mitchell, P.B., Vieta, E., Sachdev, P., 2007. Neuropsychological deficits and functional impairment in bipolar depression, hypomania and euthymia. Bipolar Disord 9 (1-2), 114-125.

Mann, C., Croft, R.J., Scholes, K.E., Dunne, A., O'Neill, B.V., Leung, S., Copolov, D., Phan, K.L., Nathan, P.J., 2008. Differential effects of acute serotonin and dopamine depletion on prepulse inhibition and p50 suppression measures of sensorimotor and sensory gating in humans. Neuropsychopharmacology 33 (7), 16531666.

Martin, L.F., Hall, M.H., Ross, R.G., Zerbe, G., Freedman, R., Olincy, A., 2007a. Physiology of schizophrenia, bipolar disorder, and schizoaffective disorder. Am J Psychiatry 164 (12), 1900-1906.

Martin, L.F., Leonard, S., Hall, M.H., Tregellas, J.R., Freedman, R., Olincy, A., 2007b. Sensory gating and alpha-7 nicotinic receptor gene allelic variants in schizoaffective disorder, bipolar type. Am J Med Genet B Neuropsychiatr Genet 144B (5), 611-614.

Martinez-Aran, A., Vieta, E., Reinares, M., Colom, F., Torrent, C., Sanchez-Moreno, J., Benabarre, A., Goikolea, J.M., Comes, M., Salamero, M., 2004. Cognitive function across manic or hypomanic, depressed, and euthymic states in bipolar disorder. Am J Psychiatry 161 (2), 262-270.

Mazhari, S., Price, G., Waters, F., Dragovic, M., Jablensky, A., 2011. Evidence of abnormalities in mid-latency auditory evoked responses (MLAER) in cognitive subtypes of patients with schizophrenia. Psychiatry Res 187 (3), 317-323.

McCormick, D.A., Pape, H.C., 1990. Properties of a hyperpolarization-activated cation current and its role in rhythmic oscillation in thalamic relay neurones. J Physiol 431, 291318. 
McNamara, R.K., Rider, T., Jandacek, R., Tso, P., 2014. Abnormal fatty acid pattern in the superior temporal gyrus distinguishes bipolar disorder from major depression and schizophrenia and resembles multiple sclerosis. Psychiatry Res 215 (3), 560-567.

Meffre, D., Massaad, C., Grenier, J., 2015. Lithium chloride stimulates PLP and MBP expression in oligodendrocytes via Wnt/beta-catenin and Akt/CREB pathways. Neuroscience 284, 962-971.

Merikangas, K.R., Akiskal, H.S., Angst, J., Greenberg, P.E., Hirschfeld, R.M., Petukhova, M., Kessler, R.C., 2007. Lifetime and 12-month prevalence of bipolar spectrum disorder in the National Comorbidity Survey replication. Arch Gen Psychiatry 64 (5), 543-552.

Merritt, K., Egerton, A., Kempton, M.J., Taylor, M.J., McGuire, P.K., 2016. Nature of Glutamate Alterations in Schizophrenia: A Meta-analysis of Proton Magnetic Resonance Spectroscopy Studies. JAMA Psychiatry 73 (7), 665-674.

Micoulaud-Franchi, J.A., Aramaki, M., Geoffroy, P.A., Richieri, R., Cermolacce, M., Faget, C., Ystad, S., Kronland-Martinet, R., Lancon, C., Vion-Dury, J., 2015a. Effects of clozapine on perceptual abnormalities and sensory gating: a preliminary cross-sectional study in schizophrenia. J Clin Psychopharmacol 35 (2), 184-187.

Micoulaud-Franchi, J.A., Aramaki, M., Merer, A., Cermolacce, M., Ystad, S., KronlandMartinet, R., Naudin, J., Vion-Dury, J., 2012. Toward an exploration of feeling of strangeness in schizophrenia: perspectives on acousmatic and everyday listening. $\mathrm{J}$ Abnorm Psychol 121 (3), 628-640.

Micoulaud-Franchi, J.A., Faugere, M., Boyer, L., Cermolacce, M., Richieri, R., Faget, C., Philip, P., Vion-Dury, J., Lancon, C., 2015 b.
Association of metabolic syndrome with sensory gating deficits in patients with chronic schizophrenia. Psychoneuroendocrinology 57, 125-133.

Micoulaud-Franchi, J.A., Hetrick, W.P., Aramaki, M., Bolbecker, A., Boyer, L., Ystad, S., Kronland-Martinet, R., Richieri, R., Faget, C., Faugere, M., El-Kaim, A., Cermolacce, M., Lancon, C., Vion-Dury, J., 2014. Do schizophrenia patients with low P50-suppression report more perceptual anomalies with the sensory gating inventory? Schizophr Res 157 (1-3), 157162.

Micoulaud-Franchi, J.A., Vaillant, F., Lopez, R., Peri, P., Baillif, A., Brandejsky, L., Steffen, M.L., Boyer, L., Richieri, R., Cermolacce, M., Bioulac, S., Aramaki, M., Philip, P., Lancon, C., Vion-Dury, J., 2015c. Sensory gating in adult with attentiondeficit/hyperactivity disorder: Event-evoked potential and perceptual experience reports comparisons with schizophrenia. Biol Psychol 107, 16-23.

Milev, P., Ho, B.C., Arndt, S., Andreasen, N.C., 2005. Predictive values of neurocognition and negative symptoms on functional outcome in schizophrenia: a longitudinal first-episode study with 7-year follow-up. Am J Psychiatry 162 (3), 495-506.

Modinos, G., Costafreda, S.G., van Tol, M.J., McGuire, P.K., Aleman, A., Allen, P., 2013. Neuroanatomy of auditory verbal hallucinations in schizophrenia: a quantitative meta-analysis of voxel-based morphometry studies. Cortex 49 (4), 10461055.

Moffett, J.R., Ross, B., Arun, P., Madhavarao, C.N., Namboodiri, A.M., 2007. NAcetylaspartate in the CNS: from neurodiagnostics to neurobiology. Prog Neurobiol 81 (2), 89-131. 
Moher, D., Liberati, A., Tetzlaff, J., Altman, D.G., Group, P., 2009. Preferred reporting items for systematic reviews and metaanalyses: the PRISMA statement. PLoS Med 6 (7), e1000097.

Mokhtari, M., Narayanan, B., Hamm, J.P., Soh, P., Calhoun, V.D., Ruano, G., Kocherla, M., Windemuth, A., Clementz, B.A., Tamminga, C.A., Sweeney, J.A., Keshavan, M.S., Pearlson, G.D., 2016. Multivariate Genetic Correlates of the Auditory Paired Stimuli-Based P2 Event-Related Potential in the Psychosis Dimension From the BSNIP Study. Schizophr Bull 42 (3), 851-862.

Moore, D.R., 2002. Auditory development and the role of experience. Br Med Bull 63, 171181.

Moore, G.J., Galloway, M.P., 2002. Magnetic resonance spectroscopy: neurochemistry and treatment effects in affective disorders. Psychopharmacol Bull 36 (2), 5-23.

Moran, Z.D., Williams, T.J., Bachman, P., Nuechterlein, K.H., Subotnik, K.L., Yee, C.M., 2012. Spectral decomposition of P50 suppression in schizophrenia during concurrent visual processing. Schizophr Res 140 (1-3), 237-242.

Mullins, P.G., McGonigle, D.J., O'Gorman, R.L., Puts, N.A., Vidyasagar, R., Evans, C.J., Cardiff Symposium on, M.R.S.o.G., Edden, R.A., 2014. Current practice in the use of MEGA-PRESS spectroscopy for the detection of GABA. Neuroimage 86, 43-52.

Myles-Worsley, M., 2002. P50 sensory gating in multiplex schizophrenia families from a Pacific island isolate. Am J Psychiatry 159 (12), 2007-2012.

Myles-Worsley, M., Ord, L., Blailes, F., Ngiralmau, H., Freedman, R., 2004. P50 sensory gating in adolescents from a pacific island isolate with elevated risk for schizophrenia. Biol Psychiatry 55 (7), 663667.

Nacher, V., Ledberg, A., Deco, G., Romo, R., 2013. Coherent delta-band oscillations between cortical areas correlate with decision making. Proc Natl Acad Sci U S A 110 (37), 15085-15090.

Nagamoto, H.T., Adler, L.E., Hea, R.A., Griffith, J.M., McRae, K.A., Freedman, R., 1996. Gating of auditory P50 in schizophrenics: unique effects of clozapine. Biol Psychiatry 40 (3), 181-188.

Nagamoto, H.T., Adler, L.E., McRae, K.A., Huettl, P., Cawthra, E., Gerhardt, G., Hea, R., Griffith, J., 1999. Auditory P50 in schizophrenics on clozapine: improved gating parallels clinical improvement and changes in plasma 3-methoxy-4hydroxyphenylglycol. Neuropsychobiology 39 (1), 10-17.

Nagamoto, H.T., Adler, L.E., Waldo, M.C., Freedman, R., 1989. Sensory gating in schizophrenics and normal controls: effects of changing stimulation interval. Biol Psychiatry 25 (5), 549-561.

Nagamoto, H.T., Adler, L.E., Waldo, M.C., Griffith, J., Freedman, R., 1991. Gating of auditory response in schizophrenics and normal controls. Effects of recording site and stimulation interval on the P50 wave. Schizophr Res 4 (1), 31-40.

Narayanan, B., Ethridge, L.E., O'Neil, K., Dunn, S., Mathew, I., Tandon, N., Calhoun, V.D., Ruano, G., Kocherla, M., Windemuth, A., Clementz, B.A., Tamminga, C.A., Sweeney, J.A., Keshavan, M.S., Pearlson, G.D., 2015. Genetic Sources of Subcomponents of Event-Related Potential in the Dimension of Psychosis Analyzed From the B-SNIP Study. Am J Psychiatry $172(5), 466-478$. 
Narayanan, B., O'Neil, K., Berwise, C., Stevens, M.C., Calhoun, V.D., Clementz, B.A., Tamminga, C.A., Sweeney, J.A., Keshavan, M.S., Pearlson, G.D., 2014. Resting state electroencephalogram oscillatory abnormalities in schizophrenia and psychotic bipolar patients and their relatives from the bipolar and schizophrenia network on intermediate phenotypes study. Biol Psychiatry 76 (6), 456-465.

Neuhaus, A.H., Popescu, F.C., Rentzsch, J., Gallinat, J., 2014. Critical evaluation of auditory event-related potential deficits in schizophrenia: evidence from large-scale single-subject pattern classification. Schizophr Bull 40 (5), 1062-1071.

Niznikiewicz, M.A., 2019. Neurobiological approaches to the study of clinical and genetic high risk for developing psychosis. Psychiatry Res 277, 17-22.

Nudmamud, S., Reynolds, L.M., Reynolds, G.P., 2003. N-acetylaspartate and NAcetylaspartylglutamate deficits in superior temporal cortex in schizophrenia and bipolar disorder: a postmortem study. Biol Psychiatry 53 (12), 1138-1141.

O'Donnell, B.F., Faux, S.F., McCarley, R.W., Kimble, M.O., Salisbury, D.F., Nestor, P.G., Kikinis, R., Jolesz, F.A., Shenton, M.E., 1995. Increased rate of P300 latency prolongation with age in schizophrenia. Electrophysiological evidence for a neurodegenerative process. Arch Gen Psychiatry 52 (7), 544-549.

O'Donnell, B.F., Hetrick, W.P., Vohs, J.L., Krishnan, G.P., Carroll, C.A., Shekhar, A., 2004a. Neural synchronization deficits to auditory stimulation in bipolar disorder. Neuroreport 15 (8), 1369-1372.

O'Donnell, B.F., Vohs, J.L., Hetrick, W.P., Carroll, C.A., Shekhar, A., 2004b. Auditory event-related potential abnormalities in bipolar disorder and schizophrenia. Int $\mathbf{J}$ Psychophysiol 53 (1), 45-55.

O'Neill, H.C., Schmitt, M.P., Stevens, K.E., 2003. Lithium alters measures of auditory gating in two strains of mice. Biol Psychiatry 54 (8), 847-853.

Oda, Y., Onitsuka, T., Tsuchimoto, R., Hirano, S., Oribe, N., Ueno, T., Hirano, Y., Nakamura, I., Miura, T., Kanba, S., 2012. Gamma band neural synchronization deficits for auditory steady state responses in bipolar disorder patients. PLoS One 7 (7), e39955.

Olincy, A., Braff, D.L., Adler, L.E., Cadenhead, K.S., Calkins, M.E., Dobie, D.J., Green, M.F., Greenwood, T.A., Gur, R.E., Gur, R.C., Light, G.A., Mintz, J., Nuechterlein, K.H., Radant, A.D., Schork, N.J., Seidman, L.J., Siever, L.J., Silverman, J.M., Stone, W.S., Swerdlow, N.R., Tsuang, D.W., Tsuang, M.T., Turetsky, B.I., Wagner, B.D., Freedman, R., 2010. Inhibition of the P50 cerebral evoked response to repeated auditory stimuli: results from the Consortium on Genetics of Schizophrenia. Schizophr Res 119 (1-3), 175-182.

Olincy, A., Martin, L., 2005. Diminished suppression of the P50 auditory evoked potential in bipolar disorder subjects with a history of psychosis. Am J Psychiatry 162 (1), 43-49.

Olincy, A., Ross, R.G., Harris, J.G., Young, D.A., McAndrews, M.A., Cawthra, E., McRae, K.A., Sullivan, B., Adler, L.E., Freedman, R., 2000. The P50 auditory event-evoked potential in adult attentiondeficit disorder: comparison with schizophrenia. Biol Psychiatry 47 (11), 969977.

Ongur, D., Prescot, A.P., Jensen, J.E., Cohen, B.M., Renshaw, P.F., 2009. Creatine abnormalities in schizophrenia and bipolar disorder. Psychiatry Res 172 (1), 44-48. 
Oranje, B., Aggernaes, B., Rasmussen, H., Ebdrup, B.H., Glenthoj, B.Y., 2013. P50 suppression and its neural generators in antipsychotic-naive first-episode schizophrenia before and after 6 months of quetiapine treatment. Schizophr Bull 39 (2), 472-480.

Oranje, B., Glenthoj, B.Y., 2014. Clonidine normalizes levels of P50 gating in patients with schizophrenia on stable medication. Schizophr Bull 40 (5), 1022-1029.

Oranje, B., Van Oel, C.J., Gispen-De Wied, C.C., Verbaten, M.N., Kahn, R.S., 2002. Effects of typical and atypical antipsychotics on the prepulse inhibition of the startle reflex in patients with schizophrenia. J Clin Psychopharmacol 22 (4), 359-365.

Oranje, B., Wienberg, M., Glenthoj, B.Y., 2011. A single high dose of escitalopram disrupts sensory gating and habituation, but not sensorimotor gating in healthy volunteers. Psychiatry Res 186 (2-3), 431-436.

Oribe, N., Hirano, Y., Kanba, S., Del Re, E., Seidman, L., Mesholam-Gately, R., Goldstein, J.M., Shenton, M., Spencer, K.M., McCarley, R.W., Niznikiewicz, M., 2015. Progressive reduction of visual P300 amplitude in patients with first-episode schizophrenia: an ERP study. Schizophr Bull 41 (2), 460-470.

Oribe, N., Onitsuka, T., Hirano, S., Hirano, Y., Maekawa, T., Obayashi, C., Ueno, T., Kasai, K., Kanba, S., 2010. Differentiation between bipolar disorder and schizophrenia revealed by neural oscillation to speech sounds: an MEG study. Bipolar Disord 12 (8), 804-812.

Painold, A., Faber, P.L., Milz, P., Reininghaus, E.Z., Holl, A.K., Letmaier, M., PascualMarqui, R.D., Reininghaus, B., Kapfhammer, H.P., Lehmann, D., 2014. Brain electrical source imaging in manic and depressive episodes of bipolar disorder. Bipolar Disord 16 (7), 690-702.
Parker, D.A., Hamm, J.P., McDowell, J.E., Keedy, S.K., Gershon, E.S., Ivleva, E.I., Pearlson, G.D., Keshavan, M.S., Tamminga, C.A., Sweeney, J.A., Clementz, B.A., 2019. Auditory steady-state EEG response across the schizo-bipolar spectrum. Schizophr Res 209, 218-226.

Patterson, J.V., Hetrick, W.P., Boutros, N.N., Jin, Y., Sandman, C., Stern, H., Potkin, S., Bunney, W.E., Jr., 2008. P50 sensory gating ratios in schizophrenics and controls: a review and data analysis. Psychiatry Res 158 (2), 226-247.

Patterson, J.V., Sandman, C.A., Ring, A., Jin, Y., Bunney, W.E., Jr., 2009. An initial report of a new biological marker for bipolar disorder: P85 evoked brain potential. Bipolar Disord 11 (6), 596-609.

Pavuluri, M.N., West, A., Hill, S.K., Jindal, K., Sweeney, J.A., 2009. Neurocognitive function in pediatric bipolar disorder: 3-year follow-up shows cognitive development lagging behind healthy youths. J Am Acad Child Adolesc Psychiatry 48 (3), 299-307.

Petersen, S.E., Sporns, O., 2015. Brain Networks and Cognitive Architectures. Neuron 88 (1), 207-219.

Phillips, M.L., Drevets, W.C., Rauch, S.L., Lane, R., 2003. Neurobiology of emotion perception I: The neural basis of normal emotion perception. Biol Psychiatry 54 (5), 504-514.

Poels, E.M., Kegeles, L.S., Kantrowitz, J.T., Javitt, D.C., Lieberman, J.A., Abi-Dargham, A., Girgis, R.R., 2014. Glutamatergic abnormalities in schizophrenia: a review of proton MRS findings. Schizophr Res 152 (23), 325-332.

Ponton, C., Eggermont, J.J., Khosla, D., Kwong, B., Don, M., 2002. Maturation of human central auditory system activity: separating auditory evoked potentials by 
dipole source modeling. Clin Neurophysiol 113 (3), 407-420.

Potter, D., Summerfelt, A., Gold, J., Buchanan, R.W., 2006. Review of clinical correlates of P50 sensory gating abnormalities in patients with schizophrenia. Schizophr Bull 32 (4), 692-700.

Price, G.W., Michie, P.T., Johnston, J., InnesBrown, H., Kent, A., Clissa, P., Jablensky, A.V., 2006. A multivariate electrophysiological endophenotype, from a unitary cohort, shows greater research utility than any single feature in the Western Australian family study of schizophrenia. Biol Psychiatry 60 (1), 1-10.

Rass, O., Krishnan, G., Brenner, C.A., Hetrick, W.P., Merrill, C.C., Shekhar, A., O'Donnell, B.F., 2010. Auditory steady state response in bipolar disorder: relation to clinical state, cognitive performance, medication status, and substance disorders. Bipolar Disord 12 (8), 793-803.

Rauschecker, J.P., Scott, S.K., 2009. Maps and streams in the auditory cortex: nonhuman primates illuminate human speech processing. Nat Neurosci 12 (6), 718-724.

Reinvang, I., 1999. Cognitive event-related potentials in neuropsychological assessment. Neuropsychol Rev 9 (4), 231248.

Ren, Y., Wang, H., Xiao, L., 2013. Improving myelin/oligodendrocyte-related dysfunction: a new mechanism of antipsychotics in the treatment of schizophrenia? Int J Neuropsychopharmacol 16 (3), 691-700.

Rentzsch, J., Jockers-Scherubl, M.C., Boutros, N.N., Gallinat, J., 2008. Test-retest reliability of P50, N100 and P200 auditory sensory gating in healthy subjects. Int $\mathrm{J}$ Psychophysiol 67 (2), 81-90.
Rentzsch, J., Penzhorn, A., Kernbichler, K., Plockl, D., Gomez-Carrillo de Castro, A., Gallinat, J., Jockers-Scherubl, M.C., 2007. Differential impact of heavy cannabis use on sensory gating in schizophrenic patients and otherwise healthy controls. Exp Neurol 205 (1), 241-249.

Ringel, T.M., Heidrich, A., Jacob, C.P., Pfuhlmann, B., Stoeber, G., Fallgatter, A.J., 2004. Sensory gating deficit in a subtype of chronic schizophrenic patients. Psychiatry Res 125 (3), 237-245.

Roopun, A.K., Middleton, S.J., Cunningham, M.O., LeBeau, F.E., Bibbig, A., Whittington, M.A., Traub, R.D., 2006. A beta2-frequency $(20-30 \mathrm{~Hz})$ oscillation in nonsynaptic networks of somatosensory cortex. Proc Natl Acad Sci U S A 103 (42), 15646-15650.

Sakowitz, O.W., Schurmann, M., Basar, E., 2000. Oscillatory frontal theta responses are increased upon bisensory stimulation. Clin Neurophysiol 111 (5), 884-893.

Salisbury, D.F., Kuroki, N., Kasai, K., Shenton, M.E., McCarley, R.W., 2007. Progressive and interrelated functional and structural evidence of post-onset brain reduction in schizophrenia. Arch Gen Psychiatry 64 (5), 521-529.

Salisbury, D.F., Shenton, M.E., Sherwood, A.R., Fischer, I.A., Yurgelun-Todd, D.A., Tohen, M., McCarley, R.W., 1998. Firstepisode schizophrenic psychosis differs from first-episode affective psychosis and controls in P300 amplitude over left temporal lobe. Arch Gen Psychiatry 55 (2), 173-180.

Sanchez-Morla, E.M., Garcia-Jimenez, M.A., Barabash, A., Martinez-Vizcaino, V., Mena, J., Cabranes-Diaz, J.A., Baca-Baldomero, E., Santos, J.L., 2008. P50 sensory gating deficit is a common marker of vulnerability 
to bipolar disorder and schizophrenia. Acta Psychiatr Scand 117 (4), 313-318.

Sanchez-Morla, E.M., Santos, J.L., Aparicio, A., Garcia-Jimenez, M.A., Soria, C., Arango, C., 2013. Neuropsychological correlates of P50 sensory gating in patients with schizophrenia. Schizophr Res 143 (1), 102-106.

Sanchez-Morla, E.M., Santos, J.L., Aparicio, A., Garcia-Jimenez, M.A., Villanueva, C., Martinez-Vizcaino, V., Arango, C., 2009. Antipsychotic effects on auditory sensory gating in schizophrenia patients. Eur Neuropsychopharmacol 19 (12), 905-909.

Sandman, C.A., Patterson, J.V., 2000. The auditory event-related potential is a stable and reliable measure in elderly subjects over a 3 year period. Clin Neurophysiol 111 (8), 1427-1437.

Santos, J.L., Sanchez-Morla, E.M., Aparicio, A., Garcia-Jimenez, M.A., Villanueva, C., Martinez-Vizcaino, V., Arango, C., 2010. P50 gating in deficit and nondeficit schizophrenia. Schizophr Res 119 (1-3), 183-190.

Schulze, K.K., Hall, M.H., McDonald, C., Marshall, N., Walshe, M., Murray, R.M., Bramon, E., 2007. P50 auditory evoked potential suppression in bipolar disorder patients with psychotic features and their unaffected relatives. Biol Psychiatry 62 (2), 121-128.

Schulze, K.K., Hall, M.H., McDonald, C., Marshall, N., Walshe, M., Murray, R.M., Bramon, E., 2008. Auditory P300 in patients with bipolar disorder and their unaffected relatives. Bipolar Disord 10 (3), 377-386.

Seese, R.R., O'Neill, J., Hudkins, M., Siddarth, P., Levitt, J., Tseng, B., Wu, K.N., Caplan, R., 2011. Proton magnetic resonance spectroscopy and thought disorder in childhood schizophrenia. Schizophr Res 133 (1-3), 82-90.

Shan, J.C., Liu, C.M., Chiu, M.J., Liu, C.C., Chien, Y.L., Hwang, T.J., Lin, Y.T., Hsieh, M.H., Jaw, F.S., Hwu, H.G., 2013. A diagnostic model incorporating P50 sensory gating and neuropsychological tests for schizophrenia. PLoS One 8 (2), e57197.

Shenton, M.E., Kikinis, R., Jolesz, F.A., Pollak, S.D., LeMay, M., Wible, C.G., Hokama, H., Martin, J., Metcalf, D., Coleman, M., et al., 1992. Abnormalities of the left temporal lobe and thought disorder in schizophrenia. A quantitative magnetic resonance imaging study. N Engl J Med 327 (9), 604-612.

Shinn, A.K., Baker, J.T., Cohen, B.M., Ongur, D., 2013. Functional connectivity of left Heschl's gyrus in vulnerability to auditory hallucinations in schizophrenia. Schizophr Res 143 (2-3), 260-268.

Siegel, C., Waldo, M., Mizner, G., Adler, L.E., Freedman, R., 1984. Deficits in sensory gating in schizophrenic patients and their relatives. Evidence obtained with auditory evoked responses. Arch Gen Psychiatry 41 (6), 607-612.

Sivarao, D.V., 2015. The 40-Hz auditory steady-state response: a selective biomarker for cortical NMDA function. Ann N Y Acad Sci 1344, 27-36.

Smith, A.K., Edgar, J.C., Huang, M., Lu, B.Y., Thoma, R.J., Hanlon, F.M., McHaffie, G., Jones, A.P., Paz, R.D., Miller, G.A., Canive, J.M., 2010. Cognitive abilities and 50- and $100-\mathrm{msec}$ paired-click processes in schizophrenia. Am J Psychiatry 167 (10), 1264-1275.

Smith, D.M., Grant, B., Fisher, D.J., Borracci, G., Labelle, A., Knott, V.J., 2013. Auditory verbal hallucinations in schizophrenia correlate with P50 gating. Clin Neurophysiol 124 (7), 1329-1335. 
Smucny, J., Olincy, A., Eichman, L.C., Lyons, E., Tregellas, J.R., 2013. Early sensory processing deficits predict sensitivity to distraction in schizophrenia. Schizophr Res 147 (1), 196-200.

Song, L., Chen, X., Chen, M., Tang, Y., Wang, J., Zhang, M., Lou, F., Liang, J., Chen, C., 2014. Differences in P50 and prepulse inhibition of the startle reflex between male smokers and non-smokers with first episode schizophrenia without medical treatment. Chin Med J (Engl) 127 (9), 1651-1655.

StataCorp., 2017. Stata Statistical Software, Release 15 ed. Stata Corp LLC, College Station, TX.

Stelzer, A., Kay, A.R., Wong, R.K., 1988. GABAA-receptor function in hippocampal cells is maintained by phosphorylation factors. Science 241 (4863), 339-341.

Storozheva, Z.I., Kirenskaya, A.V., Novototsky-Vlasov, V.Y., Telesheva, K.Y., Pletnikov, M., 2016. Startle Modification and P50 Gating in Schizophrenia Patients and Controls: Russian Population. Span J Psychol 19, E8.

Strakowski, S.M., Delbello, M.P., Adler, C.M., 2005. The functional neuroanatomy of bipolar disorder: a review of neuroimaging findings. Mol Psychiatry 10 (1), 105-116.

Stroup, D.F., Berlin, J.A., Morton, S.C., Olkin, I., Williamson, G.D., Rennie, D., Moher, D., Becker, B.J., Sipe, T.A., Thacker, S.B., 2000. Meta-analysis of observational studies in epidemiology: a proposal for reporting. Meta-analysis Of Observational Studies in Epidemiology (MOOSE) group. JAMA 283 (15), 2008-2012.

Swann, A.C., Lijffijt, M., Lane, S.D., Steinberg, J.L., Acas, M.D., Cox, B., Moeller, F.G., 2013. Pre-attentive information processing and impulsivity in bipolar disorder. $\mathbf{J}$ Psychiatr Res 47 (12), 1917-1924.
Tamminga, C.A., Ivleva, E.I., Keshavan, M.S., Pearlson, G.D., Clementz, B.A., Witte, B., Morris, D.W., Bishop, J., Thaker, G.K., Sweeney, J.A., 2013. Clinical phenotypes of psychosis in the Bipolar-Schizophrenia Network on Intermediate Phenotypes (BSNIP). Am J Psychiatry 170 (11), 12631274.

Tan, D., Ozerdem, A., Guntekin, B., Atagun, M.I., Tulay, E., Karadag, F., Basar, E., 2016. Increased Beta Frequency $(15-30 \mathrm{~Hz})$ Oscillatory Responses in Euthymic Bipolar Patients Under Lithium Monotherapy. Clin EEG Neurosci 47 (2), 87-95.

Tang, Y., Wang, J., Zhang, T., Xu, L., Qian, Z., Cui, H., Tang, X., Li, H., Whitfield-Gabrieli, S., Shenton, M.E., Seidman, L.J., McCarley, R.W., Keshavan, M.S., Stone, W.S., Wang, J., Niznikiewicz, M.A., 2019. P300 as an index of transition to psychosis and of remission: Data from a clinical high risk for psychosis study and review of literature. Schizophr Res.

Thoma, R.J., Hanlon, F.M., Moses, S.N., Edgar, J.C., Huang, M., Weisend, M.P., Irwin, J., Sherwood, A., Paulson, K., Bustillo, J., Adler, L.E., Miller, G.A., Canive, J.M., 2003. Lateralization of auditory sensory gating and neuropsychological dysfunction in schizophrenia. Am J Psychiatry 160 (9), 1595-1605.

Thoma, R.J., Hanlon, F.M., Moses, S.N., Ricker, D., Huang, M., Edgar, C., Irwin, J., Torres, F., Weisend, M.P., Adler, L.E., Miller, G.A., Canive, J.M., 2005. M50 sensory gating predicts negative symptoms in schizophrenia. Schizophr Res 73 (2-3), 311-318.

Thoma, R.J., Hanlon, F.M., Petropoulos, H., Miller, G.A., Moses, S.N., Smith, A., Parks, L., Lundy, S.L., Sanchez, N.M., Jones, A., Huang, M., Weisend, M.P., Canive, J.M., 2008. Schizophrenia diagnosis and anterior hippocampal volume make separate 
contributions to sensory gating. Psychophysiology 45 (6), 926-935.

Thoma, R.J., Hanlon, F.M., Sanchez, N., Weisend, M.P., Huang, M., Jones, A., Miller, G.A., Canive, J.M., 2004. Auditory sensory gating deficit and cortical thickness in schizophrenia. Neurol Clin Neurophysiol 2004, 62.

Torres, I.J., Kozicky, J., Popuri, S., Bond, D.J., Honer, W.G., Lam, R.W., Yatham, L.N., 2014. 12-month longitudinal cognitive functioning in patients recently diagnosed with bipolar disorder. Bipolar Disord 16 (2), 159-171.

Toyomaki, A., Hashimoto, N., Kako, Y., Tomimatsu, Y., Koyama, T., Kusumi, I., 2015. Different P50 sensory gating measures reflect different cognitive dysfunctions in schizophrenia. Schizophr Res Cogn 2 (3), 166-169.

Traub, R.D., Bibbig, A., LeBeau, F.E., Buhl, E.H., Whittington, M.A., 2004. Cellular mechanisms of neuronal population oscillations in the hippocampus in vitro. Annu Rev Neurosci 27, 247-278.

Trotta, A., Murray, R.M., MacCabe, J.H., 2015. Do premorbid and post-onset cognitive functioning differ between schizophrenia and bipolar disorder? A systematic review and meta-analysis. Psychol Med 45 (2), 381394.

Turetsky, B.I., Bilker, W.B., Siegel, S.J., Kohler, C.G., Gur, R.E., 2009. Profile of auditory information-processing deficits in schizophrenia. Psychiatry Res 165 (1-2), 2737.

Ucar, E., Lehtinen, E.K., Glenthoj, B.Y., Oranje, B., 2012. The effect of acute exogenous melatonin on P50 suppression in healthy male volunteers stratified for low and high gating levels. J Psychopharmacol 26 (8), 1113-1118.
Uhlhaas, P.J., Singer, W., 2006. Neural synchrony in brain disorders: relevance for cognitive dysfunctions and pathophysiology. Neuron 52 (1), 155-168.

Varela, F., Lachaux, J.P., Rodriguez, E., Martinerie, J., 2001. The brainweb: phase synchronization and large-scale integration. Nat Rev Neurosci 2 (4), 229-239.

Venables, P.H., 1964. Input Dysfunction in Schizophrenia. Prog Exp Pers Res 72, 1-47.

Vinogradov, S., Solomon, S., Ober, B.A., Biggins, C.A., Shenaut, G.K., Fein, G., 1996. Do semantic priming effects correlate with sensory gating in schizophrenia? Biol Psychiatry 39 (9), 821-824.

Vita, A., De Peri, L., Deste, G., Barlati, S., Sacchetti, E., 2015. The Effect of Antipsychotic Treatment on Cortical Gray Matter Changes in Schizophrenia: Does the Class Matter? A Meta-analysis and Metaregression of Longitudinal Magnetic Resonance Imaging Studies. Biol Psychiatry 78 (6), 403-412.

Vita, A., De Peri, L., Deste, G., Sacchetti, E., 2012. Progressive loss of cortical gray matter in schizophrenia: a meta-analysis and meta-regression of longitudinal MRI studies. Transl Psychiatry 2, e190.

von Stein, A., Sarnthein, J., 2000. Different frequencies for different scales of cortical integration: from local gamma to long range alpha/theta synchronization. Int $\mathrm{J}$ Psychophysiol 38 (3), 301-313.

Vuillier, L., Hermens, D.F., Chitty, K., Wang, C., Kaur, M., Ward, P.B., Degabriele, R., Hickie, I.B., Lagopoulos, J., 2015. Emotional processing, p50 sensory gating, and social functioning in bipolar disorder. Clin EEG Neurosci 46 (2), 81-87.

Wagner, A.D., Schacter, D.L., Rotte, M., Koutstaal, W., Maril, A., Dale, A.M., Rosen, 
B.R., Buckner, R.L., 1998. Building memories: remembering and forgetting of verbal experiences as predicted by brain activity. Science 281 (5380), 1188-1191.

Waldo, M., Gerhardt, G., Baker, N., Drebing, C., Adler, L., Freedman, R., 1992. Auditory sensory gating and catecholamine metabolism in schizophrenic and normal subjects. Psychiatry Res 44 (1), 21-32.

Waldo, M.C., Adler, L.E., Freedman, R., 1988. Defects in auditory sensory gating and their apparent compensation in relatives of schizophrenics. Schizophr Res 1 (1), 19-24.

Waldo, M.C., Cawthra, E., Adler, L.E., Dubester, S., Staunton, M., Nagamoto, H., Baker, N., Madison, A., Simon, J., Scherzinger, A., et al., 1994. Auditory sensory gating, hippocampal volume, and catecholamine metabolism in schizophrenics and their siblings. Schizophr Res 12 (2), 93-106.

Waldo, M.C., Woodward, L., Adler, L.E., 2010. Varenicline and P50 auditory gating in medicated schizophrenic patients: a pilot study. Psychiatry Res 175 (1-2), 179-180.

Wang, Y., Feng, Y., Jia, Y., Wang, W., Xie, Y., Guan, Y., Zhong, S., Zhu, D., Huang, L., 2014a. Auditory M50 and M100 sensory gating deficits in bipolar disorder: a MEG study. J Affect Disord 152-154, 131-138.

Wang, Y., Jia, Y., Feng, Y., Zhong, S., Xie, Y., Wang, W., Guan, Y., Zhu, D., Huang, L., 2014b. Overlapping auditory M100 and M200 abnormalities in schizophrenia and bipolar disorder: a MEG study. Schizophr Res 160 (1-3), 201-207.

Waters, F., Price, G., Dragovic, M., Jablensky, A., 2009. Electrophysiological brain activity and antisaccade performance in schizophrenia patients with first-rank (passivity) symptoms. Psychiatry Res 170 (2-3), 140-149.
Whittington, M.A., Faulkner, H.J., Doheny, H.C., Traub, R.D., 2000. Neuronal fast oscillations as a target site for psychoactive drugs. Pharmacol Ther 86 (2), 171-190.

Williams, T.J., Nuechterlein, K.H., Subotnik, K.L., Yee, C.M., 2011. Distinct neural generators of sensory gating in schizophrenia. Psychophysiology 48 (4), 470-478.

Wittchen, H.U., Jacobi, F., Rehm, J., Gustavsson, A., Svensson, M., Jonsson, B., Olesen, J., Allgulander, C., Alonso, J., Faravelli, C., Fratiglioni, L., Jennum, P., Lieb, R., Maercker, A., van Os, J., Preisig, M., Salvador-Carulla, L., Simon, R., Steinhausen, H.C., 2011. The size and burden of mental disorders and other disorders of the brain in Europe 2010. Eur Neuropsychopharmacol 21 (9), 655-679.

Witten, L., Bastlund, J.F., Glenthoj, B.Y., Bundgaard, C., Steiniger-Brach, B., Mork, A., Oranje, B., 2016. Comparing Pharmacological Modulation of Sensory Gating in Healthy Humans and Rats: The Effects of Reboxetine and Haloperidol. Neuropsychopharmacology 41 (2), 638-645.

Wonodi, I., McMahon, R.P., Krishna, N., Mitchell, B.D., Liu, J., Glassman, M., Hong, L.E., Gold, J.M., 2014. Influence of kynurenine 3-monooxygenase (KMO) gene polymorphism on cognitive function in schizophrenia. Schizophr Res. 160 (1-3), 8087.

Yee, C.M., Nuechterlein, K.H., Morris, S.E., White, P.M., 1998. P50 suppression in recent-onset schizophrenia: clinical correlates and risperidone effects. J Abnorm Psychol. 107 (4), 691-698.

Yee, C.M., Williams, T.J., White, P.M., Nuechterlein, K.H., Ames, D., Subotnik, K.L., 2010. Attentional modulation of the P50 suppression deficit in recent-onset and 
chronic schizophrenia. J Abnorm Psychol. 119 (1), 31-39.

Yener, G., Guntekin, B., Basar, E., 2008. Eventrelated delta oscillatory responses of Alzheimer patients. Eur J Neurol. 15 (6), 540-547.

Yener, G.G., Guntekin, B., Orken, D.N., Tulay, E., Forta, H., Basar, E., 2012. Auditory delta event-related oscillatory responses are decreased in Alzheimer's disease. Behav Neurol. 25 (1), 3-11.

Yildiz-Yesiloglu, A., Ankerst, D.P., 2006. Neurochemical alterations of the brain in bipolar disorder and their implications for pathophysiology: a systematic review of the in vivo proton magnetic resonance spectroscopy findings. Prog Neuropsychopharmacol Biol Psychiatry. 30 (6), 969-995.

Yoshimura, M., Koenig, T., Irisawa, S., Isotani, T., Yamada, K., Kikuchi, M., Okugawa, G., Yagyu, T., Kinoshita, T., Strik, W., Dierks, T., 2007. A pharmaco-EEG study on antipsychotic drugs in healthy volunteers. Psychopharmacology (Berl) 191 (4), 9951004.

Yuksel, C., Ongur, D., 2010. Magnetic resonance spectroscopy studies of glutamate-related abnormalities in mood disorders. Biol Psychiatry. 68 (9), 785-794.

Zanelli, J., Reichenberg, A., Morgan, K., Fearon, P., Kravariti, E., Dazzan, P., Morgan, C., Zanelli, C., Demjaha, A., Jones, P.B., Doody, G.A., Kapur, S., Murray, R.M., 2010. Specific and generalized neuropsychological deficits: a comparison of patients with various first-episode psychosis presentations. Am J Psychiatry. 167 (1), 78-85.

Zhang, J., Abdallah, C.G., Wang, J., Wan, X., Liang, C., Jiang, L., Liu, Y., Huang, H., Hong, X., Huang, Q., Wu, R., Xu, C., 2012. Upregulation of adenosine A2A receptors induced by atypical antipsychotics and its correlation with sensory gating in schizophrenia patients. Psychiatry Res. 200 (2-3), 126-132.

\section{Supplementary Material}

Table S1. Eggers' Test bias per outcome per disease group

\begin{tabular}{|l|c|c|}
\hline & Bias coefficient & p-value \\
\hline a. S1 amplitudes, schizophrenia & $\mathbf{3 . 5 9}$ & $<\mathbf{0 . 0 0 1}$ \\
\hline b. S1 amplitudes, bipolar disorder & 5.28 & 0.069 \\
\hline c. S1 amplitudes, healthy controls & $\mathbf{5 . 8 6}$ & $<\mathbf{0 . 0 0 1}$ \\
\hline c1. S1 amplitudes, relatives & $\mathbf{- 4 . 2 2}$ & $\mathbf{0 . 0 8 9}$ \\
\hline d. S2 amplitudes, schizophrenia & $\mathbf{3 . 8 7}$ & $<\mathbf{0 . 0 0 1}$ \\
\hline e. S2 amplitudes, bipolar disorder & 2.94 & 0.089 \\
\hline f. S2 amplitudes, healthy controls & $\mathbf{4 . 1 4}$ & $<\mathbf{0 . 0 0 1}$ \\
\hline f1. S2 amplitudes, relatives & 1.19 & 0.47 \\
\hline g. S2/S1 ratio, schizophrenia & 0.86 & 0.12 \\
\hline h. S2/S1 ratio, bipolar disorder & 3.36 & 0.10 \\
\hline i. S2/S1 ratio, healthy controls & $\mathbf{2 . 4 2}$ & $\mathbf{0 . 0 2 9}$ \\
\hline i1. S2/S1 ratio, relative & -1.23 & 0.27 \\
\hline j. S1- S2 difference, schizophrenia & $\mathbf{1 . 8 7}$ & $\mathbf{0 . 0 1 3}$ \\
\hline k. S1- S2 difference, bipolar disorder & 2.93 & 0.21 \\
\hline l. S1- S2 difference, healthy controls & $\mathbf{5 . 0 2}$ & $\mathbf{0 . 0 0 1}$ \\
\hline 11 S1- S2 difference, relatives & -0.36 & 0.94 \\
\hline
\end{tabular}




\section{Figure S2. Funnel plots}

a. S1 amplitudes (Schizophrenia)

Funnel plot with pseudo $95 \%$ confidence limits

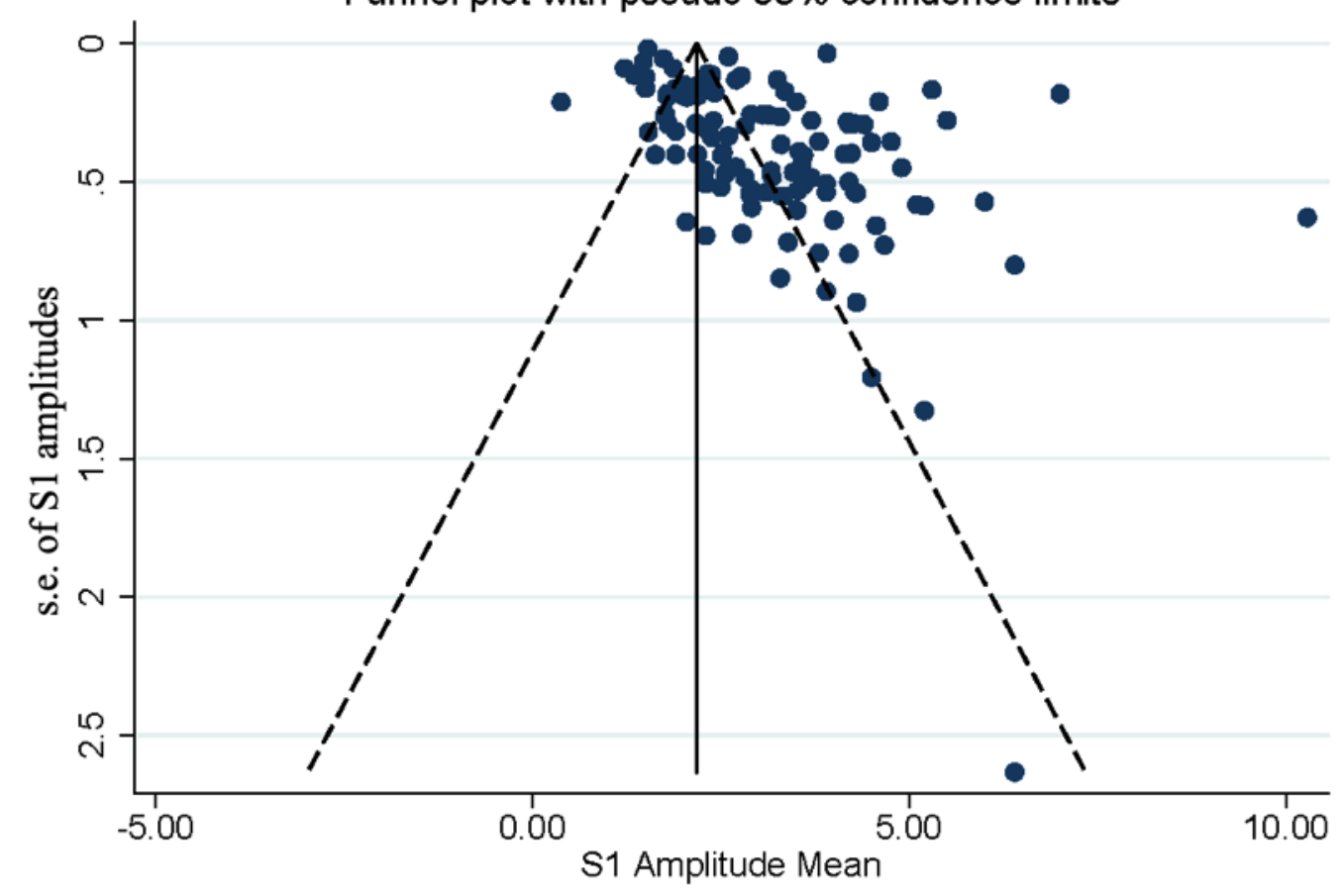

b. S1 amplitudes (Bipolar disorder)

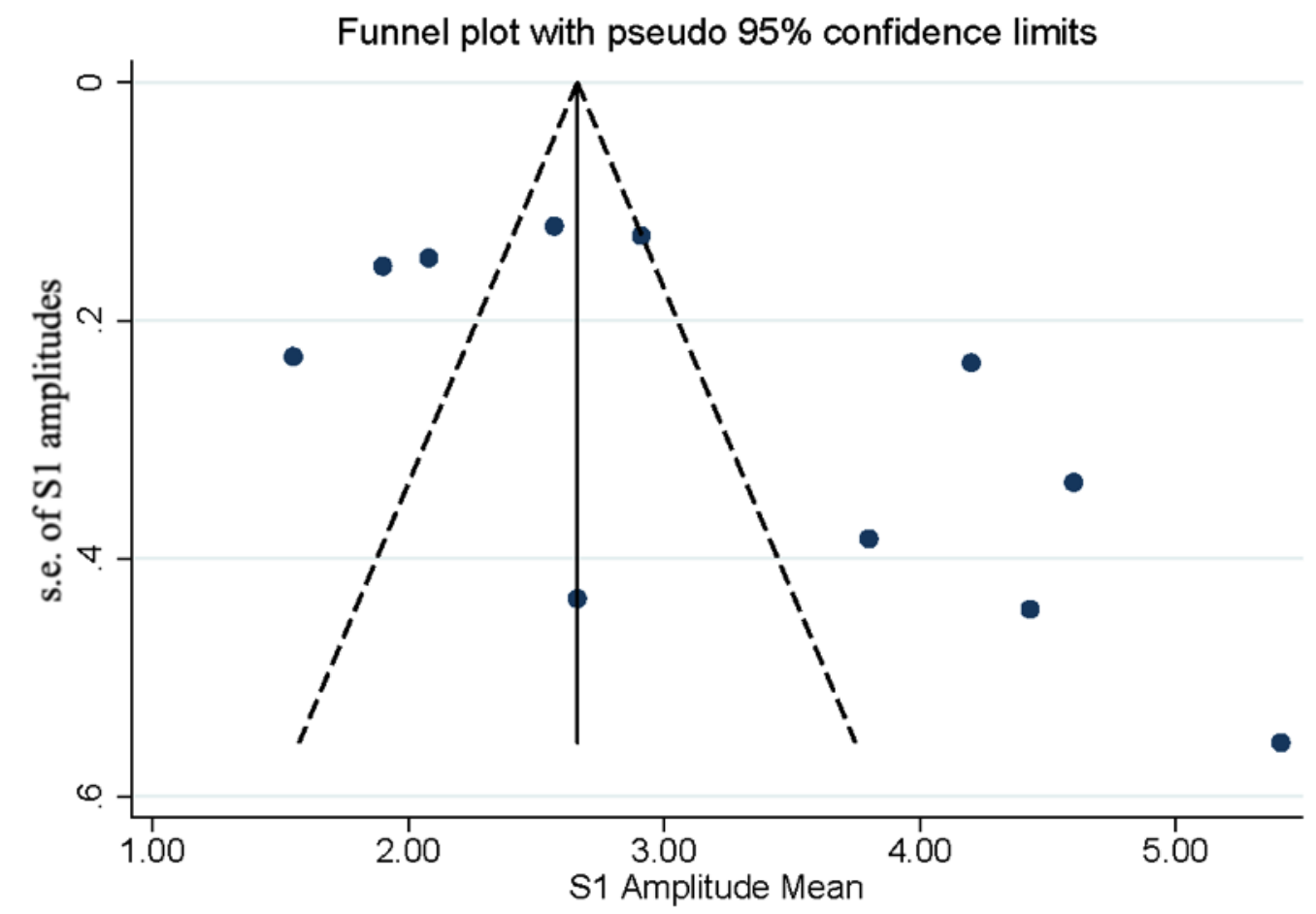


c. S1 amplitudes (Healthy controls)

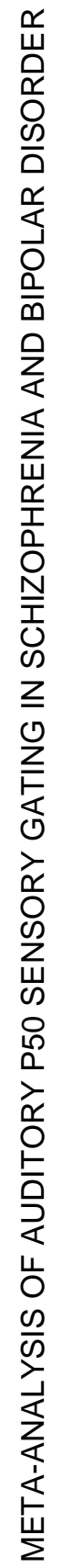

Funnel plot with pseudo $95 \%$ confidence limits

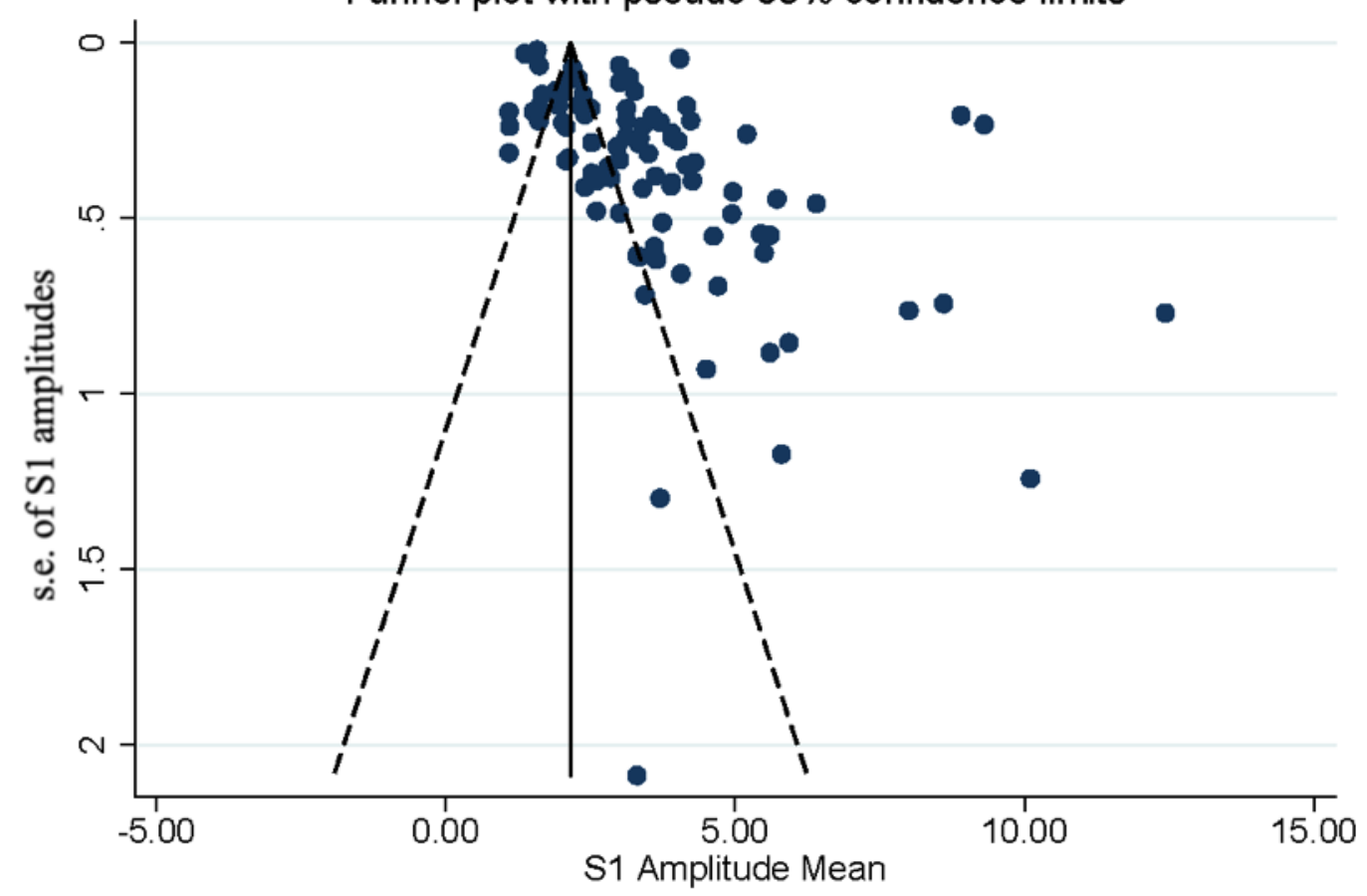

d. S1 amplitudes (First-degree relatives)

Funnel plot with pseudo $95 \%$ confidence limits

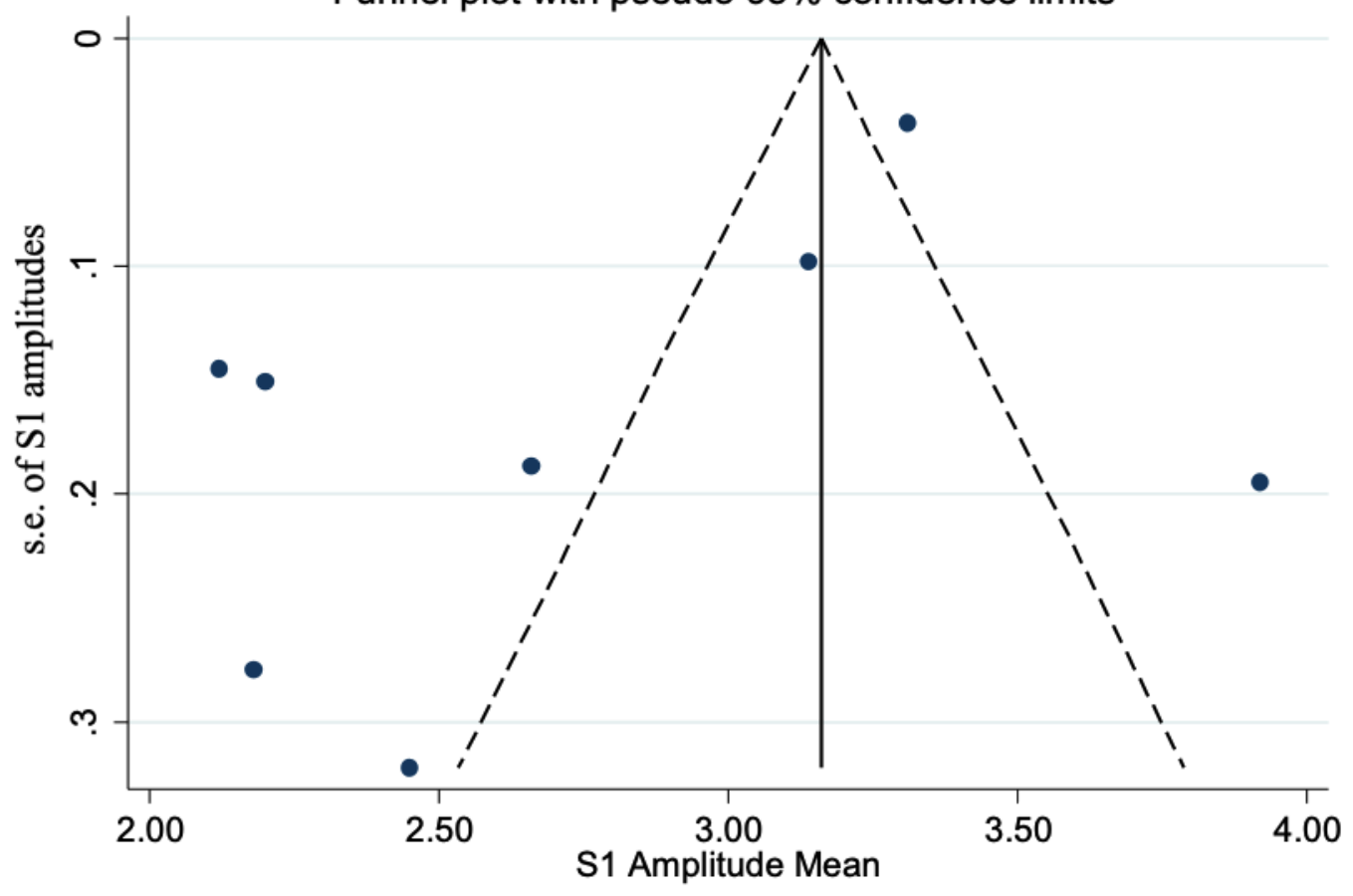


e. S2 amplitudes (Schizophrenia)

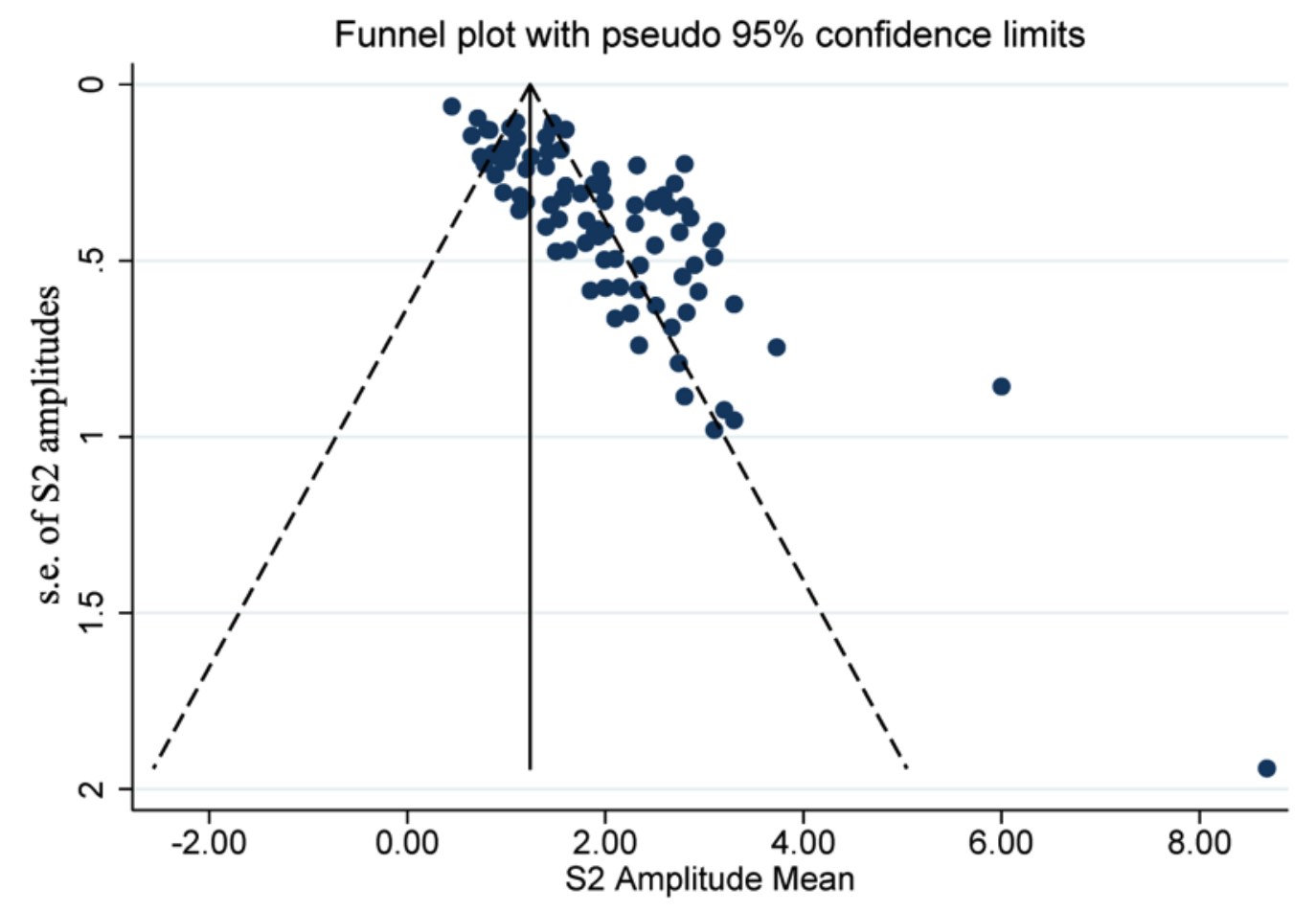

f. S2 amplitudes (Bipolar disorder)

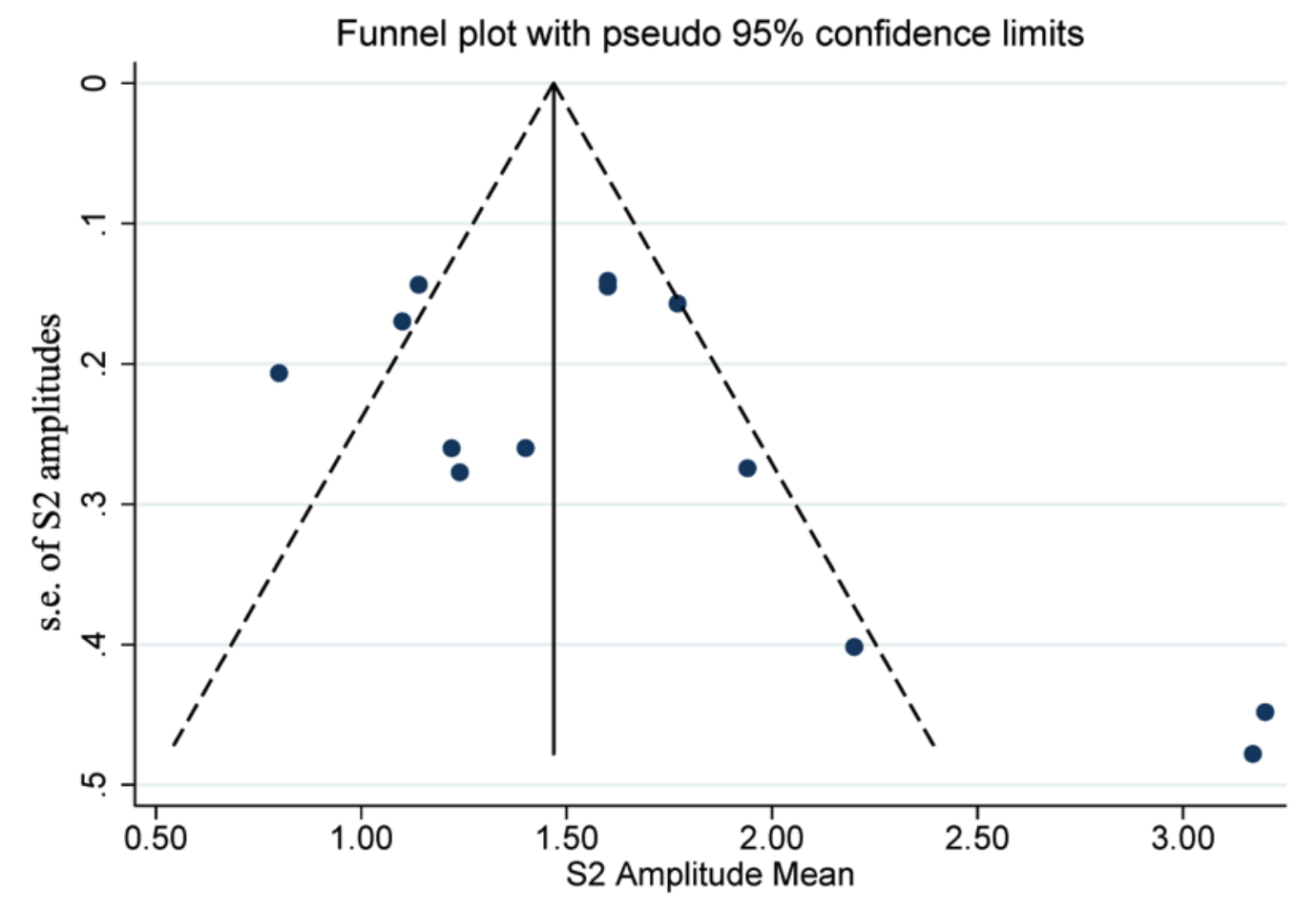


g. S2 amplitudes (Healthy controls)

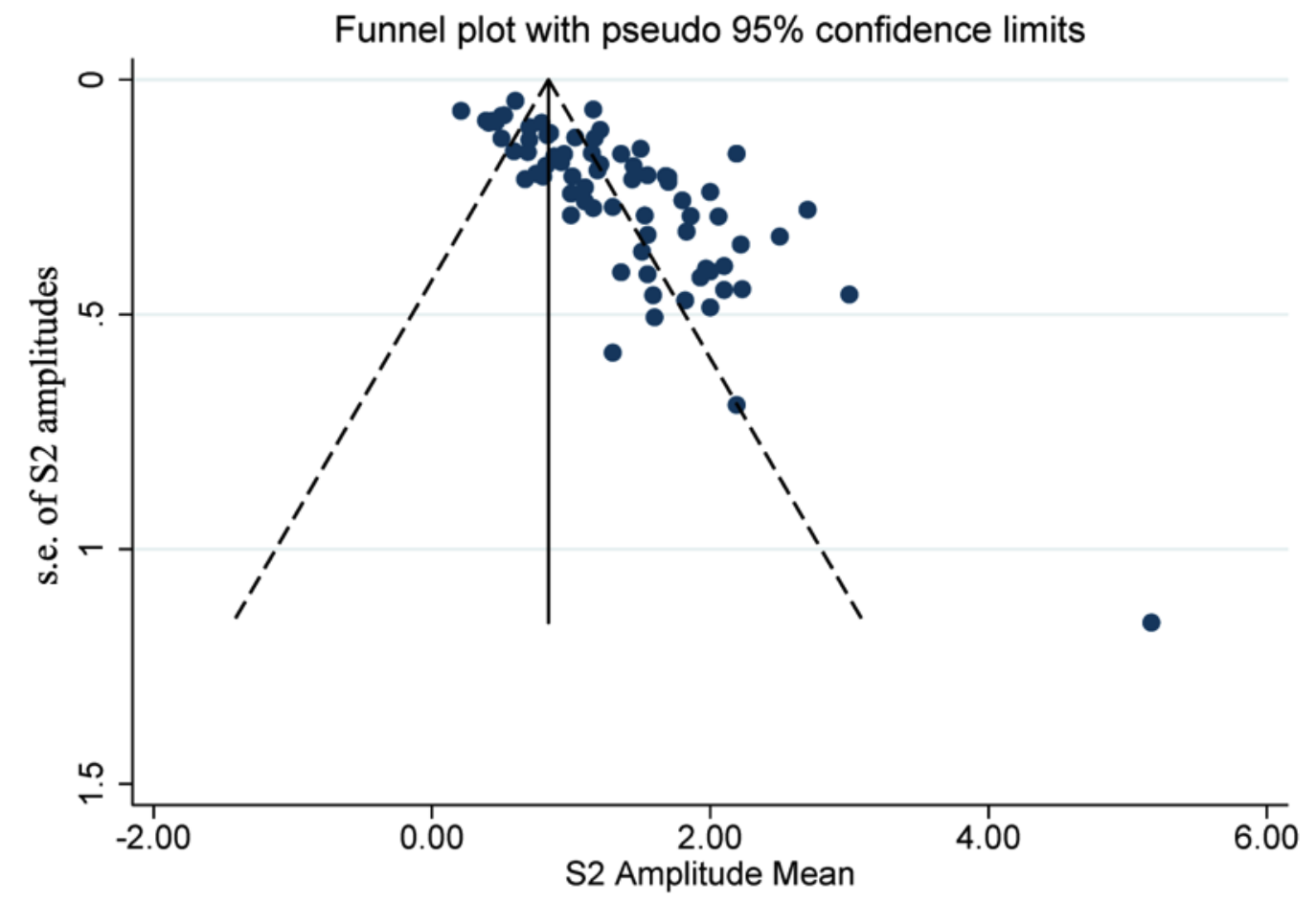

h. S2 amplitudes (First-degree relatives)

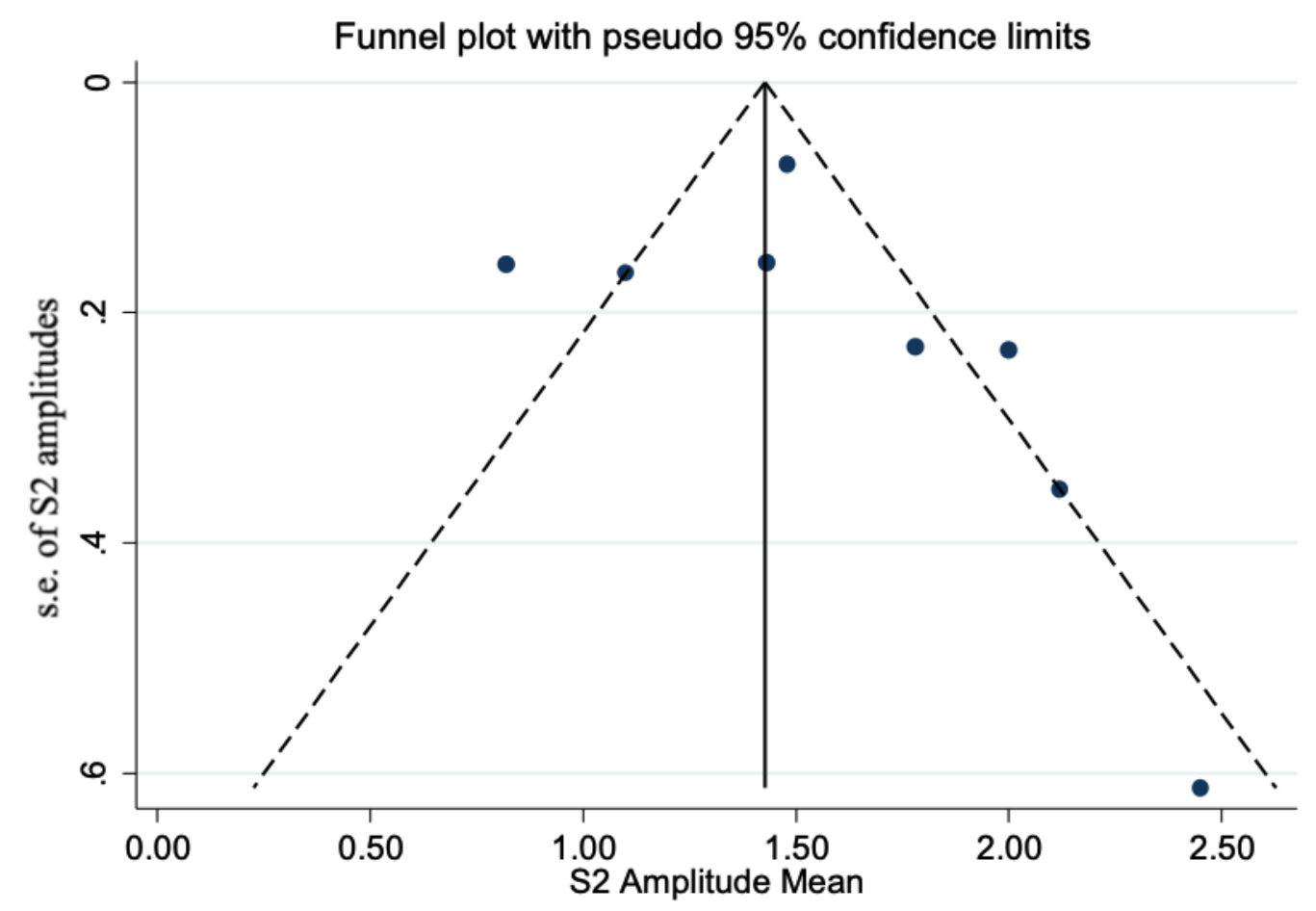


i. S2/S1 ratio (Schizophrenia)

Funnel plot with pseudo $95 \%$ confidence limits

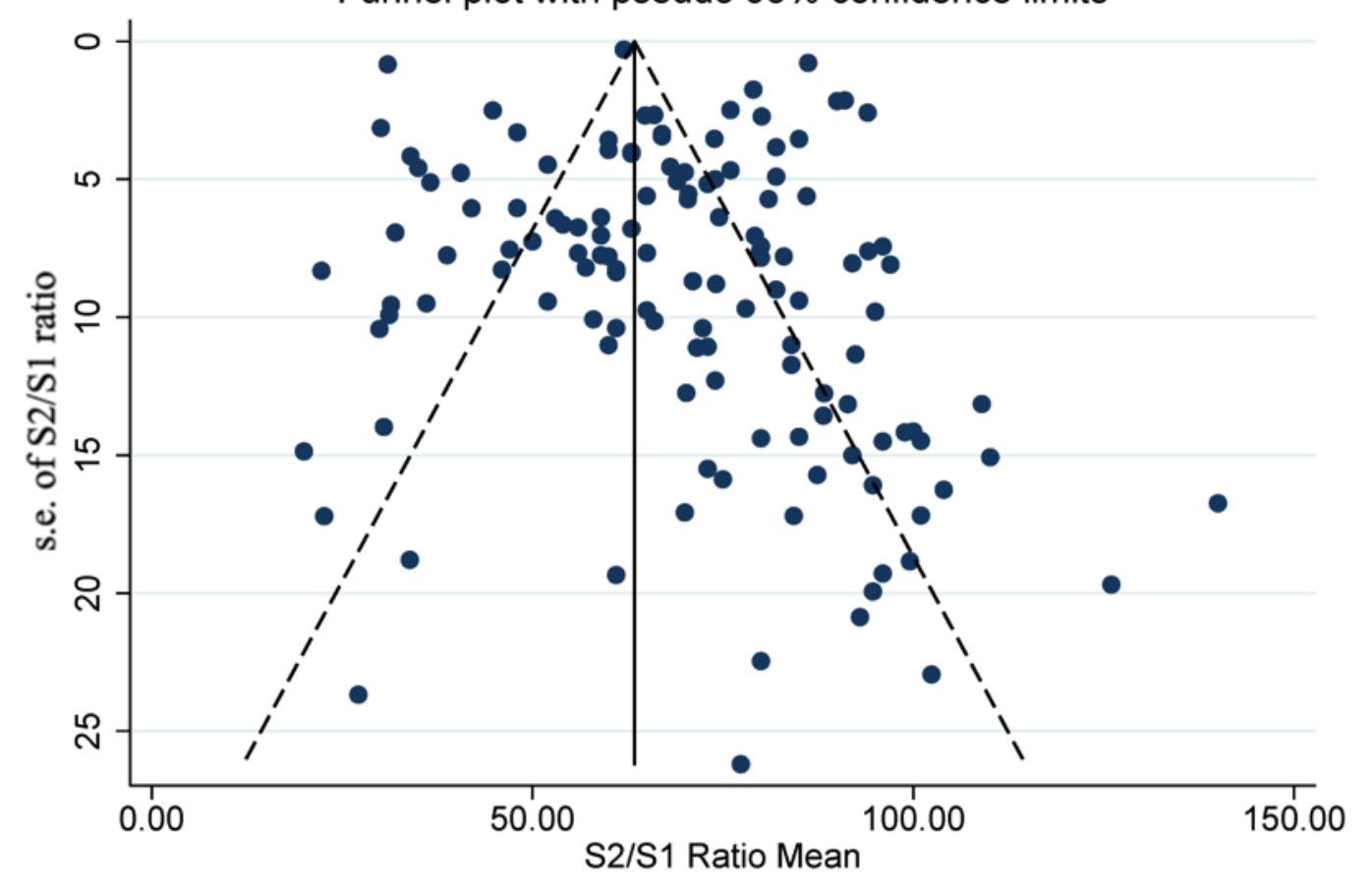

j. S2/S1 ratio (Bipolar disorder)

Funnel plot with pseudo $95 \%$ confidence limits

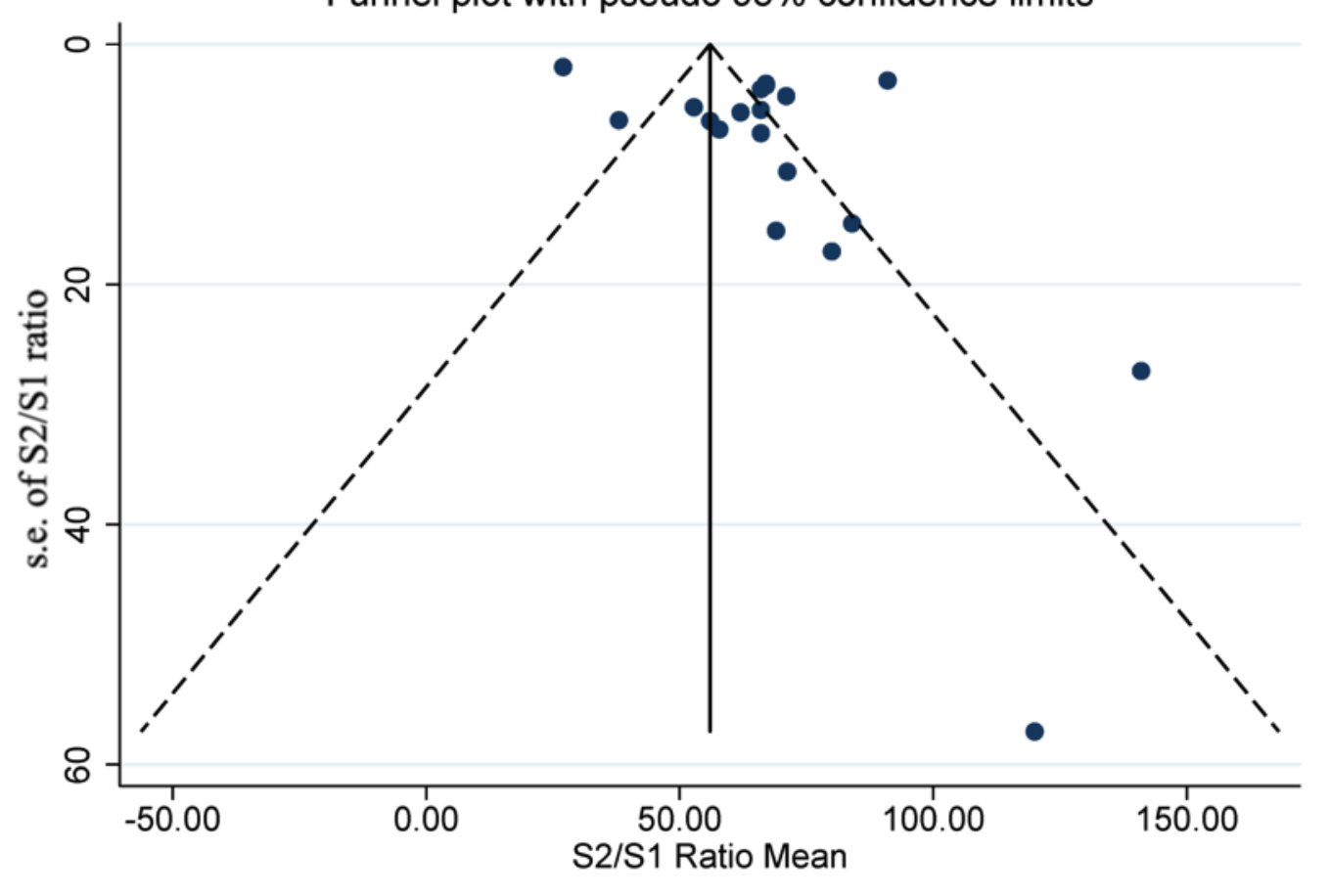


k. S2/S1 ratio (Healthy controls)

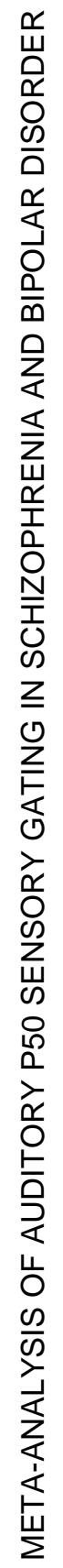

Funnel plot with pseudo $95 \%$ confidence limits

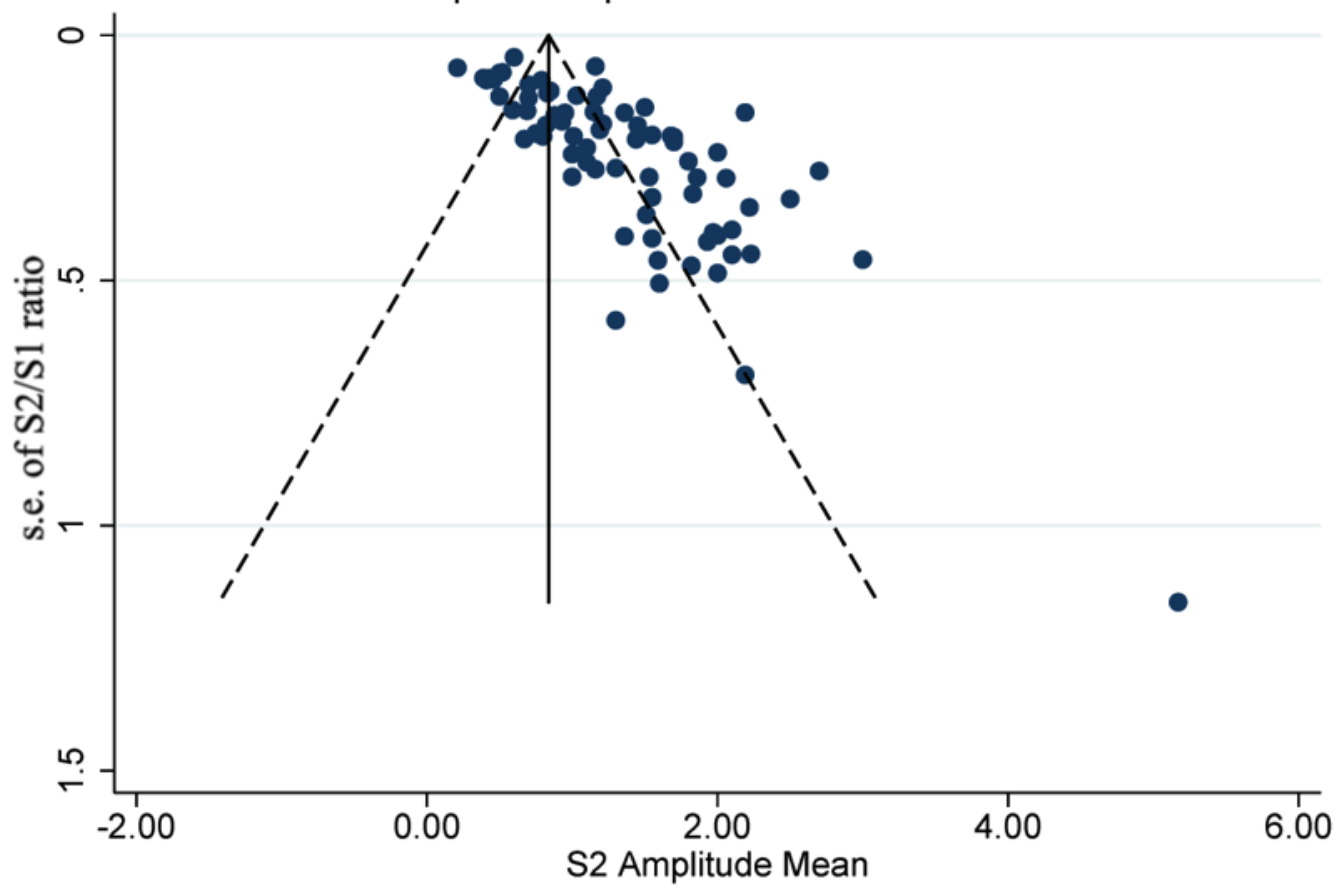

1. S2/S1 ratio (First-degree relatives)

Funnel plot with pseudo $95 \%$ confidence limits

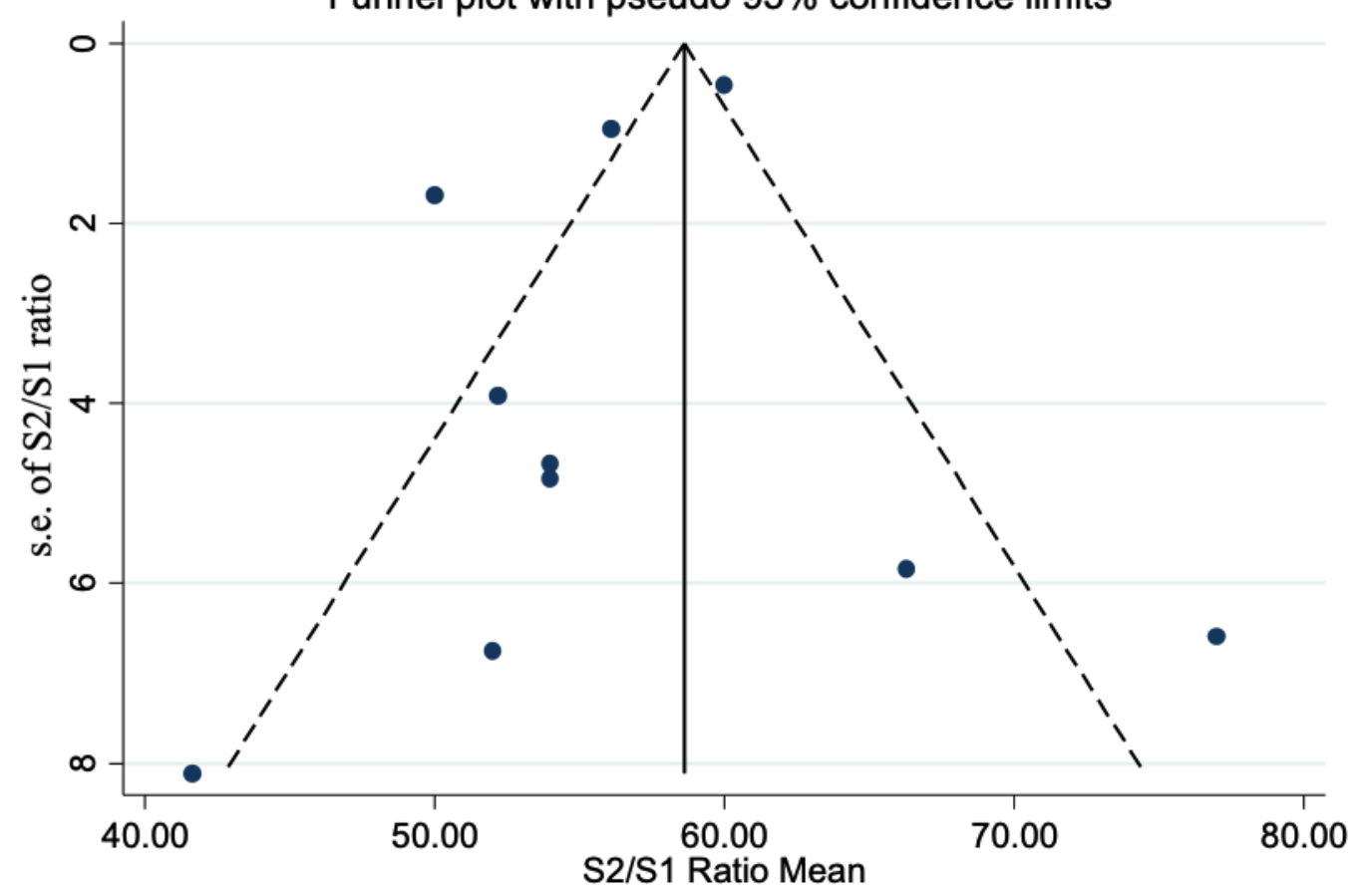


m. S1- S2 difference (Schizophrenia)

Funnel plot with pseudo $95 \%$ confidence limits

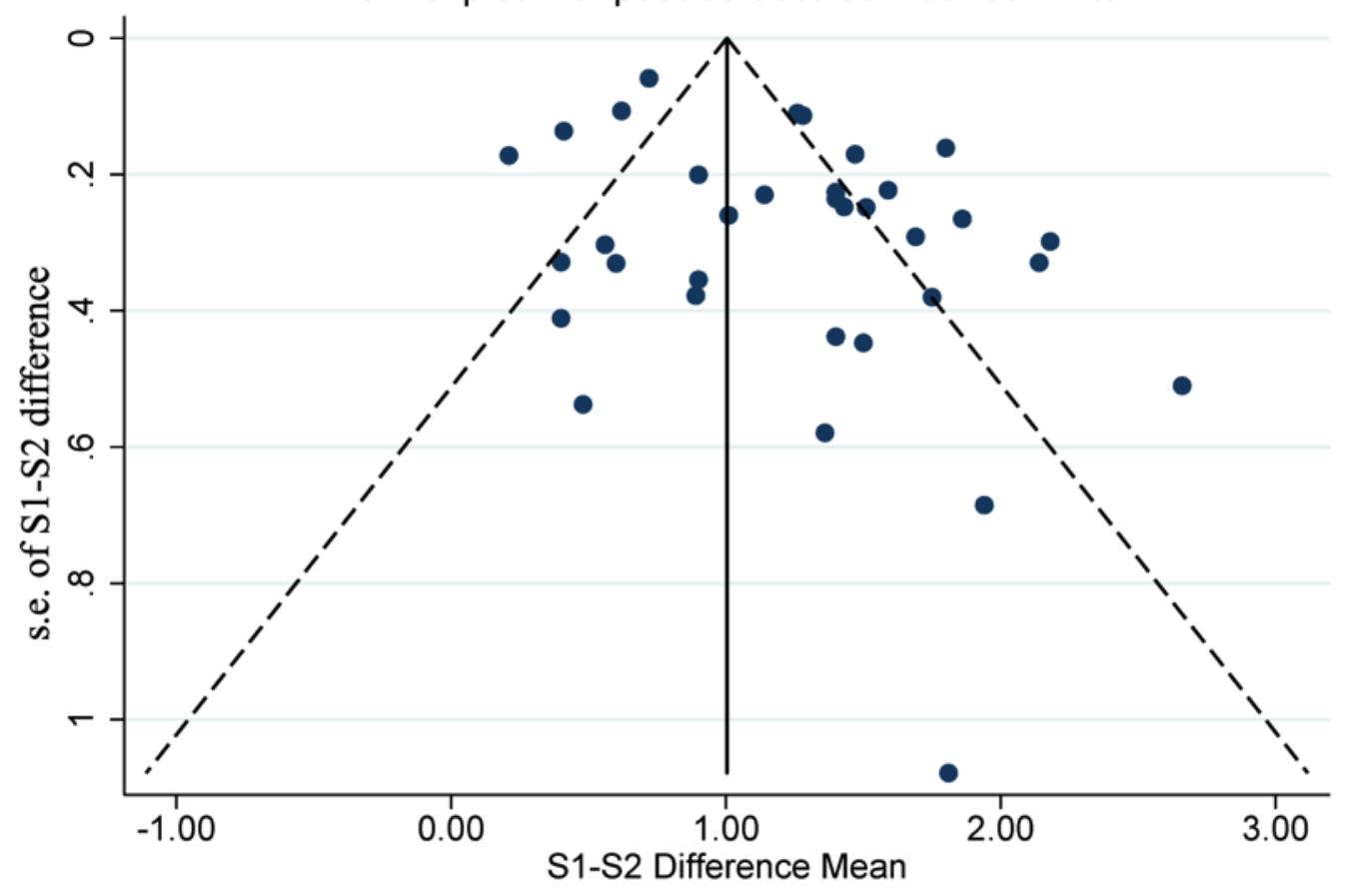

n. S1- S2 difference (Bipolar disorder)

Funnel plot with pseudo $95 \%$ confidence limits

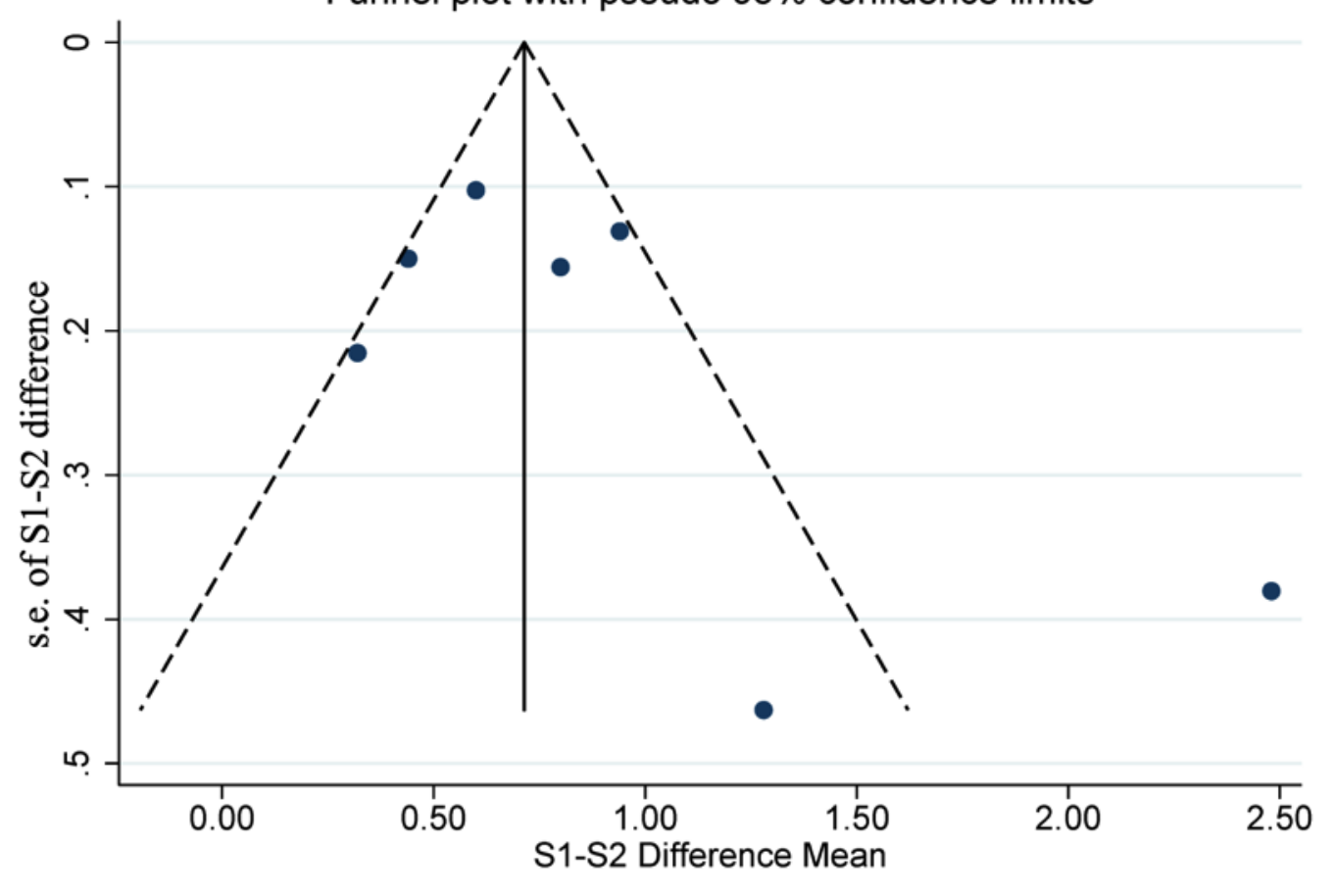


o. S1-S2 difference (Healthy controls)

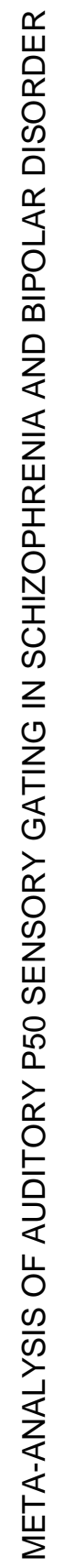

Funnel plot with pseudo $95 \%$ confidence limits

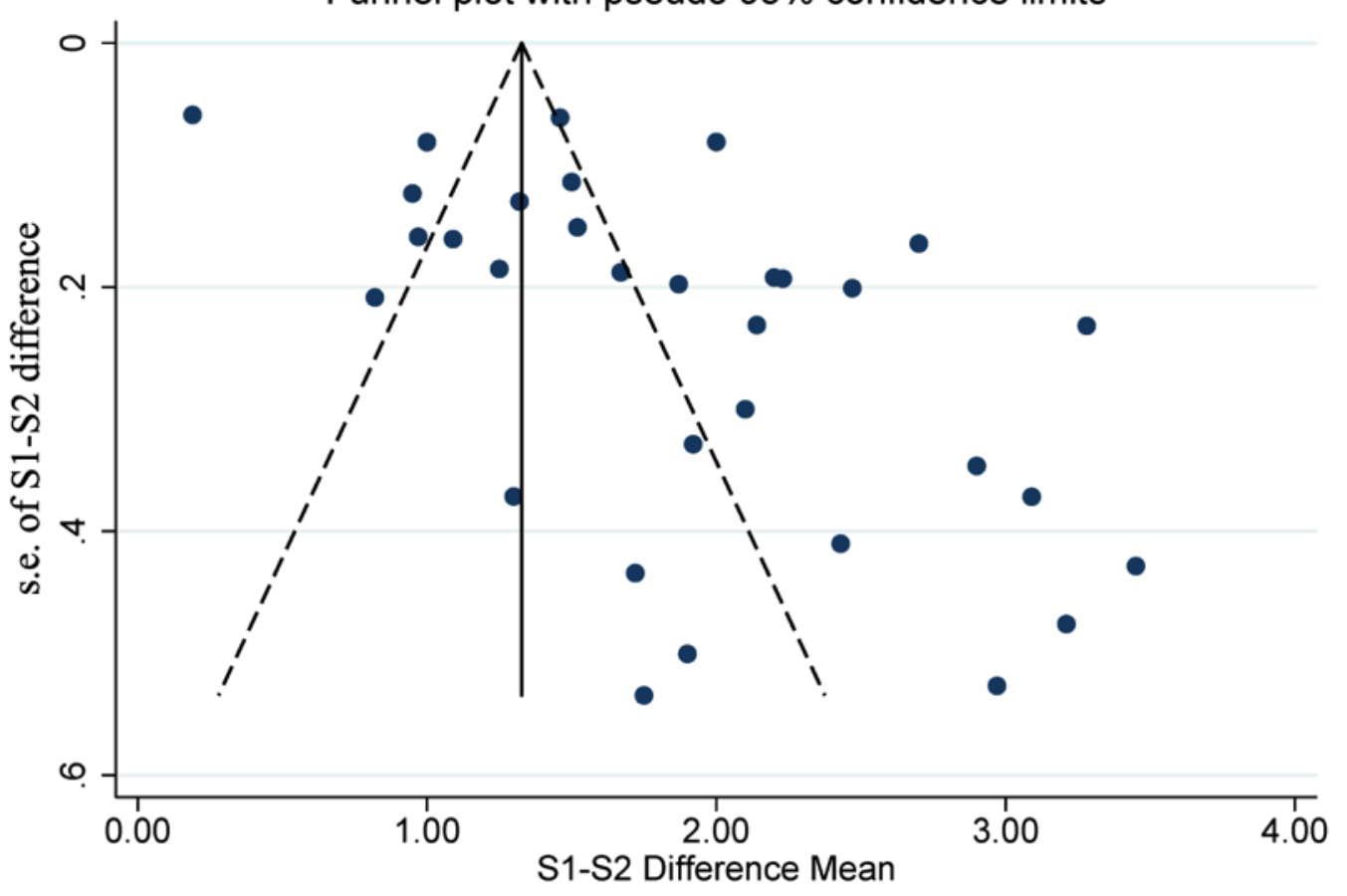

p. S1-S2 difference (First-degree relatives)

Funnel plot with pseudo $95 \%$ confidence limits

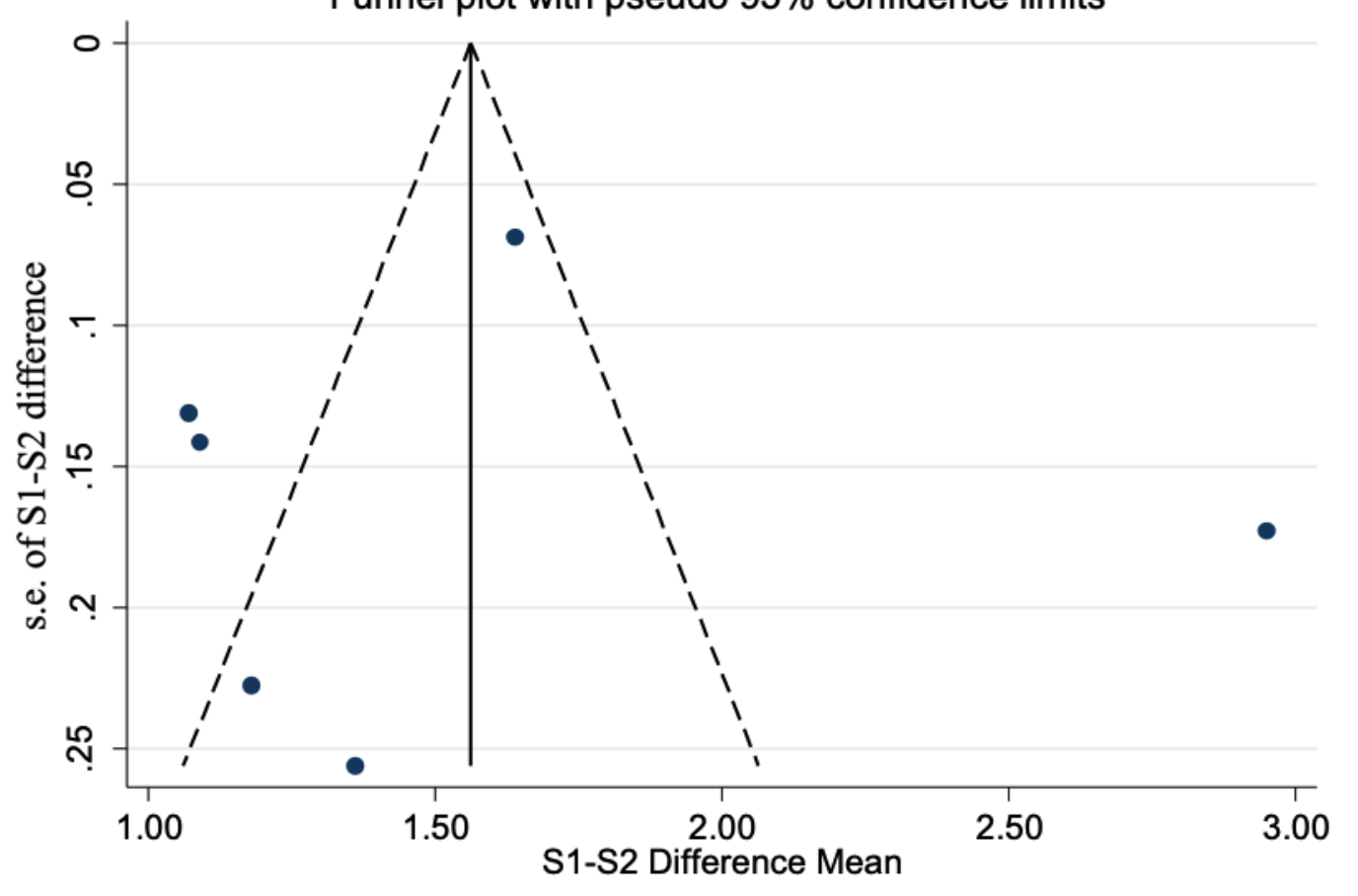


Figure S3. Trim and fill Analysis

a. S1 amplitudes (Schizophrenia)

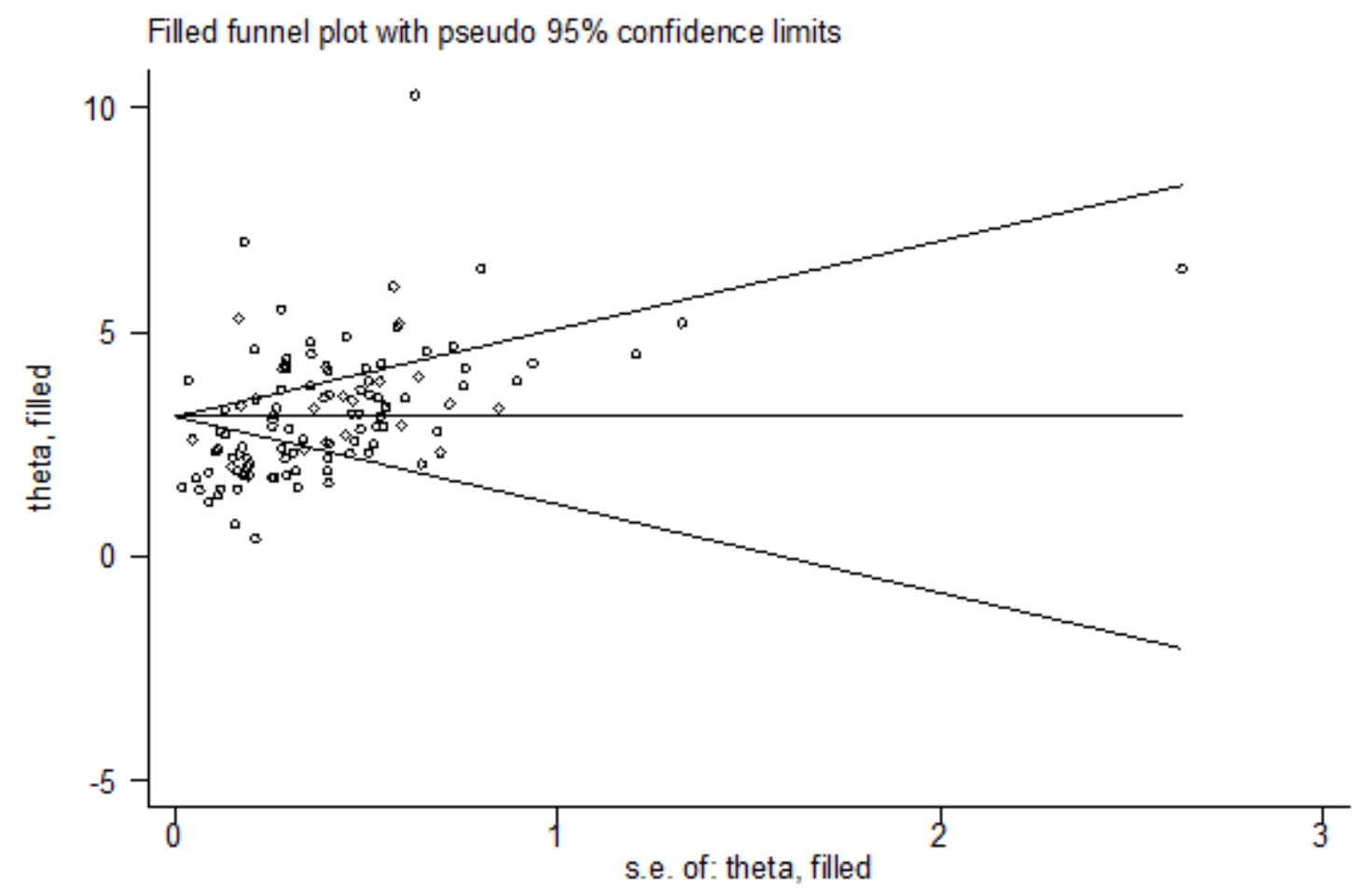

b. S1 amplitudes (Bipolar disorder)

Filled funnel plot with pseudo $95 \%$ confidence limits

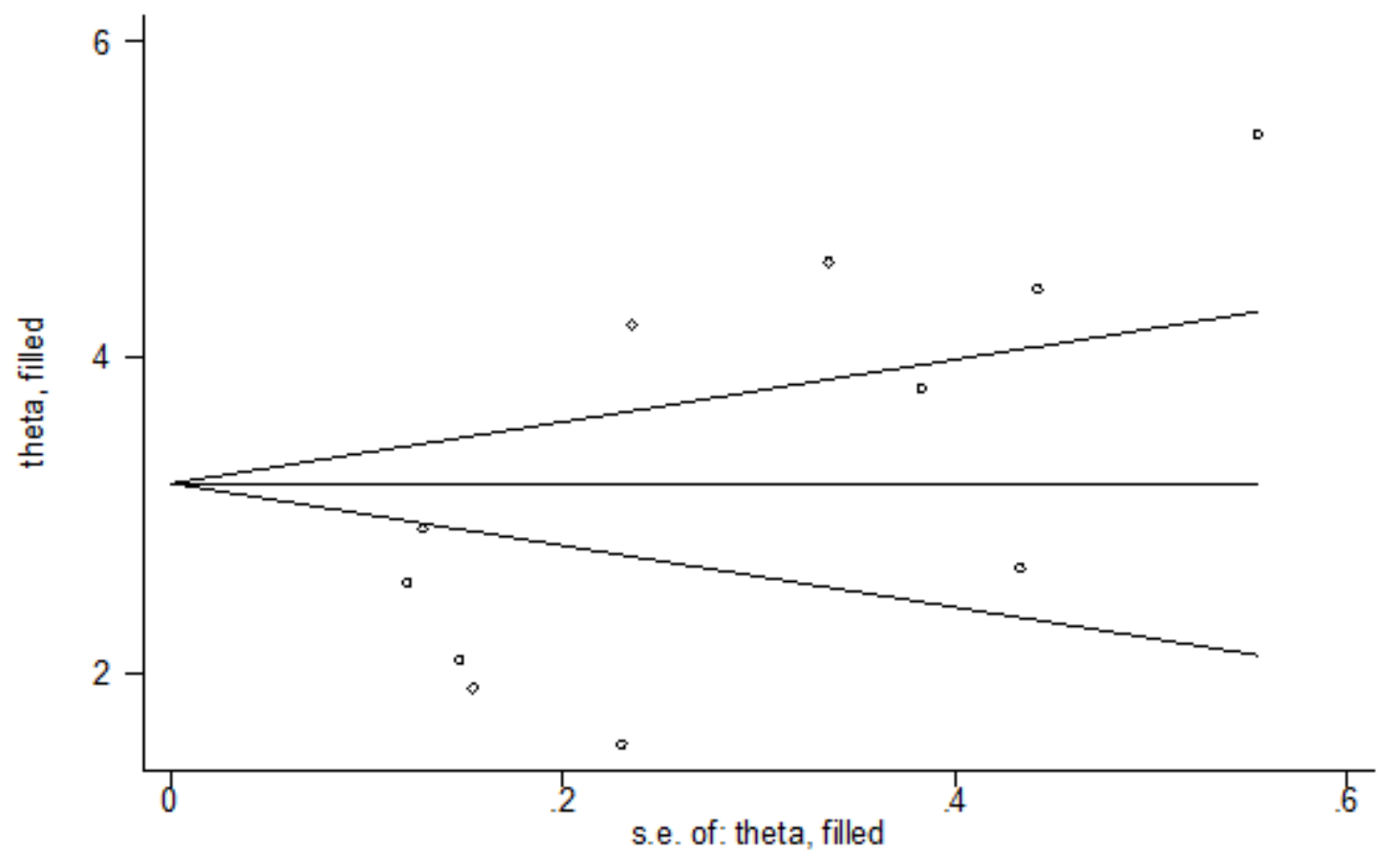


c. S1 amplitudes (Healthy controls)

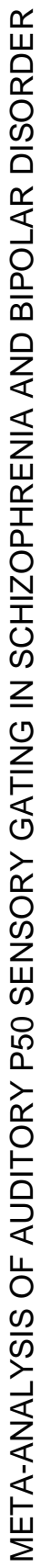

Filled funnel plot with pseudo $95 \%$ confidence limits

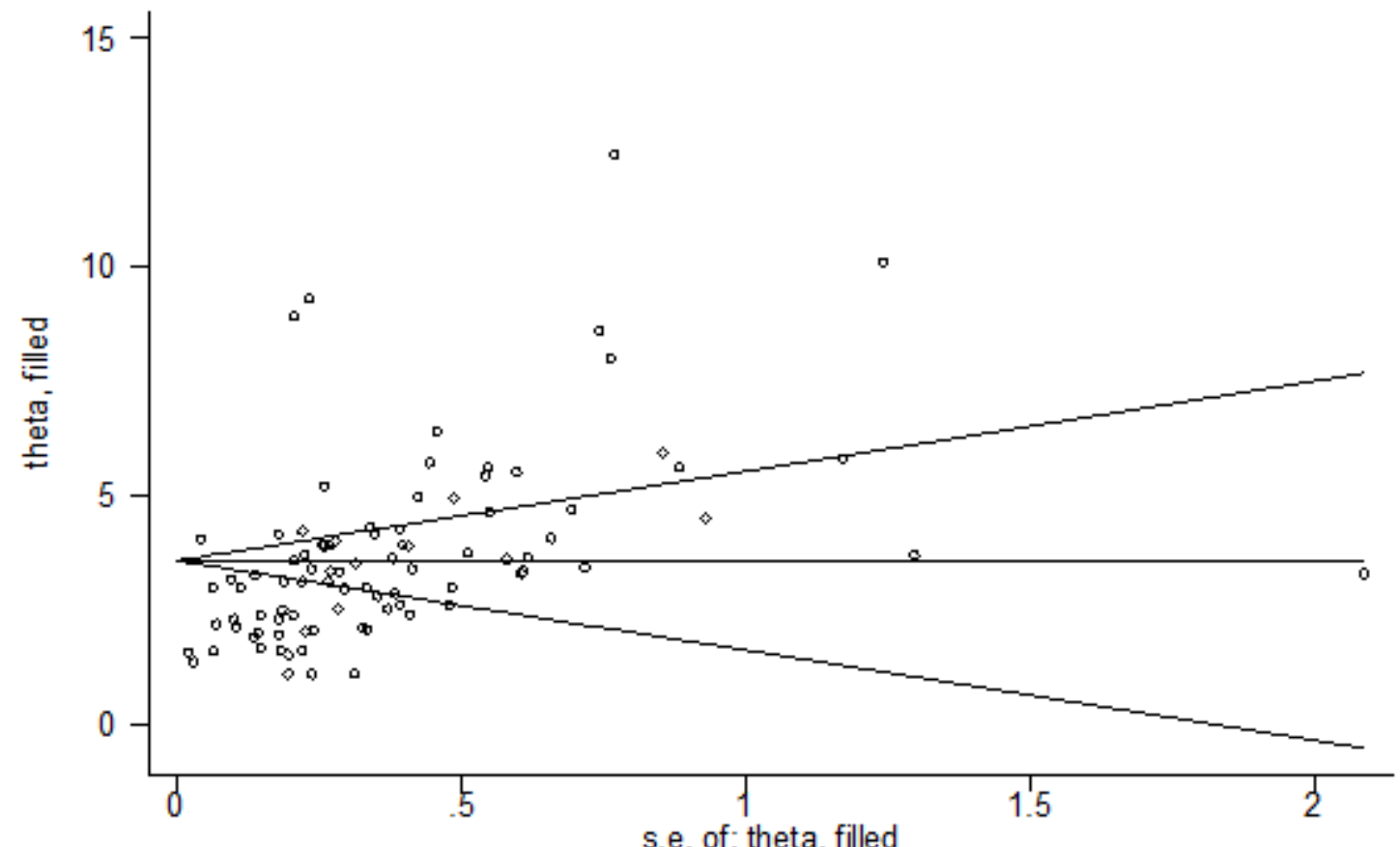

d. S1 amplitudes (First-degree relatives)

Filled funnel plot with pseudo $95 \%$ confidence limits

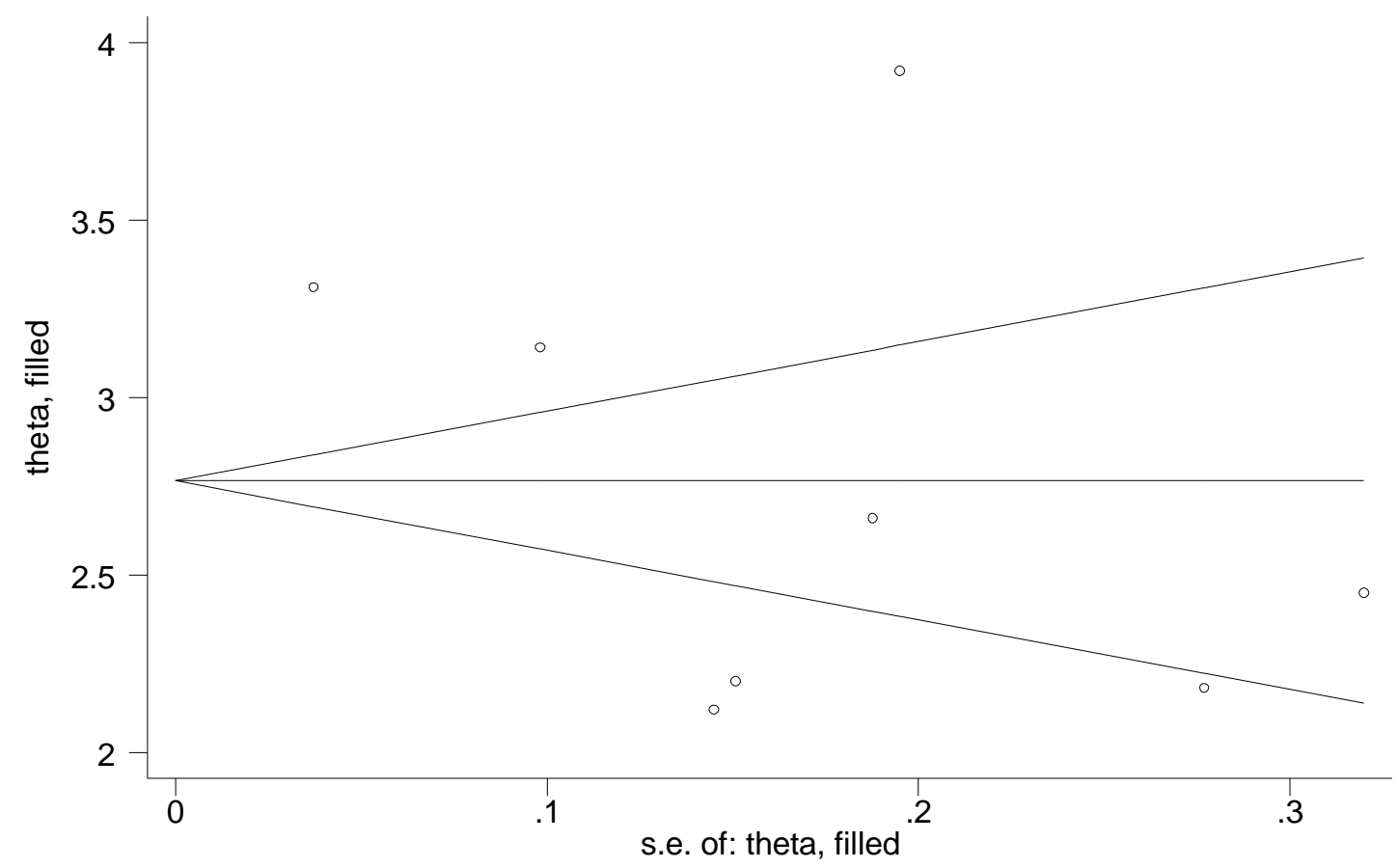


e. S2 amplitudes (Schizophrenia)

Filled funnel plot with pseudo $95 \%$ confidence limits

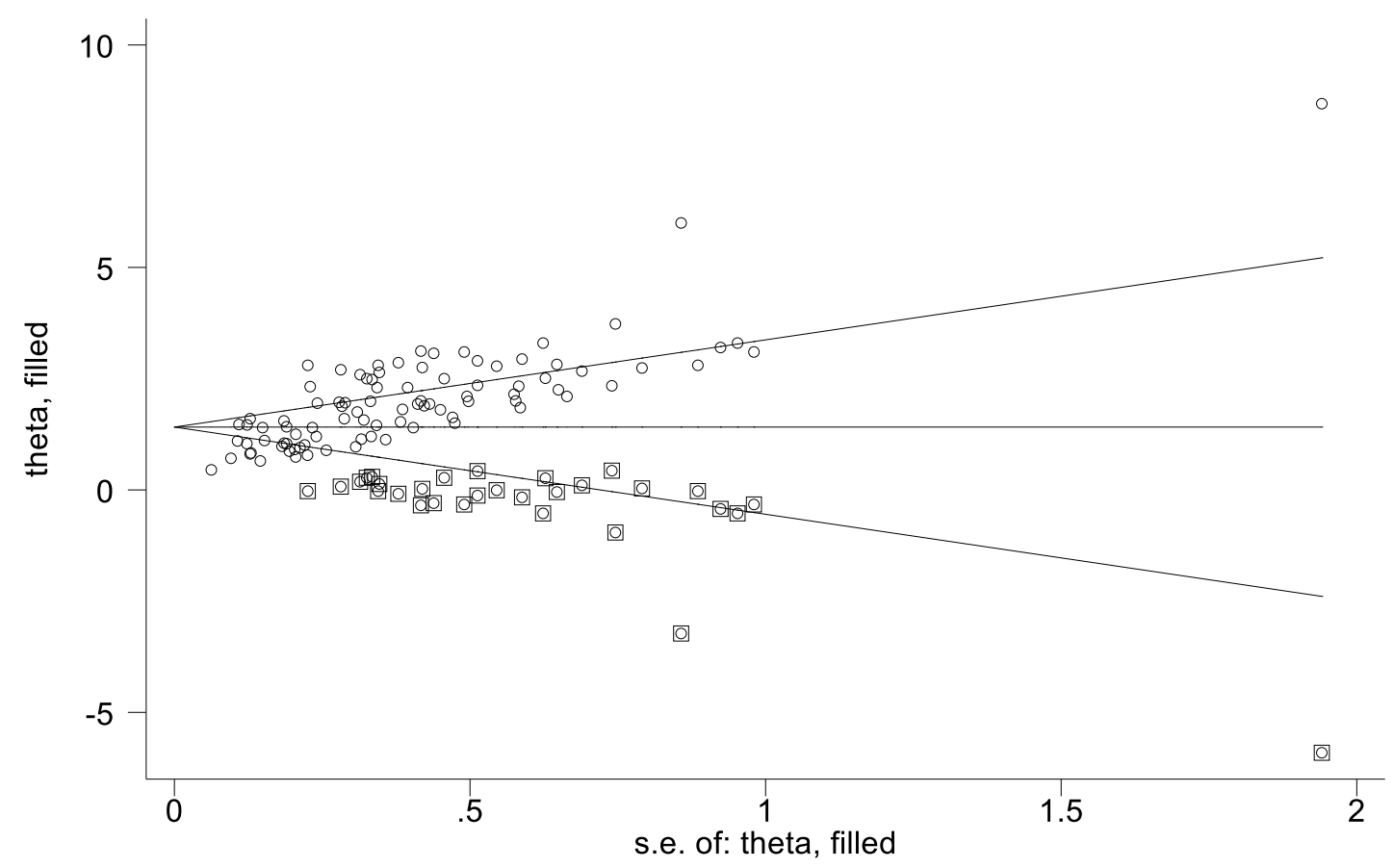

f. S2 amplitudes (Bipolar disorder)

Filled funnel plot with pseudo $95 \%$ confidence limits

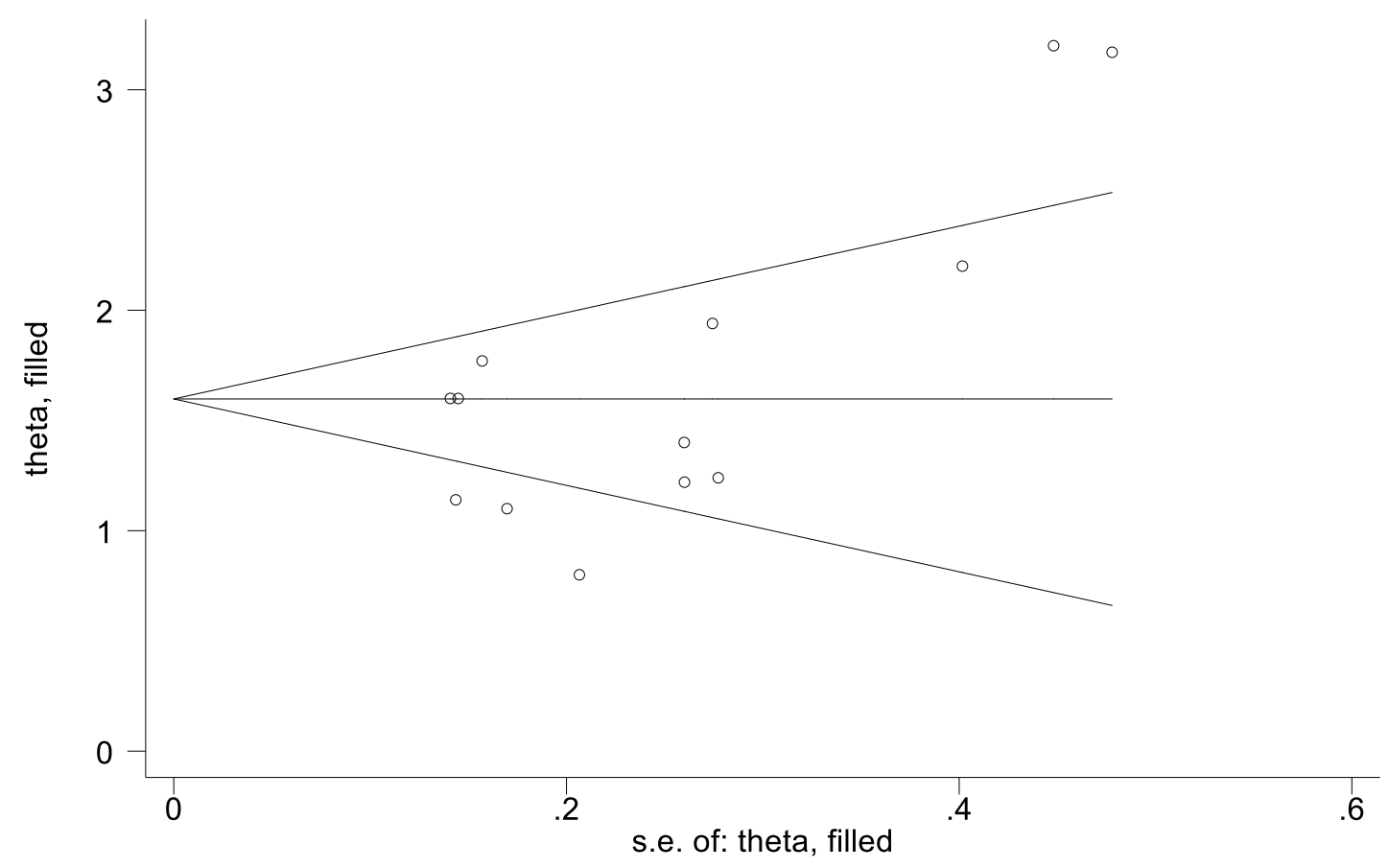


g. S2 amplitudes (Healthy controls)

Filled funnel plot with pseudo $95 \%$ confidence limits

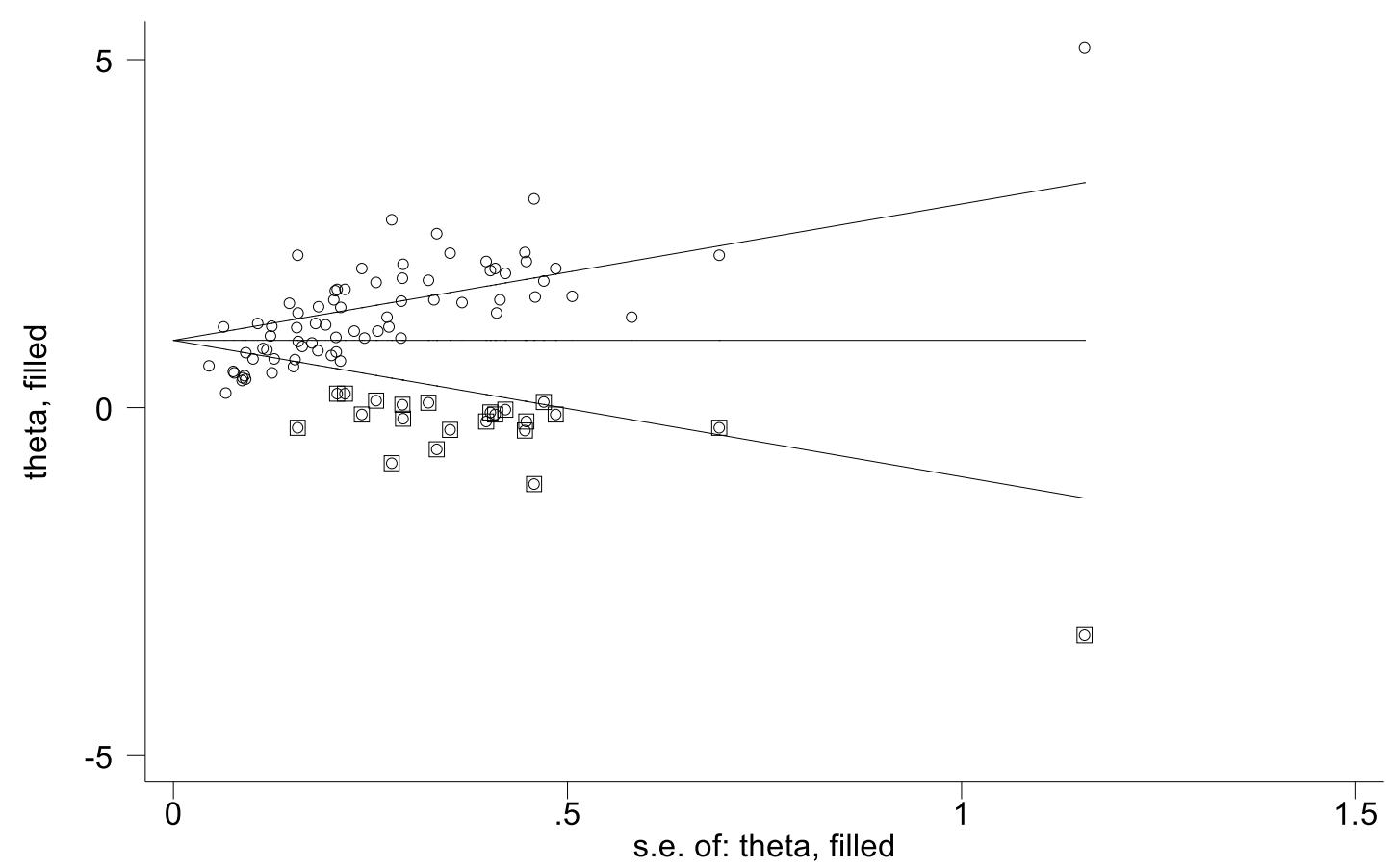

h. S2 amplitudes (First-degree relatives)

Filled funnel plot with pseudo $95 \%$ confidence limits

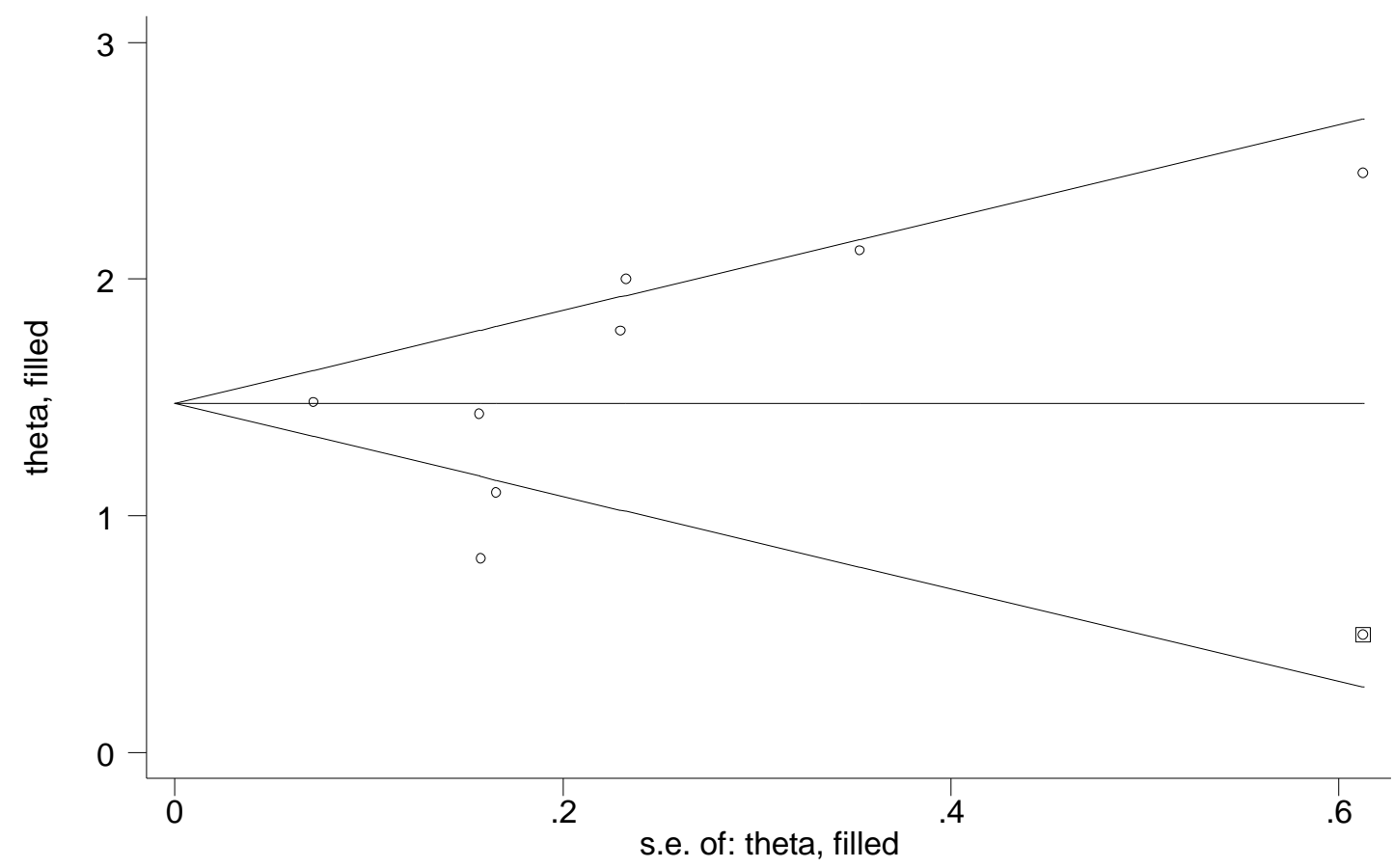


i. S2/S1 ratio (Schizophrenia)

Filled funnel plot with pseudo $95 \%$ confidence limits

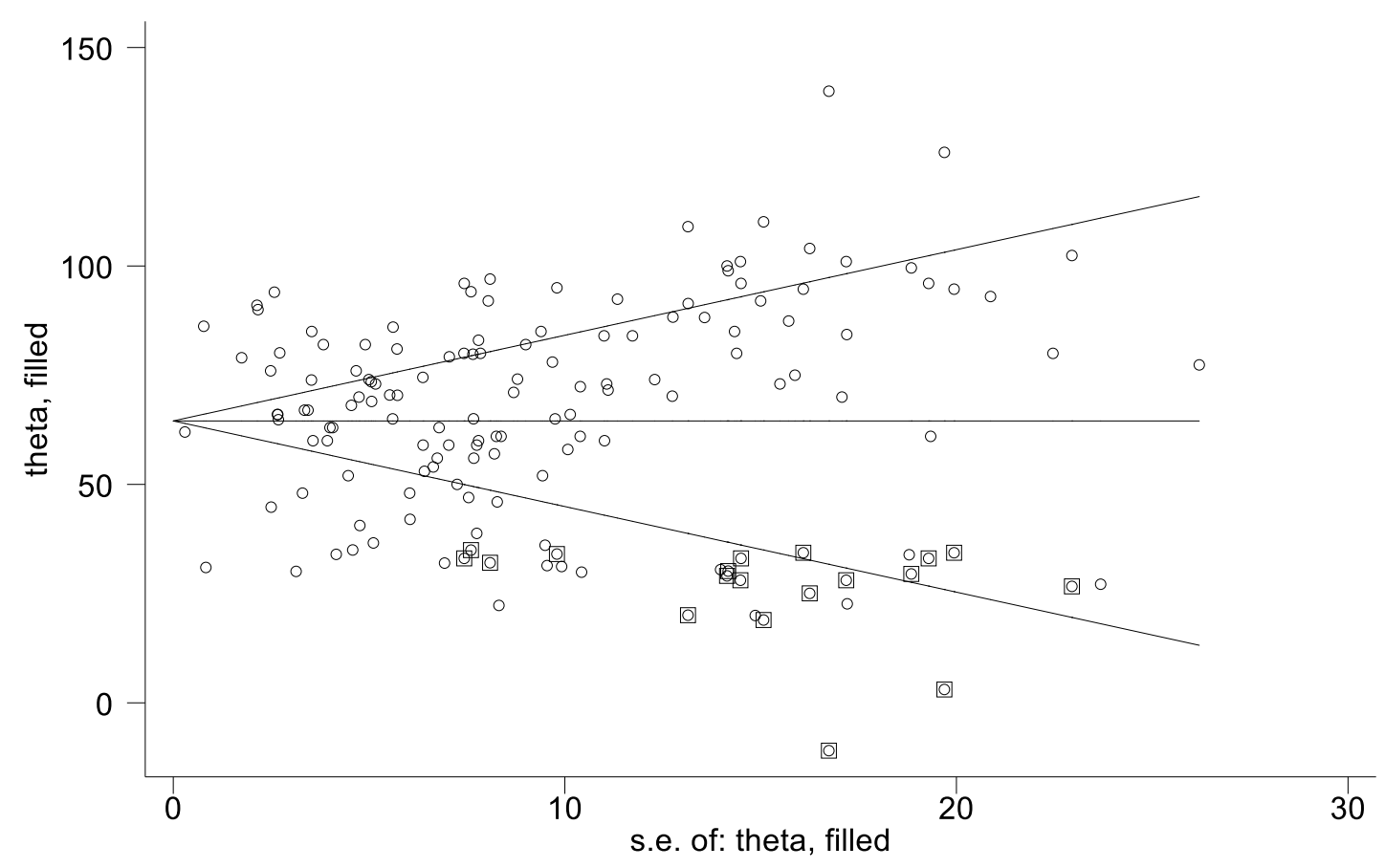

j. S2/S1 ratio (Bipolar disorder)

Filled funnel plot with pseudo $95 \%$ confidence limits

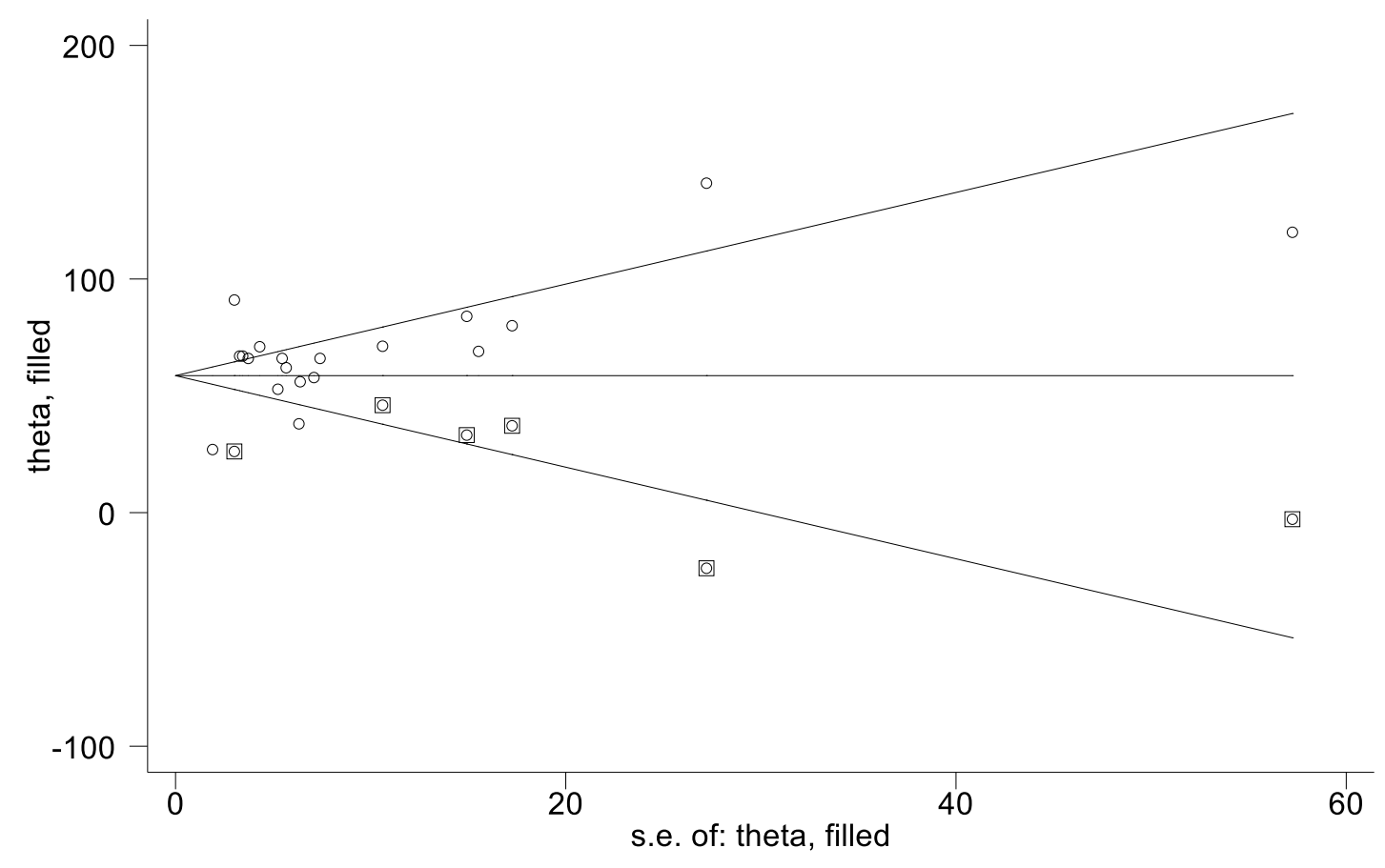


k. S2/S1 ratio (Healthy controls)

Filled funnel plot with pseudo $95 \%$ confidence limits

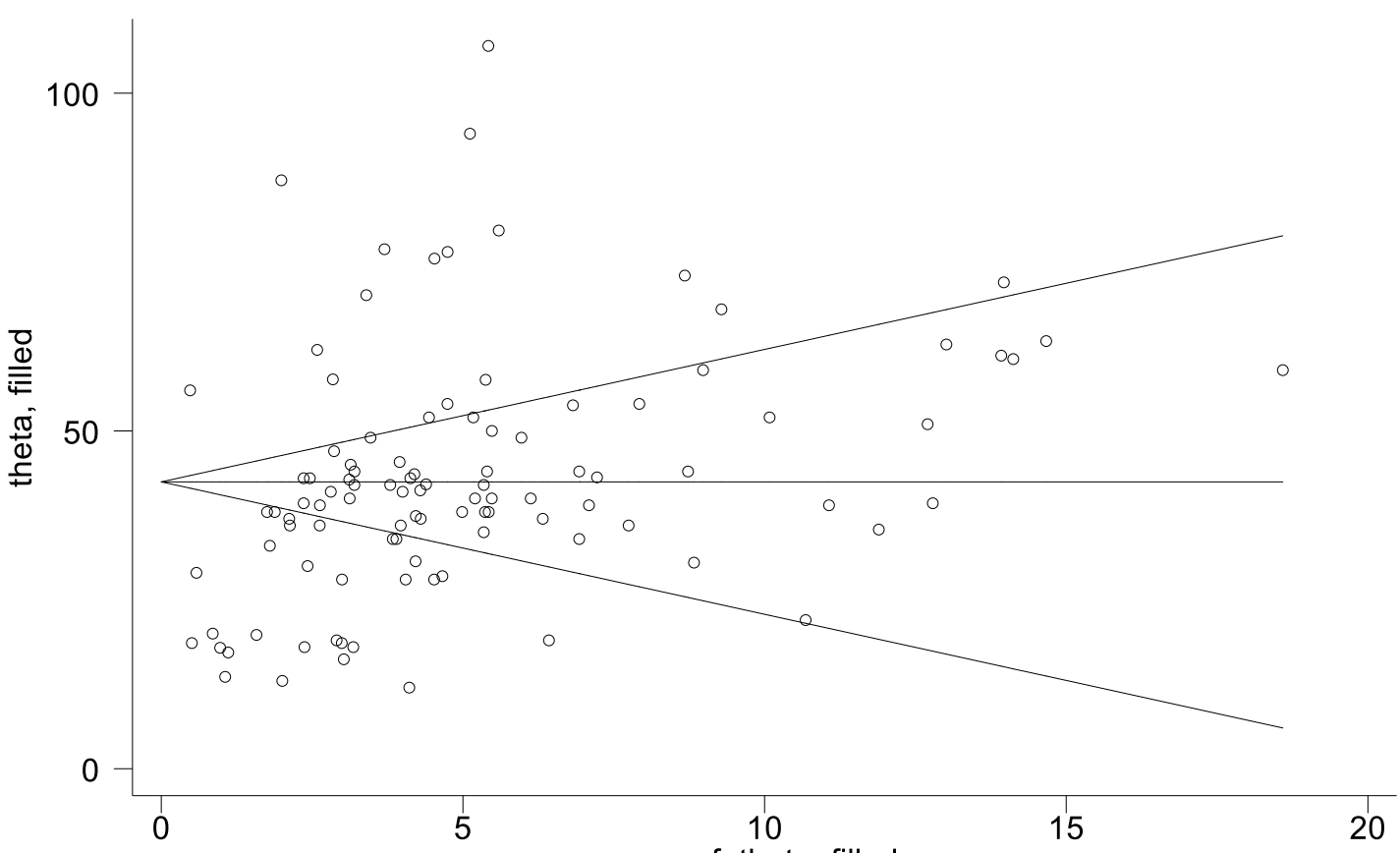

s.e. of: theta, filled

1. S2/S1 ratio (First-degree relatives)

Filled funnel plot with pseudo $95 \%$ confidence limits

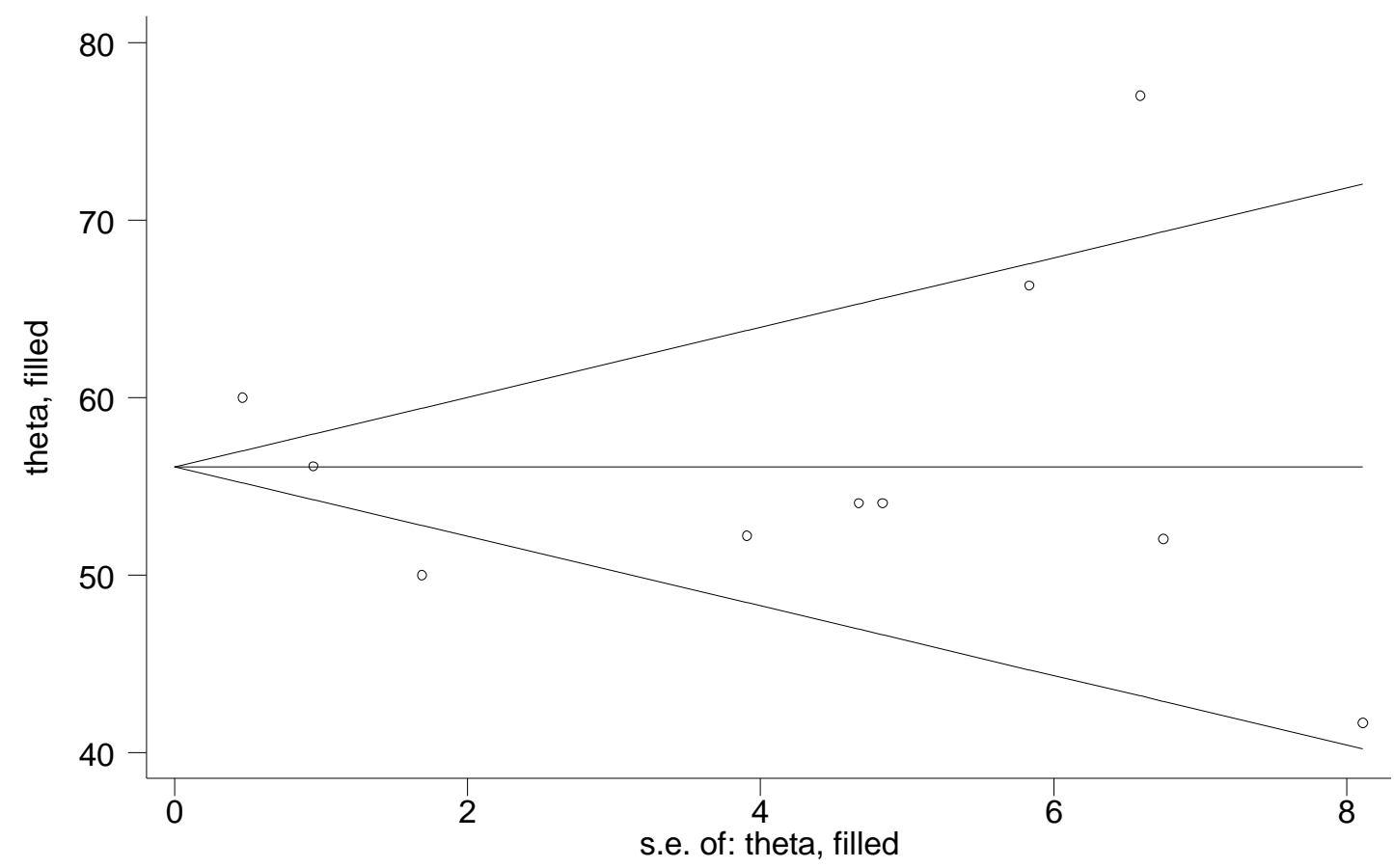


m. S1- S2 difference (Schizophrenia)

Filled funnel plot with pseudo $95 \%$ confidence limits

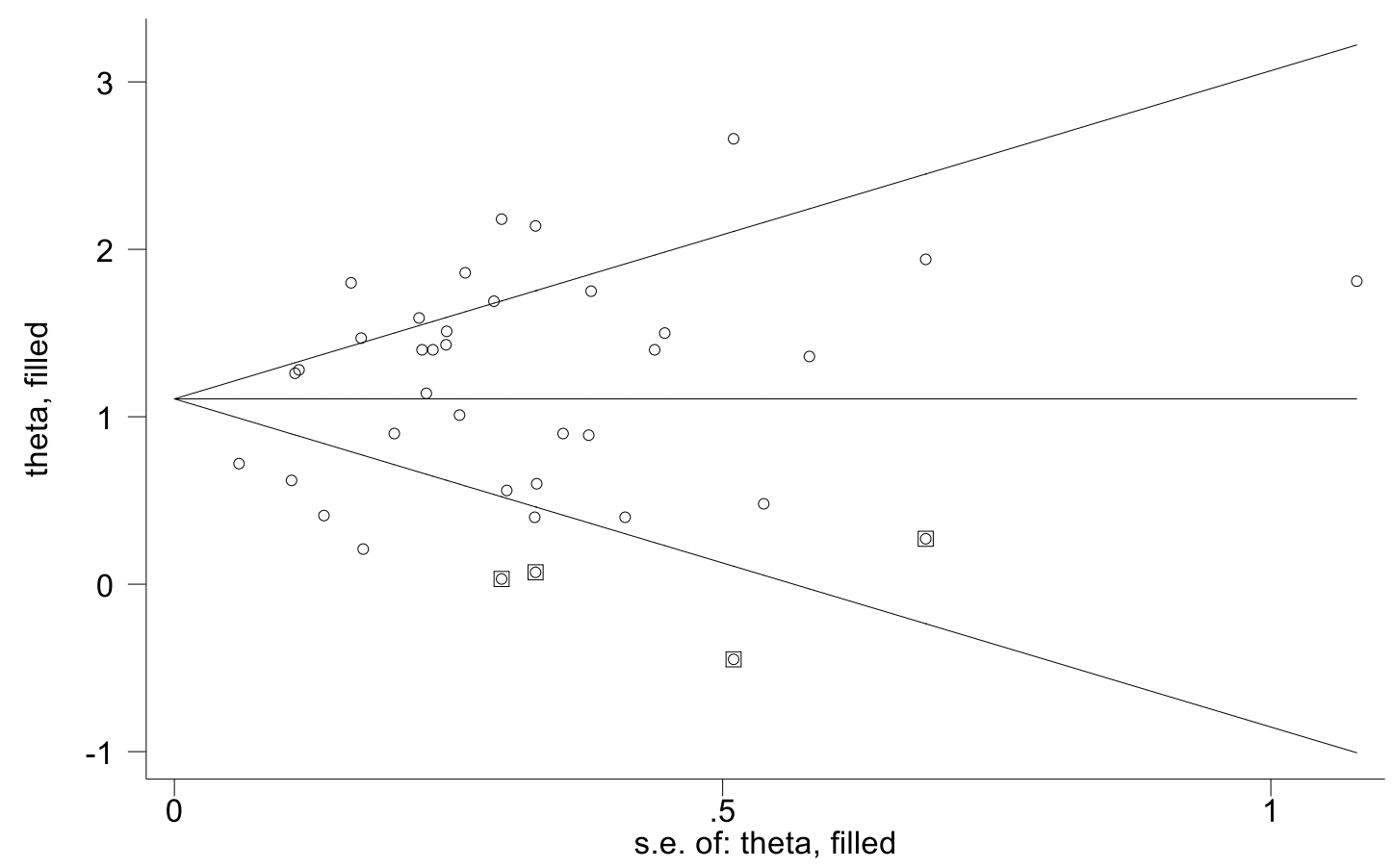

n. S1-S2 difference (Bipolar disorder)

Filled funnel plot with pseudo $95 \%$ confidence limits

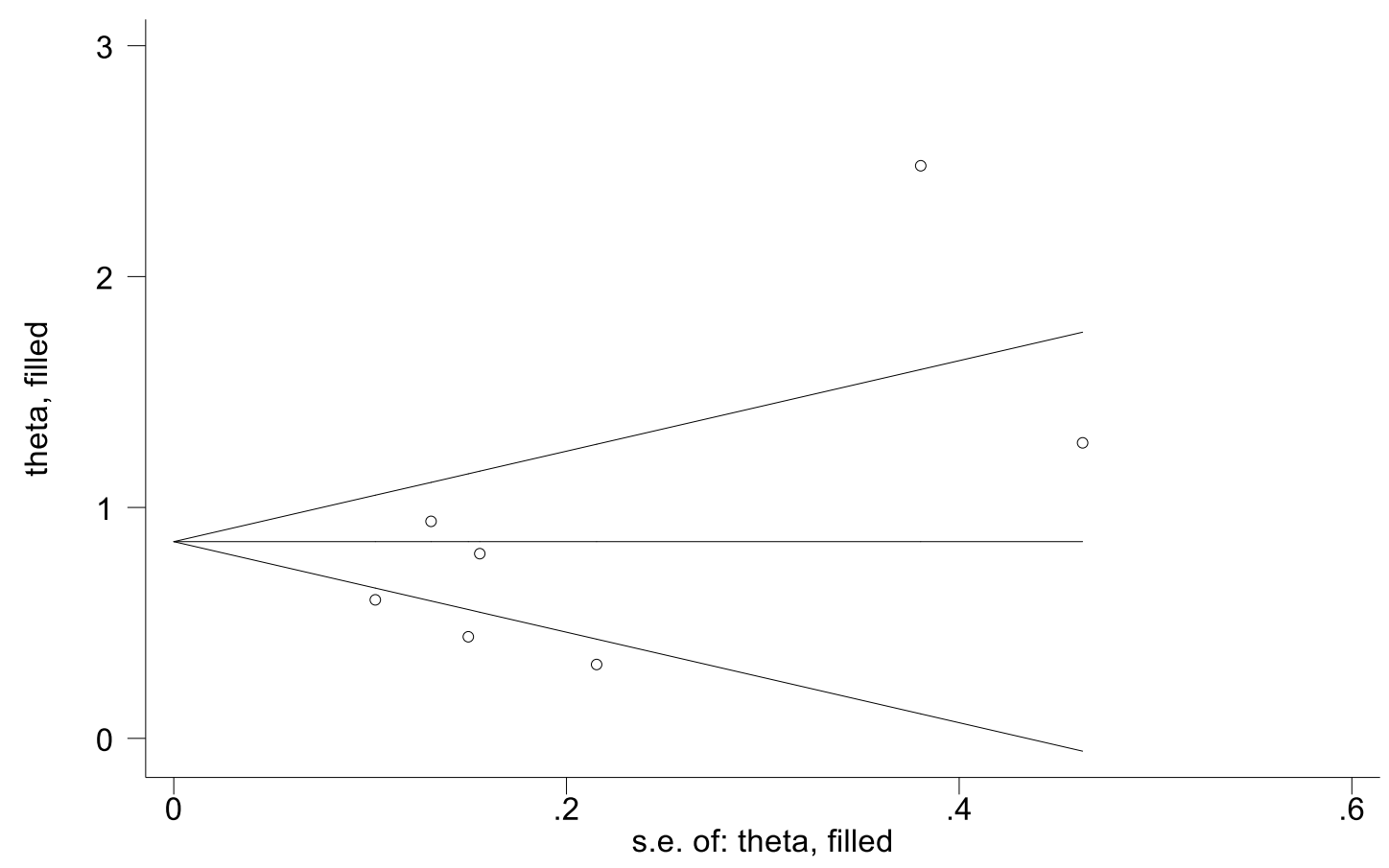


o. S1-S2 difference (Healthy controls)

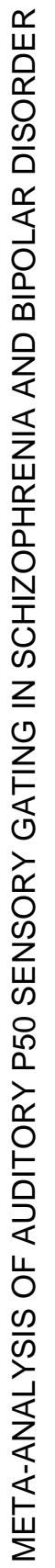

Filled funnel plot with pseudo $95 \%$ confidence limits

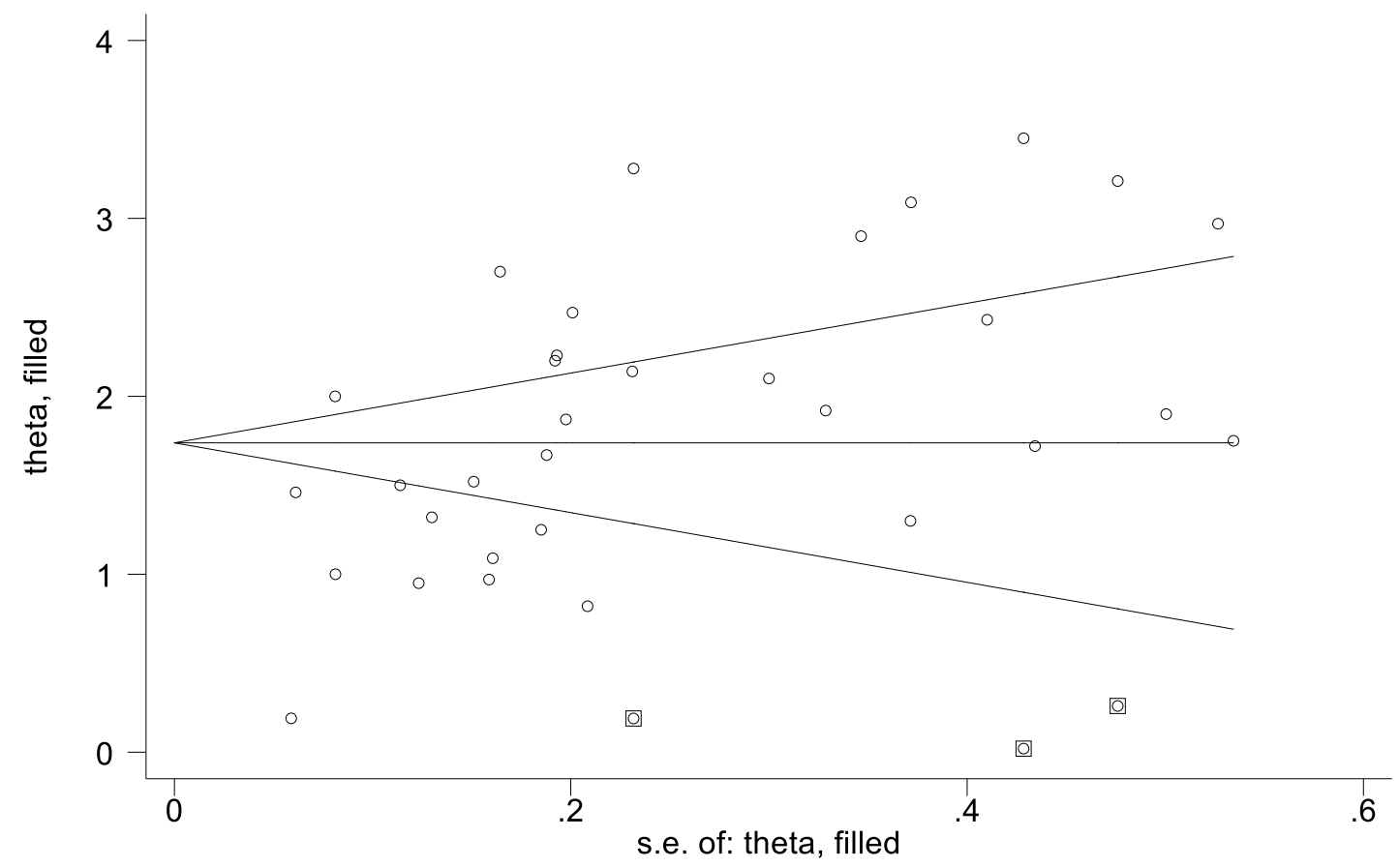

p. S1 - S2 difference (First-degree relatives)

Filled funnel plot with pseudo 95\% confidence limits

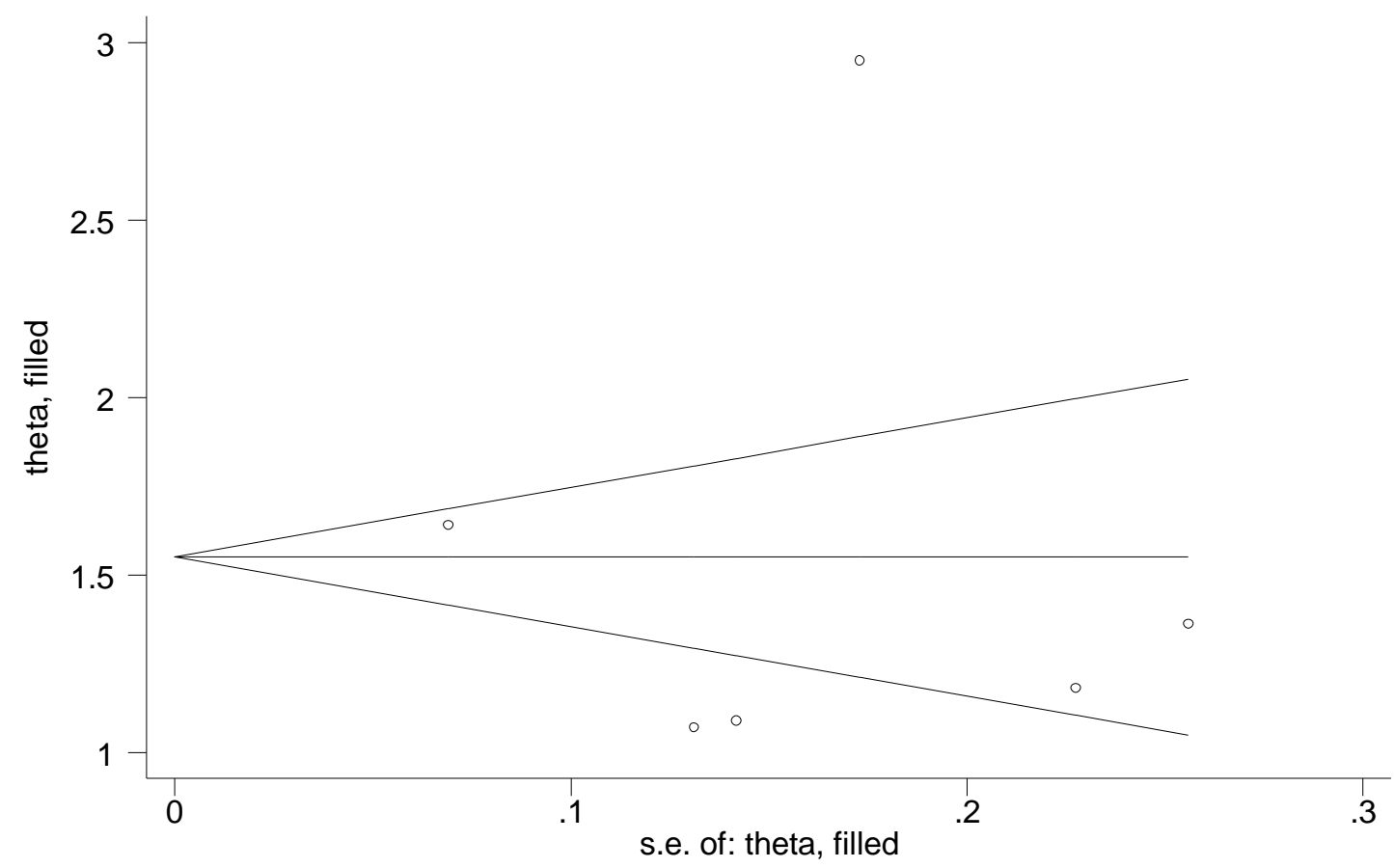




\section{CHAPTER 6}

Investigation of Heschl's gyrus and planum temporale in patients with schizophrenia and bipolar disorder: A proton magnetic resonance spectroscopy study

M.I. Atagün, E.M. Şıkoğlu, S.S. Can, G. Karakaş-Uğurlu, S. Ulusoy-Kaymak, A. Çayköylü, O. Algın, M.L. Phillips, C.M. Moore, D. Öngür

Schizophrenia Research 2015; 161: 202-209 


\section{Abstract}

Background: Superior temporal cortices include brain regions dedicated to auditory processing and several lines of evidence suggest structural and functional abnormalities in both schizophrenia and bipolar disorder within this brain region. However, possible glutamatergic dysfunction within this region has not been investigated in adult patients.

Methods: Thirty patients with schizophrenia ( $38.67 \pm 12.46$ years of age), 28 euthymic patients with bipolar I disorder (35.32 \pm 9.12 years of age), and 30 age-, gender- and educationmatched healthy controls were enrolled. Proton magnetic resonance spectroscopy data were acquired using a $3.0 \mathrm{~T}$ Siemens MAGNETOM TIMTrioMR system and single voxel Point REsolved Spectroscopy Sequence (PRESS) in order to quantify brain metabolites within the left and right Heschl's gyrus and planum temporale of superior temporal cortices.

Results: There were significant abnormalities in glutamate $(\mathrm{Glu})(\mathrm{F}(2,78)=8.52, \mathrm{p}<0.0001)$, $\mathrm{N}$-acetyl aspartate $(\mathrm{tNAA})(\mathrm{F}(2,81)=5.73, \mathrm{p}=0.005)$, creatine $(\mathrm{tCr})(\mathrm{F}(2,83)=5.91, \mathrm{p}=0.004)$ and inositol $(\mathrm{Ins})(\mathrm{F}(2,82)=8.49, \mathrm{p}<0.0001)$ concentrations in the left superior temporal cortex. In general, metabolite levels were lower for bipolar disorder patients when compared to healthy participants. Moreover, patients with bipolar disorder exhibited significantly lower $\mathrm{tCr}$ and Ins concentrations when compared to schizophrenia patients. In addition, we have found significant correlations between the superior temporal cortex metabolites and clinical measures.

Conclusion: As the left auditory cortices are associated with language and speech, left hemisphere specific abnormalities may have clinical significance. Our findings are suggestive of shared glutamatergic abnormalities in schizophrenia and bipolar disorder.

Keywords: Schizophrenia, Bipolar disorder, Glutamate, Glutamine, Magnetic resonance spectroscopy, Superior temporal cortex 


\section{Introduction}

Emil Kraepelin's dichotomous approach to discriminate schizophrenia and bipolar disorder relies on their different clinical courses. However, there has been discrepancy between Kraepelin's approach and studies showing co-aggregation of schizophrenia and bipolar disorder in families as well as shared susceptibility genes (Bramon and Sham, 2001; Craddock and Owen, 2005). The challenge arises due to the presence of overlapping symptoms between schizophrenia and bipolar disorder, but yet the desire for clear diagnostic categorization. Dimensional concepts suggest focusing on symptom domains rather than diagnostic categories (Cuthbert, 2014; Keshavan and Ongur, 2014). These symptom domains in conjunction with biological findings may provide insight for shared and distinct mechanisms underlying the disorders. Superior temporal cortices host primary, secondary and association auditory cortices and have been implicated in the pathophysiology of schizophrenia. Both postmortem and in vivo measurements have shown reductions in volume, thickness and gray matter content of the superior temporal cortex in schizophrenia (Vita et al., 2012; Modinos et al., 2013). Longitudinal studies report progressive gray matter loss in the superior temporal gyrus and more precisely the Heschl's gyrus and planum temporale with progression to psychosis and development of delusions (Vita et al., 2012).Moreover, left superior temporal cortices have been associated with symptom domains such as auditory hallucinations (Dierks et al., 1999; Jardri et al., 2011; Kuhn and Gallinat, 2012; Modinos et al., 2013; Shinn et al., 2013) and thought disorder (Seese et al., 2011; Shenton et al., 1992) in psychosis. These findings suggest the superior temporal gyrus as a highly relevant location for the neurobiology and development of psychosis (Shenton et al., 1992; Rajarethinam et al., 2000; Takahashi et al., 2006; Seese et al., 2011). On the other hand, two meta-analyses of volumetric studies of superior temporal cortices did not report any significant differences between patients with bipolar disorder and healthy participants (Kempton et al., 2008; Arnone et al., 2009). However, primary and secondary auditory cortices are located in the region and functional studies consistently reported auditory processing disturbances in both schizophrenia (Dierks et al., 1999;Umbricht and Krljes, 2005; Domjan et al., 2012) and bipolar disorder (Hall et al., 2009; Oribe et al., 2010). Since glutamate is the major excitatory neurotransmitter and since the EEG signal consists of excitatory end synaptic potentials, auditory processing deficits detected in both schizophrenia (Umbricht and Krljes, 2005; Oribe et al., 2010) and bipolar disorder (Hall et al., 2009; Ethridge et al., 2012; Atagun et al., 2014) could potentially be due to glutamatergic dysfunction in the auditory cortices (Javitt, 2009). Glutamate-modulating agents have been found to be efficacious in the treatment 
of mood disorders both in pre-clinical (Skolnick et al., 2009) and clinical studies (Sanacora et al., 2008; Machado-Vieira et al., 2012). Current psychotomimetics also modulate different components of the glutamatergic system (for reviews: (Machado-Vieira et al., 2012; Sanacora et al., 2008)). Chronic treatment with lamotrigine, valproate or lithium is likely to effect glutamatergic system through a variety of mechanisms (for reviews: (Colla et al., 2009; Gigante et al., 2012; Schifitto et al., 2009; Soeiro-de-Souza et al., 2013; Yatham et al., 2009)). Therefore, the nature and extent of the glutamatergic system abnormalities in patients with schizophrenia and mood disorders require further clarification. Proton magnetic resonance spectroscopy (1HMRS) is a non-invasive neuroimaging technique that can quantify in vivo neurochemical metabolites, including those related to the glutamatergic system. Glutamatergic neurotransmission is thought to be disturbed in both schizophrenia (Goff and Coyle, 2001; Paz et al., 2008; Javitt, 2009, 2010) and bipolar disorder (Sanacora et al., 2008; Machado-Vieira et al., 2012). Moreover, altered glutamatergic metabolites have been reported both in schizophrenia (Brugger et al., 2011; Marsman et al., 2013; Poels et al., 2014a,b) and bipolar disorder (Yildiz-Yesiloglu and Ankerst, 2006; Moore et al., 2007; Yuksel and Ongur, 2010; Ongur et al., 2011). Frontal, anterior cingulate and hippocampal regions are the most frequently studied brain regions and the findings were consistent across these brain regions. Only one study has investigated brain metabolites within the superior temporal gyrus (Seese et al., 2011) and reported a correlation between $\mathrm{N}$-acetyl aspartate (NAA) concentrations and thought disorder in children with schizophrenia. However, to our knowledge, no single 1H MRS study has investigated the superior temporal region and compared the findings in adult patients with schizophrenia and patients with bipolar disorder. Hence, in this study, we utilized 1H MRS to examine neurometabolic changes within superior temporal cortices of adults with schizophrenia or bipolar disorder. Studies by different research modalities consistently report structural and functional abnormalities of auditory cortices, thus we expected to see abnormalities within the neurochemical profile of this region in schizophrenia and/or bipolar disorder.

\section{Methods}

\subsection{Participants and study procedures}

Thirty-five stable patients with paranoid schizophrenia (male or female; between ages of 1859 years old) and 37 euthymic patients with bipolar disorder (male or female; between ages of 20-54 years old) were recruited for the study from the outpatient unit of Ankara Ataturk Training and Education Hospital, Ankara, Turkey. Five of the schizophrenia and 3 of the bipolar 
patients could not attend the MRI session and hence were excluded. Moreover, 5 of the bipolar patients had bipolar disorder II and therefore were not included in the analysis and we could not acquire neuroimaging data from 1 of the bipolar patients due to technical issues. In addition, 30 age-, gender-, and education matched healthy participants (male or female; between ages of 18-58 years old) were enrolled in the study. All participants provided written consent upon receiving detailed information about the study. The Ethical Committee of Yildırım Beyazit University approved the study.

All participants underwent a clinical interview administered by a psychiatrist (MIA) and diagnoses were checked with the Structured Clinical Interview according to the DSM-IV (SCID-I) (First et al., 1996). The clinical interview assessed the participants' demographics and medical/psychiatric history. Patients with bipolar disorder were evaluated using the Young Mania Rating Scale (YMRS) (Young et al., 1978) and the Hamilton Depression Rating Scale (HDRS) (Hamilton, 1960). Schizophrenia patients were evaluated with Scale for the Assessment of Positive Symptoms (SAPS) (including subscores for delusion and hallucination) (Andreasen, 1984) and Scale for the Assessment of Negative Symptoms (SANS) (Andreasen, 1983). All patients were also evaluated with the Brief Psychiatric Rating Scale (BPRS) (Overall and Gorham, 1962) and the Edinburgh Handedness Inventory (EHI) (Oldfield, 1971). All participants were free of any brain damage/surgeries or metabolic systemic diseases such as diabetes mellitus or hypertension or current or lifetime psychiatric comorbidity or substance abuse. Psychotropic medications except for benzodiazepines were allowed. Exclusion criteria also included any contraindications to MRI scan. In addition deafness or heading aids were excluded to avoid any possible plasticity effects as a result of hearing disabilities, as we are particularly interested in the temporal lobe. Finally, all participants completed an MRI scanning session.

\subsection{Neurolmaging data acquisition}

Data were acquired on a $3.0 \mathrm{~T}$ Siemens MAGNETOMTIM Trio whole body MR system (Siemens, Erlangen, Germany) with a thirty two channel phased-array head coil at the UMRAM National Magnetic Resonance Research Center, Ankara, Turkey. T1-weighted anatomical. MRI (MPRAGE sequence, $256 \AA ̊ 256$ voxels, TR: 2000 ms, TE: 3.02 ms, FOV read: 215, FOV phase: 100, slice thickness: 0.84, 192 slices) were collected for diagnostic and localization purposes. Voxels $(20 \mathrm{~mm} \AA \sim 20 \mathrm{~mm} \AA \sim 20 \mathrm{~mm})$ were placed consecutively in the left and right Heschl's gyrus and planum temporale regions (Fig. 1) and proton magnetic resonance 
spectroscopy (1H MRS) data were acquired using the single voxel Point REsolved Spectroscopy Sequence (PRESS) (TE=30 ms, TR=2000 ms, 128 averages) in order to quantify brain metabolites (Fig. 1). Manually adjusted B0 shimming was applied around the voxel as implemented on our MR system.

\subsection{Neurolmaging data analysis}

The proton spectrawere fit using LCModel (Version6.3.0) toquantify the metabolite concentrations. LCModel analyzes in vivo proton spectra as a linear combination ofmodel in vitro spectra from individualmetabolite solutions (Provencher, 1993, 2001) by utilizing builtin (simulated) radial basis sets. The basis set used for this study included alanine, aspartate, creatine $(\mathrm{Cr})$, phosphocreatine (PCr), $\gamma$ aminobutyric acid (GABA), glucose, glutamate (Glu), glutamine (Gln), glycerophosphocholine (GPC), phosphocholine (PCh), glutathione (GSH), inositol (Ins), lactate, N-acetyl aspartate (NAA), N-acetyl aspartyl glutamate (NAAG), scylloinositol, taurine as well as macromolecules and lipids. Total NAA (tNAA, NAA and NAAG), Glu, Glx (Glu and Gln), total creatine (tCr, $\mathrm{Cr}$ and $\mathrm{PCr}$ ), total choline (tCh, GPC and PCh), and Ins were quantified and fits with Cramer-Rao lower bounds (CRLB, estimated error of the metabolite quantification) of greater than $10 \%$ were classified as not reliably detected and excluded from further analysis. The structural T1-weighted images were segmented using SPM8 (Statistical Parameter Mapping-Welcome Department of ImagingNeuroscience, London, UK; (http://www.fil.ion.ucl.ac.uk/spm/software/spm8/)) to determine the gray matter, white matter and CSF contributions to the voxel of interest.

\subsection{Statistics}

Statistical analysis was performed using SPSS 21 (Armonk, NY: IBM Corp.). First, we conducted an outlier analysis for each metabolite within each group of participants. The values that were two standard deviations away from the mean of their corresponding groups were eliminated from further analysis. The remaining data were subjected to univariate ANOVA to compare the findings across 3 groups, i.e. schizophrenia patients vs. patients with bipolar disorder vs. healthy participants; and metabolite levels were the dependent factors for each comparison. Since we have 6 different metabolites (i.e. tNAA, Glu, Glx, tCr, tCho and Ins), to account for the multiple comparisons we considered p b 0.008 to be significant. We also performed post-hoc analyses with Bonferonni corrections and for this part of the analysis we considered $\mathrm{p} \mathrm{b} 0.05$ to be significant. In addition, we conducted an ANCOVA to investigate the 
potential covariates that have clinical importance and cover a range of values within each group (i.e. age of onset and duration of disorder as well as medication effects). Further, we conducted a Pearson's correlation analysis to explore the relationship between the brain metabolites and the clinical measures and for these analyses we considered $\mathrm{p}<0.05$ to be significant.

Figure 1. Representative proton spectrum and LCModel fit (red) with sample placement of voxels (yellow) in the left and right superior temporal gyri.

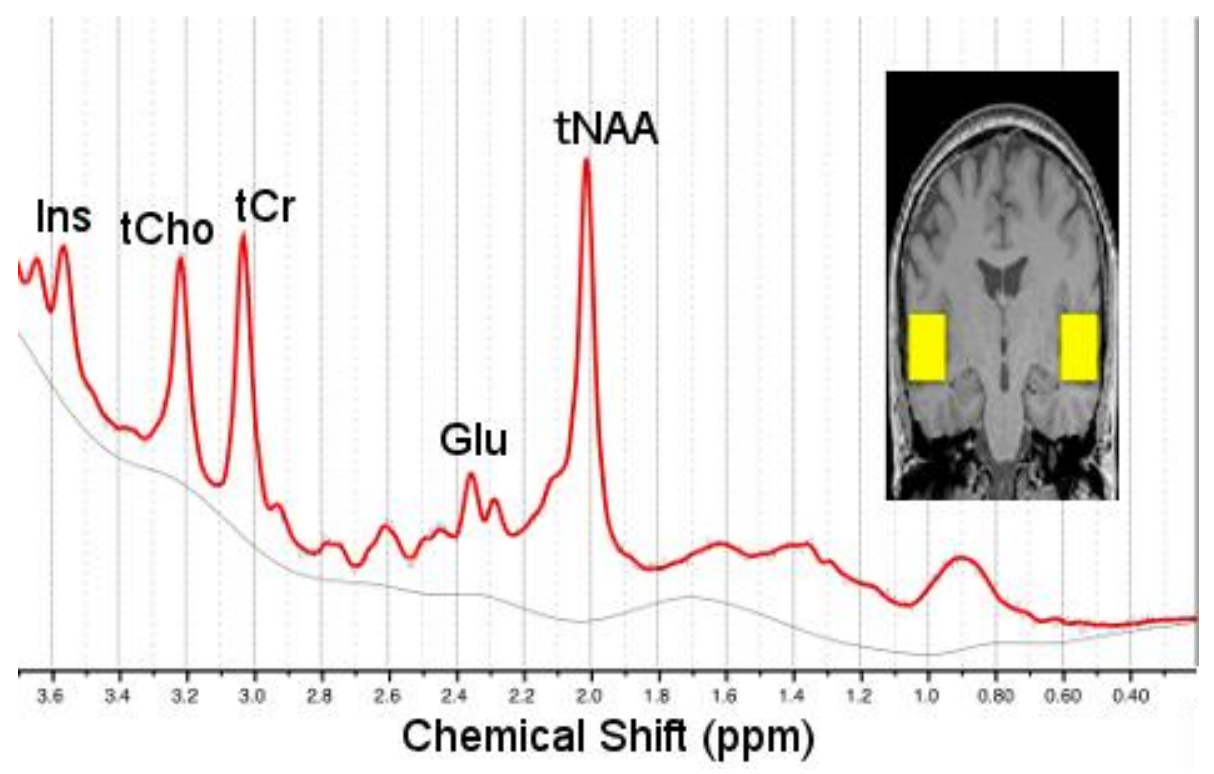

\section{Results}

\subsection{Participant demographics}

Thirty remitted patients with paranoid schizophrenia (18 male; average age: $38.7 \pm 12.5$ years) and 28 euthymic patients with bipolar disorder (13 male; average age: $35.3 \pm 9.1$ years) and 30 healthy participants (13 male; average age: $32.8 \pm 10.7$ years) were included in the study. There were no significant differences in any of the demographic variables across the groups $(p<0.05)$. Age of onset varied between the ages of 10 and 38 years for schizophrenia patients (average age of onset: $22.5 \pm 6.4$ years), and ages of 12 and 48 years (average age of onset: $23.6 \pm 8.7$ years) for patients with bipolar disorder. Schizophrenia patients scored significantly higher than patients with bipolar disorder in BPRS $(\mathrm{F}(1,56)=29.45, \mathrm{p}<0.0001)$. The demographic and clinical characteristics of the sample are listed in Table 1. In the schizophrenia group, there were three medication-free patients, 26 patients were on various mono-therapy antipsychotic 
medications and only 1 patient was taking both antipsychotic and valproate together. In the bipolar disorder group, there were five medication-free patients; the rest were on medication (valproate mono-therapy $(n=3)$, combination therapy of valproate and antipsychotics $(n=3)$, lithium monotherapy $(n=6)$, combination therapy of lithium and antipsychotics $(n=3)$, combination of valproate, lithium and antipsychotics $(n=1)$, antipsychotic mono-therapy $(n=7))$.

Table 1. Clinical characteristics of participants.

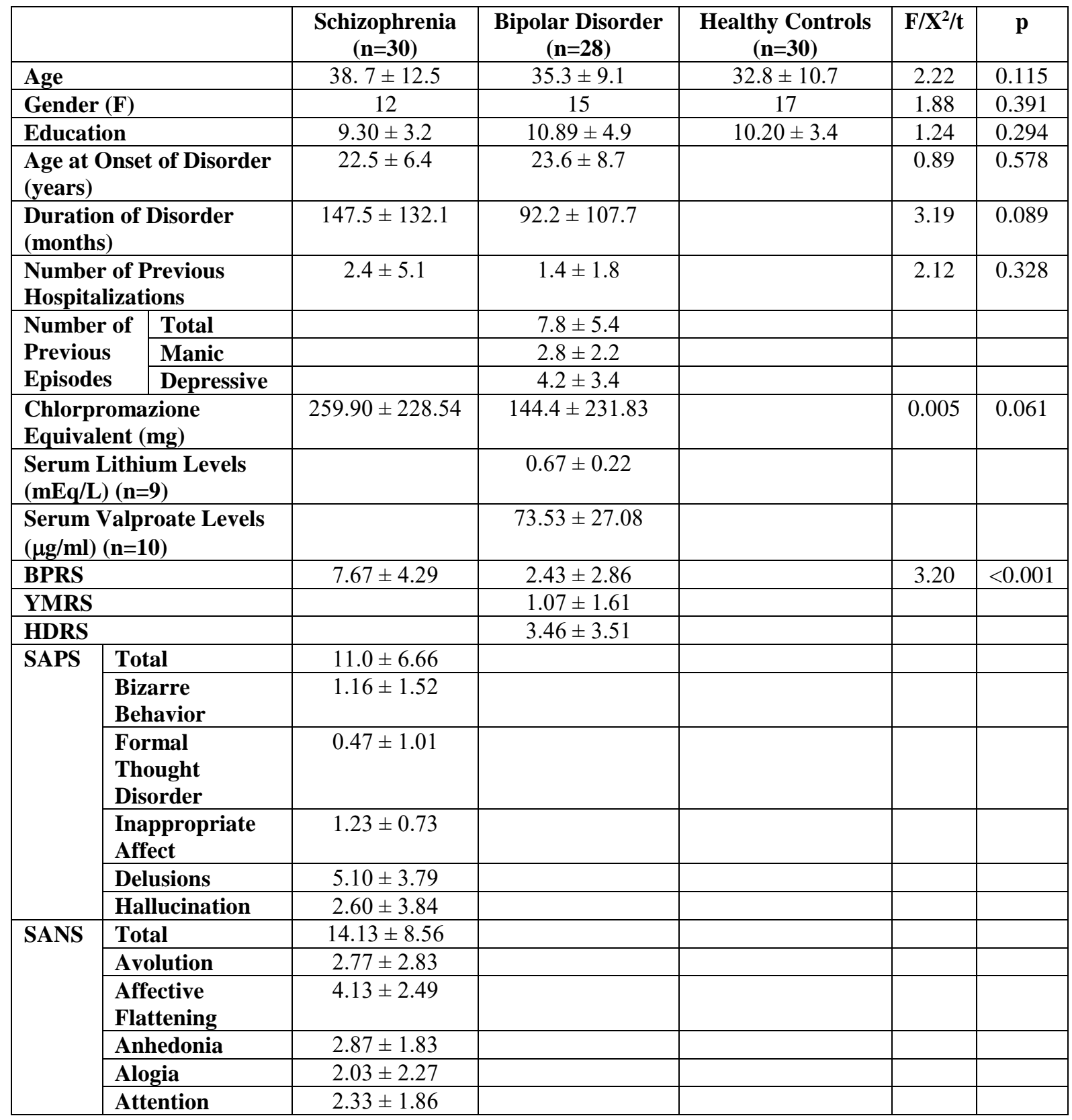

Averages are reported with standard deviations. One-way ANOVA, Chi-Square test and t test. BPRS: Brief Psychiatric Rating Scale, HDRS: Hamilton Depression Rating Scale, SANS: Schedule for the Assessment of Negative Symptoms, SAPS: Schedule for the Assessment of Positive Symptoms, YMRS: Young Mania Rating Scale. 


\subsection{Brain metabolites}

We evaluated the brain metabolites from the left and right Heschl's gyrus and planum temporale regions separately. Statistically significant findings are summarized in Table 2. Within the left hemisphere voxel, Glu $(\mathrm{F}(2,78)=8.52$, p<0.0001) (Fig. 2A), tNAA $(\mathrm{F}(2,81)=5.73, \mathrm{p}=0.005)$ (Fig. 2C), $\operatorname{tCr}(\mathrm{F}(2,83)=5.91, \mathrm{p}=0.004)$ (Fig. 2E) and $\operatorname{Ins}(\mathrm{F}(2,82)=8.49, \mathrm{p}<0.0001)$ (Fig. 2G) levels were statistically significantly different among the three groups. Post-hoc analysis revealed that tNAA, $\mathrm{tCr}$ and Ins levels were similar between schizophrenia patients and healthy

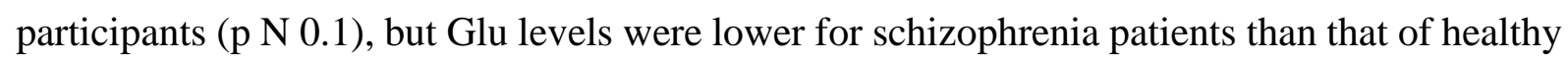
comparisons at a trend level $(\mathrm{p}=0.1)$. For Glu, tNAA, $\mathrm{tCr}$ and Ins, patients with bipolar disorder exhibited lower levels when compared to healthy participants (Glu: $p<0.0001$; tNAA: $p=0.004$; tCr: $\mathrm{p}=0.008$; Ins: $\mathrm{p}=0.003$ ). In addition, $\mathrm{tCr}$ and Ins levels were higher for patients with schizophrenia when compared to patients with bipolar disorder ( $\mathrm{tCr}: \mathrm{p}=0.016$; Ins: $\mathrm{p}=0.001$ ). Due to the wide ranges in age at onset, duration of illness, and medication status, we further investigated the differences in Glu, tNAA, tCr and Ins among patients with bipolar disorder and schizophrenia patients by accounting for age onset, duration of disorder and the chlorpromazine equivalent values of medicated patients; results are summarized in Table 2.

Table 2. ${ }^{1} \mathrm{H}$ MRS findings.

\begin{tabular}{|c|c|c|c|c|c|c|}
\hline & & $\begin{array}{l}\text { Schizophrenia } \\
\qquad(n=30)\end{array}$ & $\begin{array}{c}\text { Bipolar } \\
\text { Disorder } \\
(\mathbf{n}=\mathbf{2 8})\end{array}$ & $\begin{array}{c}\text { Healthy } \\
\text { Controls } \\
(\mathbf{n}=30)\end{array}$ & $\mathbf{F}$ & $\mathbf{p}$ \\
\hline \multicolumn{7}{|l|}{$\begin{array}{c}\text { Left } \\
\text { Hemisphere }\end{array}$} \\
\hline Glu & & $\begin{array}{c}0.00088 \pm \\
0.00011\end{array}$ & $\begin{array}{c}0.00082 \pm \\
0.00010\end{array}$ & $\begin{array}{c}0.00095 \pm \\
0.00013\end{array}$ & $\mathrm{~F}(2,78)=8.52$ & $<0.0001$ \\
\hline \multirow{3}{*}{$\begin{array}{c}\text { Covariates: } \\
\text { (related to } \\
\text { disorder) }\end{array}$} & age onset & & & & $\mathrm{F}(1,50)=4.39$ & 0.041 \\
\hline & duration & & & & $F(1,50)=4.64$ & 0.036 \\
\hline & CPZE & & & & $\mathrm{F}(1,36)=5.78$ & 0.021 \\
\hline tNAA & & $\begin{array}{c}0.00111 \pm \\
0.00016\end{array}$ & $\begin{array}{c}0.00104 \pm \\
0.00015\end{array}$ & $\begin{array}{c}0.00117 \pm \\
0.00013\end{array}$ & $\mathrm{~F}(2,81)=5.73$ & 0.005 \\
\hline \multirow{3}{*}{$\begin{array}{c}\text { Covariates: } \\
\text { (related to } \\
\text { disorder) }\end{array}$} & age onset & & & & $\mathrm{F}(1,52)=3.53$ & 0.066 \\
\hline & duration & & & & $\mathrm{F}(1,52)=3.73$ & 0.059 \\
\hline & CPZE & & & & $\mathrm{F}(1,36)=2.83$ & 0.101 \\
\hline $\mathrm{tCr}$ & & $\begin{array}{c}0.00080 \pm \\
0.00011\end{array}$ & $\begin{array}{c}0.00071 \pm \\
0.00011\end{array}$ & $\begin{array}{c}0.00080 \pm \\
0.00011\end{array}$ & $\mathrm{~F}(2,83)=5.91$ & 0.004 \\
\hline \multirow{3}{*}{$\begin{array}{c}\text { Covariates: } \\
\text { (related to } \\
\text { disorder) }\end{array}$} & age onset & & & & $\mathrm{F}(1,52)=8.91$ & 0.004 \\
\hline & duration & & & & $F(1,52)=7.26$ & 0.009 \\
\hline & CPZE & & & & $\mathrm{F}(1,36)=6.40$ & 0.016 \\
\hline Ins & & $\begin{array}{c}0.00067 \pm \\
0.00010\end{array}$ & $\begin{array}{c}0.00057 \pm \\
0.00012\end{array}$ & $\begin{array}{c}0.00067 \pm \\
0.00011\end{array}$ & $\mathrm{~F}(2,82)=8.49$ & $<0.0001$ \\
\hline \multirow{3}{*}{$\begin{array}{l}\text { Covariates: } \\
\text { (related to } \\
\text { disorder) }\end{array}$} & age onset & & & & $F(1,52)=14.48$ & $<0.0001$ \\
\hline & duration & & & & $F(1,52)=11.25$ & 0.001 \\
\hline & CPZE & & & & $\mathrm{F}(1,35)=6.64$ & 0.014 \\
\hline
\end{tabular}

Absolute quantity averages are reported with standard deviations. Univariate ANOVA.

CPZE: Chlorpromazione Equivalent. 
On the other hand, there were no statistically significant metabolite differences within the right hemisphere voxel (Glu: Fig. 2B; tNAA: Fig. 2D; tCr: Fig. 2F; Ins: Fig. 2H). Since there was no statistically significant differences between groups in terms of voxel tissue content (left hemisphere: gray matter $-(\mathrm{F}(2,84)=0.73, \mathrm{p}=0.48)$, white matter $-(\mathrm{F}(2,84)=0.22, \mathrm{p}=0.81)$, $\operatorname{csf}-\mathrm{F}(2,84)=0.78, \mathrm{p}=0.46$; right hemisphere: gray matter $-(\mathrm{F}(2,84)=0.25, \mathrm{p}=0.78)$, white matter $-(\mathrm{F}(2,84)=0.75, \mathrm{p}=0.47), \operatorname{csf}-\mathrm{F}(2,84)=1.98, \mathrm{p}=0.15)$ we did not factor gray matter/ white matter/csf amounts into our analysis. 


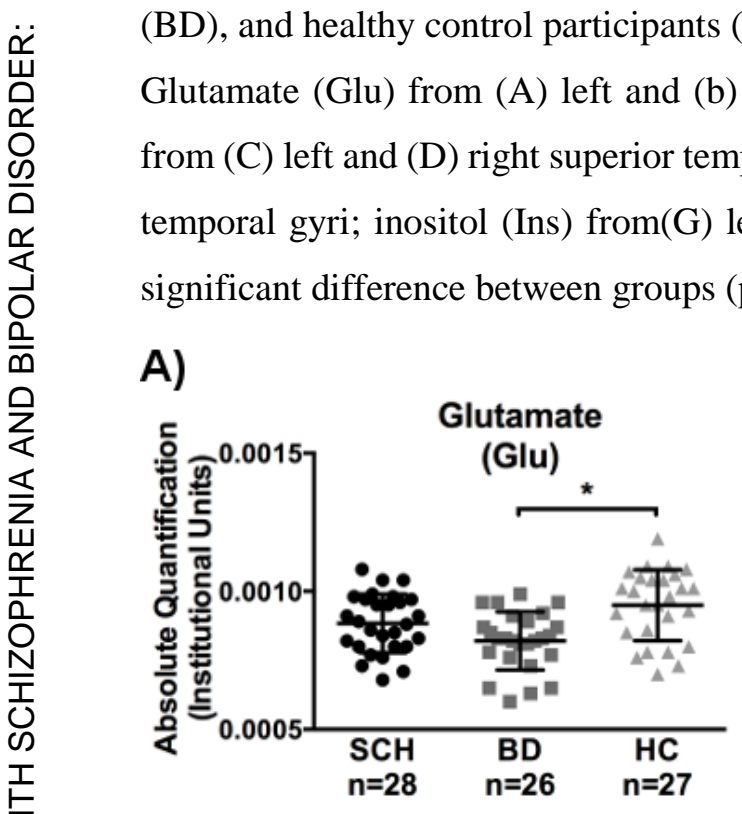

Figure 2. Absolute metabolite concentrations for patients with schizophrenia ( $\mathrm{SCH})$, bipolar disorder (BD), and healthy control participants (HC) acquired from the superior temporal gyrus using 1H MRS. (b) right superior temporal gyri; Total $\mathrm{N}$-acetyl aspartate (tNAA) from (C) left and (D) right superior temporal gyri; total creatine (tCr) from(E) left and (F) right superior emporal gyri; inositol (Ins) from(G) left and (H) right superior temporal gyri. * denotes statistically groups $(\mathrm{p}<0.008)$

B)
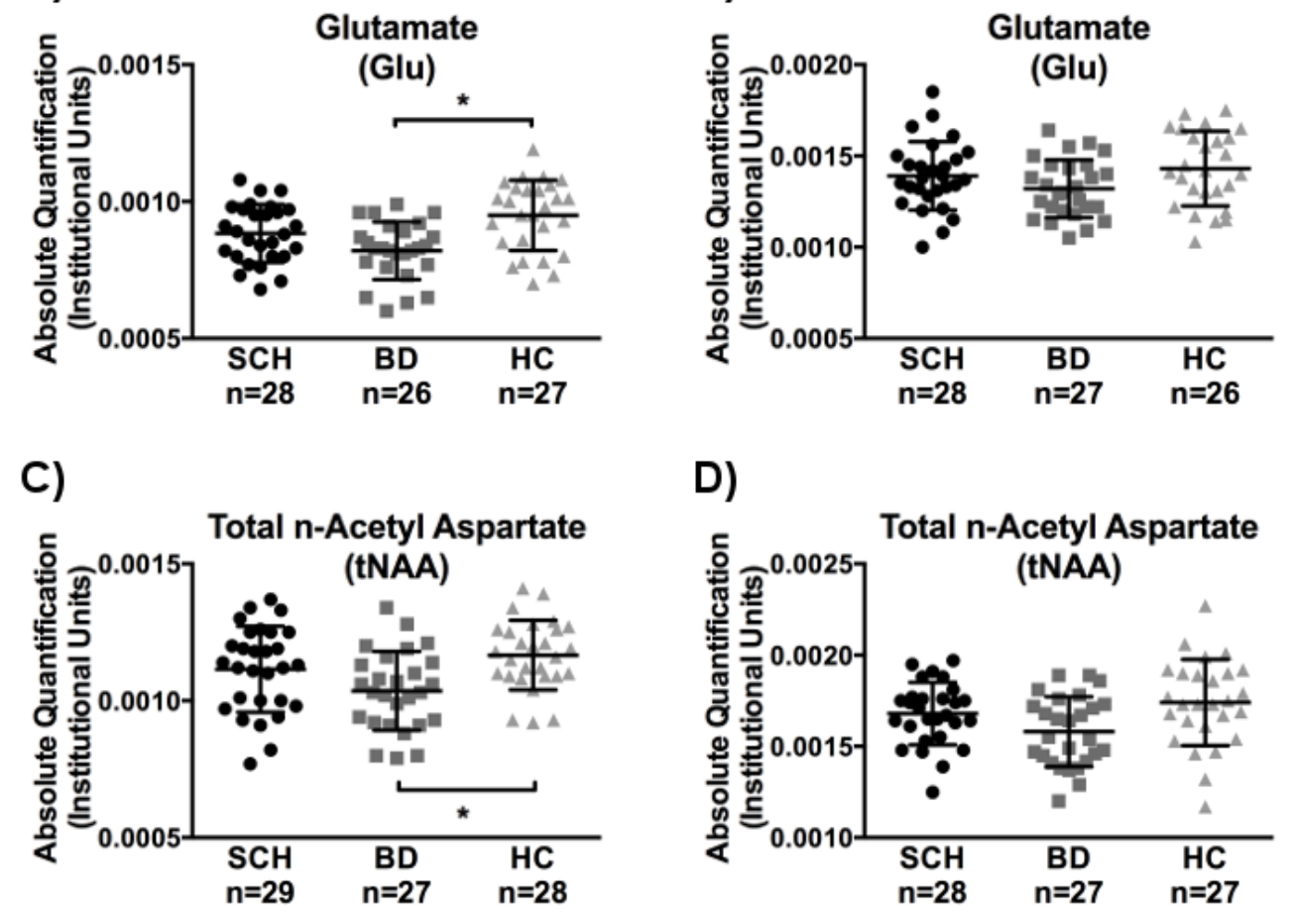

D)

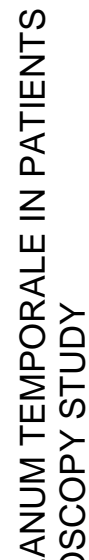

C)

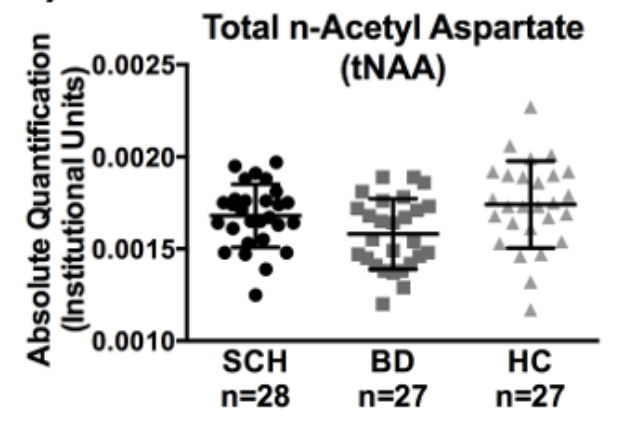

E)

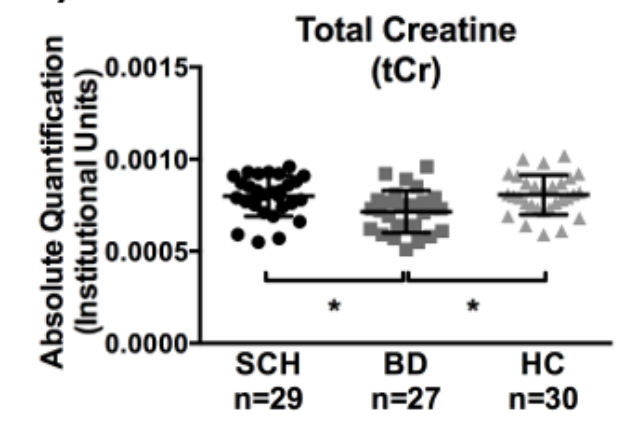

F)

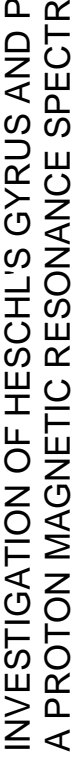
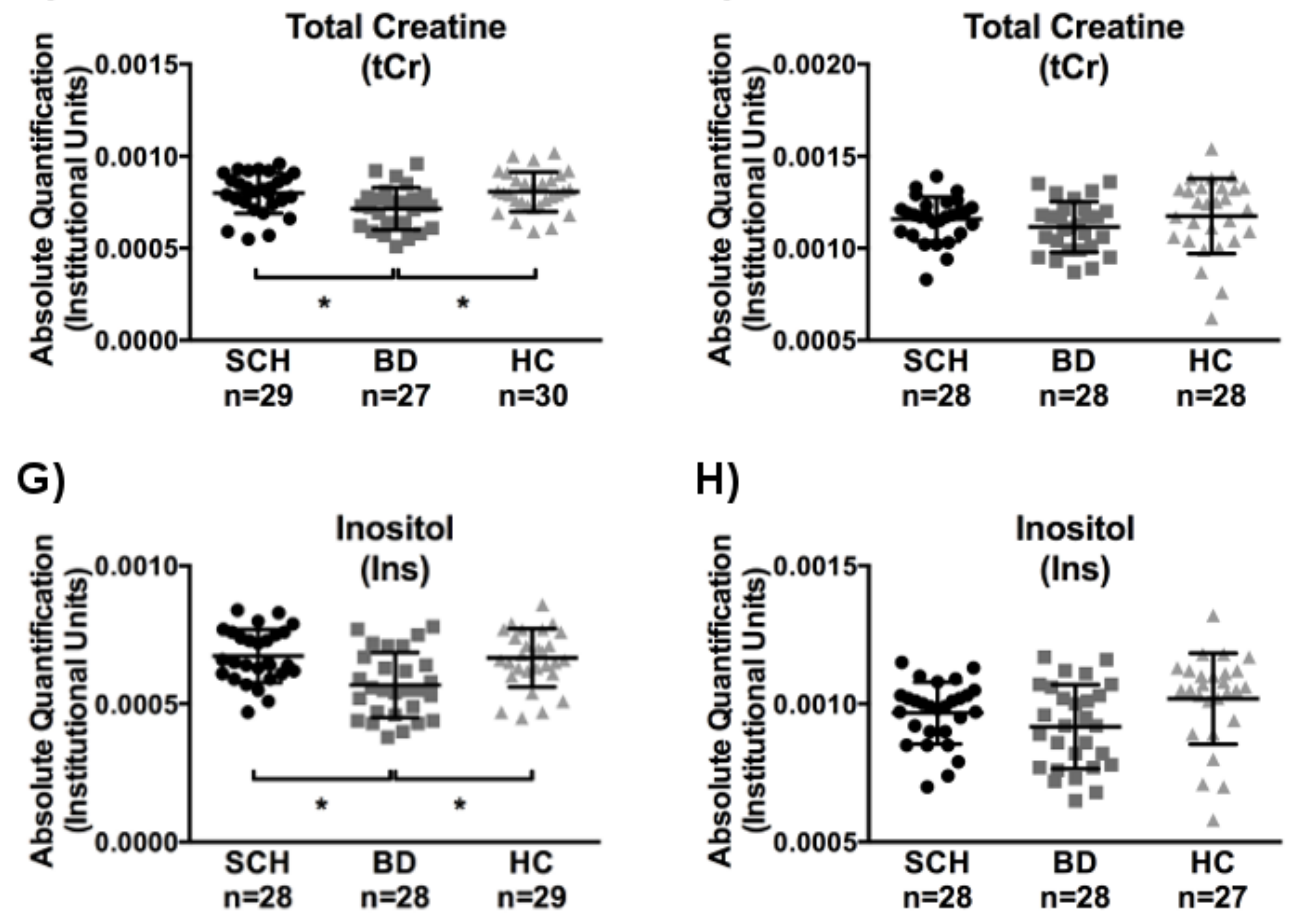


\subsection{Association between brain metabolites and clinical measures}

For schizophrenia patients, we performed exploratory correlation analyses to investigate the relationship between the brain metabolites and SAPS (total scores and subscores for bizarre behavior, formal thought disorder, inappropriate affect, delusion, and hallucination) and SANS (total scores and subscores for avolition, affective flattening, anhedonia, alogia, and attention). Glu showed relationships with SANS affective flattening $(r=0.44, p=0.02)$ and attention $(\mathrm{r}=0.44, \mathrm{p}=0.02)$ subscores, only for the right hemisphere. tNAA exhibited inverse relationships with SANS alogia $(r=-0.44, p=0.02)$ and attention subscores $(r=-0.44, p=0.02)$ in the left hemisphere. $\mathrm{tCr}$ had inverse correlations with SAPS total score $(r=-0.37, p=0.05)$, SAPS delusion subscore $(\mathrm{r}=-0.46, \mathrm{p}=0.01)$, SANS anhedonia $(\mathrm{r}=-0.46, \mathrm{p}=0.01)$, SANS alogia $(\mathrm{r}=-0.37, \mathrm{p}=0.05)$ in the left hemisphere. Ins showed inverse relationships with SAPS delusion subscore $(r=-0.42 \mathrm{p}=0.03)$, SANS anhedonia $(\mathrm{r}=-0.47, \mathrm{p}=0.01)$, and SANS alogia $(\mathrm{r}=-0.39$, $\mathrm{p}=0.04$ ) in the left hemisphere. For patients with bipolar disorder, we performed exploratory correlation analyses to investigate the relationship between the brain metabolites and YMRS, HDRS and BPRS. tNAA showed inverse relationships with HDRS for left $(r=-0.46, p=0.02)$ and right hemispheres $(\mathrm{r}=-0.41, \mathrm{p}=0.03) . \mathrm{tCr}$ also exhibited inverse correlation with HDRS for left $(r=-0.39, p=0.05)$ and right $(r=-0.44, p=0.02)$ hemispheres. In addition, Ins and HDRS had a reverse relationship in the right hemisphere $(\mathrm{r}=-0.52, \mathrm{p}=0.005)$.

\section{Discussion}

In this study, we observed decreased Glu concentrations within the left Heschl's gyrus and planum temporale of the superior temporal gyrus of patients with schizophrenia and bipolar disorder when compared to healthy participants. On the other hand, Glu, tNAA, $\mathrm{tCr}$ and Ins concentrations of the bipolar disorder group were lower when compared to schizophrenia patients and healthy participants. Moreover, $\mathrm{tCr}$ and Ins concentrations in the left superior temporal gyrus of schizophrenia. Patients were significantly higher than the concentrations measured from patients with bipolar disorder. All of these differences in metabolite levels between groups reached statistical significance only in the left superior temporal cortex, suggesting a possible location- specific abnormality within the dominant hemisphere of auditory cortices for schizophrenia and bipolar disorder patients. It has been shown that auditory cortices are functionally lateralized (Zatorre et al., 1992; Kell et al., 2011) and the 
auditory cortex of the dominant hemisphere is associated with psychiatric disorders (Dierks et al., 1999; Plaze et al., 2006; Shinn et al., 2013).

Previous studies have reported glutamatergic abnormalities related to clinical progression in schizophrenia observed within different brain regions such as the anterior cingulate cortex (Goff and Coyle, 2001; Clinton and Meador-Woodruff, 2004; Kugaya and Sanacora, 2005; Javitt et al., 2012). Specifically, schizophrenia patients have exhibited increased concentrations of glutamatergic metabolites if the patients were in a first-episode and conversely, decreased concentrations if the patients had a chronic disease (Ohrmann et al., 2005; Ohrmann et al., 2008; Marsman et al., 2013; Schwerk et al., 2014). A recent study, investigating schizophrenia patients showed elevated Gln and Gln-to-Glu ratio but no significant difference in Glu levels in patients when compared to healthy comparisons (Bustillo et al., 2014). Our findings in a cohort of chronic schizophrenia patients exhibited decreased Glu concentrations at trend level for schizophrenia patients when compared to healthy participants and no change for Glx concentrations were observed.

Previous reports did not find any difference in Glu concentrations between patients with bipolar disorder and healthy participants (Gigante et al., 2012); however they consistently showed increase in Glx concentration for bipolar disorder patients, regardless of mood state (Yuksel and Ongur, 2010; Gigante et al., 2012). Although alterations in Glx have been suggested to mostly reflect changes in Glu levels (Dager et al., 2004), previous reports are suggestive of abnormalities in other components of the composite Glx peak, such as Gln (Moore et al., 2007), rather than Glu. However, our findings showed significant difference in Glu levels but not in Glx concentrations.

Glutamate turnover may be altered in different ways, resulting in changes in Gln-to-Glu ratio, depending on the disease state, i.e. manic (Ongur et al., 2008) or depressive (Auer et al., 2000) phases of bipolar disorder (reviewed in (Yuksel and Ongur, 2010)). These findings suggest an alteration in glutamatergic dysfunction between mania and depression for bipolar disorder. Glial cells are responsible for Glu-Gln cycling (Tansey et al., 1991; Cooper et al., 2003); therefore glial abnormalities are potentially the basis of differences between mania and depression in bipolar disorder (Ongur et al., 1998). Unfortunately, the field strength of theMRI scanner used in the current study did not allow us to reliably quantify Gln concentrations. We can only speculate that since the Glx levels did not differ between groups, but Glu concentrations were lower for both schizophrenia and bipolar disorder patients when compared to healthy participants, Gln levels may have been elevated. Taken together, it is possible to 
suggest that abnormalities in the Gln/ Glu cycle might be a cause of the observed reduction in Glu levels for the patients with bipolar disorder.

NAA is known to be lower in schizophrenia and bipolar disorder patients (Steen et al., 2005; Yildiz-Yesiloglu and Ankerst, 2006; Kraguljac et al., 2012a,b). Our investigation did not reveal any significant abnormality in tNAA concentrations in schizophrenia patients, possibly due to medication effects (Szulc et al., 2011; Kraguljac et al., 2012a; Szulc et al., 2013), but exhibited reduced tNAA levels for bipolar patients. $\mathrm{tCr}$ and Ins levels remain unchanged in schizophrenia (Schwerk et al., 2014) and our findings confirm this statement. However, there are a few studies that found reduced $\mathrm{tCr}$ levels in dorsolateral prefrontal cortex, anterior cingulate cortex, hippocampus and basal ganglia of schizophrenia patients (Ohrmann et al., 2005; Ohrmann et al., 2007; Ongur et al., 2009). In addition, the literature is not conclusive about the concentrations of $\mathrm{Cr}$ and Ins for patients with bipolar disorder (Yildiz-Yesiloglu and Ankerst, 2006; Frey et al., 2007; Theberge et al., 2007; Ongur et al., 2009; Silverstone and McGrath, 2009; Kraguljac et al., 2012a; Sikoglu et al., 2013). The decrease in tNAA, tCr and Ins that we observed in patients with bipolar disorder compared to healthy participants may be a region specific disturbance in this disorder, as we investigated an understudied brain area, i.e. the left superior temporal cortex. Regardless, these abnormalities are suggestive of abnormalities in neurochemicals other than glutamate in bipolar disorder.

The comparison of findings from patients with schizophrenia and bipolar disorder revealed significant differences only for $\mathrm{tCr}$ and Ins levels. The auditory system may show different abnormalities at multiple levels both in schizophrenia and bipolar disorder (Skold et al., 2014). Our observations may imply that there exist common, i.e. glutamatergic dysfunction, as well as distinct abnormalities, i.e. in brain bioenergetics in schizophrenia and bipolar disorder within the auditory system.

Previous studies have focused primarily on higher order cortices, such as the frontal lobe where different channels of information converge and hence conduct serial and parallel processing together. However, lower order sensory areas, such as the auditory cortices have simpler receptive fields with only serial processing capabilities (Yamada and Wilber, 1990; Rauschecker and Scott, 2009; Homan et al., 2014). Given the fact that the pathophysiology of psychiatric disorders is so complicated and these disorders may have different influences on different neural systems and circuits, investigating the primary sensory areas may provide an advantage. Therefore, in this study we focused on the superior temporal gyrus to acquire $1 \mathrm{H}$ MRS data. 
Previous studies in the left STG in schizophrenia revealed correlations with clinical data, which suggests a relationship between the region and the effects of clinical factors. A 31P MRS study reported correlation between the hallucination score of the Positive and Negative Syndrome Scale (PANSS) and phosphomonoesters and phosphodiesters (Nenadic et al., 2014). A recent 1H MRS study comparing hallucinations in the STG reported that NAA/Cr ratio correlated with total and negative subscore of the PANSS in schizophrenia (Homan et al., 2014). Neuronal activation patterns for patients with auditory hallucinations were more prominent for the left hemisphere when compared to non-hallucinating patients as shown by functional neuroimaging studies with auditory tasks (Dierks et al., 1999; Shinn et al., 2013), which may indicate the relevance of the left auditory cortex. Besides, the auditory processing deficits in functional studies (Hall et al., 2009; Oribe et al., 2010; Domjan et al., 2012; Atagun et al., 2013; Atagun et al., 2014) point to abnormalities of the auditory cortices both in schizophrenia and bipolar disorder. In this study, both schizophrenia and bipolar disorder patients exhibited relationships between the studied metabolites and some of the clinical measures, especially on the left hemisphere. For example, HDRS scores correlated tCr and Ins concentrations in superior temporal cortices. This is particularly important as it may indicate a relationship between the metabolite levels and mood status.

\section{Limitations}

The current study had several limitations. First, our patients were patients with chronic disease and all were medicated. Although there was no statistically significant medication effect, small numbers of patients were taking each individual medication; therefore we cannot exclude medication effects conclusively. It is still possible that some of the metabolites were altered due to medications. Second, at $3 \mathrm{~T}$ magnetic field strength, some of the metabolite peaks in the $1 \mathrm{H}-$ MRS spectrum cannot be decomposed. In spite of the high quality of the glutamate output, it is difficult to completely discriminate glutamate from glutamine and GABA signal amplitudes, thus we could only identify Glu and Glx levels. Third, $8 \mathrm{~cm} 3$ voxel volumes cover an area larger than the auditory cortices alone.

\section{Conclusion}

Overlapping symptoms and intermediate diagnoses suggest that schizophrenia and bipolar disorder are disorders on a continuum. Genome wide association studies have identified shared 
risk genes in schizophrenia and bipolar disorder involving glutamatergic metabolism and cascades (see review; Coyle, 2006), which are in line with the glutamatergic abnormalities we have observed. Given that neurotransmission and intracellular functions are two major functions of glutamatergic metabolites, future studies focusing on glutamatergic abnormalities may provide novel targets for treatment development for both schizophrenia and bipolar disorder. In addition, this study reports a significant difference between creatine and inositol levels in patients with schizophrenia and bipolar disorder in the left superior temporal region. Taken together, there may be both shared and distinct mechanistic abnormalities within auditory cortices underlying pathophysiologies of schizophrenia and bipolar disorder. Therefore, our findings may suggest biological markers that may quantitatively distinguish the two disorders, and might be of importance for future studies.

\section{Role of funding source}

The study was funded by the Scientific Research Projects Committee of the Y1ldirım Beyazit University (project \#803) and NIMH grants to CMM (MH073998) and to DÖ (MH094594).

\section{References}

Andreasen, N.C., 1983. The Scale for Assessment of Negative Symptoms (SANS). University of Iowa, Iowa City, IA.

Andreasen, N.C., 1984. The Scale for the Assessment of Positive Symptoms (SAPS). University of Iowa, Iowa City, IA.

Arnone, D., Cavanagh, J., Gerber, D., Lawrie, S.M., Ebmeier, K.P., McIntosh, A.M., 2009. Magnetic resonance imaging studies in bipolar disorder and schizophrenia: metaanalysis.

Br. J. Psychiatry 195 (3), 194-201.

Atagun, M.I., Guntekin, B., Ozerdem, A., Tulay, E., Basar, E., 2013. Decrease of theta response in euthymic bipolar patients during an oddball paradigm. Cogn. Neurodyn. 7 (3), 213-223.
Atagun, M.I., Guntekin, B., Masali, B., Tulay, E., Basar, E., 2014. Decrease of eventrelated delta oscillations in euthymic patients with bipolar disorder. Psychiatry Res. 223 (1), 43-48.

Auer, D.P., Putz, B., Kraft, E., Lipinski, B., Schill, J., Holsboer, F., 2000. Reduced glutamate in the anterior cingulate cortex in depression: an in vivo proton magnetic resonance spectroscopy study. Biol. Psychiatry 47 (4), 305-313.

Bramon, E., Sham, P.C., 2001. The common genetic liability between schizophrenia and bipolar disorder: a review. Curr. Psychiatry Rep. 3 (4), 332-337.

Brugger, S., Davis, J.M., Leucht, S., Stone, J.M., 2011. Proton magnetic resonance spectroscopy and illness stage in schizophrenia - a systematic review 
andmeta-analysis. Biol. Psychiatry 69 (5), 495-503.

Bustillo, J.R., Chen, H., Jones, T., Lemke, N., Abbott, C., Qualls, C., Canive, J., Gasparovic, C., 2014. Increased glutamine in patients undergoing long-term treatment for schizophrenia: a proton magnetic resonance spectroscopy study at $3 \mathrm{~T}$. JAMA Psychiatry 71 (3), 265-272.

Clinton, S.M., Meador-Woodruff, J.H., 2004. Abnormalities of the NMDA receptor and associated intracellular molecules in the thalamus in schizophrenia and bipolar disorder. Neuropsychopharmacology 29 (7), 1353-1362.

Colla, M., Schubert, F., Bubner, M., Heidenreich, J.O., Bajbouj, M., Seifert, F., Luborzewski, A., Heuser, I., Kronenberg, G., 2009. Glutamate as a spectroscopicmarker of hippocampal structural plasticity is elevated in long-term euthymic bipolar patients on chronic lithium therapy and correlates inversely with diurnal cortisol. Mol. Psychiatry 14 (7), 696-704 (647).

Cooper, J., Bloom, F., Roth, R., 2003. The Biochemical Basis of Neuropharmacology. Oxford University Press, New York.

Coyle, J.T., 2006. Glutamate and schizophrenia: beyond the dopamine hypothesis. Cell. Mol. Neurobiol. 26 (4-6), 365-384.

Craddock, N., Owen, M.J., 2005. The beginning of the end for the Kraepelinian dichotomy. Br. J. Psychiatry 186, 364-366.

Cuthbert, B.N., 2014. The RDoC framework: facilitating transition from ICD/DSM to dimensional approaches that integrate neuroscience and psychopathology. World Psychiatry 13 (1), 28-35.
Dager, S.R., Friedman, S.D., Parow, A., Demopulos, C., Stoll, A.L., Lyoo, I.K., Dunner, D.L., Renshaw, P.F., 2004. Brain metabolic alterations in medication-free patients with bipolar disorder. Arch. Gen. Psychiatry 61 (5), 450-458.

Dierks, T., Linden, D.E., Jandl,M., Formisano, E., Goebel, R., Lanfermann, H., Singer,W., 1999. Activation of Heschl's gyrus during auditory hallucinations. Neuron 22 (3), 615621.

Domjan, N., Csifcsak, G., Drotos, G., Janka, Z., Szendi, I., 2012. Different patterns of auditory information processing deficits in chronic schizophrenia and bipolar disorder with psychotic features. Schizophr. Res. 139 (1-3), 253-259.

Ethridge, L.E., Hamm, J.P., Shapiro, J.R., Summerfelt, A.T., Keedy, S.K., Stevens,M.C., Pearlson, G., Tamminga, C.A., Boutros, N.N., Sweeney, J.A., Keshavan, M.S., Thaker, G., Clementz, B.A., 2012. Neural activations during auditory oddball processing discriminating schizophrenia and psychotic bipolar disorder. Biol. Psychiatry 72 (9), 766-774.

First, M.D., Gibbon, M., Spitzer, R.L., Gibbon, M., Williams, J.B.W., 1996. User's Guide for the Structured Interview for DSM-IV Axis I Disorders Research Version (SCID-I, version 2.0). Biometrics Research, New York.

Frey, B.N., Stanley, J.A., Nery, F.G., Monkul, E.S., Nicoletti, M.A., Chen, H.H., Hatch, J.P.,

Caetano, S.C., Ortiz, O., Kapczinski, F., Soares, J.C., 2007. Abnormal cellular energy and phospholipid metabolism in the left dorsolateral prefrontal cortex of medicationfree individuals with bipolar disorder: an in vivo 1H MRS study. Bipolar Disord. 9 (Suppl. 1), 119-127. 
Gigante, A.D., Bond, D.J., Lafer, B., Lam, R.W., Young, L.T., Yatham, L.N., 2012. Brain glutamate levels measured by magnetic resonance spectroscopy in patients with bipolar disorder: a meta-analysis. Bipolar Disord. 14 (5), 478-487.

Goff, D.C., Coyle, J.T., 2001. The emerging role of glutamate in the pathophysiology and treatment of schizophrenia. Am. J. Psychiatry 158 (9), 1367-1377.

Hall, M.H., Schulze, K., Rijsdijk, F., Kalidindi, S., McDonald, C., Bramon, E., Murray, R.M.,

Sham, P., 2009. Are auditory P300 and duration MMN heritable and putative endophenotypes of psychotic bipolar disorder? A Maudsley Bipolar Twin and Family Study. Psychol. Med. 39 (8), 12771287.

Hamilton, M., 1960. A rating scale for depression. J. Neurol. Neurosurg. Psychiatry $23,56-62$.

Homan, P., Vermathen, P., Van Swam, C., Federspiel, A., Boesch, C., Strik, W., Dierks, T., Hubl, D., Kreis, R., 2014. Magnetic resonance spectroscopy investigations of functionally defined language areas in schizophrenia patients with and without auditory hallucinations. NeuroImage 94, $23-$ 32.

Jardri, R., Pouchet, A., Pins, D., Thomas, P., 2011. Cortical activations during auditory verbal hallucinations in schizophrenia: a coordinate-based meta-analysis. Am. J. Psychiatry 168 (1), 73-81.

Javitt, D.C., 2009. When doors of perception close: bottom-up models of disrupted cognition in schizophrenia. Annu. Rev. Clin. Psychol. 5, 249-275.

Javitt, D.C., 2010.When doors of perception close: bottom-up models of disrupted cognition in schizophrenia. Annu. Rev. Clin. Psychol. 5, 249-275.

Javitt, D.C., Zukin, S.R., Heresco-Levy, U., Umbricht, D., 2012. Has an angel shown the way? Etiological and therapeutic implications of the PCP/NMDA model of schizophrenia. Schizophr. Bull. 38 (5), 958 966.

Kell, C.A., Morillon, B., Kouneiher, F., Giraud, A.L., 2011. Lateralization of speech production starts in sensory cortices - a possible sensory origin of cerebral left dominance for speech. Cereb. Cortex 21 (4), 932-937.

Kempton, M.J., Geddes, J.R., Ettinger, U., Williams, S.C., Grasby, P.M., 2008. Metaanalysis, database, and meta-regression of 98 structural imaging studies in bipolar disorder. Arch. Gen. Psychiatry 65 (9), 1017-1032.

Keshavan, M.S., Ongur, D., 2014. The journey from RDC/DSM diagnoses toward RDoC dimensions. World Psychiatry 13 (1), 44-46.

Kraguljac, N.V., Reid, M., White, D., Jones, R., den Hollander, J., Lowman, D., Lahti, A.C., 2012a. Neurometabolites in schizophrenia and bipolar disorder - a systematic review and meta-analysis. Psychiatry Res. 203 (23), 111-125.

Kraguljac, N.V., Reid, M.A., White, D.M., den Hollander, J., Lahti, A.C., 2012b. Regional decoupling of $\mathrm{N}$-acetyl-aspartate and glutamate in schizophrenia. Neuropsychopharmacology 37 (12), 26352642.

Kugaya, A., Sanacora, G., 2005. Beyondmonoamines: glutamatergic function in mood disorders. CNS Spectr. 10 (10), 808-819.

Kuhn, S., Gallinat, J., 2012. Quantitative metaanalysis on state and trait aspects of auditory 
verbal hallucinations in schizophrenia. Schizophr. Bull. 38 (4), 779-786.

Machado-Vieira, R., Ibrahim, L., Henter, I.D., Zarate Jr., C.A., 2012. Novel glutamatergic agents for major depressive disorder and bipolar disorder. Pharmacol. Biochem. Behav. 100 (4), 678-687.

Marsman, A., van den Heuvel, M.P., Klomp, D.W., Kahn, R.S., Luijten, P.R., Hulshoff Pol, H.E., 2013. Glutamate in schizophrenia: a focused review and meta-analysis of (1) $\mathrm{H}-$ MRS studies. Schizophr. Bull. 39 (1), 120129.

Modinos, G., Costafreda, S.G., van Tol, M.J., McGuire, P.K., Aleman, A., Allen, P., 2013. Neuroanatomy of auditory verbal hallucinations in schizophrenia: a quantitative metaanalysis of voxel-based morphometry studies. Cortex 49 (4), 10461055.

Moore, C.M., Frazier, J.A., Glod, C.A., Breeze, J.L., Dieterich, M., Finn, C.T., Frederick, B., Renshaw, P.F., 2007. Glutamine and glutamate levels in children and adolescents with bipolar disorder: a 4.0-T proton magnetic resonance spectroscopy study of the anterior cingulate cortex. J. Am. Acad. Child Adolesc. Psychiatry 46 (4), 524-534.

Nenadic, I., Dietzek, M., Langbein, K., Rzanny, R., Gussew, A., Reichenbach, J.R., Sauer, H.,

Smesny, S., 2014. Superior temporal metabolic changes related to auditory hallucinations: a (31)P-MR spectroscopy study in antipsychotic-free schizophrenia patients. Brain Struct. Funct. 219 (5), 1869-1872.

Ohrmann, P., Siegmund, A., Suslow, T., Spitzberg, K., Kersting, A., Arolt, V., Heindel, W., Pfleiderer, B., 2005. Evidence for glutamatergic neuronal dysfunction in the prefrontal cortex in chronic but not in first-episode patients with schizophrenia: a proton magnetic resonance spectroscopy study. Schizophr. Res. 73 (2-3), 153-157.

Ohrmann, P., Siegmund, A., Suslow, T., Pedersen, A., Spitzberg, K., Kersting, A., Rothermundt, M., Arolt, V., Heindel, W., Pfleiderer, B., 2007. Cognitive impairment and in vivo metabolites in first-episode neuroleptic-naive and chronic medicated schizophrenic patients: a proton magnetic resonance spectroscopy study. J. Psychiatr. Res. 41 (8), 625-634.

Ohrmann, P., Kugel, H., Bauer, J., Siegmund, A., Kolkebeck, K., Suslow, T., Wiedl, K.H., Rothermundt, M., Arolt, V., Pedersen, A., 2008. Learning potential on the WCST in schizophrenia is related to the neuronal integrity of the anterior cingulate cortex as measured by proton magnetic resonance spectroscopy. Schizophr. Res. 106 (2-3), 156-163.

Oldfield, R.C., 1971. The assessment and analysis of handedness: the Edinburgh inventory. Neuropsychologia 9 (1), 97-113.

Ongur, D., Drevets, W.C., Price, J.L., 1998. Glial reduction in the subgenual prefrontal cortex in mood disorders. Proc. Natl. Acad. Sci. U. S. A. 95 (22), 13290-13295.

Ongur, D., Jensen, J.E., Prescot, A.P., Stork, C., Lundy, M., Cohen, B.M., Renshaw, P.F., 2008. Abnormal glutamatergic neurotransmission and neuronal-glial interactions in acute mania. Biol. Psychiatry 64 (8), 718-726.

Ongur, D., Prescot, A.P., Jensen, J.E., Cohen, B.M., Renshaw, P.F., 2009. Creatine abnormalities in schizophrenia and bipolar disorder. Psychiatry Res. 172 (1), 44-48. Ongur, D., Haddad, S., Prescot, A.P., Jensen, J.E., Siburian, R., Cohen, B.M., Renshaw, P.F., Smoller, J.W., 2011. Relationship between genetic variation in the glutaminase gene GLS1 and brain 
glutamine/glutamate ratio measured in vivo. Biol. Psychiatry 70 (2), 169-174.

Oribe, N., Onitsuka, T., Hirano, S., Hirano, Y., Maekawa, T., Obayashi, C., Ueno, T., Kasai, K., Kanba, S., 2010. Differentiation between bipolar disorder and schizophrenia revealed by neural oscillation to speech sounds: an MEG study. Bipolar Disord. 12 (8), 804812.

Overall, J.E., Gorham, D.R., 1962. The brief psychiatric rating scale. Psychological Reports 10, 799-812.

Paz, R.D., Tardito, S., Atzori, M., Tseng, K.Y., 2008. Glutamatergic dysfunction in schizophrenia: from basic neuroscience to clinical psychopharmacology. Eur. Neuropsychopharmacol. 18 (11), 773-786.

Plaze, M., Bartres-Faz, D., Martinot, J.L., Januel, D., Bellivier, F., De Beaurepaire, R., Chanraud, S., Andoh, J., Lefaucheur, J.P., Artiges, E., Pallier, C., Paillere-Martinot, M.L., 2006. Left superior temporal gyrus activation during sentence perception negatively correlates with auditory hallucination severity in schizophrenia patients. Schizophr. Res. 87 (1-3), 109-115.

Poels, E.M., Kegeles, L.S., Kantrowitz, J.T., Javitt, D.C., Lieberman, J.A., Abi-Dargham, A.,

Girgis, R.R., 2014a. Glutamatergic abnormalities in schizophrenia: a review of proton MRS findings. Schizophr. Res. 152 (2-3), 325-332.

Poels, E.M., Kegeles, L.S., Kantrowitz, J.T., Slifstein, M., Javitt, D.C., Lieberman, J.A., Abi- Dargham, A., Girgis, R.R., 2014b. Imaging glutamate in schizophrenia: review of findings and implications for drug discovery. Mol. Psychiatry 19 (1), 20-29.

Provencher, S.W., 1993. Estimation of metabolite concentrations from localized in vivo proton NMR spectra. Magn. Reson. Med. 30 (6), 672-679.

Provencher, S.W., 2001. Automatic quantitation of localized in vivo $1 \mathrm{H}$ spectra with LCModel. NMR Biomed. 14 (4), 260264.

Rajarethinam, R.P., DeQuardo, J.R., Nalepa, R., Tandon, R., 2000. Superior temporal gyrus in schizophrenia: a volumetric magnetic resonance imaging study. Schizophr. Res. 41 (2), 303-312.

Rauschecker, J.P., Scott, S.K., 2009. Maps and streams in the auditory cortex: nonhuman primates illuminate human speech processing. Nat. Neurosci. 12 (6), 718-724.

Sanacora, G., Zarate, C.A., Krystal, J.H., Manji, H.K., 2008. Targeting the glutamatergic system to develop novel, improved therapeutics for mood disorders. Nat. Rev. Drug Discov. 7 (5), 426-437.

Schifitto, G., Zhong, J., Gill, D., Peterson, D.R., Gaugh, M.D., Zhu, T., Tivarus, M., Cruttenden, K., Maggirwar, S.B., Gendelman, H.E., Dewhurst, S., Gelbard, H.A., 2009. Lithium therapy for human immunodeficiency virus type 1-associated neurocognitive impairment. J. Neurovirol. 15 (2), 176-186.

Schwerk, A., Alves, F.D., Pouwels, P.J., van Amelsvoort, T., 2014.Metabolic alterations associated with schizophrenia: a critical evaluation of proton magnetic resonance spectroscopy studies. J. Neurochem. 128 (1), $1-87$.

Seese, R.R., O'Neill, J., Hudkins, M., Siddarth, P., Levitt, J., Tseng, B., Wu, K.N., Caplan, R.,

2011. Proton magnetic resonance spectroscopy and thought disorder in childhood schizophrenia. Schizophr. Res. 133 (1-3), 82-90. 
Shenton, M.E., Kikinis, R., Jolesz, F.A., Pollak, S.D., LeMay, M., Wible, C.G., Hokama, H., Martin, J., Metcalf, D., Coleman, M., et al., 1992. Abnormalities of the left temporal lobe and thought disorder in schizophrenia. A quantitative magnetic resonance imaging study. N. Engl. J. Med. 327 (9), 604-612.

Shinn, A.K., Baker, J.T., Cohen, B.M., Ongur, D., 2013. Functional connectivity of left Heschl's gyrus in vulnerability to auditory hallucinations in schizophrenia. Schizophr. Res. 143 (2-3), 260-268.

Sikoglu, E.M., Jensen, J.E., Vitaliano, G., Liso Navarro, A.A., Renshaw, P.F., Frazier, J.A., Moore, C.M., 2013. Bioenergetic measurements in children with bipolar disorder: a pilot $31 \mathrm{P}$ magnetic resonance spectroscopy study. PLoS One 8 (1), e54536.

Silverstone, P.H.,McGrath, B.M., 2009. Lithium and valproate and their possible effects on the myo-inositol second messenger system in healthy volunteers and bipolar patients. Int. Rev. Psychiatry 21 (4), 414-423.

Skold,M., Kallstrand, J., Nehlstedt, S., Nordin, A., Nielzen, S., Holmberg, J., Adolfsson, R., 2014. Thalamocortical abnormalities in auditory brainstem response patterns distinguish DSM-IV bipolar disorder type I from schizophrenia. J. Affect. Disord. 169, $105-111$.

Skolnick, P., Popik, P., Trullas, R., 2009. Glutamate-based antidepressants: 20 years on. Trends Pharmacol. Sci. 30 (11), 563569.

Soeiro-de-Souza,M.G., Salvadore, G., Moreno, R.A., Otaduy, M.C., Chaim, K.T., Gattaz, W.F., Zarate Jr., C.A., Machado-Vieira, R., 2013. Bcl-2 rs956572 polymorphism is associated with increased anterior cingulate cortical glutamate in euthymic bipolar I disorder. Neuropsychopharmacology 38 (3), 468-475.

Steen, R.G., Hamer, R.M., Lieberman, J.A., 2005. Measurement of brain metabolites by $1 \mathrm{H}$ magnetic resonance spectroscopy in patients with schizophrenia: a systematic review and meta-analysis. Neuropsychopharmacology 30 (11), 19491962.

Szulc, A., Galinska, B., Tarasow, E., Waszkiewicz, N., Konarzewska, B., Poplawska, R., Bibulowicz, D., Simonienko, K., Walecki, J., 2011. Proton magnetic resonance spectroscopy study of brain metabolite changes after antipsychotic treatment. Pharmacopsychiatry 44 (4), 148157.

Szulc, A., Galinska-Skok, B., Waszkiewicz, N., Bibulowicz, D., Konarzewska, B., Tarasow, E., 2013. Proton magnetic resonance spectroscopy changes after antipsychotic treatment. Curr. Med. Chem. 20 (3), 414427.

Takahashi, T., Suzuki, M., Zhou, S.Y., Tanino, R., Hagino, H., Kawasaki, Y., Matsui, M., Seto, H., Kurachi, M., 2006. Morphologic alterations of the parcellated superior temporal gyrus in schizophrenia spectrum. Schizophr. Res. 83 (2-3), 131-143.

Tansey, F.A., Farooq, M., Cammer, W., 1991. Glutamine synthetase in oligodendrocytes and astrocytes: new biochemical and immunocytochemical evidence. J. Neurochem. 56 (1), 266-272.

Theberge, J., Jensen, J.E., Rowland, L.M., 2007. Regarding "Increased prefrontal and hippocampal glutamate concentration in schizophrenia: evidence from a magnetic resonance spectroscopy study". Biol. Psychiatry 61 (10), 1218-1219 (author reply 1219-1220). 
Umbricht, D., Krljes, S., 2005. Mismatch negativity in schizophrenia: a meta-analysis. Schizophr. Res. 76 (1), 1-23.

Vita, A., De Peri, L., Deste, G., Sacchetti, E., 2012. Progressive loss of cortical gray matter in

schizophrenia: a meta-analysis and metaregression of longitudinal MRI studies. Transl. Psychiatry 2, e190.

Yamada, M., Wilber, J.F., 1990. Reciprocal regulation of preprothyrotropin-releasing hormone (TRH) mRNA in the rat anterior hypothalamus by thyroid hormone: dissociation from TRH concentrations during hypothyroidism. Neuropeptides 15 (1), 49-53.

Yatham, L.N., Kennedy, S.H., Schaffer, A., Parikh, S.V., Beaulieu, S., O'Donovan, C.,MacQueen, G.,McIntyre, R.S., Sharma, V., Ravindran, A., Young, L.T., Young, A.H., Alda,M.,Milev, R., Vieta, E., Calabrese, J.R., Berk, M., Ha, K., Kapczinski, F., 2009. Canadian Network for Mood and Anxiety Treatments (CANMAT) and International Society for Bipolar
Disorders (ISBD) collaborative update of CANMAT guidelines for the management of patients with bipolar disorder: update 2009. Bipolar Disord. 11 (3), 225-255.

Yildiz-Yesiloglu, A., Ankerst, D.P., 2006. Neurochemical alterations of the brain in bipolar disorder and their implications for pathophysiology: a systematic review of the in vivo proton magnetic resonance spectroscopy findings. Prog. Neuropsychopharmacol. Biol. Psychiatry 30 (6), 969-995.

Young, R.C., Biggs, J.T., Ziegler, V.E., Meyer, D.A., 1978. A rating scale for mania: reliability, validity and sensitivity. Br. J. Psychiatry 133, 429-435.

Yuksel, C., Ongur, D., 2010.Magnetic resonance spectroscopy studies of glutamate-related abnormalities in mood disorders. Biol. Psychiatry 68 (9), 785-794.

Zatorre, R.J., Evans, A.C., Meyer, E., Gjedde, A., 1992. Lateralization of phonetic and pitch discrimination in speech processing. Science 256 (5058), 846-849 


\section{CHAPTER 7}

\section{Neurochemical Differences Between Bipolar Disorder Type I and II in Superior Temporal Cortices: A Proton Magnetic Resonance Spectroscopy Study}

Murat IIlhan Atagün, Elif Muazzez Şıkoğlu, Serdar Süleyman Can, Görkem Karakaş Uğurlu, Semra Ulusoy Kaymak, Ali Çayköylü, Oktay Algın, Mary L Phillips, Constance M Moore, Dost Öngür

Journal of Affective Disorders 2018; 235: 15-19 


\section{Abstract}

\section{Background}

Despite the diagnostic challenges in categorizing bipolar disorder subtypes, bipolar I and II disorders (BD-I and BD-II respectively) are valid indices for researchers. Subtle neurobiological differences may underlie clinical differences between mood disorder subtypes. The aims of this study were to investigate neurochemical differences between bipolar disorder subtypes.

\section{Methods}

Euthymic BD-II patients $(\mathrm{n}=21)$ are compared with BD-I $(\mathrm{n}=28)$ and healthy comparison subjects (HCs, n=30). Magnetic Resonance Imaging (MRI) and proton spectroscopy ( ${ }^{1} \mathrm{H}$ MRS) were performed on a 3T Siemens Tim Trio system. MRS voxels were located in the left/right superior temporal cortices, and spectra acquired with the single voxel Point REsolved Spectroscopy Sequence (PRESS). The spectroscopic data were analyzed with LCModel (Version 6.3.0) software.

\section{Results}

There were significant differences between groups in terms of glutamate $[\mathrm{F}=6.27, \mathrm{p}=0.003]$, glutamate+glutamine $[\mathrm{F}=6.08, \mathrm{p}=0.004]$, inositol containing compounds (Ino) ( $\mathrm{F}=9.25$, $\mathrm{p}<0.001)$, NAA $[\mathrm{F}=7.63, \mathrm{p}=0.001]$ and creatine+phosphocreatine $[\mathrm{F}=11.06, \mathrm{p}<0.001]$ in the left hemisphere and Ino $[\mathrm{F}=5.65, \mathrm{p}=0.005]$ in the right hemisphere. Post-hoc comparisons showed that the BD-I disorder group had significantly lower metabolite levels in comparison to the BDII and the HC groups.

\section{Limitations}

This was a cross-sectional study with a small sample size. In addition, patients were on various psychotropic medications, which may have impacted the results.

\section{Conclusions}

Neurochemical levels, in the superior temporal cortices, measured with ${ }^{1} \mathrm{H}-\mathrm{MRS}$ discriminated between BD-II and BD-I. Although further studies are needed, one may speculate that the superior temporal cortices (particularly left hemispheric) play a critical role, whose pathology may be related to subtyping bipolar disorder.

Key words: Magnetic resonance spectroscopy, bipolar disorder, superior temporal gyrus 


\section{Introduction}

Despite the diagnostic challenges in categorizing bipolar disorder subtypes, bipolar I disorder (BD-I) and bipolar II disorder (BD-II) are valid categorical indices for researchers (Phillips and Kupfer, 2013; Parker et al., 2014). Along with depressive episodes, presence of manic or hypomanic episodes is the major difference between BD-I and BD-II disorders. Despite the valid diagnostic boundaries, only limited studies have reported neurobiological differences between the subtypes (Phillips and Kupfer, 2013; Parker et al., 2014; McGrath et al., 2014).

Genetic studies have identified differences between BD-I and BD-II (Lee et al., 2011; Uemura et al., 2011). Psychosis (Parker et al., 2014), neurotoxic processes (Yumru et al., 2009; Uemura et al., 2011) and cognitive dysfunction (Bora et al., 2011) are more prevalent in BD-I compared to BD-II. Magnetic resonance imaging (MRI) studies have reported that BD-I had more severe findings in comparison to BD-II in volumes (Hauser et al., 2000), white matter lesions (Ambrosi et al., 2013) and functional connectivity (Li et al., 2012). Neurochemistry may also differ between the subtypes.

Proton magnetic resonance spectroscopy $\left({ }^{1} \mathrm{H}-\mathrm{MRS}\right)$ can quantify specific neurochemical compounds in the brain in vivo. It has been observed in ${ }^{1} \mathrm{H}-\mathrm{MRS}$ that mood state shifts and medications alter metabolites in BD-I (Moore et al., 2007). However, only a few MRS studies have compared BD-I and BD-II. No consistent finding has been reported between the subtypes in the frontal (Winsberg et al., 2000) or temporal (Hamakawa et al., 1998; Atagun et al., 2017) lobes, or the basal ganglia (Silverstone et al., 2004). Only one study detected increased choline concentrations in the basal ganglia in BD-II in comparison to BD-I (Kato et al., 1996).

Patients with bipolar disorder showed fronto-temporal dysconnectivity (Ozerdem et al., 2011) and cortical thinning was specific to superior temporal cortex in BD-I (Hanford et al., 2016). Furthermore, auditory processing deficits in bipolar I disorder are related to left hemisphere superior temporal cortices (McCarley et al., 2008). Moreover, it has been postulated that shared neurochemical abnormalities in superior temporal cortices by schizophrenia and bipolar disorder might be a common feature of psychosis (Nudmamud et al., 2003). On the other hand, superior temporal cortices may have unique local metabolic disturbances in both schizophrenia and bipolar disorder (McNamara et al., 2014). These findings have highlighted the pathology of superior temporal lobes in bipolar disorder. However, it is not clear whether there is difference between subtypes of bipolar disorder. 
In this study, we aimed to compare neurochemical differences between BD-I and BD-II disorders utilizing ${ }^{1} \mathrm{H}-\mathrm{MRS}$ in superior temporal cortices. Our hypothesis was that there might be neurochemical differences between the subtypes of bipolar disorder. To our knowledge, this is the first ${ }^{1} \mathrm{H}-\mathrm{MRS}$ study to assess superior temporal lobes in BD-I and BD-II. In vivo assessment of metabolite levels in superior temporal lobes may show differences in pathogenesis of the subtypes of bipolar disorder.

\section{Methods}

All participants provided a written informed consent before study participation. This study was approved by the local Ethical Committee. In a recent article, we have reported abnormal metabolite levels in the superior temporal lobes of participants with BD-I (Atagün et al., 2015). We further enrolled patients with BD-II ( $\mathrm{n}=21$, mean age $=38.38 \pm 13.84,12$ women/ 9 men and compared with our BD-I sample and healthy comparison subjects (HCs). All patients were euthymic during the study participation. Clinical evaluation tools were Structured Clinical Interview according to the DSM-IV (SCID-I) (First et al., 1996), Young Mania Rating Scale (YMRS) (Young et al., 1978) and Hamilton Depression Rating Scale (HDRS) (Hamilton, 1960).

Data acquisition processes were performed with a 3.0 T Siemens MAGNETOM TIM Trio MR system (Siemens, Erlangen, Germany) and a 32 channel phased-array head coil. $\mathrm{T}_{1}$ anatomical MRI [MPRAGE sequence, parameters were TE: 3.02, TR: $2000 \mathrm{~ms}$, FOV phase: 100, FOV read: 215, slice thickness: 0.84, number of slices: 192] and spectroscopy voxels [single voxel Point REsolved Spectroscopy Sequence (PRESS); $8 \mathrm{~cm}^{3}$ voxels $(2 \mathrm{~cm} \mathrm{X} 2 \mathrm{~cm} \mathrm{X} 2 \mathrm{~cm}$ ); TE: 30 ms, TR: $2000 \mathrm{~ms}, 128$ averages] were recorded to evaluate left and right hemisphere superior temporal lobes which host Heschl's gyrus and planum temporale (Figure 1a). B $\mathrm{B}_{0}$ shimming was manually applied around the voxel. LCModel software (Version 6.3.0) was used to quantify the metabolite levels (Provencher, 2001), using water as internal reference. Representative spectra (Figure 1b) and quantified metabolites are presented in the supplement. Gray matter, white matter and cerebro-spinal fluid (CSF) contributions to the voxels were analyzed in T1 weighted structural MRI with Statistical Parametric Mapping-8 (SPM-8) software [Welcome Department of Imaging Neuroscience, London, UK; (http://www.fil.ion.ucl.ac.uk/spm/software/spm8/)] (for further details please see Atagün et al., 2015). Alanine, aspartate, creatine and phosphocreatine (Cr), $\gamma$-aminobutyric acid (GABA), glucose, glutamate (Glu), glutamine (Gln), choline containing compounds (Cho) [glycerophosphocholine (GPC), phosphocoline (PCh)], 
glutathione (GSH), inositol containing compounds (Ino), lactate, $\mathrm{N}$-acetyl aspartate (NAA), Nacetyl aspartyl glutamate (NAAG), scylloinositol, taurine are measurable metabolites by a single voxel Point Resolved Spectroscopy Sequence (PRESS) in a 3 Tesla scanner (Buonocore and Maddock, 2015). In this study we could be able to quantify six metabolties including Glu, glutamate plus glutamine (Glx), NAA, Cho, Ino and Cr.

\section{Statistical Analysis}

Statistical analyses were performed with SPSS 21 (IBM, Armonk, NY, USA) software. Distribution characteristics of the data are assessed with Kolmogorov-Smirnov and ShapiroWilks' Tests. Independent samples $t$ Test or Mann-Whitney U Tests were performed for two independent group comparisons. Categorical variables were compared with chi-square test. Correlations were examined with Pearson's and Spearman's Correlation Tests. Univariate Analysis of Variance (ANOVA) and Analysis of Covariance (ANCOVA) was performed for three group comparisons for main effects. Chlorpromazine dose equivalents of antipsychotics, age, gender, age at onset, number of episodes, smoking status and alcohol consuming were available variables for covariate analysis. Interaction effects for grey matter, white matter and CSF contributions to the voxels were checked with interaction effect analysis in univariate ANOVA. Kruskal-Wallis Test was used for the comparison of non-Gaussian distributed variables. Post-hoc tests were Bonferroni or Tamhane Tests according to Levene's Homogeneity of Variance Test. Since 6 ANOVAs were performed for 6 metabolites (Glutamate, glutamate+glutamine, inositol containing compounds, creatine+phosphocreatine, choline containing compounds, n-Acetyl Aspartate) in the two hemispheres, a Bonferroni correction was applied to the $\mathrm{p}$ values $(6$ metabolites $\mathrm{X} 2$ hemispheres $=12 ; 0.05 / 12=0.0041$ ) and level for statistical significance was 0.0041 for metabolite level comparisons. For all other comparisons, cut-off for statistical significance was 0.05 . 
Figure 1. Voxel location (a) and representative spectrum (b)
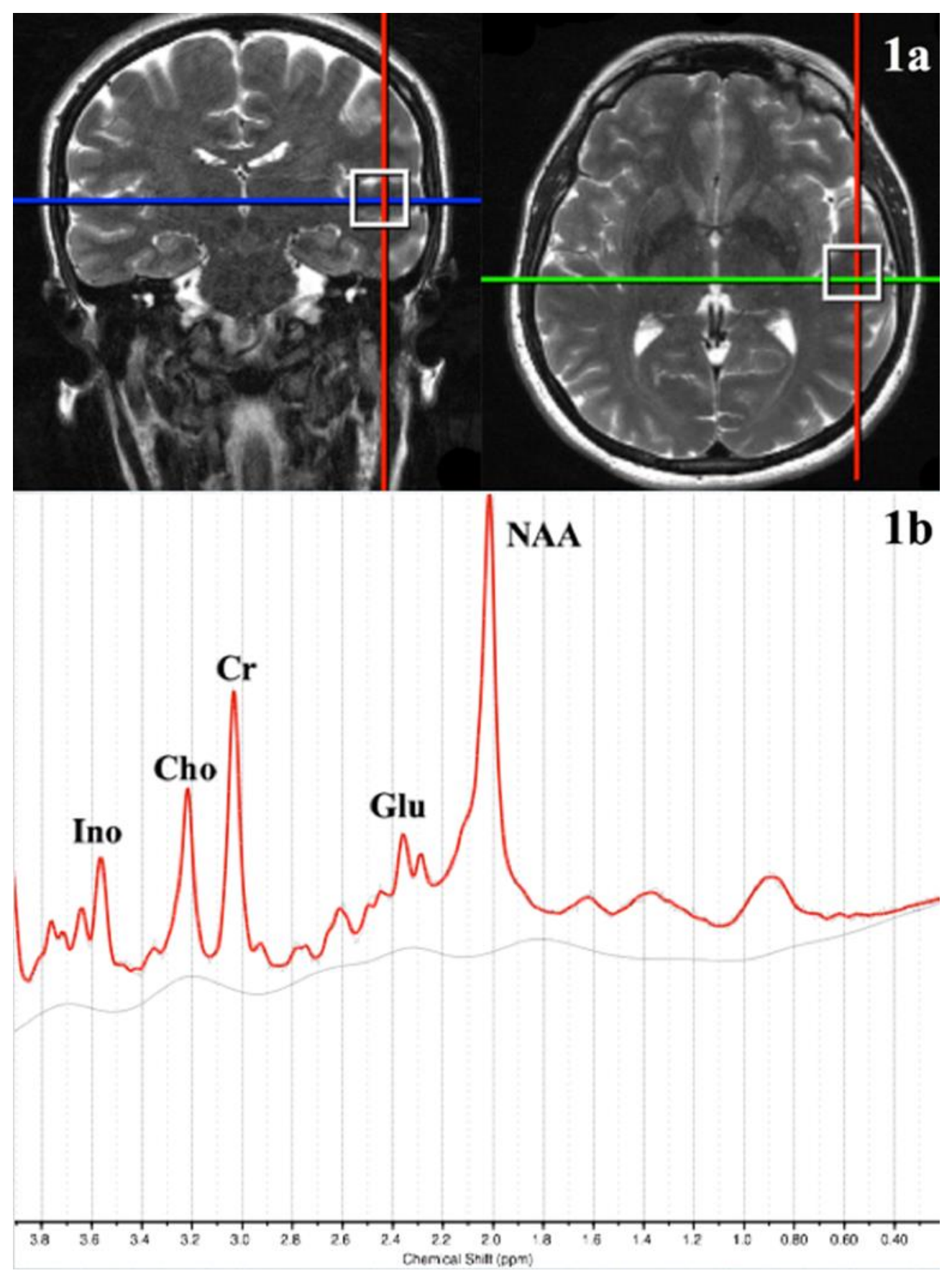

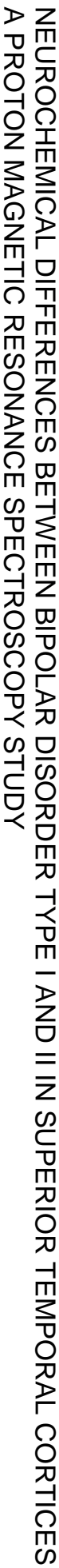

\section{Results}

Groups were similar in terms of age, gender, and education (Table 1). Smokers were more frequent in the patient groups $[\mathrm{F}=6.99, \mathrm{p}=0.030]$ and alcohol consumers were more frequent in the BD-II patient group $\left[\chi^{2}=9.69, \mathrm{p}=0.008\right]$. 
Table 1. Sociodemographic characteristics of the groups

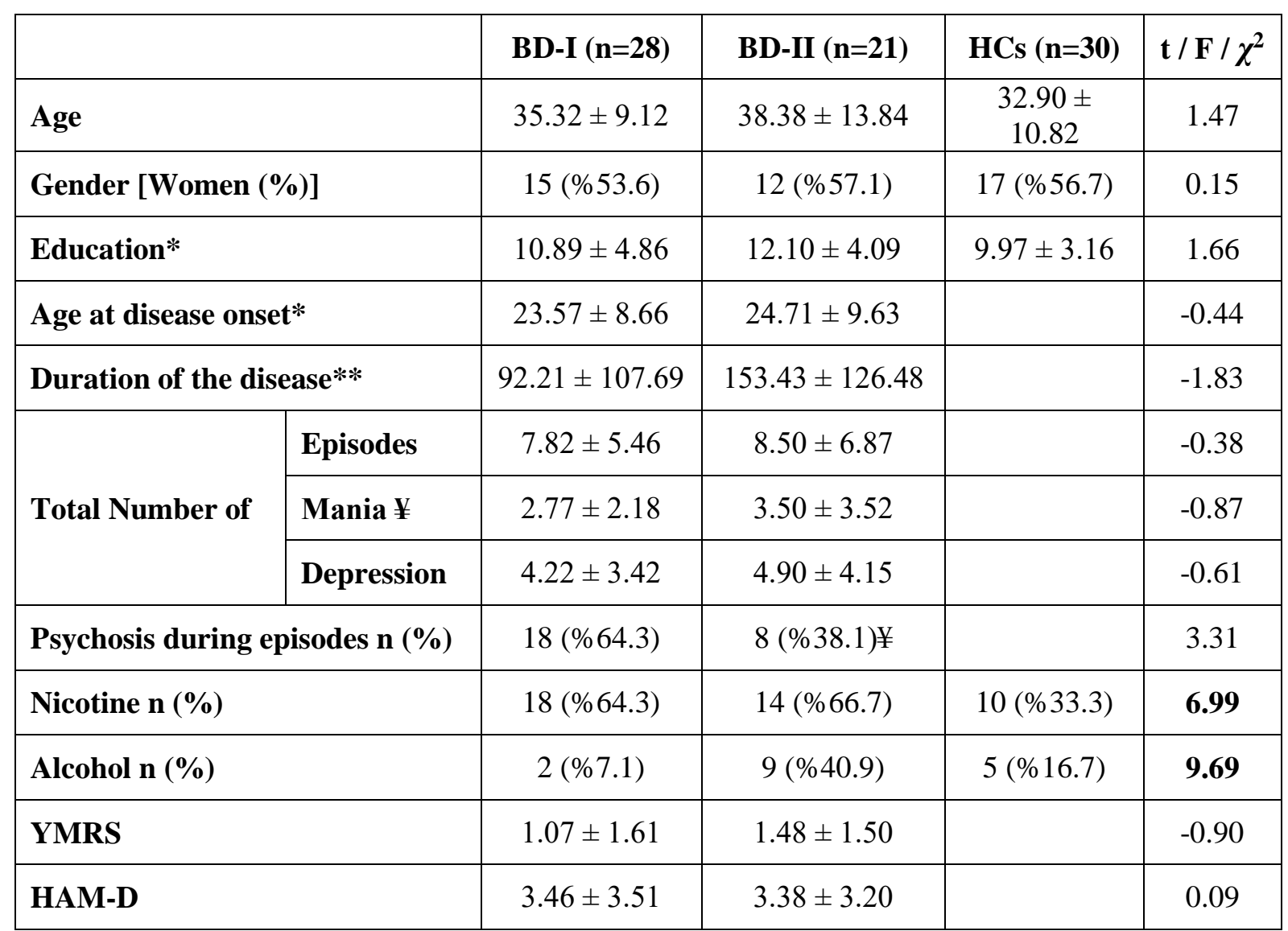

BD-I: Bipolar I Disorder, BD-II: Bipolar II Disorder, HCs: Healthy Controls, *Years, **Months, $¥$ Hypomania for patients with bipolar II disorder. YMRS: Young Mania Rating Scale, HAM-D: Hamilton Depression Rating Scale. Bold characters indicate statistical significance. ¥Psychotic symptoms were observed in depressive episodes of BD-II.

Comparison of the metabolite levels revealed that there were significant differences between groups in terms of glutamate [F=6.27, $\mathrm{p}=0.003$ ], glutamate + glutamine [F=6.08, $\mathrm{p}=0.004]$, inositol containing compounds (Ino) $[\mathrm{F}=9.25, \mathrm{p}<0.001]$, NAA $[\mathrm{F}=7.63, \mathrm{p}=0.001]$ and creatine + phosphocreatine $[\mathrm{F}=11.06, \mathrm{p}<0.001]$ in the left hemisphere (Table 2). Post-hoc comparisons showed that the BD-I disorder group had significantly lower metabolite levels in comparison to the BD-II and the $\mathrm{HC}$ groups (Table 2). There was no significant difference between the groups in terms of voxel segmentation data (gray / white matter and cerebrospinal fluid content, results are presented in Table 2). 
Table 2. ${ }^{1} \mathrm{H}-\mathrm{MRS}$ findings

\begin{tabular}{|c|c|c|c|c|c|c|c|c|}
\hline & & $\begin{array}{c}\text { BD-I } \\
(\mathrm{n}=28)\end{array}$ & $\begin{array}{l}\text { BD-II } \\
(\mathrm{n}=21)\end{array}$ & $\begin{array}{c}\text { HCs } \\
(n=30)\end{array}$ & $\mathbf{F}$ & $\mathbf{p}$ & $\begin{array}{c}\text { Partial } \\
\eta^{2}\end{array}$ & Post-hoc \\
\hline \multirow{9}{*}{ 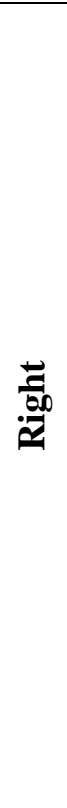 } & Glutamate & $\begin{array}{c}1.31 \pm \\
0.16\end{array}$ & $\begin{array}{c}1.46 \pm \\
0.24\end{array}$ & $\begin{array}{c}1.43 \pm \\
0.18\end{array}$ & 3.98 & 0.023 & 0.11 & \\
\hline & Glx & $\begin{array}{c}1.87 \pm \\
0.26\end{array}$ & $\begin{array}{c}1.98 \pm \\
0.26\end{array}$ & $\begin{array}{c}1.95 \pm \\
0.26\end{array}$ & 1.19 & 0.311 & 0.04 & \\
\hline & $\begin{array}{l}\text { Inositol } \\
\text { CC }\end{array}$ & $\begin{array}{c}0.92 \pm \\
0.15\end{array}$ & $\begin{array}{c}1.02 \pm \\
0.21\end{array}$ & $\begin{array}{c}1.06 \pm \\
0.09\end{array}$ & 5.65 & 0.005 & 0.25 & \\
\hline & $\begin{array}{l}\text { Choline } \\
\text { CC }\end{array}$ & $\begin{array}{c}0.29 \pm \\
0.05\end{array}$ & $\begin{array}{c}0.30 \pm \\
0.05\end{array}$ & $\begin{array}{c}0.28 \pm \\
0.06\end{array}$ & 1.04 & 0.358 & 0.03 & \\
\hline & NAA & $\begin{array}{c}1.59 \pm \\
0.18\end{array}$ & $\begin{array}{c}1.72 \pm \\
0.18\end{array}$ & $\begin{array}{c}1.74 \pm \\
0.20\end{array}$ & 4.94 & 0.010 & 0.13 & \\
\hline & $\mathrm{Cr}$ & $\begin{array}{c}1.12 \pm \\
0.14 \\
\end{array}$ & $\begin{array}{c}1.27 \pm \\
0.19 \\
\end{array}$ & $\begin{array}{c}1.18 \pm \\
0.18 \\
\end{array}$ & 4.38 & 0.016 & 0.11 & \\
\hline & $\begin{array}{l}\text { Gray } \\
\text { Matter* }\end{array}$ & $\begin{array}{c}56.83 \pm \\
4.78\end{array}$ & $\begin{array}{c}57.50 \pm \\
13.89\end{array}$ & $\begin{array}{c}58.00 \pm \\
4.87\end{array}$ & 0.14 & 0.870 & $<0.01$ & \\
\hline & $\begin{array}{l}\text { White } \\
\text { Matter* }\end{array}$ & $\begin{array}{c}28.91 \pm \\
8.09\end{array}$ & $\begin{array}{c}29.01 \pm \\
5.11\end{array}$ & $\begin{array}{c}30.58 \pm \\
6.47\end{array}$ & 0.51 & 0.604 & 0.01 & \\
\hline & $\mathrm{CSF}^{*}$ & $\begin{array}{c}13.58 \pm \\
7.20\end{array}$ & $\begin{array}{c}13.00 \pm \\
11.72\end{array}$ & $\begin{array}{c}10.74 \pm \\
5.49\end{array}$ & 0.93 & 0.401 & 0.02 & \\
\hline \multirow{9}{*}{ 氖 } & Glutamate & $\begin{array}{c}0.82 \pm \\
0.11 \\
\end{array}$ & $\begin{array}{c}0.99 \pm \\
0.25\end{array}$ & $\begin{array}{c}0.95 \pm \\
0.13 \\
\end{array}$ & 6.27 & 0.003 & 0.15 & $\begin{array}{c}\text { BD-I } \\
<\mathrm{HCs}, \mathrm{BD}-\mathrm{II}\end{array}$ \\
\hline & Glx & $\begin{array}{c}1.11 \pm \\
0.18\end{array}$ & $\begin{array}{c}1.36 \pm \\
0.37 \\
\end{array}$ & $\begin{array}{c}1.25 \pm \\
0.18 \\
\end{array}$ & 6.08 & 0.004 & 0.15 & $\begin{array}{c}\text { BD- } \\
\mathrm{I}<\mathrm{HCs}, \mathrm{BD}-\mathrm{II}\end{array}$ \\
\hline & $\begin{array}{l}\text { Inositol } \\
\text { CC }\end{array}$ & $\begin{array}{c}0.57 \pm \\
0.12\end{array}$ & $\begin{array}{c}0.72 \pm \\
0.17\end{array}$ & $\begin{array}{c}0.67 \pm \\
0.09\end{array}$ & 9.25 & $<0.001$ & 0.21 & $\begin{array}{c}\text { BD-I } \\
<\text { HCs,BD-II }\end{array}$ \\
\hline & $\begin{array}{l}\text { Choline } \\
\text { CC }\end{array}$ & $\begin{array}{c}0.17 \pm \\
0.03\end{array}$ & $\begin{array}{c}0.20 \pm \\
0.04\end{array}$ & $\begin{array}{c}0.19 \pm \\
0.03 \\
\end{array}$ & 4.18 & 0.019 & 0.11 & \\
\hline & NAA & $\begin{array}{c}1.02 \pm \\
0.13 \\
\end{array}$ & $\begin{array}{c}1.23 \pm \\
0.29 \\
\end{array}$ & $\begin{array}{c}1.17 \pm \\
0.13 \\
\end{array}$ & 7.63 & 0.001 & 0.18 & $\begin{array}{c}\text { BD-I } \\
<\text { HCs,BD-II } \\
\end{array}$ \\
\hline & $\mathrm{Cr}$ & $\begin{array}{c}0.71 \pm \\
0.11\end{array}$ & $\begin{array}{c}0.90 \pm \\
0.19\end{array}$ & $\begin{array}{c}0.81 \pm \\
0.10\end{array}$ & 11.06 & $<0.001$ & 0.23 & $\begin{array}{c}\text { BD-I } \\
<\mathrm{HCs}, \mathrm{BD}-\mathrm{II}\end{array}$ \\
\hline & $\begin{array}{l}\text { Gray } \\
\text { Matter* }\end{array}$ & $\begin{array}{c}57.03 \pm \\
4.42 \\
\end{array}$ & $\begin{array}{c}57.05 \pm \\
14.84 \\
\end{array}$ & $\begin{array}{c}59.78 \pm \\
5.73\end{array}$ & 0.85 & 0.432 & 0.22 & \\
\hline & $\begin{array}{l}\text { White } \\
\text { Matter* }\end{array}$ & $\begin{array}{c}31.06 \pm \\
9.46\end{array}$ & $\begin{array}{c}30.67 \pm \\
7.77\end{array}$ & $\begin{array}{c}30.51 \pm \\
6.72\end{array}$ & 0.04 & 0.966 & $<0.01$ & \\
\hline & $\mathrm{CSF}^{*}$ & $\begin{array}{c}10.25 \pm \\
7.48\end{array}$ & $\begin{array}{c}11.85 \pm \\
11.27\end{array}$ & $\begin{array}{c}9.38 \pm \\
4.56\end{array}$ & 0.59 & 0.556 & 0.02 & \\
\hline
\end{tabular}

All metabolite levels are in institutional units and rounded up (X 1000). Univariate ANOVA, Bonferroni or Tamhane Test were picked for post-hoc comparisons. Glx: Glutamate + Glutamine, NAA: N-AcetylAspartate, Inositol CC: Inositol containing compounds, Choline CC: Choline Containing Compounds, Cr: Creatine + Phosphocreatine, CSF: Cerebrospinal Fluid content. *Voxel Segmentation Data (percentage), Bold indicate statistical significance.

Correlation analysis was performed with Glu, Glx, NAA, Ino, Cr, age, age at onset, duration of the disease, number of episodes, serum lithium levels, serum valproate levels, chlorpromazine equivalent doses of antipsychotics, Young Mania Rating Scale and Hamilton Depression Rating Scale scores variables. There were significant correlations between right hemisphere N-Acetyl Aspartate (NAA) levels and age at disease onset $\left(\mathrm{r}^{2}=-0.58, \mathrm{p}=0.009\right)$, number of hypomanic 
episodes $\left(r^{2}=-0.51, p=0.032\right)$ in the BD-II group. ANCOVA showed that right hemisphere NAA levels were associated with the YMRS scores $[\mathrm{F}=5.36, \mathrm{p}=0.027]$, the number of life-time hypomanic episodes $[\mathrm{F}=8.69, \mathrm{p}=0.022]$, age of disease onset $[\mathrm{F}=5.86, \mathrm{p}=0.006]$ in the BD-II group. No other significant correlation was observed. Because we presented the BD-I group findings in the past, here we only present correlation analyses within the BD-II group. Medications are presented in Table 3.

Table 3. Medications of the patients

\begin{tabular}{|l|c|c|c|}
\hline & BD-I (n=28) & BD-II (n=21) & p \\
\hline Lithium & $10(\% 42.9)$ & $5(\% 23.8)$ & 0.752 \\
\hline Serum lithium levels (mEq/L) & $0.67 \pm 0.22$ & $0.54 \pm 0.15$ & 0.364 \\
\hline Valproate & $14(\% 50)$ & $6(\% 28.6)$ & 0.111 \\
\hline Serum valproate levels (mg/L) & $73.53 \pm 27.08$ & $61.50 \pm 25.76$ & 0.469 \\
\hline Antipsychotics & $14(\% 50)$ & $11(\% 52.4)$ & 0.549 \\
\hline CPZE & $297.71 \pm 246.93$ & $180.27 \pm 153.33$ & 0.120 \\
\hline Antidepressants* & $3(\% 10.7)$ & $5(\% 23.8)$ & 0.457 \\
\hline
\end{tabular}

*In combination with other medications. CPZE: Chlorpromazine equivalent doses of antipsychotics

Metabolite levels did not differ between patients on/off lithium, valproate ( $p>0.05$ for all). There were no significant differences between psychotic $(n=26)$ and non-psychotic $(n=23)$ patients independent of diagnostic subtypes ( $p>0.05$ for all metabolites in both hemispheres). Psychosis, alcohol or nicotine consumption did not show any significant impact on the metabolite levels ( $p>0.05$; Table 4 and Table 5).

Table 4. Comparison between psychotic and non-psychotic patients

\begin{tabular}{|l|c|c|c|c|}
\hline & $\begin{array}{c}\text { Patients with psychotic } \\
\text { episodes }(\mathbf{n}=\mathbf{2 6})\end{array}$ & $\begin{array}{c}\text { Patients without any psychotic } \\
\text { episodes }(\mathbf{n = 2 3})\end{array}$ & $\mathbf{t}$ & $\mathbf{p}$ \\
\hline Glu & $0.85 \pm 0.18$ & $0.95 \pm 0.22$ & 1.63 & 0.110 \\
\hline Glx & $1.18 \pm 0.26$ & $1.23 \pm 0.35$ & 0.97 & 0.337 \\
\hline NAA & $1.10 \pm 0.24$ & $1.13 \pm 0.25$ & 0.55 & 0.582 \\
\hline Ino & $0.62 \pm 0.15$ & $0.65 \pm 0.16$ & 0.64 & 0.523 \\
\hline Cr & $0.78 \pm 0.18$ & $0.81 \pm 0.17$ & 0.62 & 0.539 \\
\hline
\end{tabular}

Abbreviations are as written in unabbreviated form in Table 4. Psychotic and non-psychotic patients regardless of diagnostic subtypes are pooled in this analysis. 
Table 5. Covariate analysis

\begin{tabular}{|c|c|c|c|c|c|c|c|}
\hline & $\begin{array}{l}\text { BD-I } \\
(n=28)\end{array}$ & $\begin{array}{l}\text { BD-II } \\
(\mathrm{n}=21)\end{array}$ & $\begin{array}{l}\text { HCs } \\
(n=30)\end{array}$ & $\mathbf{F}$ & $\mathbf{p}$ & $\begin{array}{l}\text { Partial } \\
\eta^{2}\end{array}$ & Post-hoc \\
\hline Glu & $0.82 \pm 0.11$ & $\begin{array}{ll}0.99 & \pm \\
0.25 & \end{array}$ & $\begin{array}{ll}0.95 & \pm \\
0.13 & \end{array}$ & 6.27 & 0.003 & 0.15 & $\begin{array}{l}\begin{array}{l}\text { BD-I } \\
<\mathrm{HCs}, \mathrm{BD}-\mathrm{II}\end{array}\end{array}$ \\
\hline CPZE & & & & 3.45 & 0.093 & 0.26 & \\
\hline Age & & & & 0.15 & 0.707 & 0.02 & \\
\hline Age at onset & & & & 1.71 & 0.218 & 0.15 & \\
\hline Number of episodes & & & & 2.49 & 0.146 & 0.20 & \\
\hline Gender & & & & 4.70 & 0.012 & 0.125 & \\
\hline Smokers (n) & & & & 3.83 & 0.079 & 0.28 & \\
\hline $\begin{array}{r}\text { Alcohol consumers } \\
(\mathbf{n})\end{array}$ & & & & 0.18 & 0.684 & 0.02 & \\
\hline Glx & $1.11 \pm 0.18$ & $\begin{array}{ll} & 1.36 \\
0.37 & \end{array}$ & $\begin{array}{ll}1.25 & \pm \\
0.18 & \end{array}$ & 6.08 & 0.004 & 0.15 & $\begin{array}{l}\text { BD- } \\
\mathrm{I}<\mathrm{HCs}, \mathrm{BD}-\mathrm{II}\end{array}$ \\
\hline CPZE & & & & 2.70 & 0.131 & 0.21 & \\
\hline Age & & & & 0.10 & 0.754 & 0.01 & \\
\hline Age at onset & & & & 0.55 & 0.474 & 0.05 & \\
\hline Number of episodes & & & & 2.55 & 0.141 & 0.20 & \\
\hline Gender & & & & 2.02 & 0.186 & 0.17 & \\
\hline Smokers (n) & & & & 0.95 & 0.352 & 0.09 & \\
\hline $\begin{array}{r}\text { Alcohol consumers } \\
\text { (n) }\end{array}$ & & & & 0.77 & 0.401 & 0.07 & \\
\hline Inositol CC & $0.57 \pm 0.12$ & $\begin{array}{ll}0.72 & \pm \\
0.17 & \end{array}$ & $\begin{array}{ll}0.67 & \pm \\
0.09 & \\
\end{array}$ & 9.25 & $<0.001$ & 0.21 & $\begin{array}{l}\text { BD-I } \\
<\mathrm{HCs}, \mathrm{BD}-\mathrm{II}\end{array}$ \\
\hline CPZE & & & & 0.15 & 0.709 & 0.02 & \\
\hline Age & & & & 0.78 & 0.398 & 0.07 & \\
\hline Age at onset & & & & 1.29 & 0.283 & 0.11 & \\
\hline Number of episodes & & & & 0.05 & 0.835 & $<0.01$ & \\
\hline Gender & & & & 3.53 & 0.090 & 0.07 & \\
\hline Smokers (n) & & & & 0.59 & 0.461 & 0.06 & \\
\hline $\begin{array}{r}\text { Alcohol consumers } \\
\text { (n) }\end{array}$ & & & & 0.52 & 0.825 & 0.06 & \\
\hline NAA & $1.02 \pm 0.13$ & $\begin{array}{ll}1.23 & \pm \\
0.29 & \\
\end{array}$ & $\begin{array}{ll}1.17 & \pm \\
0.13 & \\
\end{array}$ & 7.63 & 0.001 & 0.18 & $\begin{array}{l}\text { BD-I } \\
<\mathrm{HCs}, \mathrm{BD}-\mathrm{II}\end{array}$ \\
\hline CPZE & & & & 0.01 & 0.921 & $<0.01$ & \\
\hline Age & & & & 0.40 & 0.544 & 0.04 & \\
\hline Age at onset & & & & 0.87 & 0.374 & 0.09 & \\
\hline Number of episodes & & & & 0.07 & 0.805 & $<0.01$ & \\
\hline Gender & & & & 4.62 & 0.060 & 0.34 & \\
\hline Smokers (n) & & & & 1.59 & 0.239 & 0.15 & \\
\hline $\begin{array}{r}\text { Alcohol consumers } \\
\text { (n) }\end{array}$ & & & & 0.25 & 0.627 & 0.03 & \\
\hline $\mathrm{Cr}$ & $0.71 \pm 0.11$ & $\begin{array}{ll}0.90 & \pm \\
0.19 & \\
\end{array}$ & $\begin{array}{ll}0.81 & \pm \\
0.10 & \\
\end{array}$ & 11.06 & $<0.001$ & 0.23 & $\begin{array}{l}\text { BD-I } \\
<\mathrm{HCs,BD-II}\end{array}$ \\
\hline CPZE & & & & 0.35 & 0.569 & 0.03 & \\
\hline Age & & & & 0.23 & 0.645 & 0.02 & \\
\hline Age at onset & & & & 0.82 & 0.387 & 0.07 & \\
\hline Number of episodes & & & & 0.16 & 0.690 & 0.02 & \\
\hline Gender & & & & 3.87 & 0.078 & 0.28 & \\
\hline Smokers (n) & & & & 0.71 & 0.419 & 0.07 & \\
\hline $\begin{array}{r}\text { Alcohol consumers } \\
(\text { (n) }\end{array}$ & & & & 0.09 & 0.776 & 0.01 & \\
\hline
\end{tabular}

Univariate ANCOVA. CPZE: Chlorpromazine equivalent doses of antipsychotics. Only metabolites which had significant differences between the groups are presented in this table. Glu: Glutamate, Glx: Glutamate and glutamine, Inositol CC: Inositol containing compounds, NAA: N-Acetyl Aspartate, Cr: Creatine and phosphocreatine 


\section{Discussion}

In this study metabolite levels in the superior temporal cortices differed between the BD-I and BD-II groups. The BD-I group had significantly lower metabolite levels in comparison to the BD-II group. Further analysis showed that clinical factors such as psychosis, number of episodes and nicotine or alcohol consumption did not have any impact on metabolite levels. HDRS scores had inverse relationship between NAA and $\mathrm{Cr}$ in both right and left hemispheres and Ino and HDRS had correlation in the right hemisphere in BD-I (Atagun et al., 2015). Whereas NAA levels were negatively correlated with age at onset, number of hypomanic episodes and YMRS scores in the BD-II group.

Glutamatergic neurotransmission is maintained by glutamate to glutamine cycle and glial cells serve for the cycle. This cycle is differentially altered in manic and depressive states (Yüksel and Öngür, 2010). Globally Glx is elevated (Dager et al., 2004), however Glu levels are not significantly elevated in bipolar disorder (Yüksel and Öngür, 2010; Atagun et al., 2015). Since Glx consist of glutamate plus glutamine, elevation of Glx is probably associated with increased glutamine levels. Quantification of glutamine is not possible with low magnetic field scanners, and thus there are not much studies in the literature. Glutamine levels in anterior cingulate cortex were found to be increased in bipolar disorder (Ongur et al., 2008). These findings may indicate that glutamate to glutamine cycle is disturbed in bipolar disorder. There may be a difference in glial physiopathology in BD-I and BD-II.

Inositol levels are found to be changed in bipolar disorder and mood stabilizers alter inositol levels by influencing phospho-inositol cycle (Silverstone and McGrath, 2009). However, the ${ }^{1} \mathrm{H}-\mathrm{MRS}$ literature regarding Ino levels is mixed (Silverstone and Mcgrath, 2009). Controlling for voxel locations, medications, field strength might be useful in future studies. NAA is a marker of mitochondrial function for oxidative metabolism and neuronal function/viability; decrease of NAA levels indicate neuronal dysfunction (Moffett et al., 2007). Cr metabolites are involved in energy metabolism by transporting high energy phosphorous groups; $\mathrm{Cr}$ compounds are highly diffusible and are considered to be stable throughout the brain. However, it has been determined that $\mathrm{Cr}$ is not stable in bipolar disorder and schizophrenia (Ongur et al., 2009). Thus, the use of $\mathrm{Cr}$ as an internal reference might be erroneous in bipolar disorder and schizophrenia. Correlation analysis revealed differences between BD-I and BD-II. NAA levels are negatively correlated with number of hypomanic episodes and age at disease onset in BD- 
II. Whereas in BD-I HDRS scores were negatively correlated with Ino and NAA (reported in Atagun et al., 2015).

Small sample size and being a cross-sectional study are major limitations of this study. Furthermore, patients were on various psychotropic medications and medications are known to alter the metabolites measured by ${ }^{1} \mathrm{H}-\mathrm{MRS}$ (Moore et al., 2000; Moore et al., 2007). Because of its anatomical adjacency to cranium, $\mathrm{B}_{0}$ and $\mathrm{B}_{1}$ inhomogeneity are high in temporal lobe (Juchem and de Graff, 2016). $\mathrm{B}_{0}$ shimming was performed, however $\mathrm{B}_{1}$ shimming was not available in this study.

To conclude, levels of metabolites are decreased in BD-I in comparison to BD-II and healthy controls in this study. These findings may indicate that neuronal dysfunction and metabolic decline in the left (dominant) hemisphere superior temporal lobe is specific to BD-I. Although further studies are needed, it can be postulated that pathology of the left hemispheric superior temporal lobe might be more severe in BD-I in comparison to BD-II. Our findings indicate that the left hemispheric superior temporal lobe metabolite levels in BD-I are declined similar to psychotic spectrum disorders rather than BD-II. Further studies comparing manic and hypomanic episodes of BD-I and BD-II may reveal metabolic differences between the subtypes.

\section{References}

Ambrosi, E., Rossi-Espagnet, M.C., Kotzalidis, G.D., Comparelli, A., Del Casale, A., Carducci, F., Romano, A., Manfredi, G., Tatarelli, R., Bozzao, A., Girardi, P., 2013. Structural brain alterations in bipolar disorder II: a combined voxel-based morphometry (VBM) and diffusion tensor imaging (DTI) study. J. Affect. Disord. 150, 610-615.

Atagün, M.İ., Şıkoğlu, E.M., Can, S.S., Karakaş-Uğurlu, G., Ulusoy-Kaymak, S., Çayköylü, A., Algın, O., Phillips, M.L., Moore, C.M., Öngür, D., 2015. Investigation of Heschl's gyrus and planum temporale in patients with schizophrenia and bipolar disorder: A proton magnetic resonance spectroscopy study. Schizophr. Res. 161, 202-209.
Atagün, M.İ., Şıkoğlu, E.M., Soykan, Ç., Can, S.S., Ulusoy-Kaymak, S., Çayköylü, A., Algın, O., Phillips, M.L., Öngür, D., Moore, C.M., 2017. Perisylvian GABA levels in schizophrenia and bipolar disorder. Neurosci. Lett. 637, 70-74.

Bora, E., Yücel, M., Pantelis, C., Berk, M., 2011. Meta-analytic review of neurocognition in bipolar II disorder. Acta Psychiatr. Scand. 123, 165-174.

Dager, S.R., Friedman, S.D., Parow, A., Demopulos, C., Stoll, A.L., Lyoo, I.K., Dunner, D.L., Renshaw, P.F., 2004. Brain metabolic alterations in medication-free patients with bipolar disorder. Arch. Gen. Psychiatry 61, 450-458.

First, M.D., Gibbon, M., Spitzer, R.L., Gibbon, M., Williams, J.B.W., 1996. User's Guide 
for the Structured Interview for DSM-IV Axis I Disorders Research Version (SCID-I, version 2.0). Biometrics Research, New York.

Hamakawa, H., Kato, T., Murashita, J., Kato, N., 1998. Quantitative proton magnetic resonance spectroscopy of the basal ganglia in patients with affective disorders. Eur. Arch. Psychiatr. Clin. Neurosci. 248, 53-58.

Hamilton, M., 1960. A rating scale for depression. J. Neurol. Neurosurg. Psychiatry $23,56-62$.

Hanford, L.C., Nazarov, A., Hall, G.B., Sassi, R.B., 2016. Cortical thickness in bipolar disorder: a systematic review. Bipolar Disord. 18, 4-18.

Hauser, P., Matochik, J., Altshuler, L.L., Denicoff, K.D., Conrad, A., Li, X., Post., R.M., 2000. MRI-based measurements of temporal lobe and ventricular structures in patients with bipolar I and bipolar II disorders. J. Affect. Disord. 60, 25-32.

Juchem, C., de Graaf, R.A., 2016. B0 magnetic field homogeneity and shimming for in vivo magnetic resonance spectroscopy. Anal Biochem. 529, 17-29.

Kato, T., Hamakawa, H., Shioiri, T., Murashita, J., Takahashi, Y., Takahashi, S., Inubushi, T., 1996. Choline-containing compounds detected by proton magnetic resonance spectroscopy in the basal ganglia in bipolar disorder. J. Psychiatry Neurosci. 21, 248254.

Lee, S.Y., Chen, S.L., Chen, S.H., Huang, S.Y., Tzeng, N.S., Chang, Y.H., Wang, C.L., Lee, I.H., Yeh, T.L., Yang, Y.K., Lu, R.B., 2011. The COMT and DRD3 genes interacted in bipolar I but not bipolar II disorder. World J. Biol. Psychiatry 12, 385-391.

Lee, S.Y., Chen, S.L., Chang, Y.H., Chu, C.H., Huang, S.Y., Tzeng, N.S., Wang, C.L., Lin,
S.H., Lee, I.H., Yeh, T.L., Yang, Y.K., Lu R.B., 2012. The ALDH2 and 5-HT2A genes interacted in bipolar-I but not bipolar-II disorder. Prog. Neuropsychopharmacol. Biol. Psychiatry 38, 247-251.

Li, C.T., Hsieh, J.C., Wang, S.J., Yang, B.H., Bai, Y.M., Lin, W.C., Lan, C.C., Su, T.P., 2012. Differential relations between frontolimbic metabolism and executive function in patients with remitted bipolar I and bipolar II disorder. Bipolar Disord. 14, 831-842.

McCarley, R.W., Nakamura, M., Shenton, M.E., Salisbury, D.F., 2008. Combining ERP and structural MRI information in first episode schizophrenia and bipolar disorder. Clin. EEG Neurosci. 39(2), 57-60.

McGrath, B.M., Wessels, P.H., Bell, E.C., Ulrich, M., Silverstone, P.H., 2014. Neurobiological findings in bipolar II disorder compared with findings in bipolar I disorder. Can. J. Psychiatry 49, 794-801.

McNamara, R.K., Rider, T., Jandacek, R., Tso, P., 2014. Abnormal Fatty Acid Pattern in the Superior Temporal Gyrus Distinguishes Bipolar Disorder from Major Depression and Schizophrenia and Resembles Multiple Sclerosis. Psychiatry Res. 215(3), 560-567.

Moffett, J.R., Ross, B., Arun, P., Madhavarao, C.N., Namboodiri, A.M., 2007. NAcetylaspartate in the CNS: from neurodiagnostics to neurobiology. Prog. Neurobiol. 81, 89-131.

Moore, C.M., Biederman, J., Wozniak, J., Mick, E., Aleardi, M., Wardrop, M, Dougherty, M., Harpold, T., Hammerness, P., Randall, E., Lyoo, I.K., Renshaw, P.F., 2007. Mania, glutamate/glutamine and risperidone in pediatric bipolar disorder: a proton magnetic resonance spectroscopy study of the anterior cingulate cortex. J. Affect. Disord. 99, 1925. 
Moore G.J., Bebchuk, J.M., Hasanat, K., Chen, G., Seraji-Bozorgzad, N., Wilds, I.B., Faulk, M.W., Koch, S., Glitz, D.A., Jolkovsky, L., Manji, H.K., 2000. Lithium increases Nacetyl-aspartate in the human brain: in vivo evidence in support of bcl-2's neurotrophic effects? Biol. Psychiatry 48, 1-8.

Nudmamud, S., Reynolds, L.M., Reynolds, G.P., 2003. N-acetylaspartate and NAcetylaspartylglutamate deficits in superior temporal cortex in schizophrenia and bipolar disorder: a postmortem study. Biol. Psychiatry 53, 1138-1141.

Ongur, D., Jensen, J.E., Prescot, A.P., Stork, C., Lundy, M., Cohen, B.M., Renshaw, P.F., 2008. Abnormal glutamatergic neurotransmission and neuronal-glial interactions in acute mania. Biol. Psychiatry 64 (8), 718-726.

Ongur, D., Prescot, A.P., Jensen, J.E., Cohen, B.M., Renshaw, P.F., 2009. Creatine abnormalities in schizophrenia and bipolar disorder. Psychiatry Res. 172 (1), 44-48.

Özerdem, A., Güntekin, B., Atagün, I., Turp, B., Başar, E., 2011. Reduced long distance gamma (28-48 Hz) coherence in euthymic patients with bipolar disorder. J. Affect. Disord. 132(3), 325-332.

Parker, G., Fletcher, K., 2014. Differentiating bipolar I and II disorders and the likely contribution of DSM- 5 classification to their cleavage. J. Affect. Disord. 152-154, 57-64.

Phillips, M.L., Kupfer, D.J., 2013. Bipolar disorder diagnosis: challenges and future directions. Lancet 381, 1663-1671.

Provencher, S.W., 2001. Automatic quantitation of localized in vivo $1 \mathrm{H}$ spectra with LCModel. NMR Biomed. 14, 260-264.
Silverstone, P.H., Asghar, S.J., O’Donnell, T., Ulrich, M., Hanstock, C.C., 2004. Lithium and valproate protect against dextroamphetamine induced brain choline concentration changes in bipolar disorder patients. World. J. Biol. Psychiatry 5, 38-44.

Silverstone, P.H., McGrath, B.M., 2009. Lithium and valproate and their possible effects on themyo-inositol second messenger system in healthy volunteers and bipolar patients. Int. Rev. Psychiatry 21(4), 414-423.

Uemura, T., Green, M., Corson, T.W., Perova, T., Li, P.P., Warsh, J.J., 2011. Bcl-2 SNP rs956572 associates with disrupted intracellular calcium homeostasis in bipolar I disorder. Bipolar Disord. 13, 41-51.

Winsberg, M.E., Sachs, N., Tate, D.L., Adalsteinsson, E., Spielman, D., Ketter, T.A., 2000. Decreased dorsolateral prefrontal $\mathrm{N}$-acetylaspartate in bipolar disorder. Biol. Psychiatry 47, 475-481.

Young, R.C., Biggs, J.T., Ziegler, V.E., Meyer, D.A., 1978. A rating scale for mania: reliability, validity and sensitivity. Br. J. Psychiatry 133, 429-435.

Yüksel, C., Öngür, D., 2010. Magnetic resonance spectroscopy studies of glutamate-related abnormalities in mood disorders. Biol. Psychiatry 68(9), 785-794.

Yumru, M., Savas, H.A., Kalenderoglu, A., Bulut, M., Celik, H., Erel, O., 2009. Oxidative imbalance in bipolar disorder subtypes: A comparative study. Prog. Neuropsychopharmacol. Biol. Psychiatry 33, 1070-1074. 


\section{CHAPTER 8}

\section{Perisylvian GABA levels in schizophrenia and bipolar disorder}

Murat IIIhan ATAGÜN, Elif Muazzez ŞIKOĞLU, Çağlar SOYKAN, Serdar Süleyman CAN, Semra ULUSOY-KAYMAK, Ali ÇAYKÖYLÜ, Oktay ALGIN, Mary Louise PHILIPS, Dost ÖNGÜR, Constance Mary MOORE

Neuroscience Letters 2017; 637: 70-74 


\section{Abstract}

The aim of this study is to measure GABA levels of perisylvian cortices in schizophrenia and bipolar disorder patients, using proton magnetic resonance spectroscopy $\left({ }^{1} \mathrm{H}-\mathrm{MRS}\right)$. Patients with schizophrenia ( $\mathrm{n}=25)$, bipolar I disorder (BD-I; $\mathrm{n}=28)$ and bipolar II disorder (BD-II; $\mathrm{n}=20)$ were compared with healthy controls $(\mathrm{n}=30) .{ }^{1} \mathrm{H}-\mathrm{MRS}$ data was acquired using a Siemens 3 Tesla whole body scanner to quantify right and left perisylvian structures' (including superior temporal lobes) GABA levels. Right perisylvian GABA values differed significantly between groups $\left[\chi^{2}=9.62\right.$, df: $\left.3, p=0.022\right]$. GABA levels were significantly higher in the schizophrenia group compared with the healthy control group $(\mathrm{p}=0.002)$. Furthermore, Chlorpromazine equivalent doses of antipsychotics correlated with right hemisphere GABA levels $\left(\mathrm{r}^{2}=0.68, \mathrm{p}=0.006, \mathrm{n}=33\right)$. GABA levels are elevated in the right hemisphere in patients with schizophrenia in comparison to bipolar disorder and healthy controls. The balance between excitatory and inhibitory controls over the cortical circuits may have direct relationship with GABAergic functions in auditory cortices. In addition, GABA levels may be altered by brain regions of interest, psychotropic medications, and clinical stage in schizophrenia and bipolar disorder.

Key words: Schizophrenia, Bipolar Disorder, GABA, Magnetic Resonance Spectroscopy, Auditory Cortex 


\section{Introduction}

Several lines of evidence have converged that as an inhibitory neurotransmitter, Gamma-Amino Butyric Acid (GABA) neurotransmission serves for network integrity by facilitating neural synchronization in the brain (Bartos et al., 2007). Postmortem studies have shown abnormalities in GABAergic cells (Benes and Berretta, 2001; Ishikawa et al., 2005; Mizukami et al., 2000) and these findings suggested that disturbances in the early phases of brain development may lead to abnormalities of GABAergic neurotransmission possibly causing dysregulation in the inhibitory and excitatory neurotransmission in cortical circuitries (Benes and Berretta, 2001). Disturbed GABAergic neurotransmission may lead to abnormalities in integrative brain functions and cognitive dysfunction (Gonzalez-Burgos et al., 2011).

Irregularities in GABA neurotransmission have critical roles in the pathophysiology of schizophrenia and bipolar disorder (Benes and Berretta, 2001). Altered RNA, protein and neurochemical markers of interneurons (Torrey et al., 2005), decreased number (Wang et al., 2011) and disturbed maturation of GABAergic cells (Gandal et al., 2012) have indicated GABAergic dysfunction in schizophrenia and bipolar disorder. Measurements of GABA levels using proton magnetic resonance spectroscopy $\left({ }^{1} \mathrm{H}-\mathrm{MRS}\right)$ have reported altered GABA levels in schizophrenia (Öngür et al., 2010; Wijtenburg et al., 2015) and bipolar disorder (Bhagwagar et al., 2007; Brady et al., 2013; Kaufman et al. 2009). However, the findings are inconsistent possibly due to a number of reasons including different MRS methods, variability between brain regions of interest, medication effects and clinical course [10]. Most studies have focused on frontal, prefrontal, parietal or occipital cortices, medicated patients and clinically remitted patients, and all of these factors, including brain regions of interest, psychotropic medications, and clinical stage, may have significant effects on GABA levels. 
The auditory cortices have a long and delicate developmental trajectory (Moore, 2002), which is vulnerable to the pathophysiology of schizophrenia and bipolar disorder (Mccarley et al., 2002). Since auditory hallucinations are one of the most frequent symptoms of schizophrenia and abnormalities of the auditory cortices are associated with hallucinations (Jardri et al., 2011), auditory cortices are among the most relevant brain regions in schizophrenia. In a recent ${ }^{1} \mathrm{H}-$ MRS study, we have detected metabolic abnormalities within the left hemisphere superior temporal lobe in both schizophrenia and bipolar disorder (Atagun et al., 2015). Neural synchronization deficits with auditory tasks may indicate GABAergic abnormalities in auditory cortices in bipolar disorder and schizophrenia (Atagun, 2016). In addition, a recent ${ }^{1} \mathrm{H}-\mathrm{MRS}$ study report decreased GABA levels in the left perisylvian cortices in autism, this is consistent with the theory of excitatory-inhibitory balance dysregulation in autism spectrum disorders (Rojas et al., 2019).

In this study, we aimed to investigate GABA levels within the auditory belt and parabelt regions located around the Sylvian (Lateral) Fissure, which host primary and association auditory cortices. To our knowledge, this is the first study that measure GABA levels at the perisylvian structures in schizophrenia and bipolar disorder. Since there are abnormalities in excitatory neurotransmission and GABAergic cells (Bernstein et al., 2015), we hypothesized that GABA levels might be altered in schizophrenia and bipolar disorder.

\section{Materials and methods}

\subsection{Participants}

The local Ethical Committee of Ankara Yıldırım Beyazıt University Medical School has approved the study. All participants provided written consent after the study procedures were fully explained. Remitted patients with schizophrenia (n=25), bipolar I disorder (BD-I) (n=28), bipolar II disorder (BD-II) $(n=20)$ and a healthy control group (HC) $(n=30)$ were enrolled. Socio-demographic features are presented in Table 1. Exclusion criteria were history of brain 
damage or surgery, MR incompatible metallic implants or prosthesis, systemic diseases, hearing disability, lifetime history of psychiatric comorbidity and/or substance abuse. All medications were allowed except benzodiazepines. The following clinical evaluations were administered by MIA: Structured Clinical Interview according for the DSM-IV (SCID-I) (First et al., 1996), Young Mania Rating Scale (YMRS) (Young et al., 1978), Hamilton Depression Rating Scale (HDRS) (Hamilton, 1960), Scale for the Assessment of Positive Symptoms (SAPS) (Andreasen, 1984), Scale for the Assessment of Negative Symptoms (SANS) (Andreasen, 1983) and Brief Psychiatric Rating Scale (BPRS) (Overall, 1962). All subjects completed an MR data acquisition session immediately following the clinical evaluations.

\subsection{Magnetic Resonance Imaging Data acquisition}

Data were acquired on a 3.0 Tesla Siemens MAGNETOM TIM Trio whole-body MR system (Siemens, Erlangen, Germany) with a thirty two-channel phased-array head coil at the UMRAM National Magnetic Resonance Research Center, Ankara, Turkey. T1-weighted anatomical MRI (MPRAGE sequence, 256 X 256 voxels, TR: 2000 msec, TE: 3.02 msec, FOV read: 215 , FOV phase: 100, slice thickness: 0.84, 192 slices) were collected for diagnostic and localization purposes. Proton Magnetic Resonance Spectroscopy $\left({ }^{1} \mathrm{H}\right.$ MRS) data was acquired using the single voxel Point REsolved Spectroscopy Sequence (PRESS) $(\mathrm{TE}=30 \mathrm{msec}, \mathrm{TR}=$ $2000 \mathrm{msec}$ ) to quantify brain creatine (Cr) levels and MEscher-GArwood Point-REsolved Spectroscopy Sequence (MEGAPRESS) (Mescher et al., 1998; Waddell et al., 2007) (TE = 68 msec, $\mathrm{TR}=2000 \mathrm{msec}$ ) to quantify brain GABA levels. Voxels (PRESS: $20 \mathrm{~mm} \mathrm{X} 20 \mathrm{~mm} \mathrm{X}$ $20 \mathrm{~mm}$; MEGAPRESS: $30 \mathrm{~mm}$ X $30 \mathrm{~mm} \mathrm{X} 20 \mathrm{~mm}$ ) were placed in the structures around Sylvian Fissure including superior temporal lobe and inferior parietal lobe.

\subsection{Magnetic Resonance Imaging Data analysis}

The proton spectra were fit using LCModel (Version 6.3.0) to quantify the creatine levels (Provencher, 1993; Provencher, 2001) and GANNET software to quantify the GABA-to- 
creatine ratio (GABA/Cr) (Dydak et al., 2010; Edden et al., 2007; Edden et al., 2013; Zhu et al., 2011; Sikoglu et al., 2015; Cochran et al., 2015). The structural T1-weighted images were segmented using SPM8 [Statistical Parameter Mapping- Welcome Department of Imaging Neuroscience, London, UK; (http://www.fil.ion.ucl.ac.uk/spm/software/spm8/)] to determine the gray matter, white matter and CSF contributions to the voxel of interest. Absolute Cr values (Jardri et al., 2011) were corrected for voxel tissue content and then multiplied with the GABAto-Cr to determine the absolute GABA levels (Chowdury et al., 2014).

\subsection{Statistical Analysis}

Statistical analyses were performed using SPSS 22.0 software (Chicago, Illinois, USA). Outlier analysis was conducted and GABA values two standard deviations away from the mean of the corresponding groups were eliminated from further analysis. Chi-square test was used for the comparison of categorical variables. Shapiro-Wilk's tests for normality were performed for continuous variables. Two tailed independent samples t-test or Mann-Whitney U test were used for comparisons between independent groups. Group comparisons including more than two groups were performed by Univariate ANOVA or Kruskal-Wallis tests. Mann-Whitney U tests were performed for post-hoc comparisons after Kruskal-Wallis test. Since we had 4 groups and performed 6 Mann-Whitney $\mathrm{U}$ tests for posthoc comparisons between groups, we determined significance level as $0.0083(0.05 / 6=0.0083)$ according to Bonferroni correction. In addition, Pearson's correlation analysis was performed to determine the relationship between GABA levels and clinical assessments.

\section{Results}

The demographic and clinical characteristics of the sample are listed in Table 1. There were no significant demographic differences in sociodemographic variables. All patients were clinically stable. However, schizophrenia patients scored significantly higher than the bipolar disorder groups on BPRS $(\mathrm{F}(2,63)=21.76, \mathrm{p}<0.0001)$. 
Table 1. Clinical characteristics of the participants.

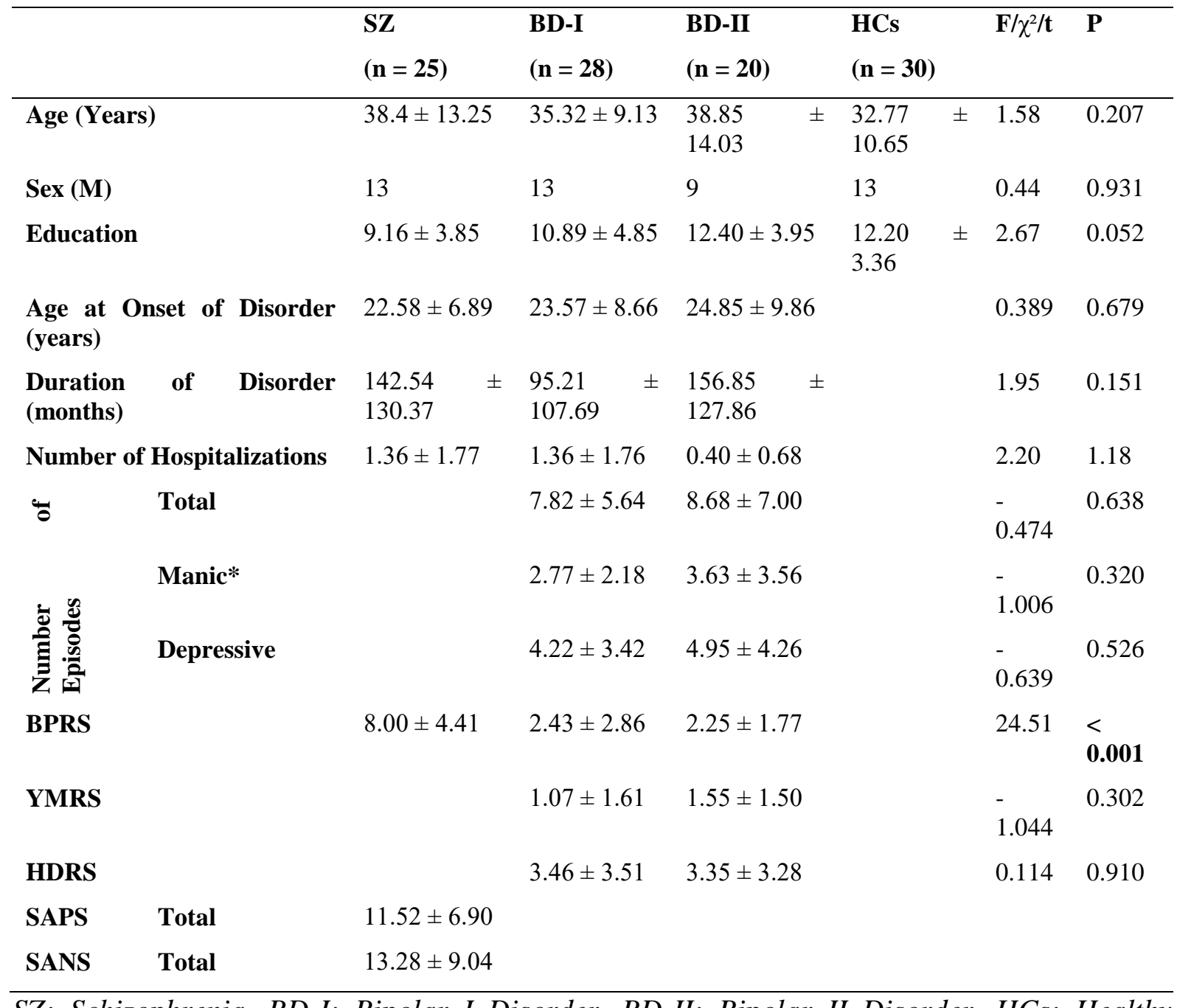

SZ: Schizophrenia, BD-I: Bipolar I Disorder, BD-II: Bipolar II Disorder, HCs: Healthy Controls, BPRS: Brief Psychiatric Rating Scale, HDRS: Hamilton Depression Rating Scale, SANS: Schedule for the Assessment of Negative Symptoms, SAPS: Schedule for the Assessment of Positive Symptoms, YMRS: Young Mania Rating Scale. * Hypomania for the Bipolar II Disorder.

GABA levels at the right hemisphere significantly differed between the groups $\left[\chi^{2}=9.62\right.$, df: 3 , $\mathrm{p}=0.022]$ (Table 2). Posthoc comparisons revealed that GABA levels in the schizophrenia group were significantly higher than the BD-I ( $\mathrm{p}=0.02)$, BD-II $(\mathrm{p}=0.02)$ and $\mathrm{HC}(\mathrm{p}=0.002, \mathrm{Z}=-3.08)$ groups (Figure 1). Difference between the groups was significant only between schizophrenia and $\mathrm{HC}$ after Bonferroni correction $(\mathrm{p}=0.002)$. There were no significant differences in the left hemisphere GABA levels between the groups $\left[\chi^{2}=1.63\right.$, df: 3 , $\left.p=0.652\right]$ (Table 2). GABA levels did not differ between the hemispheres within each group ( $p>0.05)$. 
Figure 1. GABA levels in groups. There was significant difference between groups at right hemisphere. Schizophrenia group had significantly higher GABA levels in comparison to healthy controls. There was no significant difference between groups at left hemisphere. BD-I: Bipolar I Disorder, BD-II: Bipolar II Disorder, SZ: Schizophrenia, HC: Healthy Controls. GABA levels are in international units (IU).
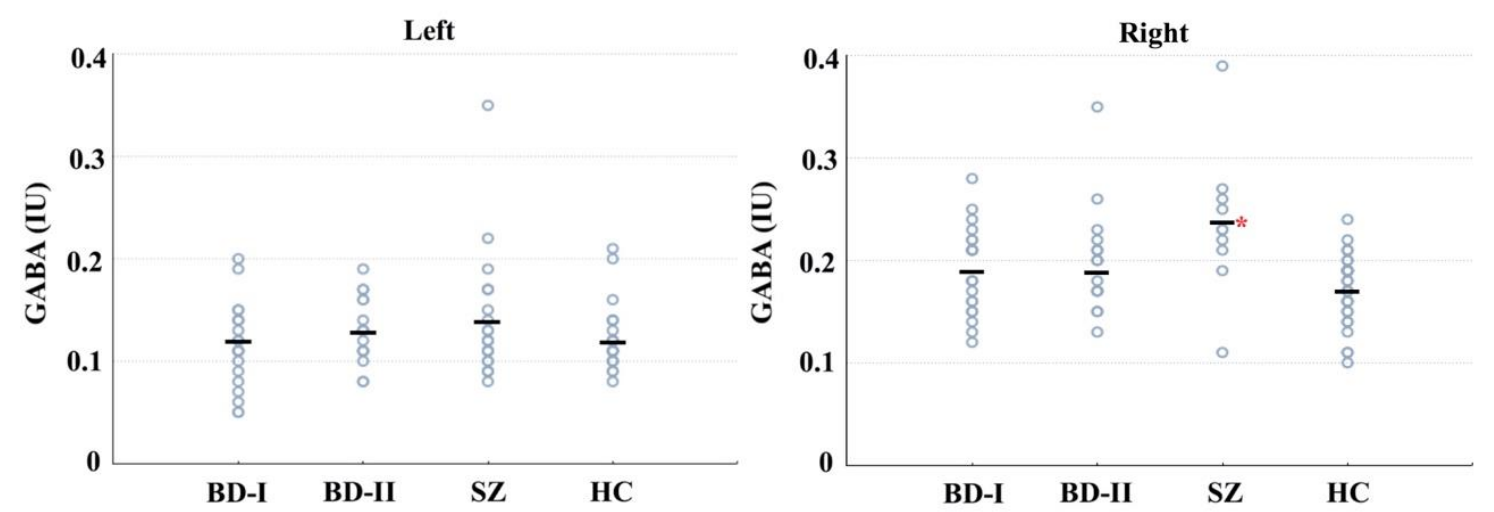

Table 2. GABA concentrations.

\begin{tabular}{lllllll}
\hline \multicolumn{2}{c}{ SZ } & BD-I & BD-II & HCs & $\chi^{2}$ (df) & P \\
\hline Left GABA & $0.12(0.11-0.17)$ & $0.11(0.09-0.14)$ & $0.13(0.11-0.16)$ & $0.12(0.10-0.14)$ & $1.63(3)$ & 0.652 \\
& & & & & & \\
Right GABA & $0.23(0.20-0.27)$ & $0.18(0.16-0.22)$ & $0.18(0.15-0.21)$ & $0.18(0.15-0.20)$ & $9.62(3)$ & $\mathbf{0 . 0 2 2}$
\end{tabular}

Kruskal-Wallis Test. Median (25-75 percentiles) values are reported. SZ: Schizophrenia, BD-

I: Bipolar I Disorder, BD-II: Bipolar II Disorder, HCs: Healthy Controls.

Patients with schizophrenia were on significantly more atypical anti-psychotics than the BD-I or BD-II groups $\left(\chi^{2}(2,73)=8.874, \mathrm{p}<0.012\right)$ (Table 3$)$. Chlorpromazine equivalents of antipsychotic doses were correlated positively with right hemisphere GABA levels $\left(r^{2}=0.68\right.$, $\mathrm{p}=0.006, \mathrm{n}=33$ : schizophrenia, BD-I and BD-II groups). Serum valproate levels correlated positively with left hemisphere GABA levels $\left(\mathrm{r}^{2}=0.8, \mathrm{p}=0.016, \mathrm{n}=14\right.$ : BD-I and BD-II groups). 
Table 3.Medication statuses of the patient groups.

\begin{tabular}{|c|c|c|c|c|c|}
\hline & $\begin{array}{l}\mathrm{SZ} \\
(\mathrm{n}=25)\end{array}$ & $\begin{array}{l}\text { BD-I } \\
(n=28)\end{array}$ & $\begin{array}{l}\text { BD-II } \\
(n=20)\end{array}$ & $\chi^{2} / Z$ & $\mathbf{P}$ \\
\hline $\begin{array}{l}\text { Atypical } \\
\text { Antipsychotics (n) }\end{array}$ & 22 & 14 & 12 & 8.87 & 0.012 \\
\hline $\begin{array}{l}\text { Chlorpromazine } \\
\text { Equivalent (mg) }\end{array}$ & $\begin{array}{l}225 \quad(145.75 \\
400)\end{array}$ & $267(133-400)$ & $150(50-267)$ & $\begin{array}{l}6.39 \\
(2)\end{array}$ & 0.041 \\
\hline Lithium (n) & 0 & 11 & 10 & 0.54 & 0.461 \\
\hline $\begin{array}{l}\text { Serum Lithium Levels } \\
(\mathrm{mEq} / \mathrm{L})\end{array}$ & & $\begin{array}{lll}0.70 & (0.45 \quad- \\
0.85) & & \end{array}$ & $\begin{array}{ll}0.70 & (0.45 \\
0.85) & \end{array}$ & $\begin{array}{ll}- & -0.47\end{array}$ & 0.658 \\
\hline Valproate (n) & 0 & 11 & 7 & -0.86 & 0.650 \\
\hline $\begin{array}{l}\text { Serum Valproate } \\
\text { Levels }(\mathrm{mcg} / \mathrm{ml})\end{array}$ & & $72.2(56-99.35)$ & $\begin{array}{l}57.3 \quad(44.8 \\
83.6)\end{array}$ & -0.91 & 0.386 \\
\hline
\end{tabular}

There was a correlation between left hemisphere GABA levels and the alogia subscale of the SANS $\left(\mathrm{r}^{2}=0.8, \mathrm{p}<0.05, \mathrm{n}=10\right)$. There was no significant correlation between GABA levels and YMRS $\left(\mathrm{r}^{2}=0.48, \mathrm{p}=0.158, \mathrm{n}=39\right)$ however, there was a trend for a negative correlation with HDRS $\left(r^{2}=0.53, p=0.08, n=39\right)$ in the bipolar disorder groups.

\section{Discussion}

GABA levels in the right perisylvian structures were higher in schizophrenia patients in comparison to bipolar disorder and healthy control groups. There was positive correlation between antipsychotic medications and GABA levels at right hemisphere. Previous ${ }^{1} \mathrm{H}-\mathrm{MRS}$ studies have reported inconsistent results regarding GABA levels in schizophrenia. In first episode psychosis patients, GABA levels were lower within left basal ganglia (Goto et al., 2010) and bilateral calcarine sulci (Kelemen et al., 2013) and approximately the same within frontal and parieto-occipital lobes (Chowdury et al., 2014) in comparison to healthy controls. Moreover, a study comparing young schizophrenia patients and healthy controls reported that GABA levels were lower within anterior cingulate region for the schizophrenia patients and same within centrum semiovale (Rowland et al., 2013). Whereas in chronic schizophrenia patients, GABA levels were higher within anterior cingulate and parieto-occipital cortices [9], and normal within anterior cingulate cortex and left basal ganglia regions (tayoshi et al., 2010). 
Variations of the GABA levels might be due to differences in brain regions, psychotropic medications, and clinical states in the previous ${ }^{1} \mathrm{H}-\mathrm{MRS}$ studies (Wijtenburg et al., 2015; Brady et al., 2013; Maddock and Buonocore, 2012).

The balance of the excitatory and inhibitory impulses may determine the GABAergic cell activity (Bernstein et al., 2015; Brix et al., 2015). Correlation between GABA and glutamate layers in prefrontal cortices (Brix et al., 2015) might be an indicator of the relationship between excitatory and inhibitory neurotransmission. Since the excitatory neurotransmission is degraded in neurodevelopmental disorders, activity of the GABAergic cells and GABA levels might be altered in order to protect the balance between excitatory and inhibitory neurotransmission (Brix et al., 2015; Ramamoorthi and Lin, 2011; Ren et al., 2012).

In addition, GABA receptors are highly susceptible to rapid neuroplastic changes and various mechanisms such as phosphorylation of synaptic proteins (Stelzer et al., 1986). These findings are suggestive of dynamic modulation of GABAergic neurotransmission according to the dynamics of the synapse or the network. Taken together, these findings may also explain the variability of GABA levels reported in previous studies using ${ }^{1} \mathrm{H}-\mathrm{MRS}$, as GABA neurotransmission is modulated by several clinical factors prone to rapid changes. On the other hand, GABA receptors are often not saturated (Cohen et al., 2000) and therefore the determinant of GABAergic signaling is synthesis of GABA from glutamate (Lazarus et al., 2015). Therefore, activity level of the enzyme glutamic-acid decarboxylase (GAD) 65 and 67 isoenzymes, which catalyze the rate limiting step of GABA synthesis, determine the level of GABAergic activity. Although postmortem studies have reported decreased expression of GAD67 (Lewis et al., 2005), several long term modulations may also alter GABAergic signaling as well as short term changes (Sigel and Steinman, 2012) and deficiency of GAD67 might be compensated upon long term modulations (Lazarus et al., 2015).

Abnormalities in the left hemispheric auditory cortices are associated with linguistic functions and specific symptoms of psychotic spectrum disorders (Mccarley et al., 2002; Jardri et al., 2011; Atagun et al., 2015; Atagun, 2016) and developmental disorders (Rojas et al., 2016). However, GABA levels were higher in right hemisphere in schizophrenia and were positively correlated with antipsychotic doses. This finding suggests that antipsychotics could have enhanced GABA levels only at right hemisphere and could not enhanced left hemisphere GABA levels due to a stronger neuropathology in left hemisphere. In addition, valproate serum levels were correlated with GABA levels at right hemisphere in bipolar disorder. This is in line 
with a previous study [8], which has reported that mood stabilizer anticonvulsants adjunctive to antipsychotics have increased GABA levels at parieto-occipital lobe in schizophrenia. On the other hand, studies investigating the relationship between antipsychotics and GABA levels have been indicated both decrease (Tayoshi et al., 2010; Mao, 2012) and no effect (Öngür et al., 2011; Goto et al., 2010) in schizophrenia, as a result of the medication. Further controlled studies with specific designs to investigate the effects of medications on GABA levels are needed to obtain more consistent and reliable results using ${ }^{1} \mathrm{H}-\mathrm{MRS}$. A current concept suggests that antipsychotics may restore the disturbances (disrupted myelination, reverse the loss of dendritic spines, enhance synaptic connections) of the excitatory neurotransmission that projects to GABAergic cells and stimulate oligodendrocyte maturation (Mao, 2012) and increase the efficiency of GABAergic cells (Bernstein et al., 2015). To this end, antipsychotics may ameliorate the pathology of the GABAergic cells in schizophrenia and bipolar disorder. However, it is not possible to predict the ultimate effects of antipsychotics on GABAergic functions currently, as there are several other determinants for GABAergic functions (such as excitatory/inhibitory balance, receptor phosphorylation, ion channel physiology), but yet GABA level is currently the only measurable in vivo response of the cell.

Although cortical GABA content as quantified by ${ }^{1} \mathrm{H}-\mathrm{MRS}$ has been found to predict the functional status of GABA-mediated processes in previous neurophysiology and pharmacological studies (Maddock and Buonocore, 2012), normal GABA levels do not imply regulated GABAergic function. Determinants of GABA levels and mechanisms of compensatory changes in GABAergic activity are future directions for further clarification of GABAergic abnormalities in schizophrenia and bipolar disorder. While ${ }^{1} \mathrm{H}-\mathrm{MRS}$ utilizing edited pulse sequences such as MEGAPRESS is considered a reliable and reproducible method for measuring brain GABA (Bogner et al. 2010), ${ }^{1} \mathrm{H}-\mathrm{MRS}$ cannot discriminate between intra and extracellular GABA levels. Therefore, these results should be viewed cautiously.

To conclude, higher GABA levels observed in the right auditory cortex of schizophrenia patients could be a compensatory mechanism to obtain the balance between excitatory and inhibitory impulses in the cerebral cortex. Due to pharmacological and physiopathological influences on this balance, in this investigation, we may be capturing a certain phase of GABA metabolism that could be modulated in a dynamic process. Dynamic modulation of the GABAergic activity might be the underlying reason of the variable results of the ${ }^{1} \mathrm{H}$-MRS studies measuring GABA levels. 
Acknowledgement: We would like to thank Prof. Dr. Ergin Atalar from Bilkent University (Turkey) and Ali Avcı from Siemens, Turkey. We also appreciate technical help regarding voxel segmentation provided by Dinesh Deelchand, Dr. Uzay Emir and Dr. Gülin Öz from the Center for Magnetic Resonance Research, Minneapolis, MN, USA.

Funding: This study was funded by Scientific Research Projects Committee of the Ankara Yıldırım Beyazıt University (Project No: 803), and NIMH grant to CMM (MH073998) and K24 MH104449 from the NIH to DÖ. Dr. Phillips acknowledged the support of the Pittsburgh Foundation.

\section{References}

Andreasen, N.C., 1984. The scale for the assessment of positive symptoms (SAPS), University of Iowa Iowa City IA.

Andreasen, N.C., 1983. The scale for assessment of negative symptoms (SANS), University of Iowa Iowa City IA.

Atagün, M.I., Ş1koğlu, E.M., Can, S.S., Karakaş-Uğurlu, G., Ulusoy-Kaymak, S., Çayköylü, A. Phillips, M., Moore, C., Ongur, D., 2015., Investigation of Heschl's gyrus and planum temporale in patients with schizophrenia and bipolar disorder: A proton magnetic resonance spectroscopy study. Schizophr Res. 161; 202-209.

Atagün, M.I., 2016. Brain oscillations in bipolar disorder and lithium-induced changes. Neuropsychiatr Dis Treat. 12; 589-601.

Bartos, M., Vida, I., Jonas, P., 2007. Synaptic mechanisms of synchronized gamma oscillations in inhibitory interneuron networks. Nat Rev Neurosci. 8; 45-56.

Benes, F.M., Berretta, S., 2001. GABAergic interneurons: implications for understanding schizophrenia and bipolar disorder. Neuropsychopharmacology. 25;1-27.
Bernstein, H.G., Steiner, J., Guest, P.C., Dobrowolny, H., Bogerts, B., 2015. Glial cells as key players in schizophrenia pathology: recent insights and concepts of therapy. Schizophr Res. 161; 4-18.

Bhagwagar, Z., Wylezinska, M., Jezzard, P., Evans, J., Ashworth, F., Sule, A., Matthews, P.M., Cowen, P.J., 2007. Reduction in occipital cortex $\gamma$-aminobutyric acid concentrations in medication-free recovered unipolar depressed and bipolar subjects. Biol Psychiatry. 61; 806-812.

Bogner, W., Gruber, S., Doelken, M., Stadlbauer, A., Ganslandt, O., Boettcher, U., Trattnig, S., Doerfler, A., Stefan, H., Hammen, T., 2010. In vivo quantification of intracerebral GABA by single-voxel $1 \mathrm{H}$ MRS - how reproducible are the results? Eur J Radiol. 73;526-531.

Brady, R.O., Mccarthy, J.M., Prescot, A.P., Jensen, J.E., Cooper, A.J., Cohen, B.M., Renshaw, P.F., Ongur, D., 2013. Brain gamma-aminobutyric acid (GABA) abnormalities in bipolar disorder. Bipolar Disord. 15 ; 434-439.

Brix, M.K., Ersland, L., Hugdahl, K., Grüner, R., Posserud, M.-B., Hammar, Å., Craven, 
A.R., Noeske, R., Evans, C.J., Walker, H.B., Midtvedt, T., Beyer, M.K., 2015. Brain MR spectroscopy in autism spectrum disorderthe GABA excitatory/inhibitory imbalance theory revisited. Front Hum Neurosci. 9:365.

Chowdhury, F.A., O'gorman, R.L., Nashef, L., Elwes, R.D., Edden, R.A., Murdoch, J.B., Barker, G.J., Richardson, M.P., 2014. Investigation of glutamine and GABA levels in patients with idiopathic generalized epilepsy using MEGAPRESS. J Magn Reson Imaging. 41;694-699.

Cochran, D.M., Sikoglu, E.M., Hodge, S.M., Edden, R.A., Foley, A., Kennedy, D.N., Moore, C.M., Frazier, J.A., 2015. Relationship among glutamine, $\gamma$ aminobutyric acid, and social cognition in autism spectrum disorders. J Child Adolesc Psychopharmacol. 25;314-322.

Cohen, A.S., Lin, D.D., Coulter, D.A., 2000. Protracted postnatal development of inhibitory synaptic transmission in rat hippocampal area CA1 neurons. J Neurophysiol. 84;2465-2476.

Dydak, U., Jiang, Y.M., Long, L.L., Zhu, H., Chen, J., Li, W.M., Edden, R.A, Hu, S., Fu, X., Long, Z., Mo, X.A., Meier, D., Hareziak, J., Aschner, M., Murdoch, J.B., Zheng, W., 2010. In Vivo measurement of brain GABA concentrations by magnetic resonance spectroscopy in smelters occupationally exposed to manganese. Environ Health Perspect. 119; 219-224.

Edden, R.A., Barker, P.B., 2007. Spatial effects in the detection of $\gamma$-aminobutyric acid: Improved sensitivity at high fields using inner volume saturation. Magn Reson Med. $58 ; 1276-1282$.

Edden, R.A., Puts, N.A., Harris, A.D., Barker, P.B., Evans, C.J., 2013. Gannet: A batchprocessing tool for the quantitative analysis of gamma-aminobutyric acid-edited MR spectroscopy spectra. J Magn Reson Imaging. 40;1445-1452.

First, M.D., Gibbon, M., Spitzer, R.L., Robert, L., Gibbon, M., Williams, J.B.W., 1996. User's guide for the structured interview for DSM-IV axis I disorders research version (SCID-I, version 2.0), Biometrics Research. New York.

Gandal, M.J., Nesbitt, A.M., Mccurdy, R.M., Alter, M.D., 2012. Measuring the maturity of the fast-spiking interneuron transcriptional program in autism, schizophrenia, and bipolar disorder. PLoS ONE. 7; e41215.

Gonzalez-Burgos, G., Fish, K.N., Lewis, D.A., 2011. GABA neuron alterations, cortical circuit dysfunction and cognitive deficits in schizophrenia. Neural Plast. 2011;723184.

Goto, N., Yoshimura, R., Kakeda, S., Moriya, J., Hori, H., Hayashi, K., Ikenouchi-Sugita, A., Nakano-Umene, W., Katsuki, A., Nishimura, J., Korogi, Y., Nakamura, J., 2010. No alterations of brain GABA after 6 months of treatment with atypical antipsychotic drugs in early-stage firstepisode schizophrenia. Prog Neuropsychopharmacol Biol Psychiatry. 34;1480-1483.

Ishikawa, M., Mizukami, K., Iwakiri, M., Asada, T., 2005. Immunohistochemical and immunoblot analysis of gammaaminobutyric acid $B$ receptor in the prefrontal cortex of subjects with schizophrenia and bipolar disorder. Neurosci Lett. 383;272-277.

Jardri, R., Pouchet, A., Pins, D., Thomas, P., 2011. Cortical activations during auditory verbal hallucinations in schizophrenia: a coordinate-based meta-analysis. Am J Psychiatry. 168; 73-81.

Kaufman, R.E., Ostacher, M.J., Marks, E.H., Simon, N.M., Sachs, G.S., Jensen, J.E., 
Renshaw, P.F., Pollack, M.H., 2009. Brain GABA levels in patients with bipolar disorder. Prog Neuropsychopharmacol Biol Psychiatry. 33; 427-434.

Kelemen, O., Kiss, I., Benedek, G., Kéri, S., 2013. Perceptual and cognitive effects of antipsychotics in first-episode schizophrenia: The potential impact of GABA concentration in the visual cortex. Prog Neuropsychopharmacol Biol Psychiatry. 47;13-19.

Lazarus, M.S., Krishnan, K., Huang, Z.J., 2015. GAD67 deficiency in parvalbumin interneurons produces deficits in inhibitory transmission and network disinhibition in Mouse prefrontal cortex. Cereb Cortex. 25; 1290-1296.

Lewis, D.A., Hashimoto, T., Volk D.W., 2005. Cortical inhibitory neurons and schizophrenia. Nat Rev Neurosci. 6; 312324.

Maddock, R.J., Buonocore, M.H., 2012. MR Spectroscopic studies of the brain in psychiatric disorders. Curr Top Behav Neurosci. 11; 199-251.

Mao, X., 2012. Elevated prefrontal cortex $\gamma$ aminobutyric acid and glutamate-glutamine levels in schizophrenia measured in vivo with proton magnetic resonance spectroscopy. Arch Gen Psychiatry. 69; 449.

Mccarley, R.W., Salisbury, D.F., Hirayasu, Y., Yurgelun-Todd, D.A., Tohen, M., Zarate, C., 2002., Association between smaller left posterior superior temporal gyrus volume on magnetic resonance imaging and smaller left temporal p300 amplitude in first-episode schizophrenia. Arch Gen Psychiatry. 59;321-331.

Mescher, M., Merkle, H., Kirsch, J., Garwood, M., Gruetter, R., 1998. Simultaneous in vivo spectral editing and water suppression. NMR Biomed. 11;266-272.
Mizukami, K., Sasaki, M., Ishikawa, M., Iwakiri, M., Hidaka, S., Shiraishi, H., Iritani, S., 2000. Immunohistochemical localization of gamma-aminobutyric acid (B) receptor in the hippocampus of subjects with schizophrenia. Neurosci Lett. 283; 101-104.

Moore, D.R., 2002. Auditory development and the role of experience. Br Med Bull. 63; 171-181.

Overall, J.E., 1962. The Brief Psychiatric Rating Scale. Psychol Rep. 10; 799.

Öngür, D., Prescot, A.P., Mccarthy, J., Cohen, B.M., Renshaw, P.F., 2010. Elevated gamma-aminobutyric acid levels in chronic schizophrenia. Biol Psychiatry. 68; 667670.

Provencher, S.W., 1993. Estimation of metabolite concentrations from localized in vivo proton NMR spectra. Magn Reson Med. 30;672-679.

Provencher, S.W., 2001. Automatic quantitation of localized in vivo1 $\mathrm{H}$ spectra with LCModel. NMR Biomed. 14; 260-264.

Ramamoorthi, K., Lin, Y., 2011. The contribution of gabaergic dysfunction to neurodevelopmental disorders. Trends Mol Med. 17;452-462.

Ren, Y., Wang, H., Xiao, L., 2012. Improving myelin/oligodendrocyte-related dysfunction: a new mechanism of antipsychotics in the treatment of schizophrenia? Int $\mathrm{J}$ Neuropsychopharm. 16;691-700.

Rojas, D.C., Singel, D., Steinmetz, S., Hepburn, S., Brown, M.S., 2014. Decreased left perisylvian GABA concentration in children with autism and unaffected siblings. Neuroimage. 86; 28-34.

Rowland, L.M., Edden, R.A., Kontson, K., Zhu, H., Barker, P.B., Hong, L.E., 2013. 
GABA predicts inhibition of frequencyspecific oscillations in schizophrenia. $\mathbf{J}$ Neuropsychiatry Clin Neurosci. 25;83-87.

Sigel, E., Steinmann, M.E., 2012. Structure, function, and modulation of $\operatorname{GABA}(\mathrm{A})$ receptors. J Biol Chem. 287;40224-40231.

Sikoglu, E.M., Navarro, A.A.L., Starr, D., Dvir, Y., Nwosu, B.U., Czerniak, S.M., Rogan, R.C., Castro, M.C., Edden, R.A., Frazier, J.A., Moore, C.M., 2015. Vitamin D 3 supplemental treatment for mania in youth with bipolar spectrum disorders. J Child Adolesc Psychopharmacol. 25;415-424.

Stelzer, A., Kay, A., Wong, R., 1988. GABAAreceptor function in hippocampal cells is maintained by phosphorylation factors. Science. 241;339-341.

Tayoshi, S., Nakataki, M., Sumitani, S., Taniguchi, K., Shibuya-Tayoshi, S., Numata, S., Iga, J., Ueno, S., Harada, M., Ohmori, T., 2010. GABA concentration in schizophrenia patients and the effects of antipsychotic medication: a proton magnetic resonance spectroscopy study. Schizophr Res. 117;83-91.

Torrey, E.F., B.M. Barci, M.J. Webster, J.J. Bartko, J.H. Meador-Woodruff, M.B. Knable., 2005. Neurochemical markers for schizophrenia, bipolar disorder, and major depression in postmortem brains. Biol Psychiatry. 57; 252-260.
Wang, A.Y., Lohmann, K.M., Yang, C.K., Zimmerman, E.I., Pantazopoulos, H., Herring, N., Berretta, S., Heckers, S., Konradi, C., 2011 Bipolar disorder type 1 and schizophrenia are accompanied by decreased density of parvalbumin- and somatostatin-positive interneurons in the parahippocampal region. Acta Neuropathol. $122 ; 615-626$.

Waddell, K.W., Avison, M.J., Joers, J.M., Gore, J.C., 2007. A practical guide to robust detection of GABA in human brain by $\mathrm{J}$ difference spectroscopy at $3 \mathrm{~T}$ using a standard volume coil. Magn Reson Imaging. 25;1032-1038.

Wijtenburg, S.A., Yang, S., Fischer, B.A., Rowland, L.M., 2015. In vivo assessment of neurotransmitters and modulators with magnetic resonance spectroscopy: Application to schizophrenia. Neurosci Biobehav Rev. 51; 276-295.

Young, R.C., Biggs J.T., Ziegler V.E., Meyer D.A., 1978. A rating scale for mania: reliability, validity and sensitivity. $\mathrm{Br} \mathrm{J}$ Psychiatry. 133;429-435.

Hamilton M., 1960. A rating scale for depression. J Neurol Neurosurg Psychiatry. $23 ; 56-62$.

Zhu, H., Edden, R.A.E., Ouwerkerk, R., Barker, P.B., 2011. High resolution spectroscopic imaging of GABA at 3 Tesla. Magn Reson Med. 65;603-609. 
CHAPTER 9

Discussion 
Despite the advances in science, the pathophysiology of bipolar disorder (BD) is still unknown and it is still a debilitating disease. Scientists discussed the diagnostic classification systems of psychiatric disorders, criticized their phenotypic definitions, and highlighted the need for more precise phenotypic traits like biological markers (or biomarkers). Electroencephalography (EEG), magnetic resonance imaging (MRI) and spectroscopy (MRS) are promising methods in identifying the fingerprints of the disease mechanisms and thus biomarkers in psychiatric disorders. However, the brain is a sophisticated system in its structure and function and hence determining a valid and reliable biomarker is a complex process. In respect to current knowledge at the molecular and the circuitry level, it would be over-optimistic to expect a single biomarker in BD. However, potential biomarkers might be utilized for both clinical and research purposes, such as screening subjects with high risk or manifest symptoms. Biomarkers can also be utilized to examine pharmacological processes, such as treatment response or side effect monitoring in neurological and psychiatric diseases. In this thesis, EEG and $1 \mathrm{H}-\mathrm{MRS}$ were the focus of study as potential biomarkers in BD.

\section{Brain Oscillations in Bipolar Disorder}

Neurons synchronize their discharges in the same oscillatory rhythm to facilitate neuronal synchronization, and thereby, to establish networks. Strong links between synchronized oscillations and higher cognitive functions promoted research on neural synchronization in neuropsychiatric disorders (Uhlhaas and Singer, 2006). Several resting-state or task-based EEG studies have been performed in BD. However, the findings have not been consistent, particularly because of confounding factors, such as medication. I aimed to assess event-related oscillations in medication-free patients with BD in order to circumvent such factors (Chapters 2 and 3).

In Chapter 2, event-related theta oscillations were examined in patients with BD. In the Fourier transformed EEG, signal by Power Spectrum analysis showed two different resonances with two different peaks within the theta frequency range (Figure 1 in Chapter 2). Accordingly, the signal was dissected into 4-6 Hz and 6-8 Hz ranges and two theta bands were obtained. In both frequency ranges, patients had significantly lower amplitudes in comparison with healthy controls at frontal, central and temporal electrodes in the responses of event-related stimuli. In evoked oscillations, patients had significantly lower amplitudes at frontal, central, parietal and temporal electrode locations. These findings showed synchronization deficits in theta frequency range in BD. Theta frequency reflects functional integration of medial temporal and frontal 
brain structures into coherent neurocognitive networks (Başar, 1999; Klimesch, 1999; von Stein and Sarnthein, 2000). The neocortical loops are driven to oscillate at theta frequency by the orchestration of the hippocampus. The brain processes novel information in collaboration with memory, which depends on the continuous dialogue between the hippocampus and relevant parts of the cortex. Hippocampal theta oscillations are one of the rare sustained rhythms within this concept. There are correlations between theta oscillations and cognitive performance, in particular learning and memory (Klimesch, 1999). Thus, altered theta responses are likely to represent neurophysiologic correlates of cognitive deficits, in particular memory disturbances (Sakowitz et al., 2000).

Delta waves are slow in frequency $(0.5-4 \mathrm{~Hz})$ but high in amplitude. Spontaneous delta activity is typically observed in deep sleep (stage 3 and 4) and disappears in conscious states in the adult brain. Functional delta oscillations are also implicated in the synchronization of brain activity with autonomic functions, in motivational processes associated with both reward and defensive mechanisms with higher emotional involvement, and in cognitive processes related to attention and the detection of motivationally salient stimuli in the environment (Knyazev, 2012). In addition to homeostatic mechanisms, it is now known that delta oscillations are also related with cognitive functions [attention and memory] (Harmony, 2013) and executive functions (Nacher et al., 2013). Evoked and event-related delta oscillations $(0.5-3.5 \mathrm{~Hz})$ in medicationfree patients with BD are presented in Chapter 3. Event-related delta oscillations were significantly lower in all brain regions in the BD group in comparison with the healthy control group. These findings indicate a synchronization deficit in cognitive tasks in patients with BD. Delta oscillations are diminished in other neuropsychiatric disorders including schizophrenia (Doege et al., 2010; Ergen et al., 2008) and Alzheimer's Disease (Yener et al., 2008; Yener et al., 2012), which are related with cognitive dysfunction. Therefore, the decrease in event-related delta oscillations are proposed to be a common feature of cognitive dysfunction in neuropsychiatric disorders (Guntekin and Basar, 2016). One of the most important differences between delta and theta oscillations are the networks they use. Major differences between their networks are that delta oscillations are associated with the thalamus and the brain stem, whereas theta oscillations originate from the hippocampus. Delta oscillations are associated with attention-related processes, whereas theta oscillations are associated with learning- and memory-related processes.

The relationship between the cognitive task-based (event-related oscillations) EEG studies and neurocognitive performance remains an intriguing question. If the task is able to represent a 
particular cognitive function (e.g. working memory or attention-related processes) than results should be parallel to other tests examining the same cognitive function. However, previous studies investigating the relationship between cognitive performance and brain oscillations had limitations and results were inconsistent (Reinvang, 1999). Previous research also showed that there was no direct association between neurocognitive assessment and event-related potentials in schizophrenia or BD (Kim et al., 2019). It might be due to the methodological limitations in examining cognitive functions or cognitive tasks used in EEG studies, such as ceiling effects. Human brain computation is complex, because there are several interactions between multiple parallel loops. A multitude of confounds make it difficult to reveal the causal association between behavioral outcomes and a specific brain oscillation. Brain oscillations have multiple functions and act as codes or operators for synchronous activity of neuron populations that determine large-scale organization of the brain web. In addition to the frequencies of brain oscillations, amplitudes, duration, time locking, and phases are other characteristics that determine the functions of brain oscillations (Basar et al., 1999). Further studies should be designed to assess other characteristics of brain oscillations and their relationship between psychiatric and cognitive symptoms in BD.

Spontaneous brain functions and evoked or event-related potentials are synchronized activities of brain networks. Most psychotropic drugs influence neurotransmitter systems or ion channels, and consequently, alter neuronal firing patterns. Pharmacological EEG studies have reported that almost all medications have specific effects on evoked and event related potentials, as well as on resting state activity in EEG. Effects of lithium were investigated with resting state EEG studies or evoked potential studies (Atagun, 2016). Task-based studies may also contribute to our knowledge, since tasks are related with specific neural mechanisms. In Chapter 4, effects of lithium in auditory event-related beta oscillations were reported. Power spectrum analysis showed that the lithium group had significant increase around $20 \mathrm{~Hz}$. The lithium group had significantly higher beta oscillations in comparison with the healthy control group and medication free patients with BD. Furthermore, serum lithium levels were correlated with amplitudes of beta oscillations in patients with BD. It was later reported that visual event related beta oscillations are increased in $\mathrm{BD}$ on lithium therapy compared with medication free patients with BD and healthy controls (Tan et al., 2016). Together with our study, findings of Tan and colleagues (Tan et al. 2016) support widespread brain effects of lithium. Even though the networks of the visual system are different from the networks of the auditory system, similar patterns in beta responses show that lithium is involved with a mechanism that is independent 
of the sensory system. Correlation between serum lithium levels and amplitudes of eventrelated beta oscillations might be an important finding for future studies which might be related with pharmacological or biophysical mechanisms such as cortical excitability (Atagun, 2016). Elevated background brain activity leads to increased signal-to-noise ratio (SNR) and thus enhanced baseline EEG activity in schizophrenia and BD. This alteration is more evident in medication free patients and $\gamma$-aminobutyric acid (GABA) or glutamatergic dysfunction is suggested to be one of the explanations (Gandal et al., 2012). It is considered that lithium decreases SNR values by inhibiting presynaptic alpha-2 receptors (Lenox and Hahn, 2000). Moreover, lithium stimulates neuroplasticity, which in turn drives networks to oscillate at beta oscillations instead of gamma oscillations. Another reason for enhanced beta oscillations in the lithium group might be an excessive activation of the neuron groups in cognitive networks. In addition, considering that atypical antipsychotics (Ren et al., 2013) and lithium (Meffre et al., 2015) stimulate the maturation of oligodendrocytes, along with the synthesis and maturation of myelin, it is possible that these drugs provide better GABAergic activity, and thus, potentiate fast frequency oscillations. Further studies may evaluate clinical responses to lithium and effects of lithium on brain oscillations, as there were associations between serum lithium levels and amplitudes of event-related beta oscillations (Atagun et al., 2015a).

Sensory gating (SG) is defined as the reduction of the irrelevant stimuli from the environment by the elimination of unattended stimuli. SG can be examined with the auditory dual-click paradigm in EEG. Reduction of the amplitude of the second stimulus is thought to be related to the gating function and the dysfunction of SG may lead to cognitive disruption and functioning (Freedman et al., 1983). Several studies have shown dysfunction of SG mechanism in BD (Cheng et al., 2016). In Chapter 5, a meta-analysis of P50 SG studies was performed in order to determine the effects of diagnosis, number of episodes, and medications in $\mathrm{BD}$ and schizophrenia. Our results showed that SG ratio (S2 amplitudes/S1 amplitudes) and SG difference (S1 amplitudes-S2 amplitudes) were significantly altered in schizophrenia and BD patients, and their first-degree relatives. There was no significant difference between schizophrenia and first-episode psychosis; however, there was significant difference between acute episodes and remission in BD. Medication improved P50 SG alterations significantly in schizophrenia, whereas trend-level changes were observed in BD. Within BD patients, no significant difference was observed between patients with or without psychotic symptoms. These results showed that medications improved SG alterations in schizophrenia and this is in agreement with an earlier hypothesis (Freedman et al., 1983). Although more evidence is 
needed for confirmation, medication seems to improve $\mathrm{SG}$ in patients with BD. Heterogeneity could not be explained by the effects of modifiers such as medication status, diagnostic groups, stimulus duration, and stimulus intensity, and the results remained heterogeneous. Although the findings might be influenced by the heterogeneity of the sample to a degree, it can be suggested that SG is a pertinent measure of illness state in schizophrenia and BD. Medicated and medication-free patients were compared in this study and mixed medication groups were pooled. In order to explore the specific effects of different psychotropic medications, specific designs are needed. Future studies may also assess the longitudinal course of SG alterations in mental disorders and their relationship to clinical aspects.

\section{Neurochemistry of Auditory Cortices in Bipolar Disorder}

The auditory cortices are located on superior temporal lobes (STL), which have been associated with auditory hallucinations (Dierks et al., 1999; Shinn et al., 2013) and thought disorders (Seese et al., 2011; Shenton et al., 1992) in schizophrenia. Both postmortem studies (McNamara et al., 2014; Nudmamud et al., 2003) and MRI studies (Modinos et al., 2013; Vita et al., 2012) reported volumetric loss in STL in patients with schizophrenia. Longitudinal studies have shown progressive volumetric changes in the STL in schizophrenia (Vita et al., 2012). Furthermore, volumetric changes were related with alterations in amplitudes of the P300 potentials (Salisbury et al., 1998) and mismatch negativity (MMN) reduction (Salisbury et al., 2007). These findings consistently showed that the STL is a highly relevant brain region for schizophrenia as well as auditory hallucinations. The research domain criteria (RDOC) approach encourages using symptom dimensions as phenotypes instead of the current diagnostic categories (Cuthbert, 2014; Keshavan and Ongur, 2014). Accordingly, auditory hallucinations might be a transdiagnostic symptom domain related with a specific mechanism and anatomical localization. The STL is a relevant brain region for auditory verbal hallucinations even in non-clinical populations without psychiatric diagnosis (Linden et al., 2011).

Converging evidence suggests that STL is also a relevant brain region for $\mathrm{BD}$, similar to schizophrenia. Auditory hallucinations, cognitive symptoms such as verbal learning/memory deficits, and speech abnormalities such as disorder of associations or accelerated speech indicate abnormalities in auditory cortices in BD. Since thoughts are formed by words and their semantic representations, they are associated with linguistic functions. Moreover, STLs host auditory cortices that are important components of language-related networks. Significant cortical thinning in the superior temporal lobes (Hanford et al., 2016), as well as auditory 
processing deficits in functional studies (Chitty et al., 2013) suggest abnormalities in the STL in BD. Postmortem studies of BD patients also revealed metabolic abnormalities within the STL, such as reduced levels of fatty acids, linoleic acid, arachidonic acid (McNamara et al., 2014), and N-acetylaspartate (NAA) (Nudmamud et al., 2003). Because no study has assessed neurochemistry of BD, we studied neurochemistry in superior temporal gyrus (STG) with ${ }^{1} \mathrm{H}$ MRS in schizophrenia and BD type I comparatively (Chapter 6). A point-resolved spectroscopy sequence (PRESS) was applied around the voxel as implemented to the 3.0 Tesla MR system with a thirty-two channel phased-array head coil, and metabolites levels were quantified. The basis set used for this study included alanine, aspartate, creatine $(\mathrm{Cr})$, phosphocreatine (PCr), GABA, glucose, glutamate (Glu), glutamine (Gln), glycerophosphocholine (GPC), phosphocholine (PCh), glutathione (GSH), inositol (Ins), lactate, NAA, N-acetylaspartyl glutamate (NAAG), scylloinositol, and taurine, as well as macromolecules and lipids. Total NAA (tNAA, NAA and NAAG), Glu, Glx (Glu and Gln), total $\mathrm{Cr}(\mathrm{tCr}, \mathrm{Cr}$ and $\mathrm{PCr}$ ), total choline ( $\mathrm{tCh}, \mathrm{GPC}$ and $\mathrm{PCh}$ ), and Ins were quantified. There were significant differences between schizophrenia, $\mathrm{BD}$, and healthy control groups in the left STG but not the right STG. The schizophrenia group had lower Glu levels in comparison with the healthy control group and this difference was at trend level $(\mathrm{p}=0.10)$. The BD group had significantly lower levels of glutamate $(\mathrm{p}<0.001)$, NAA $(\mathrm{p}=0.004), \mathrm{Cr}(\mathrm{p}=0.008)$ and Ino $(\mathrm{p}=0.003)$ in comparison with the healthy control group. Moreover, the BD group had lower $\mathrm{Cr}$ $(p=0.016)$ and Ins $(p=0.001)$ levels in comparison with the schizophrenia group. Furthermore, age at disease onset, duration of disease, and medication status were covariates of the metabolites. Covariates affected the results for NAA levels in the left hemisphere STL. In addition, metabolite levels were correlated with disease severity estimates and symptoms both in schizophrenia and BD. Depression severity correlated with NAA, Cr and Ino in both right and left STL in the BD group, whereas left hemisphere glutamate, NAA and Cr correlated with negative and positive symptoms in the schizophrenia group.

Our findings are in line with previous studies showing that glutamatergic metabolites are increased in the early phases of psychosis onset in schizophrenia but decreased in chronic, long term schizophrenia (Maddock and Buonocore, 2012; Poels et al., 2014). In BD, glutamatergic compounds are increased in acute episodes, but there were no significant changes in remission (Yuksel and Ongur, 2010). In this first study investigating the STL in BD, we reported for the first time that the left STL was a brain region related with a psychosis dimension (auditory hallucinations) of BD. Unfortunately, our study (Atagun et al., 2015b) did not have sufficient 
power to analyze the relationship between the history of psychosis or hallucinations and the metabolite levels in the STL in the BD group. However, we compared neurochemistry in type I and type II BD with healthy controls in Chapter 7. BD type II patients did not have any metabolite differences compared with healthy controls. However, type I patients with BD had significantly lower levels of Glu, Glx, mI, Cr, and NAA in the left hemisphere STL compared with the type II BD and healthy control groups. In addition, patients with a history of psychosis had lower metabolite levels compared with the patients who had no history of psychotic episodes in this study (Atagun et al., 2018). These findings show that the STL is a critical region for psychosis dimension in BD.

Although Glu levels were significantly lower in BD and schizophrenia than in healthy controls, no significant difference was observed in Glx levels. This finding may indicate that Gln levels are not decreased in BD and schizophrenia. Glu to Gln cycle serves for glutamatergic neurotransmission. Glial cells reuptake Glu from the synaptic cleft and convert Glu to Gln. Gln is transferred to neuron and converted to Glu in the neuron cytoplasm and transferred into the vesicle. This turn-over is maintained by glial cells and neurons together and Glu to Gln ratio is approximately 40/60. Glial dysfunction may disrupt the Glu-Gln balance and in turn glutamatergic neurotransmission in schizophrenia and BD (Maddock and Buonocore, 2012). Vital cellular functions might be preserved to some extent; however, glutamatergic abnormalities may cause dysfunction in information processing capabilities of neurons. For example, MMN abnormalities are related with glutamatergic abnormalities as mentioned above (Chitty et al., 2015; Kaur et al., 2019). Decrease of P3a amplitudes were related with Glu/Gln ratio (Hall et al., 2015b). Finally, auditory steady state response abnormalities in schizophrenia and $\mathrm{BD}$ are believed to be a consequence of glutamatergic abnormalities in the auditory cortices (Javitt, 2009).

$\mathrm{Cr}, \mathrm{mI}$ and NAA were also decreased in BD. NAA is a marker of mitochondrial function of oxidative phosphorylation and thus neuronal viability. Decreased levels of NAA may indicate neuronal dysfunction (Moffett et al., 2007). As such, $\mathrm{Cr}$ and phosphocreatine are phosphorous compounds that transfer energy and decreased levels may indicate energy scarcity in the brain (Maddock and Buonocore, 2012). Decreased Cr levels in BD and schizophrenia might be related to the dysfunction of energy metabolism in the brain (Ongur et al., 2009).

Finally, in Chapter 8, GABA levels within the STLs and inferior parietal lobe were assessed in patients with schizophrenia and BD. Since voxel size of the MEGAPRESS sequence is much larger than the PRESS sequence, voxels contained a larger volume including STLs, and inferior 
parietal lobes which contain auditory belt and parabelt regions (Rauschecker and Scott, 2009). There was no significant difference in GABA levels between schizophrenia and BD in the left superior temporal and inferior parietal region; however, GABA levels were increased in the right hemisphere. In addition, GABA levels were correlated with chlorpromazine equivalent doses of antipsychotics in all antipsychotic users (in all patients including schizophrenia and BD) and serum levels of valproate correlated with GABA levels in BD. GABAergic neurotransmission is dynamically modulated by several mechanisms to keep excitatory and inhibitory impulses in balance (Stelzer et al., 1988). Excitatory neurotransmission that stimulate GABAergic interneurons is improved by antipsychotics by stimulating myelin synthesis and maturation of the innervating neurons' axon (Ren et al., 2013). Antipsychotics are suggested to ameliorate dysfunction of the GABAergic interneurons and restore GABAergic functions and increase efficiency of GABAergic cells. Patients of our study were all medicated and medication may have enhanced GABA levels in our patient groups. Since mood stabilizers and antipsychotics influence GABAergic function (Brady et al., 2013), further studies with ${ }^{1} \mathrm{H}-\mathrm{MRS}$ MEGAPRESS sequence are needed to evaluate GABA levels in patients with BD and schizophrenia and its relationship with medication.

\section{Abnormalities of Auditory Cortices in Bipolar Disorder}

The superior temporal lobes host primary and secondary auditory cortices. Primary auditory cortices consist of receptive cells that have been aligned according to a tonotopic map. Accordingly, sounds are processed in different fields according to their physical characteristics in the primary auditory cortex. Secondary auditory cortices (also called Wernicke's Area) are even more complex association centers of the auditory system. The auditory system provides basis for complex linguistic functions in humans. Such state-of-the-art linguistic functions are unique to human beings in nature and the involved brain regions are highly sophisticated than in other species. Linguistic functions provide a basis for thought in humans and since the auditory cortices are components of linguistic functions, they are associated with thought disorders (such as delusions) as well as auditory hallucinations (Humpston et al., 2019). However, auditory cortices have been frequently examined in schizophrenia but rarely in BD.

The development of auditory cortices is a long process - the maturation of the auditory network prolongs until late adolescence (Moore, 2002). For example, myelin sheaths of the thalamocortical projections to auditory cortices begin to mature in the first four years of life. Production of mature neurofilaments for the axonal skeleton prolong until the age of ten. Consistently, prolonged developmental processes neurophysiological maturation follows the 
abovementioned developments. Particularly, event-related potentials P1 and N1 are thought to be related with the primary auditory cortices (Ponton et al., 2002) and since P1, N1 and P2 responses appear around the ages of fifteen, it can be stated that the development of the auditory networks continue until late adolescence. Because of this slow and delicate developmental trajectory, auditory networks are vulnerable to neuropathological processes more than rapidly maturing networks, such as motor networks. As such, disruption of the brain development during the onset of BD (MacCabe et al., 2013; Pavuluri et al., 2009) may also transpire in the auditory cortices, since auditory cortices are particularly vulnerable to developmental lag. Taken together, anatomical location and diversity of functional assessment tools make STL an interesting brain region for further research in major mental disorders, including BD. Several lines of evidence from postmortem studies (McNamara et al., 2014; Nudmamud et al., 2003), neuroimaging (Atagun et al., 2019; Atagun et al., 2015b; Chitty et al., 2015; Hanford et al., 2016; Hirayasu et al., 1998), and EEG studies (Cheng et al., 2016; Chitty et al., 2013; Hamm et al., 2013; Kaur et al., 2019; Parker et al., 2019) point out the STL in BD within this context.

\section{Conclusions, Limitations and Future Directions}

Brain oscillations under task conditions may show relationships between synchronization capabilities of the cognitive networks. Patients with BD exhibited reduced amplitudes in delta and theta oscillations. Functioning of the early auditory system can be assessed using ERPs, which trace sequential activation of the auditory system from brainstem through auditory thalamus (medial geniculate nucleus) and primary auditory cortex (A1) to auditory association cortices. In addition to auditory networks, cognitive task-related networks are also examined for their event-related synchronization capabilities in task-based EEG studies. The changes causing synchronization capabilities may also be related with chemical changes. Further studies may investigate this relationship. Three studies in BD have already employed both ${ }^{1} \mathrm{H}-\mathrm{MRS}$ and EEG and reported associations between MMN or P300 alterations and chemical changes. However, it is still not clear whether there is a relationship between changes in GABA levels and deficits in gamma oscillations; neither are the differential effects of medications on neurophysiology and chemistry. $\mathrm{BD}$ is characterized by numerous neurotransmitter abnormalities and cognitive dysfunction. Therefore, task-based EEG studies may provide information about abnormalities of these alterations. Reduced amplitudes are a common feature of EEG studies in both schizophrenia and BD (Atagun, 2016). These findings indicate neural synchronization deficits in auditory and cognitive cortices; and medications seem to alleviate the deficits. 
Auditory cortices are critical areas in the cortex, since they are associated with the pathophysiology of auditory hallucinations. Both patients with schizophrenia and BD had alterations in their auditory cortices. Metabolites in the ${ }^{1} \mathrm{H}-\mathrm{MRS}$ are decreased in STLs in schizophrenia and BD. BD type I and II differed in terms of metabolites. Patients with type I BD had more alterations than patients with type II BD.

There are several limitations to these studies. First of all, various potential confounders including medications, metabolic disturbances, obesity, nicotine, alcohol or substance use, and medical and psychiatric comorbidities may have affected the results. For example, daily doses of medications and serum levels were associated with the measurements either in EEG or ${ }^{1} \mathrm{H}$ MRS. Metabolite levels were measured in a limited volume in STLs in ${ }^{1} \mathrm{H}-\mathrm{MRS}$. Longitudinal studies might prove more informative than cross-sectional ones. Examining patients in different states may also shed light into state-related changes in BD. More multimodal studies that combine ${ }^{1} \mathrm{H}$-MRS and EEG should be performed in order to determine the role of specific metabolites in task-based EEG studies. The meta-analysis study had heterogeneity and publication-bias limitations. Both clinical and methodological heterogeneity limited the results. The EEG is an optimal tool to estimate functional processes because of its high temporal resolution. Event-related task-based studies showed auditory processing abnormalities in BD. Neurochemical alterations in the auditory cortices might be related with the auditory processing deficits in BD. Pharmacological modulations could be monitored by EEG and neuroimaging modalities. For example, lithium induced EEG changes are promising biomarkers for BD. Classical analysis techniques might be improved by artificial intelligence methods such as machine learning approaches. Clinical utility as biomarkers might be possible with such improved analysis techniques. Future studies with longitudinal designs and larger sample sizes may identify causal relationships.

\section{References}

Atagun, M.I., 2016. Brain oscillations in bipolar disorder and lithium-induced changes. Neuropsychiatr Dis Treat. 12, 589-601.

Atagun, M.I., Akin, B., Algin, O., Şıkoğlu, E.M., Can, S.S., Ulusoy Kaymak, S., Caykoylu, A., Moore, C.M., Phillips, M., Ongur, D., 2019. Intrinsic oscillations of auditory networks in schizophrenia and bipolar disorder. Dusunen Adam. Journal of
Psychiatry and Neurological Sciences. 32 (4), 4-13.

Atagun, M.I., Guntekin, B., Tan, D., Tulay, E.E., Basar, E., 2015a. Lithium excessively enhances event related beta oscillations in patients with bipolar disorder. J Affect Disord. 170, 59-65. 
Atagun, M.I., Sikoglu, E.M., Can, S.S., Karakas-Ugurlu, G., Ulusoy-Kaymak, S., Caykoylu, A., Algin, O., Phillips, M.L., Moore, C.M., Ongur, D., 2015 b. Investigation of Heschl's gyrus and planum temporale in patients with schizophrenia and bipolar disorder: a proton magnetic resonance spectroscopy study. Schizophr Res. 161 (2-3), 202-209.

Atagun, M.I., Sikoglu, E.M., Can, S.S., Ugurlu, G.K., Kaymak, S.U., Caykoylu, A., Algin, O., Phillips, M.L., Moore, C.M., Ongur, D., 2018. Neurochemical differences between bipolar disorder type I and II in superior temporal cortices: A proton magnetic resonance spectroscopy study. J Affect Disord. 235, 15-19.

Başar, E., 1999. Brain function and oscillations: II. Integrative brain function. Neurophysiology and cognitive processes. Springer, Heidelberg, Germany.

Basar, E., Basar-Eroglu, C., Karakas, S., Schurmann, M., 1999. Are cognitive processes manifested in event-related gamma, alpha, theta and delta oscillations in the EEG? Neurosci Lett. 259 (3), 165-168.

Brady, R.O., Jr., McCarthy, J.M., Prescot, A.P., Jensen, J.E., Cooper, A.J., Cohen, B.M., Renshaw, P.F., Ongur, D., 2013. Brain gamma-aminobutyric acid (GABA) abnormalities in bipolar disorder. Bipolar Disord. 15 (4), 434-439.

Cheng, C.H., Chan, P.S., Liu, C.Y., Hsu, S.C., 2016. Auditory sensory gating in patients with bipolar disorders: A meta-analysis. J Affect Disord. 203, 199-203.

Chitty, K.M., Lagopoulos, J., Hickie, I.B., Hermens, D.F., 2015. Hippocampal glutamatergic/NMDA receptor functioning in bipolar disorder: A study combining mismatch negativity and proton magnetic resonance spectroscopy. Psychiatry Res. 233 (2), 88-94.
Chitty, K.M., Lagopoulos, J., Lee, R.S., Hickie, I.B., Hermens, D.F., 2013. A systematic review and meta-analysis of proton magnetic resonance spectroscopy and mismatch negativity in bipolar disorder. Eur Neuropsychopharmacol. 23 (11), 13481363.

Cuthbert, B.N., 2014. The RDoC framework: facilitating transition from ICD/DSM to dimensional approaches that integrate neuroscience and psychopathology. World Psychiatry. 13 (1), 28-35.

Dierks, T., Linden, D.E., Jandl, M., Formisano, E., Goebel, R., Lanfermann, H., Singer, W., 1999. Activation of Heschl's gyrus during auditory hallucinations. Neuron 22 (3), 615621.

Doege, K., Jansen, M., Mallikarjun, P., Liddle, E.B., Liddle, P.F., 2010. How much does phase resetting contribute to event-related EEG abnormalities in schizophrenia? Neurosci Lett. 481 (1), 1-5.

Ergen, M., Marbach, S., Brand, A., BasarEroglu, C., Demiralp, T., 2008. P3 and delta band responses in visual oddball paradigm in schizophrenia. Neurosci Lett. 440 (3), 304308.

Freedman, R., Adler, L.E., Waldo, M.C., Pachtman, E., Franks, R.D., 1983. Neurophysiological evidence for a defect in inhibitory pathways in schizophrenia: comparison of medicated and drug-free patients. Biol Psychiatry. 18 (5), 537-551.

Gandal, M.J., Edgar, J.C., Klook, K., Siegel, S.J., 2012. Gamma synchrony: towards a translational biomarker for the treatmentresistant symptoms of schizophrenia. Neuropharmacology 62 (3), 1504-1518.

Guntekin, B., Basar, E., 2016. Review of evoked and event-related delta responses in the human brain. Int J Psychophysiol. 103, 43-52. 
Hall, M.H., Jensen, J.E., Du, F., Smoller, J.W., O'Connor, L., Spencer, K.M., Ongur, D., 2015. Frontal P3 event-related potential is related to brain glutamine/glutamate ratio measured in vivo. Neuroimage 111, 186191.

Hamm, J.P., Ethridge, L.E., Shapiro, J.R., Pearlson, G.D., Tamminga, C.A., Sweeney, J.A., Keshavan, M.S., Thaker, G.K., Clementz, B.A., 2013. Family history of psychosis moderates early auditory cortical response abnormalities in non-psychotic bipolar disorder. Bipolar Disord. 15 (7), 774-786.

Hanford, L.C., Nazarov, A., Hall, G.B., Sassi, R.B., 2016. Cortical thickness in bipolar disorder: a systematic review. Bipolar Disord. 18 (1), 4-18.

Harmony, T., 2013. The functional significance of delta oscillations in cognitive processing. Front Integr Neurosci. 7, 83.

Hirayasu, Y., Shenton, M.E., Salisbury, D.F., Dickey, C.C., Fischer, I.A., Mazzoni, P., Kisler, T., Arakaki, H., Kwon, J.S., Anderson, J.E., Yurgelun-Todd, D., Tohen, M., McCarley, R.W., 1998. Lower left temporal lobe MRI volumes in patients with first-episode schizophrenia compared with psychotic patients with first-episode affective disorder and normal subjects. Am J Psychiatry. 155 (10), 1384-1391.

Humpston, C.S., Adams, R.A., Benrimoh, D., Broome, M.R., Corlett, P.R., Gerrans, P., Horga, G., Parr, T., Pienkos, E., Powers, A.R., 3rd, Raballo, A., Rosen, C., Linden, D.E.J., 2019. From Computation to the FirstPerson: Auditory-Verbal Hallucinations and Delusions of Thought Interference in Schizophrenia-Spectrum Psychoses. Schizophr Bull. 45 (45 Suppl 1), S56-S66.

Javitt, D.C., 2009. When doors of perception close: bottom-up models of disrupted cognition in schizophrenia. Annu Rev Clin Psychol. 5, 249-275.

Kaur, M., Chitty, K.M., Lagopoulos, J., Hickie, I.B., Duffy, S.L., Hermens, D.F., 2019. Elucidating the glutamatergic processes underlying mismatch negativity deficits in early stage bipolar disorder and schizophrenia: A combined (1)H-MRS and EEG study. J Psychiatr Res. 113, 83-89.

Keshavan, M.S., Ongur, D., 2014. The journey from RDC/DSM diagnoses toward RDoC dimensions. World Psychiatry 13 (1), 44-46.

Kim, Y., Kwon, A., Min, D., Kim, S., Jin, M.J., Lee, S.H., 2019. Neurophysiological and Psychological Predictors of Social Functioning in Patients with Schizophrenia and Bipolar Disorder. Psychiatry Investig. 16 (10), 718-727.

Klimesch, W., 1999. EEG alpha and theta oscillations reflect cognitive and memory performance: a review and analysis. Brain Res Brain Res Rev 29 (2-3), 169-195.

Knyazev, G.G., 2012. EEG delta oscillations as a correlate of basic homeostatic and motivational processes. Neurosci Biobehav Rev. 36 (1), 677-695.

Lenox, R.H., Hahn, C.G., 2000. Overview of the mechanism of action of lithium in the brain: fifty-year update. J Clin Psychiatry. 61 Suppl 9, 5-15.

Linden, D.E., Thornton, K., Kuswanto, C.N., Johnston, S.J., van de Ven, V., Jackson, M.C., 2011. The brain's voices: comparing nonclinical auditory hallucinations and imagery. Cereb Cortex. 21 (2), 330-337.

MacCabe, J.H., Wicks, S., Lofving, S., David, A.S., Berndtsson, A., Gustafsson, J.E., Allebeck, P., Dalman, C., 2013. Decline in cognitive performance between ages 13 and 18 years and the risk for psychosis in adulthood: a Swedish longitudinal cohort 
study in males. JAMA Psychiatry. 70 (3), 261-270.

Maddock, R.J., Buonocore, M.H., 2012. MR spectroscopic studies of the brain in psychiatric disorders. Curr Top Behav Neurosci. 11, 199-251.

McNamara, R.K., Rider, T., Jandacek, R., Tso, P., 2014. Abnormal fatty acid pattern in the superior temporal gyrus distinguishes bipolar disorder from major depression and schizophrenia and resembles multiple sclerosis. Psychiatry Res. 215 (3), 560-567.

Meffre, D., Massaad, C., Grenier, J., 2015. Lithium chloride stimulates PLP and MBP expression in oligodendrocytes via Wnt/beta-catenin and Akt/CREB pathways. Neuroscience 284, 962-971.

Modinos, G., Costafreda, S.G., van Tol, M.J., McGuire, P.K., Aleman, A., Allen, P., 2013. Neuroanatomy of auditory verbal hallucinations in schizophrenia: a quantitative meta-analysis of voxel-based morphometry studies. Cortex. 49 (4), 10461055.

Moffett, J.R., Ross, B., Arun, P., Madhavarao, C.N., Namboodiri, A.M., 2007. NAcetylaspartate in the CNS: from neurodiagnostics to neurobiology. Prog Neurobiol. 81 (2), 89-131.

Moore, D.R., 2002. Auditory development and the role of experience. Br Med Bull. 63, 171181.

Nacher, V., Ledberg, A., Deco, G., Romo, R., 2013. Coherent delta-band oscillations between cortical areas correlate with decision making. Proc Natl Acad Sci U S A. 110 (37), 15085-15090.

Nudmamud, S., Reynolds, L.M., Reynolds, G.P., 2003. N-acetylaspartate and NAcetylaspartylglutamate deficits in superior temporal cortex in schizophrenia and bipolar disorder: a postmortem study. Biol Psychiatry. 53 (12), 1138-1141.

Ongur, D., Prescot, A.P., Jensen, J.E., Cohen, B.M., Renshaw, P.F., 2009. Creatine abnormalities in schizophrenia and bipolar disorder. Psychiatry Res. 172 (1), 44-48.

Parker, D.A., Hamm, J.P., McDowell, J.E., Keedy, S.K., Gershon, E.S., Ivleva, E.I., Pearlson, G.D., Keshavan, M.S., Tamminga, C.A., Sweeney, J.A., Clementz, B.A., 2019. Auditory steady-state EEG response across the schizo-bipolar spectrum. Schizophr Res. 209, 218-226.

Pavuluri, M.N., West, A., Hill, S.K., Jindal, K., Sweeney, J.A., 2009. Neurocognitive function in pediatric bipolar disorder: 3-year follow-up shows cognitive development lagging behind healthy youths. J Am Acad Child Adolesc Psychiatry. 48 (3), 299-307.

Poels, E.M., Kegeles, L.S., Kantrowitz, J.T., Javitt, D.C., Lieberman, J.A., Abi-Dargham, A., Girgis, R.R., 2014. Glutamatergic abnormalities in schizophrenia: a review of proton MRS findings. Schizophr Res. 152 (2-3), 325-332.

Ponton, C., Eggermont, J.J., Khosla, D., Kwong, B., Don, M., 2002. Maturation of human central auditory system activity: separating auditory evoked potentials by dipole source modeling. Clin Neurophysiol. 113 (3), 407-420.

Rauschecker, J.P., Scott, S.K., 2009. Maps and streams in the auditory cortex: nonhuman primates illuminate human speech processing. Nat Neurosci. 12 (6), 718-724.

Reinvang, I., 1999. Cognitive event-related potentials in neuropsychological assessment. Neuropsychol Rev. 9 (4), 231248.

Ren, Y., Wang, H., Xiao, L., 2013. Improving myelin/oligodendrocyte-related 
dysfunction: a new mechanism of antipsychotics in the treatment of schizophrenia? Int J Neuropsychopharmacol. 16 (3), 691-700.

Sakowitz, O.W., Schurmann, M., Basar, E., 2000. Oscillatory frontal theta responses are increased upon bisensory stimulation. Clin Neurophysiol. 111 (5), 884-893.

Salisbury, D.F., Kuroki, N., Kasai, K., Shenton, M.E., McCarley, R.W., 2007. Progressive and interrelated functional and structural evidence of post-onset brain reduction in schizophrenia. Arch Gen Psychiatry. 64 (5), 521-529.

Salisbury, D.F., Shenton, M.E., Sherwood, A.R., Fischer, I.A., Yurgelun-Todd, D.A., Tohen, M., McCarley, R.W., 1998. Firstepisode schizophrenic psychosis differs from first-episode affective psychosis and controls in P300 amplitude over left temporal lobe. Arch Gen Psychiatry. 55 (2), 173-180.

Seese, R.R., O'Neill, J., Hudkins, M., Siddarth, P., Levitt, J., Tseng, B., Wu, K.N., Caplan, R., 2011. Proton magnetic resonance spectroscopy and thought disorder in childhood schizophrenia. Schizophr Res. 133 (1-3), 82-90.

Shenton, M.E., Kikinis, R., Jolesz, F.A., Pollak, S.D., LeMay, M., Wible, C.G., Hokama, H., Martin, J., Metcalf, D., Coleman, M., et al., 1992. Abnormalities of the left temporal lobe and thought disorder in schizophrenia. A quantitative magnetic resonance imaging study. N Engl J Med. 327 (9), 604-612.

Shinn, A.K., Baker, J.T., Cohen, B.M., Ongur, D., 2013. Functional connectivity of left Heschl's gyrus in vulnerability to auditory hallucinations in schizophrenia. Schizophr Res. 143 (2-3), 260-268.
Stelzer, A., Kay, A.R., Wong, R.K., 1988. GABAA-receptor function in hippocampal cells is maintained by phosphorylation factors. Science 241 (4863), 339-341.

Tan, D., Ozerdem, A., Guntekin, B., Atagun, M.I., Tulay, E., Karadag, F., Basar, E., 2016. Increased Beta Frequency $(15-30 \mathrm{~Hz})$ Oscillatory Responses in Euthymic Bipolar Patients Under Lithium Monotherapy. Clin EEG Neurosci. 47 (2), 87-95.

Uhlhaas, P.J., Singer, W., 2006. Neural synchrony in brain disorders: relevance for cognitive dysfunctions and pathophysiology. Neuron 52 (1), 155-168.

Vita, A., De Peri, L., Deste, G., Sacchetti, E., 2012. Progressive loss of cortical gray matter in schizophrenia: a meta-analysis and meta-regression of longitudinal MRI studies. Transl Psychiatry. 2, e190.

von Stein, A., Sarnthein, J., 2000. Different frequencies for different scales of cortical integration: from local gamma to long range alpha/theta synchronization. Int $\mathbf{J}$ Psychophysiol. 38 (3), 301-313.

Yener, G., Guntekin, B., Basar, E., 2008. Eventrelated delta oscillatory responses of Alzheimer patients. Eur J Neurol. 15 (6), 540-547.

Yener, G.G., Guntekin, B., Orken, D.N., Tulay, E., Forta, H., Basar, E., 2012. Auditory delta event-related oscillatory responses are decreased in Alzheimer's disease. Behav Neurol. 25 (1), 3-11.

Yuksel, C., Ongur, D., 2010. Magnetic resonance spectroscopy studies of glutamate-related abnormalities in mood disorders. Biol Psychiatry. 68 (9), 785-794. 
CHAPTER 10

Summary 


\section{Summary}

In chapter 1, electroencephalography (EEG) and magnetic resonance imaging (MRI) are introduced as promising methods for identifying the fingerprints of pathophysiological mechanisms and thus biomarkers in psychiatric disorders. Pathophysiological mechanisms of bipolar disorder (BD) were summarized. Potential biomarkers might be candidate monitorization tools for both clinical and research purposes, such as screening high risk subjects or those with progression of the symptoms. Pharmacological processes (e.g. treatment response or side effect monitorization) can also be assessed in neurological and psychiatric diseases. The major advantage of the EEG is its high temporal resolution and researchers concentrated on cognitive dysfunction and corresponding imaging findings as candidate biomarkers in BD (Pavuluri, 2010). Studies were designed to assess the relationship between cognitive symptoms and brain structure and cognitive tasks have been utilized for functional assessments.

Chapter 2 and 3 present the examination of cognitive alterations in BD. In cognitive task-based EEG studies, event-related potentials (ERP) are obtained. ERPs are higher in energy compared with evoked potentials and this indicates that higher number of neuron groups is involved with cognitive function. The oddball paradigm is a very simple working memory task that minimizes the risk of confounds. Two types of stimuli (1500 and $1600 \mathrm{~Hz})$ are presented and the subject is asked to discriminate the stimuli and count the rare one (target stimulus, $1600 \mathrm{~Hz}$ ) mentally. Attention and mental counting processes enhance the voltage of the target ERPs. In neuropsychiatric disorders characterized with neurocognitive impairment, voltage reductions are observed (Chapter 2 and 3). Voltage reductions may result from either non-responding neural units or reduced synchronization capabilities in the neural circuits involved with cognitive demands of the task. Congruent with this, long range fronto-temporal connectivity was reduced in BD (Ozerdem et al., 2011).

In addition to the examination of neural network synchronization capabilities, the EEG can be utilized to assess pharmacological processes such as treatment response. Chapter 4 presents the lithium induced changes in beta oscillations in BD. Lithium is the gold standard treatment drug for BD, whose mechanism of effect is not clearly known. Although lithium is more effective in high doses, its therapeutic range is narrow and serum levels are tightly checked in order to avoid its side effects. Lithium is an element and has biophysical and pharmacological properties. Several EEG studies are published but neither of them were cognitive task based studies. I wanted to evaluate the effects of lithium on cognitive networks. We found that lithium excessively enhances event-related beta oscillations upon auditory oddball paradigm (Atagun 
et al. 2015). In a later study, we also found that lithium also enhances event-related beta oscillations upon visual oddball paradigm (Tan et al. 2016). Our interpretation was that, the effect of lithium on cognitive networks is cortex-wide and rather than a general enhancement of cortical excitability, it activates neurons to participate in cognitive activity specifically upon target response (Atagun, 2016). Interestingly, the enhanced amplitudes of the beta responses were correlated with the serum levels of lithium. More studies are needed to evaluate the effects of lithium on brain oscillations in order to determine their relationship between treatment response and possible side effects.

Chapter 5 presents a meta-analysis of P50 sensory gating studies in schizophrenia and BD. Sensory gating is a measure of the integrity of the brain's inhibitory function. It is measured by using the amplitude of the evoked potential at $50 \mathrm{~ms}$ to the first of two paired clicks, divided by the response to the second. Sensory gating is altered in both schizophrenia and BD and their first degree relatives (Chapter 5). Moreover, sensory gating is even more altered in the episodes of the diseases and medications seem to reverse the alteration. Therefore, P50 sensory gating is a potential biomarker for both schizophrenia and $\mathrm{BD}$, since it is coherent with the progress of the diseases.

Chapters 6 and 7 present proton magnetic resonance imaging $\left({ }^{1} \mathrm{H}-\mathrm{MRS}\right)$ studies in patients with BD. The ${ }^{1} \mathrm{H}-\mathrm{MRS}$ in vivo measures major metabolites such as glu, NAA, $\mathrm{mI}, \mathrm{Cr}$ and Cho in the brain. Glu is an amino-acid neurotransmitter and is abundant in the brain. Its cycle as a neurotransmitter is provided by glial cells and dysfunction of the glia may disrupt this process. NAA is a marker of viability in neurons. Cr is related with the energy metabolism, Cho is related with membrane turn-over, and $\mathrm{mI}$ is a compound involved with the inositol cycle. Several studies were performed and it was found that glutamate levels were increased in BD. Other studies showed that Cr levels were altered in BD (Ongur et al., 2009). Most of the studies were performed in frontal and anterior cingulate cortices (Maddock and Buonocore, 2012). However, although BD is characterized by speech abnormalities during episodes, verbal learning/memory deficits and auditory hallucinations in psychotic patients, auditory cortices were not assessed in BD. We aimed to assess primary and secondary auditory cortices in BD type I and compare it with those of schizophrenia (Chapter 6). Patients with BD had reductions in left STL compared with schizophrenia and healthy controls. Furthermore, we also compared BD type I and II and found that patients with BD type II did not show a reduction in metabolite levels in STL compared with BD type I and healthy controls. Only type I BD patients had reductions in metabolite levels (Chapter 7). The ${ }^{1} \mathrm{H}-\mathrm{MRS}$ is also available to detect GABA with 
MEGAPRESS sequence in a given volume. GABA levels can be in vivo assessed by ${ }^{1} \mathrm{H}-\mathrm{MRS}$ in the STL and we did not observe any abnormality in either type I or type II patients with BD (Chapter 8).

Chapter 9 is the discussion section in which the findings are interpreted. One of the most important points of the studies is the alterations of the auditory cortices. Alterations of the auditory cortices are reflected in all neuroimaging modalities as well as neurophysiological examinations. In addition, there are associations between neurophysiological alterations and neuroimaging findings. For example, MMN alterations and STL volumes were found to be correlated (Salisbury et al., 2007; Salisbury et al., 1998). These results suggest that auditory cortices are critical brain regions for patients with BD. According to the RDOC approach, more studies should evaluate the auditory cortices in BD since they are related with specific symptoms in BD. Brain stimulation therapies may add auditory cortices into their programs for the treatment of related symptoms in patients with BD.

\section{References}

Atagun, M.I., 2016. Brain oscillations in bipolar disorder and lithium-induced changes. Neuropsychiatr Dis Treat 12, 589-601.

Maddock, R.J., Buonocore, M.H., 2012. MR spectroscopic studies of the brain in psychiatric disorders. Curr Top Behav Neurosci 11, 199-251.

Ongur, D., Prescot, A.P., Jensen, J.E., Cohen, B.M., Renshaw, P.F., 2009. Creatine abnormalities in schizophrenia and bipolar disorder. Psychiatry Res 172 (1), 44-48.

Ozerdem, A., Guntekin, B., Atagun, I., Turp, B., Basar, E., 2011. Reduced long distance gamma $(28-48 \mathrm{~Hz})$ coherence in euthymic patients with bipolar disorder. J Affect Disord 132 (3), 325-332.
Pavuluri, M.N., 2010. Effects of early intervention on the course of bipolar disorder: theories and realities. Curr Psychiatry Rep 12 (6), 490-498.

Salisbury, D.F., Kuroki, N., Kasai, K., Shenton, M.E., McCarley, R.W., 2007. Progressive and interrelated functional and structural evidence of post-onset brain reduction in schizophrenia. Arch Gen Psychiatry 64 (5), 521-529.

Salisbury, D.F., Shenton, M.E., Sherwood, A.R., Fischer, I.A., Yurgelun-Todd, D.A., Tohen, M., McCarley, R.W., 1998. Firstepisode schizophrenic psychosis differs from first-episode affective psychosis and controls in P300 amplitude over left temporal lobe. Arch Gen Psychiatry 55 (2), 173-180. 
CHAPTER 11

Valorization 


\section{Valorization}

Major psychiatric disorders affect millions of people and they can be severely debilitating. The etiopathology of major psychiatric disorders has not been clearly identified and thus treatment options are yet to be improved. These limitations are because of the complexity of the brain and the heterogeneous nature of psychiatric disorders. Many paradigms and technologies have been adopted and a great amount of research has contributed to the literature. During the last century, psychiatry has evolved towards a multidisciplinary field that collaborates with several fields of basic and applied sciences. Perhaps one of the most invaluable contributing fields is cognitive neuroscience. Several paradigms developed by cognitive neuroscientists have shed light on different brain functions and significantly contributed to psychiatric research.

One of the earliest modalities is electroencephalography (EEG). After Hans Berger made his first experiments in 1929, EEG has been used in psychiatry and neurology for research and clinical purposes. EEG is a cheap and non-invasive method that offers a comprehensive examination of integrative brain function. Electrical activity of the neurons including membrane potentials, rhythmic patterns of action potentials, and postsynaptic discharges produce oscillatory activity at the level of neuron ensembles. Synchronized activity of the large number of neurons gives rise to brain-wide oscillatory activity, which can be recorded by the EEG. Synchronous firing patterns are controlled by feedback loops and interaction between neuron populations may give rise to oscillations at different frequencies. Each frequency could be related with different brain functions.

In the recent years, the National Institute of Mental Health (NIMH) of the USA promoted a research method in psychiatric disorders, called research domain criteria (RDOC). According to this approach, transdiagnostic behavioral and cognitive domains are suggested as target phenotypes instead of categorical psychiatric diagnoses. The auditory system is related with the formation of thought and auditory hallucinations and thus convenient for this research model. Examination of the auditory system has revealed several consistent findings in schizophrenia and bipolar disorders. Accordingly, my thesis work has focused on cognitive mechanisms of auditory networks and linguistic functions, as well as the chemical integrity of the auditory cortices. The innovative aspects of my thesis work are the promising value of associations between disease mechanisms and the dysfunction of the related brain networks. I have demonstrated that cognitive processing of auditory information is disturbed in schizophrenia 
and bipolar disorder. Alterations of the auditory and language networks are associated with the symptoms of schizophrenia and bipolar disorder. Moreover, I have demonstrated that chemical examination of the auditory cortices with magnetic resonance spectroscopy revealed similar alterations in schizophrenia and type I bipolar disorder but not in type II bipolar disorder. These results suggest that there may be shared biological mechanisms between bipolar disorder and schizophrenia, as well as pathophysiological processes that are different between subtypes of bipolar disorder. In this regard, my findings provide support for uniform and distinct pathophysiological mechanisms and add a unique perspective to the Kraepelinian dichotomy of mood and psychotic disorders. Furthermore, these alterations differ in the subtypes of bipolar disorder and schizophrenia, and therefore, they could serve as potential biomarkers in the future. These findings may pave the way for better etiopathology-driven diagnostic classification systems. Moreover, alterations of the auditory networks may show us directions in tailoring personalized treatments. For example, transcranial magnetic stimulation (TMS) is able to alter brain activity by electrical impulses in a given location. Auditory cortices are close to the brain surface and can easily be modulated by TMS. Clinicians are using TMS to modify brain activity in auditory cortices in patients with treatment resistant auditory hallucinations. 
Acknowledgement 


\section{Acknowledgement}

I am grateful to my dear parents and family. They were always with me and always helped me in my work.

I would like to thank to Prof. Dr. Timuçin Oral, director of the clinic I trained for psychiatry, for valuable contributions to my education.

Prof. Dr. Ayşegül Özerdem and Prof. Dr. Erol Başar illuminated my way to academic life.

Prof. Dr. Cuneyt Evren helped me in my personal development and research career.

One of the most important moments of my life was the day I met with Prof. Dr. Dost Öngür. He has been a great mentor and behaved as a member of my family. I am grateful to Professor Öngür for his efforts to show me a bright way.

Sinan Gülöksüz is a lifetime friend with always helping attitude. His team in Maastricht became my team and I am grateful to Sinan for this.

Dr. Marjan Drukker is a great scientist, who taught me very important knowledge.

Professor van Amelsvoort, my chief thesis advisor, is a respected teacher. Despite her authoritative scientific career, my major feeling is warmness and helpful attitude.

Thank you very much 
Curriculum Vitae 


\section{Curriculum Vitae}

Murat İlhan Atagün was born in 1978, April 22th. He graduated from primary school in 1988 and high school in 1996. He had education in medicine in Selçuk University Medical School and graduated in 2003. He worked as a general practitioner in the emergency department of a private hospital. After working 2 years as a general practitioner. he got trained in psychiatry in İstanbul Bakırköy Research and Training Hospital for Psychiatry between 2005 and 2009. He also had training in neurophysiology and neuropsychology during his psychiatry residency in Kültür University Neurophysiology Lab led by Prof. Dr. Erol Başar. He visited Neurophysiology Research Center of the Bremen University led by Prof. Dr. Canan Eroğlu Başar in September and October of 2009. After completing his residency, he worked in İstanbul Bakırköy Research and Training Hospital for Pscyhiatry for two years. Then he moved to Department of Psychiatry, Namık Kemal University Medical School and worked for 1 year. In June 2013, he moved to Ankara Yildirim Beyazit University Medical School and worked until August 2019. During this period, he conducted research in biochemistry and neuroimaging in schizophrenia and bipolar disorder under the supervision of Prof. Dr. Dost Öngür (McLean Hospital and Harvard University). He also had collaborations with Prof. Dr. Mary Phillips (Pittsburgh University Medical School), Prof. Dr. Constance Moore (Universitty of Massachusetts Medical School) and Prof. Dr. Özcan Erel (Ankara Yildirim Beyazit University Medical School). He had been working in Department of Psychiatry, Afyonkarahisar Health Sciences University Medical School since August 2019. 
List of Publications 


\section{List of Publications}

Yumru M, Savas HA, Kurt E, Kaya C, Selek S, Savas E, Oral T, Atagun I. Atypical antipsychotics related metabolic syndrome in bipolar patients. J Affect Disord. 2007;98:247-252.

Özerdem A, Güntekin B, Atagün Mí, Turp Gölbaşı B, Başar E. Reduced Long Distance Gamma (28-48 Hz) Coherence in Euthymic Patients with Bipolar Disorder. J Affect Disord. 2011;132:325-332.

Atagün Mİ, Balaban ÖD, Yeşilbaş D, Keskinkılıç C, Evren C. Lateralization Effect on Motor and Mental Speed in Bipolar Disorder. Bulletin of Clinical Psychopharmacology 2012;22:297-306.

Başar E, Güntekin B, Atagün İ, Turp Gölbaşı B, Tülay E, Özerdem A. Brain’s alpha activity is highly reduced in euthymic bipolar patients. Cogn Neurodyn. 2012:6:11-20.

Atagün Mí, Balaban ÖD, Yeşilbaş D, Evren C. Lithium and Valproate may affect motor and sensory speed in patients with bipolar disorder. Bulletin of Clinical Psychopharmacology $2013 ; 23: 305-14$.

Atagün Mi, Güntekin B, Özerdem A, Tülay E, Başar E. Decrease of Theta Response in Euthymic Bipolar Patients During an Oddball Paradigm. Cogn Neurodyn. 2013;7:21323.

Canbek Ö, Menges OO, Atagün Mi, Kutlar MT, Kurt E. Report on Three Years' Experience in Electroconvulsive Therapy in Bakirkoy Research and Training Hospital for Psychiatric and Neurological Diseases: 2008 - 2010. J ECT 2013;29:51-7.

Atagün Mí, Atagün Z, Doğan S, Evren C, Yılmaz Yalçınkaya E, Öneş K. Fibromiyaljili Kadın Hastalarda Çocukluk Çağı Travmaları ile Cinsel İşlevler Arasındaki İlişki. Anatolian Journal of Psychiatry 2013;14: 200-9.

Balaban ÖD, Atagün Mí, Yılmaz H, Alpkan LR. Benzydamine abuse as a hallucinogen: a case report. Bulletin of Clinical Psychopharmacology 2013; 23:276-9 
Atagün Mí, Oral ET, Sevinç C. Polycystic renal disease in a patient who used lithium for years. Turkish Journal of Psychiatry 2013;24:213-6.

Canbek O, Yuksel G, Atagün Mí, İpekcioğlu D, Kurt E, Menges OO, Kutlar T. Geriatric ECT at a Turkish Teaching Hospital A 5-Year Experience. J ECT 2014;30:e28-9.

Atagün Mí, Güntekin B, Maşalı B, Tülay EE, Başar E. Decrease of event related delta oscillations in euthymic patients with bipolar disorder. Psychiatry Res: Neuroimag 2014;223: 43-48.

Atagün Mí, Güntekin B, Tan D, Tülay E, Başar E. Lithium excessively enhances event related beta oscillations in patients with bipolar disorder. J Affect Disord 2015;170: 59-65.

Can SS, Atagün Mİ, Kabadayı E. A Rare Misidentification Syndrome: Seeing a Monozygotic Twin in the Mirror. Turkish Journal of Psychiatry 2014;25:291-293 DOI: 10.5080/u7897

Canbek Ö, İpekcioğlu D, Mengeş OO, Atagün Mí, Karamustafalığlu N, Çetinkaya ÖZ, İlnem MC. Comparison of Propofol, Etomidate and Thiopental in Anesthesia for Electroconvulsive Therapy: A Randomized, Double-Blind Clinical Trial. J ECT 2015;31(2):91-7. DOI: 10.1097/YCT.0000000000000190.

Atagün Mí, Şıkoğlu EM, Can SS, Karakaş-Uğurlu G, Ulusoy-Kaymak S, .ayk.ylü A, Algın O, Phillips ML, Moore CM, .ngür D. Investigation of Heschl's gyrus and planum temporale in patients with schizophrenia and bipolar disorder: A proton magnetic resonance spectroscopy study. Schizophr Res 2015;161: 202-209.

Tan D, Özerdem A, Güntekin B, Atagün Mí, Tülay EE, Karadağ F, Başar E. Increased Beta Frequency (15-30 Hz) Oscillatory Responses in Euthymic Bipolar Patients under Lithium Monotherapy. Clin EEG Neurosci. 2016;47(2):87-95.

Atagün Mí. Brain oscillations in bipolar disorder and lithium-induced changes. Neuropsychiatr Dis Treat. 2016;12:589-601.

Atagün Mİ, Korkmaz ŞA, Soykan Ç, Büyük.z D, Can SS, .ayk.ylü A. Serum lithium levels are associated with white blood cell counts in bipolar disorder. Turkish Journal of Medical Sciences. 2016; 46: 1271-1272. 
Umut G, Atagun MI, Evren C, Yilmaz-Cengel H, Hisim O, Keskinkilic C. The changes of the cognitive and psychomotor functions in the chronic cannabis users after a month of remission. Bulletin of Clinical Psychopharmacology. 2016; 26: 109-118.

Bağ S, Canbek Ö, Atagün Mi, Kutlar T. Early effects of modern electroconvulsive therapy on subjective memory in patients with mania or depression. Indian Journal of Psychiatry. 2016; 58: 198-203.

Atagün Mi, Şıkoğlu EM, Soykan Ç, Serdar Süleyman C, Ulusoy-Kaymak S, Çayköylü A, Algın O, Phillips ML, ngür D, Moore CM. Perisylvian GABA levels in schizophrenia and bipolar disorder. Neurosci Lett. 2017;637:70-74.

Ulusoy-Kaymak S, Kabadayı Şahin E, Karakaş Uğurlu G, Ünal Ö, Atagün Mi், Can SS, Çayköylü A. Situs Inversus Totalis and Schizophrenia Comorbidity. Turkish Journal of Psychiatry 2017;28(4):287-90 doi: 10.5080/u20549

Atagün Mİ, Şıkoğlu EM, Can SS, Uğurlu GK, Kaymak SU, Çayköylü A, Algın O, Phillips ML, Moore CM, Öngür D. Neurochemical differences between bipolar disorder type I and II in superior temporal cortices: A proton magnetic resonance spectroscopy study. $\mathbf{J}$ Affect Disord. 2018 Aug 1;235:15-19. doi: 10.1016/j.jad.2018.04.010.

Atagün Mí, Tunç S, Alışık M, Erel Ö. Phenotypic Variants of Paraoxonase Q192R in Bipolar Disorder, Depression and Schizophrenia. Turkish Journal of Psychiatry 2018 Summer;29(2):79-86.

Apaydin Z, Atagun MI. Relationship of functionality with impulsivity and coping strategies in bipolar disorder. Dusunen Adam: The Journal of Psychiatry and Neurological Sciences 2018:31 (2); 21-29.

Balaban OD, Atagun Mi, Ozguven HD. Information processing abnormalities in schizophrenia and bipolar disorder. Dusunen Adam: The Journal of Psychiatry and Neurological Sciences 2018: 31 (2); 135-147.

Tunç S, Atagün Mİ, Neşelioğlu S, Bilgin YY, Başbuğ HS, Erel Ö. Ischemia-modified albumin: a unique marker of global metabolic risk in schizophrenia and mood disorders. Psychiatry and Clinical Psychopharmacology 2019:29 (2); 123-129. 
Atagün Mí, Akın B, Algın O, Sıkoğlu EM, Can SS, Kaymak SU, Çayköylü A, Moore C, Phillips M, Ongur D. Intrinsic oscillations of auditory networks in schizophrenia and bipolar disorder. Dusunen Adam: The Journal of Psychiatry and Neurological Sciences 2019: $32(1) ; 4-13$.

Can SS, Atagün Mİ, Korkmaz ŞA, Soykan Ç. Investigating the jumping to conclusion bias in bipolar disorder. Cogn Neuropsychiatry. 2019 May;24(3):208-216. doi: 10.1080/13546805.2019.1606708.

Tunç S, Atagün Mí, Başbuğ HS, Erel Ö. Serum ceruloplasmin-ferroxidase activity in bipolar disorder is elevated compared to major depressive disorder and schizophrenia: a controlled study. Psychiatry and Clinical Psychopharmacology 2019:29 (3); 307-314.

Sahin EK, Turan G, Neselioglu S, Can SS, Atagun Mİ. Thiol-disulphide homeostasis in patients with general anxiety disorder and panic disorder. Dusunen Adam: The Journal of Psychiatry and Neurological Sciences 2019: 32; 289-294.

Altun IK, Atagün Mí. Relationship between self-esteem, emotion dysregulation and affect in borderline personality disorder. Anatolian Journal of Psychiatry. 2019; 20: 506-514.

Yusifova A, Atagün MI, Uğurlu N, Malekghasemi S, Şenat A, Demirlek C, Erel Ö. Retinal fundus imaging in bipolar disorder: A pilot study. Psychiatry Clin Neurosci. 2020;74(1):85-86. doi: 10.1111/pcn.12951.

Atagun MI, Drukker M, Hall MH, Altun İK, Tatlı SZ, Gülöksüz S, van Os J, Amelsvoort T. Meta-analysis of Auditory P50 Sensory Gating in Schizophrenia and Bipolar Disorder. Psychiatry Research: Neuroimaging. 2020; 300: 111078.

Yenilmez DO, Atagün Mí, Altun İK, Tunç S, Uzgel M, Altınbaş K, Cesur G, Oral ET. Relationship Between Childhood Adversities, Emotion Dysregulation and Cognitive Processes in Bipolar Disorder and Recurrent Depressive Disorder. Turkish Journal of Psychiatry (in press) DOI: 10.5080/u23415.

Umut G, Evren C, Atagun Mi, Hisim O, Yilmaz Çengel H, Bozkurt M, Keskinkilic C. Impact of at least 2 years of synthetic cannabinoid use on cognitive and psychomotor functions among treatment-seeking male outpatients. Cannabis and Cannabinoid Research. DOI: 10.1089/can.2019.0017. (in press) 

27 May, 2020

Maastricht, The Netherlands 